\title{
Authorized Limits for the Release of the 25-Ton Locomotive, Serial Number 21547, at the Area 25 Engine Maintenance, Assembly, and Disassembly Facility, Nevada Test Site, Nevada
}

\section{National Security Technologies, LLC Environmental Restoration Group Nevada Test Site, Nevada}

Jeremy Gwin

Douglas Frenette

May 2010 


\section{DISCLAIMER}

Reference herein to any specific commercial product, process, or service by trade name, trademark, manufacturer, or otherwise, does not necessarily constitute or imply its endorsement, recommendation, or favoring by the U.S. Government or any agency thereof. 


\section{Authorized Limits for the Release of the 25-Ton Locomotive,}

Serial Number 21547,

at the Area 25 Engine Maintenance, Assembly, and

Disassembly Facility,

Nevada Test Site, Nevada

Prepared by:

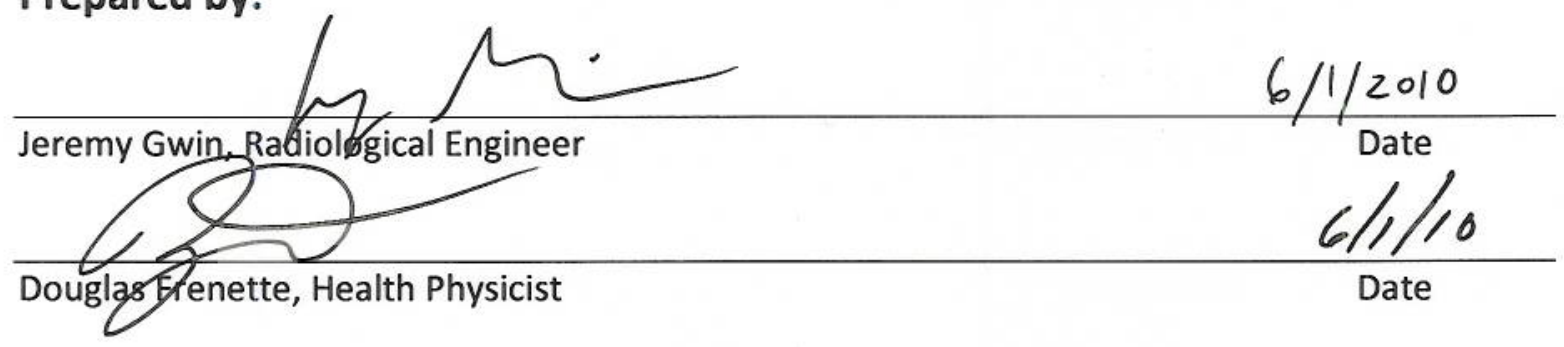

Approved by:

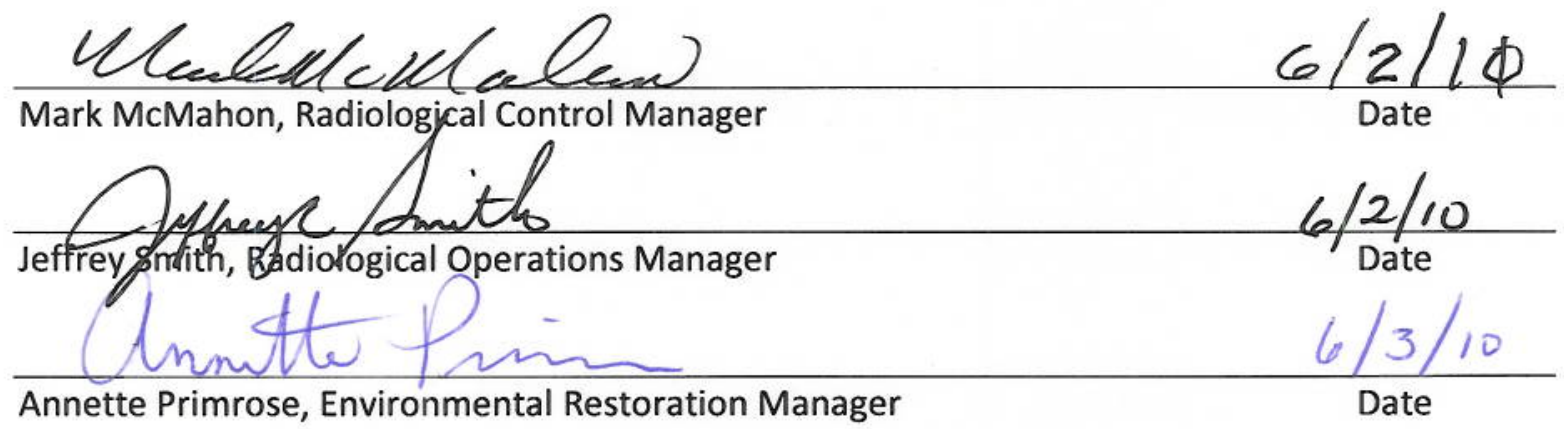




\section{PREFACE}

Records generated during the radiological assessment and clearance of material and equipment from the Nevada National Security Site (formerly known as the Nevada Test Site, or NTS) have historic value and deserve special attention. These records are maintained in accordance with National Archive and Records Administration approved U.S. Department of Energy (DOE) record schedules, as defined in National Security Technologies, LLC, procedures.

The proposed transfer of this locomotive has been discussed with the Nevada Division of Environmental Protection. DOE attended a Community Advisory Board (CAB) for NTS Programs meeting, which resulted in the $C A B$ discussing the future disposition of the locomotive to the museum. Additional public notification of the release of this locomotive will be included in the NTS Annual Environmental Report.

Independent verification of the radiological clearance of this property was performed by two U.S. Department of Energy, National Nuclear Security Administration/Nevada Site Office Subject Matter Experts, independent of the contractor responsible for the clearance action. 


\section{Table of Contents}

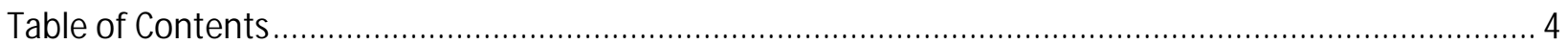

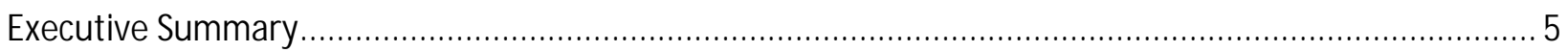

1. INTRODUCTION

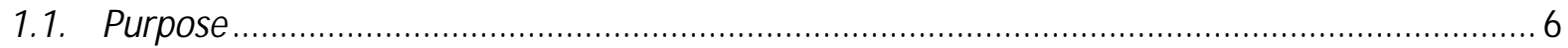

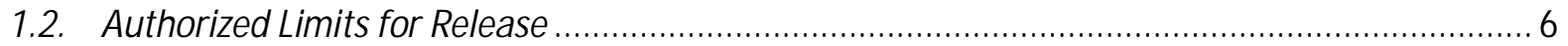

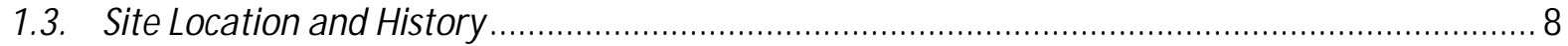

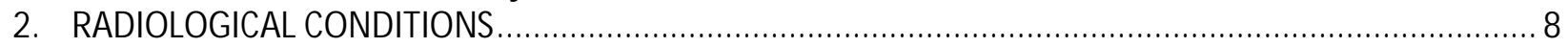

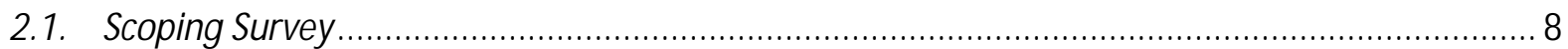

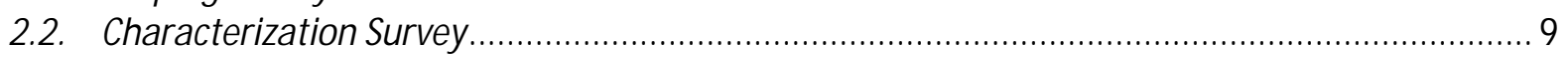

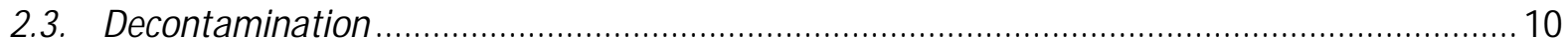

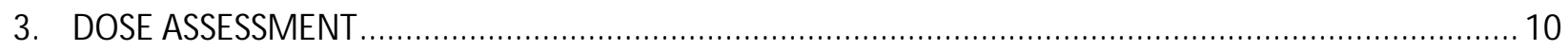

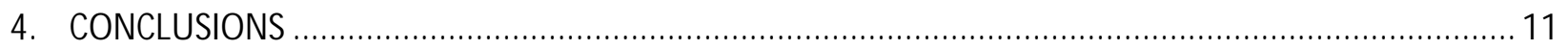

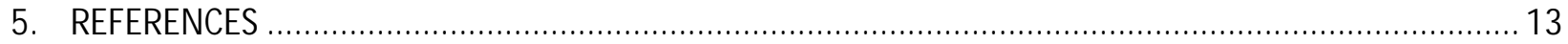

\section{List of Attachments}

ATTACHM ENT 1. Scoping Survey Results \#08-ER-A25-100

ATTACHM ENT 2. Survey Plan \#08-028, “EM AD Locomotive Survey to Determine Release from Radiological Controls Criteria"

ATTACHM ENT 3. Survey Results \#08-ER-A25-185 for Survey Plan \#08-028

ATTACHM ENT 4. Survey Plan \#09-037, "Post Remediation Survey requirements for the 25 Ton Locomotive at the Engine M aintenance, Assembly and Disassembly (EM AD) Rail Yard Serial Number 21547"

ATTACHM ENT 5. Survey Results \#10-ER-A25-097 for Survey Plan \#09-037

ATTACHM ENT 6. REC-2010-001, "Public Dose Estimate from the EM AD 25 Ton Locomotive"

\section{List of Figures}

Figure 1. EM AD 25-Ton Locomotive

\section{List of Tables}

Table 1. Table 4-2 from the NTS Radiological Control M anual .............................................................. 8

Table 2. Summary of Dose Received for Use Scenarios 


\section{Executive Summary}

This document contains the process knowledge and radiological data and analysis to support approval for offsite release of the 25-ton locomotive, Serial Number 21547, at the Area 25 Engine Maintenance, Assembly, and Disassembly (EMAD) Facility, located on the Nevada Test Site (NTS) using alternate release limits.

The 25-ton locomotive is a small, one-of-a-kind locomotive previously used to move railcars in support of the Nuclear Engine for Rocket Vehicle Application project. This locomotive was identified as having significant historical value and is now owned by the Nevada State Railroad Museum in Boulder City, Nevada. If offsite release is approved, the locomotive will be moved to the Museum where it will be used as a display piece.

As part of the process for release, a substantial effort to characterize the radiological conditions of the locomotive was undertaken by the NTS Management and Operations Contractor, National Security Technologies, LLC (NSTec). During this characterization process, seven small areas on the locomotive had contamination levels that exceeded the limits of U.S. Department of Energy [DOE] Order DOE O 5400.5, "Radiation Protection of the Public and the Environment". The decision was made to perform radiological decontamination of these known accessible impacted areas to further the release process. On February 9, 2010, NSTec personnel completed decontamination of these seven areas to levels less than the DOE 05400.5 release criteria.

Although all accessible areas of the locomotive had been successfully decontaminated to within NTS release criteria, it was plausible that inaccessible areas of the locomotive (i.e., those areas on the locomotive where it was not possible to perform radiological surveys) could potentially have contamination above unrestricted release limits. A complete radiological survey would require disassembly of the locomotive to access the majority of these inaccessible areas. Disassembly would destroy parts and would have ruined the historical value of the locomotive. Complete disassembly would also add an unreasonable financial burden for the contractor.

A decision was reached between the U.S. Department of Energy, National Nuclear Security Administration Nevada Site Office and NSTec, opting for alternative authorized limits from DOE Headquarters. In doing so, NSTec personnel performed a dose model using the DOE-approved modeling code RESRAD-BUILD v3.5 to evaluate scenarios. The parameters used in the dose model were conservative. NSTec's Radiological Engineering Calculation, REC-2010-001, "Public Dose Estimate from the EMAD 25 Ton Locomotive," concluded that the four scenarios evaluated were below the 25-millirem per year (mrem/yr) dose constraint of DOE Order 5400.5. The likely use scenarios resulted in doses less than $1 \mathrm{mrem} / \mathrm{yr}$, and the worst-case scenarios resulted in doses less than $4 \mathrm{mrem} / \mathrm{yr}$. All scenarios resulted in doses on the order of a few millirem or less in a year, thus meeting the radiological requirements for unrestricted release with residual radioactivity to the public.

Based on the worst case potential dose to a member of the public of approximately $4 \mathrm{mrem} / \mathrm{yr}$, far less than the 25- mrem/yr dose constraint of DOE Order 5400.5, NSTec is requesting the use of "a few millirem or less" criteria as the alternate authorized limit to release the 25 -ton locomotive to the public. 


\section{INTRODUCTION}

This document contains process knowledge and radiological data and analysis to support approval for the release of the 25-ton locomotive, Serial Number 21547, located at the Area 25 Engine M aintenance, Assembly, and Disassembly (EM AD) Facility on the Nevada Test Site (NTS). The 25-ton locomotive is a small, one-of-a-kind locomotive used to move railcars in support of the nuclear rocket and jet engine program known as the Nuclear Engine for Rocket Vehicle Application (NERVA) project. This locomotive was identified as having significant historical value by the Nevada State Railroad M useum in Boulder City, Nevada, where it will be used as a display piece.

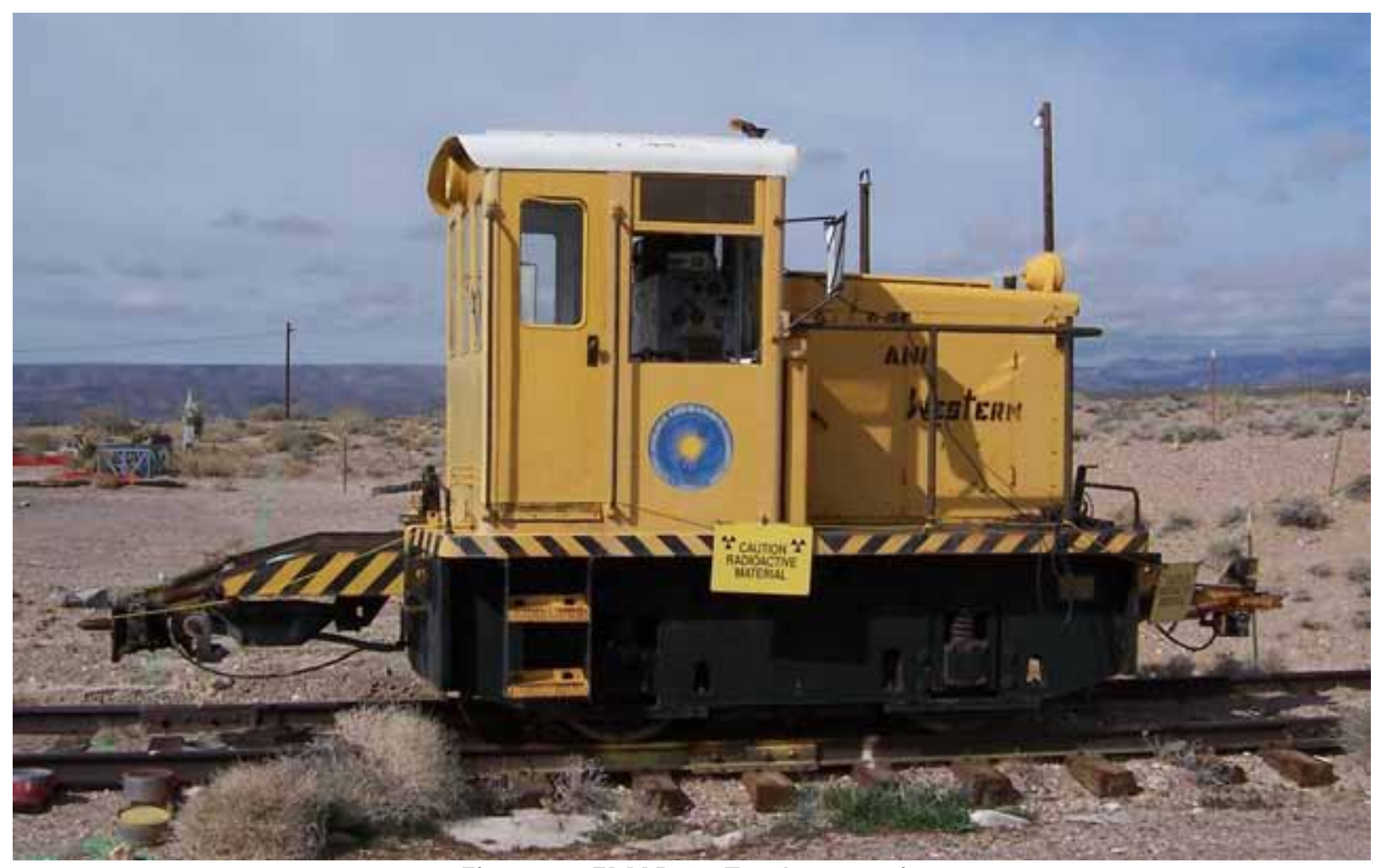

Figure 1. EMAD 25-Ton Locomotive

\subsection{Purpose}

With the radiological data and analysis presented in this document, there should be sufficient documentation for the regulator to make an informed decision for or against the approval of performing an unrestricted release of the EM AD locomotive.

\subsection{Authorized Limits for Release}

The unrestricted release of the locomotive will be based on alternate as low as reasonably achievable (ALARA)/ dose-based derived authorized limits in accordance with U.S. Department of Energy (DOE) Order DOE 0 5400.5, "Radiation Protection of the Public and the Environment." Since it is impossible to prove that there is no residual contamination in inaccessible areas, it will be necessary to demonstrate that the estimated dose to the public meets the following requirements:

- Authorized limits for release of the item must ensure that doses to the public from all sources are less than the primary dose limit for all sources (100 millirems per year [mrem/yr]). 
- Authorized limits for release of the item must be developed and approved by DOE consistent with the ALARA process. These limits will be based on a documented finding that they are as low as practicable as determined through the ALARA process, with a goal of maintaining individual doses low in comparison to background (a few mrem/yr or less). In any case, the limits must be a fraction of the primary dose limit for the public (i.e., meet a dose constraint of $25 \mathrm{mrem} / \mathrm{yr}$ or less).

DOE Guidance 441.1-XX, "Control and Release of Property with Residual Radioactive Material for use with DOE 5400.5, Radiation Protection of the Public and the Environment," recommends the following:

- The authorized limits should be selected to ensure doses to individuals using the property under "actual" and "likely use" scenarios will be well below the primary dose limit and at a level that provides a reasonable expectation doses will be less than the dose constraint of $25 \mathrm{mrem} / \mathrm{yr}$. Based on DOE experience gained in applying these requirements under DOE 5400.5, it is expected doses will be on the order of a few millirem or less in a year.

- It is not expected that the worst plausible use of property will occur, and it is not DOE's intent to permit releases expected to cause doses that are a significant fraction of the primary dose limit. In cases where the probability of the worst plausible use scenario is high and reasonably certain, the 25-mrem/yr dose constraint should be applied, ensuring doses associated with the potential release would be limited to a very small fraction of the 100-mrem/yr dose limit.

The DOE dose constraint of $25 \mathrm{mrem} / \mathrm{yr}$ is also consistent with the dose requirements in Title 10 Code of Federal Regulations (CFR) Part 20, "Standards for Protection Against Radiation," Subpart E, "Radiological Criteria for License Termination." The 25-mrem/yr dose constraint is also consistent with the Nevada Administrative Code (NAC) 459.3178, "Property of decommissioned facility: Eligibility for release for unrestricted use." A basic dose assessment using the RESRAD-BUILD v3.5 code should be adequate to demonstrate compliance with the dose constraint of $25 \mathrm{mrem} / \mathrm{yr}$.

Release of material to uncontrolled areas at the NTS is completed in accordance with the NTS Radiological Control Manual, DOE/NV/25946--801, Revision 1, Article 422, which states:

DOE O 5400.5, "Radiation Protection of the Public and the Environment," describes radiological criteria for releasing material to uncontrolled areas. Values consistent with DOE 05400.5 are provided in Table 4-2. [Tenant Organizations] must obtain NNSA/NSO approval to use any limits including those in Table 4-2 for releases to uncontrolled areas. Alternate authorized unrestricted release limits may be approved by DOE/NNSA for material, equipment, or real property with surface contamination levels greater than the Table 4-2 limits. The process to obtain approval is described in DOE 05400.5 and associated guidance documents.

Table 4-2 of the NTS Radiological Control Manual (see Table 1 below) describes surface contamination criteria for releasing material to uncontrolled areas. This table does not apply to materials that are activated or have volumetric or internal contamination. The values in Table 4-2 are the site-approved release criteria, and the limits are identical to those found in DOE O 5400.5.

According to National Security Technologies, LLC (NSTec), Organization Instruction OI-0441.212, Revision 4, "Controlled and Unrestricted Release," items that are to be released off site and have inaccessible surfaces and have known contamination or the potential for internal contamination require the approval of the Radiological Control Manager, the senior line manager of the project, and the DOE line manager. 
Table 1. Table 4-2 from the NTS Radiological Control Manual

\begin{tabular}{|c|c|c|c|}
\hline \multirow{2}{*}{ Radionuclides } & \multicolumn{3}{|c|}{ Contamination levels in $\mathrm{dpm} / 100 \mathrm{~cm}^{2}$} \\
\hline & Average & Maximum & Removable \\
\hline $\begin{array}{c}\text { Group } 1 \text { - Transuranics, I-125, I-129, Ac-227, Ra-226, Ra-228, } \\
\text { Th-228, Th-230, Pa-231 }\end{array}$ & 100 & 300 & 20 \\
\hline $\begin{array}{c}\text { Group } 2 \text { - Th-natural, Sr-90, I-126, I-131, I-133, Ra-223, Ra-224, } \\
\text { U-232, Th-232 }\end{array}$ & 1,000 & 3,000 & 200 \\
\hline $\begin{array}{c}\text { Group } 3 \text { - U-natural, U-235, U-238, and associated decay products, } \\
\text { alpha emitters }\end{array}$ & 5,000 & 15,000 & 1,000 \\
\hline $\begin{array}{l}\text { Group } 4 \text { - Beta-gamma emitters (radionuclides with decay modes } \\
\text { other than alpha emission or spontaneous fission) except Sr-90 and } \\
\text { others noted above }\end{array}$ & 5,000 & 15,000 & 1,000 \\
\hline Tritium (applicable to surface and subsurface) & N/A & $\mathrm{N} / \mathrm{A}$ & 10,000 \\
\hline
\end{tabular}

\subsection{Site Location and History}

The locomotive is currently located at the NTS, at the EM AD facility in Area 25. The locomotive was used to move railcars to support the nuclear rocket/jet engine programs. It is listed at 25 tons and has not been enhanced with shielding. It is a diesel/ electric locomotive with an electric motor mounted in the undercarriage. The locomotive utilizes a drive chain to link the motor to the drive wheels.

The locomotive was used to move flat and test cars into position and not for test articles or contaminated equipment. The low traction and poor braking made it undesirable for that type of work. However, there is photographic record of the locomotive being directly coupled to one of the test engines, and some of the rails it operated on are known to have been contaminated.

It also was used at all of the Area 25 test facilities in support of the rocket and jet engine programs. The engine was fully operational at the time that EM AD was shut down and was used to move railcars within the compound after facility closure. The main radionuclides involved in work activities consisted of residual uranium and mixed fission products. It is not known when the locomotive last operated.

\section{RADIOLOGICAL CONDITIONS}

In the past, the locomotive was posted as a Contamination Area. The reasons for these postings are not known, although a small container of radioactive material, picked up from along the railroad tracks, was temporarily stored in the cab of the locomotive. The container is no longer in the cab. The Contamination Area signs were removed, but it is not known by whom or by what process. Currently, the locomotive is posted as a Radioactive Material Area.

\subsection{Scoping Survey}

In April 2008, NSTec Radiological Control Technicians (RCTs) performed a scoping survey and identified three locations on the locomotive that had total contamination in excess of the NTS release limits, in accordance with the NTS Radiological Control Manual, DOE/NV/25946--801, Revision 1, Table 4-2. The scoping survey was documented in survey results \#08-ER-A25-100. Attachment 1 includes the complete survey results. Two of the locations were on the main deck at the front of the engine under access covers. The third location was on the front coupler. The highest direct reading was approximately 20,000 disintegrations per minute per 100 square centimeters $\left(\mathrm{dpm} / 100 \mathrm{~cm}^{2}\right)$ total beta under the access covers. The portable contamination detector used to perform the surveys was the NE Electra with a DP-6 probe. 


\subsection{Characterization Survey}

In August 2008, after development of Survey Plan \#08-028, “EM AD Locomotive Survey to Determine Release from Radiological Controls Criteria," RCTs performed an additional survey of the locomotive. Attachment 2 includes the complete survey plan. This survey was much more detailed and comprehensive than the preliminary scoping survey. The survey plan instructed RCTs to:

- Survey all exposed greased fittings, couplings, joints, etc.

- Perform swipes as indicated by photos in the survey plan (Attachment 2); biased if direct readings indicate fixed contamination present in that area.

- Survey the engine and battery compartments using a biased random technique with a minimum of 40 swipes per compartment.

- Survey the cab interior, biased random, minimum as indicated in attached photographs, focus on floor and pedals.

- Obtain a minimum of four swipes on the cab roof.

- Use large area wipes on all exposed surfaces, especially the wheels and running gear.

- Survey areas that are recessed or have small openings, such as chain drive pathways, by using a tool to push a Maslin cloth, or equivalent, into the space.

- Perform static direct surveys at all locations where swipes were taken.

- Survey for total contamination conducted over 100 percent of the accessible surface areas.

- Perform a dose rate measurement for any elevated direct reading.

Following the execution of the survey plan, the following contamination levels were noted and documented on survey \#08-ER-A25-185. Attachment 3 includes the complete survey results.

- Undercarriage Front Brake Assembly - 5,000 dpm/100 $\mathrm{cm}^{2}$ total beta

- Undercarriage Front Axle and Wheel - 7,200 dpm/100 $\mathrm{cm}^{2}$ total beta

- Front Coupler - 7,000 dpm/100 $\mathrm{cm}^{2}$ total beta

- Exterior Right Front Inspection Port - 10,800 dpm/100 $\mathrm{cm}^{2}$ total beta

- Exterior Left Front Inspection Port - $21,800 \mathrm{dpm} / 100 \mathrm{~cm}^{2}$ total beta

- Engine Compartment Right Upper Inside Lip - 11,100 dpm/100 cm² total beta

- Engine Compartment Left Upper Inside Lip - 6,800 dpm/100 $\mathrm{cm}^{2}$ total beta

Note: The radiological units " $\mathrm{dpm} / 100 \mathrm{~cm}^{2 "}$ are used when documenting surface area activity.

No removable contamination was found. The locations of the elevated fixed contamination are not immediately adjacent to any of the inaccessible areas of the locomotive. (These inaccessible areas were inaccessible for the portable survey meter.) M ost inaccessible areas were surveyed for removable contamination to some extent (swipes), except for the interior portions of the engine and other sealed components. This provided a reasonable assurance that contamination did not migrate to the inaccessible surfaces since the potential intake pathways were not contaminated and the locations with elevated contamination were remote to those pathways.

The intent of the survey plan was to evaluate the locomotive for unrestricted release. After the survey results were analyzed, the locomotive did not meet the NTS release criteria for unrestricted release.

The survey results were then used as a thorough characterization survey. With the discovery of multiple areas being impacted, the intended disposition of the locomotive to an offsite museum was postponed until a path forward for unrestricted release could be determined. 


\subsection{Decontamination}

As part of the path forward for unrestricted release, the identified areas of known fixed contamination on the locomotive were decontaminated in January-February 2010 under Survey Plan \#09-037, "Post Remediation Survey Requirements for the 25 Ton Locomotive at the Engine M aintenance, Assembly and Disassembly (EM AD) Rail Yard Serial Number 21547." Attachment 4 includes the complete survey plan. After decontamination, a post-remediation survey was performed, and the results were documented on survey \#10-ER-A25-097. Attachment 5 includes the complete survey results. In addition, the locomotive was drained of all remaining fluids.

The decontamination process effectively removed known contamination from the surfaces of the locomotive to less than detectable limits of the survey instrument, with the exception of one location. The right upper inside lip of the engine compartment was decontaminated to a beta activity of $3,000 \mathrm{dpm} / 100 \mathrm{~cm}^{2}$. The area of the fixed contamination was less than 1 square meter. It is important to note that after the decontamination was performed, the accessible areas of the locomotive met the NTS release criteria.

A sample of decontamination effluent and trash was given to NSTec Radiological Engineering to perform an isotopic identification. Performing gamma spectroscopy, the isotopes of Cs-137 and U-235 were identified. It was assumed that the beta activity is composed of a mixture of fission products (Cs-137 and $\mathrm{Sr} / \mathrm{Y}-90)$. The U-235 was identified by gamma spectroscopy but is present on the locomotive at or below the detectable limits of the portable survey instruments.

With decontamination of the accessible areas completed, there was a concern about the radiological conditions of the inaccessible areas of the locomotive. Although all accessible areas of the locomotive had been successfully decontaminated to within NTS release criteria, it was plausible that inaccessible areas of the locomotive (i.e., those areas on the locomotive where it was not possible to perform radiological surveys) could potentially have contamination above unrestricted release limits.

To access the majority of these inaccessible areas, the locomotive would have to be disassembled. Performing a complete disassembly of the locomotive would allow total access for a radiological survey but would require a substantial amount of cutting and welding on portions of the locomotive that might permanently destroy parts. These parts would have to be custom fabricated to replace, and the historical value of the locomotive would be ruined. With the possibility of destroying the historical value of the train, disassembly was not determined to be a viable option.

\section{DOSE ASSESSMENT}

With the accessible areas of the locomotive meeting the NTS release criteria, but with the unknown radiological condition of the inaccessible areas, a decision was reached between the U.S. Department of Energy, National Nuclear Security Administration Nevada Site Office and NSTec to pursue alternative authorized limits from DOE Headquarters. In order to obtain alternative authorized limits, a dose assessment would have to be performed in order to demonstrate that the dose to the public would meet regulations and be ALARA. NSTec personnel performed various dose models using the DOEapproved modeling code RESRAD-BUILD v3.5 to evaluate different likely use and worst case scenarios, as required by DOE 05400.5 .

The dose model is included in Attachment 6, Radiological Engineering Calculation REC-2010-001, Revision 1, "Public Dose Estimate from the EM AD 25 Ton Locomotive." REC-2010-001 contains the detailed descriptions of the scenarios, parameters, assumptions, and results. In short, four use 
scenarios were developed under conservative assumptions and incorporated into the dose modeling of the EM AD locomotive. The four scenarios are summarized below.

1. Likely Use Scenario 1: The dose to an individual under a likely use scenario was modeled as a member of the public, the "museum enthusiast," who attends the museum once a week for 2 hours. The entire 2 hours are spent 0.3 meters from the side of the locomotive. For conservatism, the entire side of the locomotive was assumed to be uniformly contaminated with the maximum activity that was found on previous surveys.

2. Likely Use Scenario 2: Another likely use scenario involved a "museum worker" who refurbishes or moves the locomotive for 10 hours a day for 30 days. The time was spent 0.3 meters away from the sides of the locomotive, partially surrounded by a contaminated engine compartment.

3. Worst-Case Scenario 1: A dose under worst plausible use scenarios, or "worst-case" scenario, would represent a situation if all controls failed. A scenario was hypothesized and involved an individual spending an unreasonable amount of time in close proximity to a contaminated engine compartment. This person would spend 8 hours a day, every day, at an average distance of 0.3 meters from the side of the "contaminated" compartment.

4. Worst-Case Scenario 2: Another worst-case scenario involved a museum employee who mechanically removes (by grinding or sanding) the small area of fixed contamination that remains on the locomotive. This scenario assumed that the total amount of known contamination becomes airborne, that the employee is 0.3 meters away, and spends 6 hours performing work.

The RESRAD-BUILD detailed results for each scenario are included in Appendices B-E of Attachment 6. A summary of the doses received for each scenario over time is presented in Table 2 . The Radiological Engineering Calculation concluded that all four scenarios evaluated were below the $25-\mathrm{mrem} / \mathrm{yr}$ limit and met the "few millirem in a year" criteria.

Table 2. Summary of Dose Received for Use Scenarios

\begin{tabular}{|c|c|c|c|c||}
\hline \multirow{2}{*}{ Scenario } & \multicolumn{3}{|c|}{ Annual Dose Received for the Maximum Exposed Individual (mrem) } \\
\hline Likely Use 1 & $\begin{array}{c}\text { End of the } \mathbf{1}^{\text {st }} \\
\text { year }\end{array}$ & $\begin{array}{c}\text { End of the } \mathbf{2}^{\text {nd }} \\
\text { year }\end{array}$ & $\begin{array}{c}\text { End of the } \mathbf{1 0}^{\text {th }} \\
\text { year }\end{array}$ & $\begin{array}{c}\text { End of the } \mathbf{3 0}^{\text {th }} \\
\text { year }\end{array}$ \\
\hline Likely Use 2 & 0.109 & 0.105 & 0.085 & 0.0536 \\
\hline Worst Case 1 & 3.512 & 0.491 & 0.399 & 0.252 \\
\hline Worst Case 2 & 1.16 & 3.30 & 2.68 & 1.69 \\
\hline \hline
\end{tabular}

\section{CONCLUSIONS}

The Nevada State Railroad M useum in Boulder City, Nevada, intends to use the locomotive as a public display only. This locomotive will not be operational; it will just be used as a static display for the public to view. Current rules at the rail yard prohibit the public from climbing on any of the displays. The rail yard has a fence around the train displays and is locked after business hours. The only foreseeable direct contact with the locomotive is from museum workers if they have to move it or refurbish it.

The scenarios modeled in RESRAD-BUILD v3.5 are very conservative. The conservative assumptions, including the exaggerated source term and the amount of time spent in close contact with the 
locomotive, have a high probability of overestimating the dose to the individual. Even with these conservative assumptions, the maximum dose to an individual for the likely use scenarios was less than $1 \mathrm{mrem} / \mathrm{yr}$, and the maximum dose for the worst-case scenarios was less than $4 \mathrm{mrem} / \mathrm{yr}$.

The "Likely Use Scenario 1" represents members of the public visiting the museum and viewing the locomotive. The "museum enthusiast" could receive a maximum dose of $0.109 \mathrm{mrem} / \mathrm{yr}$. This scenario has the highest probability of occurring out of the four scenarios. This individual would spend 2 hours per week, every week for 30 years, standing 0.3 meters from the exterior surface of the locomotive. The expected dose to an individual in this group would be a fraction of the calculated dose because the source term was exaggerated in the RESRAD-BUILD code. The calculated maximum dose under this likely use scenario is well under the $25-\mathrm{mrem} / \mathrm{yr}$ dose constraint and meets the criteria of a "few millirem or less in a year" established in DOE 0 5400.5.

The "Likely Use Scenario 2" represents employees of the museum who refurbish or move the locomotive. The "museum worker" could receive a maximum dose of $0.512 \mathrm{mrem} / \mathrm{yr}$. This scenario is less probable than "Likely Use Scenario 1." The museum employee would spend 10 hours a day, for 30 days a year, positioned partially enclosed between three contaminated surfaces at 0.3 meters from each surface. The expected dose to an individual in this group would be a fraction of the calculated dose because the source term was exaggerated in the RESRAD-BUILD code. The calculated maximum dose under the likely use scenario is well under the $25-\mathrm{mrem} / \mathrm{yr}$ dose constraint and meets the criteria of a "few millirem or less in a year" established in DOE 05400.5.

Under the "Worst-Case Scenario 1," an individual could receive a maximum dose of $3.83 \mathrm{mrem} / \mathrm{yr}$. The worst-case scenario represented the dose to an individual if all controls failed. This individual would spend 8 hours per day, every day for 30 years, in close proximity to a surface contaminated engine compartment. The shielding thickness was decreased, and the source activity for the "contaminated" engine compartment was exaggerated, thus increasing the dose to the individual. The probability of this scenario occurring is low. The calculated maximum dose under the worst-case scenario is well under the 25-mrem/yr dose constraint established in DOE 0 5400.5.

Under the "Worst-Case Scenario 2," an individual could receive a maximum dose of $1.16 \mathrm{mrem} / \mathrm{yr}$. The individual would receive a one-time 1.16-mrem dose during a 6 hour exposure. This individual would spend a total of 6 hours mechanically removing a small surface contaminated area. The source activity for the contaminated area mimicked actual conditions. The airborne respirable fraction was set at 50 percent, which equates to half of the total source activity becoming airborne and existing as respirable particulate. This was a conservative assumption because most of the source activity would not exist as respirable fractions during mechanical removal (i.e., mechanical removal fragments would be too large to become respirable). The probability of this scenario occurring is low. The calculated maximum dose under this scenario is well under the $25-\mathrm{mrem} / \mathrm{yr}$ dose constraint established in DOE 05400.5.

With all four scenarios less than the 25-mrem/yr limit and with the likely dose scenarios meeting the "few millirem in a year" criteria, the EM AD 25-ton locomotive, as it currently exists, meets the radiological requirements of DOE 05400.5 to have an unrestricted release to the public. NSTec is requesting the use of "a few millirem or less" criteria as the alternate authorized limit to release the 25-ton locomotive to the public. 


\section{REFERENCES}

Code of Federal Regulations, 2008. Title 10, Part 835, "Occupational Radiation Protection." Washington, D.C.

Code of Federal Regulations, 1998. Title 10, Part 20, "Standard for Protection Against Radiation." Washington, D.C.

U.S. Department of Energy, 1993. DOE O 5400.5, "Radiation Protection of the Public and the Environment." Washington, D.C.

U.S. Department of Energy, 1999. DOE Guidance 441.1-XX, "Control and Release of Property with Residual Radioactive M aterial for use with DOE 5400.5, Radiation Protection of the Public and the Environment." Washington, D.C.

U.S. Department of Energy, National Nuclear Security Administration Nevada Site Office, 2010. Nevada Test Site Radiological Control Manual, Revision 1. DOE/NV/25946--801. Las Vegas, NV.

National Security Technologies, LLC, 2008. Company Document CD-0441.004, “M aterial Release Program." Las Vegas, NV.

National Security Technologies, LLC, 2008. Organization Instruction OI-0441.212, “Controlled and Unrestricted Release," Revision 4. Las Vegas, NV. 
Authorized Limits for Release of the

EM AD 25-Ton Locomotive

May 2010

\section{ATTACHMENT 1 \\ Scoping Survey Results \#08-ER-A25-100}


NSTec

RADIOLOGICAL SURVEY

REPORT - DATA

\begin{tabular}{|c|c|c|c|c|c|c|c|c|c|c|c|}
\hline \multicolumn{2}{|c|}{$\begin{array}{l}\text { Location: } \\
\text { Area 25, E-MAD }\end{array}$} & \multicolumn{3}{|c|}{$\begin{array}{l}\text { Purpose: } \\
\text { Cursory survey of train engine to be } \\
\text { transferred off site to a museum }\end{array}$} & \multicolumn{5}{|c|}{$\begin{array}{l}\text { Comments: } \\
\text { All Swipes Field Checked Highest Readings Noted. } \\
\text { Train is within a posted controlled area and RMA }\end{array}$} & \multicolumn{2}{|c|}{$\begin{array}{l}\text { Date/Time: } \\
3 / 12 / 08 \text { / } 1330\end{array}$} \\
\hline Instrument: & Serial \#: & $\frac{1}{\text { Cal Due: }}$ & $\begin{array}{c}\text { Eff in } \%: \\
\text { Alpha / Beta }\end{array}$ & $\begin{array}{l}\text { BKG in dpm: } \\
\text { Alpha } / \text { Beta }\end{array}$ & \multicolumn{2}{|c|}{$\begin{array}{l}\text { MDA in dpm: } \\
\text { Alpha } / \quad \text { Beta }\end{array}$} & \multicolumn{5}{|c|}{ RWP \# N / A } \\
\hline 2929 & 157336 & $12 / 31 / 08$ & $35.39 / 42.03$ & $0.85 / 224.8$ & \multicolumn{2}{|c|}{$13.0 / 86.5$} & \multicolumn{5}{|c|}{ Work Package \# N/A } \\
\hline Electra & 2224 & $9 / 13 / 08$ & $15.2 / 24.1$ & $32.9 / 2828$ & \multicolumn{2}{|c|}{$80 / 500$} & \multicolumn{2}{|c|}{ RCT Name: S. Munns } & \multicolumn{3}{|c|}{ Signature: $/ /$ tees } \\
\hline Model-3 & 197130 & $4 / 26 / 08$ & $\mathrm{~N} / \mathrm{A}$ & $\mathrm{N} / \mathrm{A}$ & \multicolumn{2}{|c|}{$\mathrm{N} / \mathrm{A}$} & \multicolumn{2}{|c|}{ RCT Name; N/A } & \multicolumn{3}{|c|}{ Signature: N/A } \\
\hline N/A & $N / A$ & $\mathrm{~N} / \mathrm{A}$ & $\mathrm{N} / \mathrm{A}$ & $\mathrm{N} / \mathrm{A}$ & \multicolumn{2}{|c|}{$N / A$} & \multicolumn{2}{|c|}{ RCT Name; N/A } & \multicolumn{3}{|c|}{ Signature: N/A } \\
\hline $\mathbf{N} / \mathbf{A}$ & $N / A$ & $\mathrm{~N} / \mathrm{A}$ & $\mathrm{N} / \mathrm{A}$ & $\mathrm{N} / \mathrm{A}$ & \multicolumn{2}{|c|}{$\mathrm{N} / \mathrm{A}$} & \multicolumn{2}{|c|}{ RCT Name: N/A } & \multicolumn{3}{|c|}{ Signature: N/A } \\
\hline \multirow{2}{*}{$\begin{array}{l}\text { Survey } \\
\text { Point }\end{array}$} & & Descriotion & mments & & & & $\begin{array}{r}\text { Fixed }+ \\
\text { dpm }\end{array}$ & $\begin{array}{l}\text { movable } \\
0 \mathrm{em}^{2}\end{array}$ & Gamma & Neutron & Total \\
\hline & & & & & Alpha & Beta & Alpha & Beta & & $\mathrm{mrem} / \mathrm{hr}$ & \\
\hline 1 & & Front couple & echanism & & $<\mathrm{MDA}$ & $<\mathrm{MDA}$ & $<\mathrm{MDA}$ & 8000 & $\mathrm{~N} / \mathrm{A}$ & $N / A$ & $N / A$ \\
\hline 2 & $\operatorname{Rec}$ & gular inspectic & ort left front de & & $<\mathrm{MDA}$ & $<\mathrm{MDA}$ & $\angle \mathrm{MDA}$ & 20,000 & $\mathrm{~N} / \mathrm{A}$ & $\mathrm{N} / \mathrm{A}$ & $N / A$ \\
\hline 3 & Rect & ular inspectio & ort right front $d$ & & $\angle \mathrm{MDA}$ & $<\mathrm{MDA}$ & $\angle \mathrm{MDA}$ & 10,000 & $\mathrm{~N} / \mathrm{A}$ & $\mathrm{N} / \mathrm{A}$ & $\mathrm{N} / \mathrm{A}$ \\
\hline 4 & & Sample F & Area & & $\angle \mathrm{MDA}$ & $\angle \mathrm{MDA}$ & $\mathrm{N} / \mathrm{A}$ & $N / A$ & $N / A$ & $N / A$ & $N / A$ \\
\hline$N / A$ & No other cont & nation found & is time; but the & are a vast & $\mathrm{N} / \mathrm{A}$ & $\mathrm{N} / \mathrm{A}$ & $N / A$ & $\mathrm{~N} / \mathrm{A}$ & $\mathrm{N} / \mathrm{A}$ & $N / A$ & $\mathrm{~N} / \mathrm{A}$ \\
\hline$N / A$ & majorit & areas of the & that are inacces & ble. & $\mathrm{N} / \mathrm{A}$ & $\mathrm{N} / \mathrm{A}$ & $\mathrm{N} / \mathrm{A}$ & $\mathrm{N} / \mathrm{A}$ & $\mathrm{N} / \mathrm{A}$ & $N / A$ & $N / A$ \\
\hline $\mathrm{N} / \mathrm{A}$ & Also once these & ee areas were & ind the survey & s terminated & $\mathrm{N} / \mathrm{A}$ & $N / A$ & $\mathrm{~N} / \mathrm{A}$ & N/A & $\mathrm{N} / \mathrm{A}$ & $N / A$ & $N / A$ \\
\hline$N / A$ & due to the $\mathrm{r}$ & n for perform & the survey to $b$ & in with. & $\mathrm{N} / \mathrm{A}$ & $N / A$ & $N / A$ & $\mathrm{~N} / \mathrm{A}$ & $\mathrm{N} / \mathrm{A}$ & $\mathrm{N} / \mathrm{A}$ & $\mathrm{N} / \mathrm{A}$ \\
\hline $\mathrm{N} / \mathrm{A}$ & & $\mathrm{N}$ & & & $\mathrm{N} / \mathrm{A}$ & $\mathrm{N} / \mathrm{A}$ & $\mathrm{N} / \mathrm{A}$ & $N / A$ & N/A & $\mathrm{N} / \mathrm{A}$ & $N / A$ \\
\hline $\mathrm{N} / \mathrm{A}$ & & $\mathrm{N}$ & & & $\mathrm{N} / \mathrm{A}$ & $N / A$ & $\mathrm{~N} / \mathrm{A}$ & $\mathrm{N} / \mathrm{A}$ & $\mathrm{N} / \mathrm{A}$ & $\mathrm{N} / \mathrm{A}$ & $N / A$ \\
\hline $\mathrm{N} / \mathrm{A}$ & & $\mathrm{N}$ & & & $\mathrm{N} / \mathrm{A}$ & $\mathrm{N} / \mathrm{A}$ & $\mathrm{N} / \mathrm{A}$ & $\mathrm{N} / \mathrm{A}$ & $\mathrm{N} / \mathrm{A}$ & $\mathrm{N} / \mathrm{A}$ & $\mathrm{N} / \mathrm{A}$ \\
\hline$N / A$ & & $\mathrm{~N}$ & & & $\mathrm{~N} / \mathrm{A}$ & $N / A$ & $N / A$ & $\mathrm{~N} / \mathrm{A}$ & $\mathrm{N} / \mathrm{A}$ & $\mathrm{N} / \mathrm{A}$ & $\mathrm{N} / \mathrm{A}$ \\
\hline $\mathrm{N} / \mathrm{A}$ & & $\mathrm{N}$ & & & $\mathrm{N} / \mathrm{A}$ & $\mathrm{N} / \mathrm{A}$ & $\mathrm{N} / \mathrm{A}$ & $\mathrm{N} / \mathrm{A}$ & $\mathrm{N} / \mathrm{A}$ & $N / A$ & $N / A$ \\
\hline $\mathrm{N} / \mathrm{A}$ & & $\mathrm{N}$ & & & $\mathrm{N} / \mathrm{A}$ & $\mathrm{N} / \mathrm{A}$ & $\mathrm{N} / \mathrm{A}$ & $\mathrm{N} / \mathrm{A}$ & $\mathrm{N} / \mathrm{A}$ & $\mathrm{N} / \mathrm{A}$ & $\mathrm{N} / \mathrm{A}$ \\
\hline $\mathrm{N} / \mathrm{A}$ & & $\mathrm{N}$ & & & $\mathrm{N} / \mathrm{A}$ & $N / A$ & $\mathrm{~N} / \mathrm{A}$ & $N / A$ & $\mathrm{~N} / \mathrm{A}$ & $\mathrm{N} / \mathrm{A}$ & $\mathrm{N} / \mathrm{A}$ \\
\hline Reviewed By & & & & Signature: & & & & & & . & \\
\hline
\end{tabular}

Page 1 of 1

SURVEY \# 08-ER-A25-100

Daterime:

All Swipes Field Checked Highest Readings Noted.

Train is within a posted controlled area and RMA 
Authorized Limits for Release of the

EM AD 25-Ton Locomotive

May 2010

ATTACHMENT 2

Survey Plan \#08-028, “EMAD Locomotive Survey to Determine Release from Radiological Controls Criteria" 


\section{Radiological Survey Plan \\ for Environmental Restoration}

\section{Engine Maintenance, Assembly, and Disassembly (EMAD) Locomotive Survey to Determine Release from Radiological Controls Criteria}

Plan Number 08-028

July 22,2008

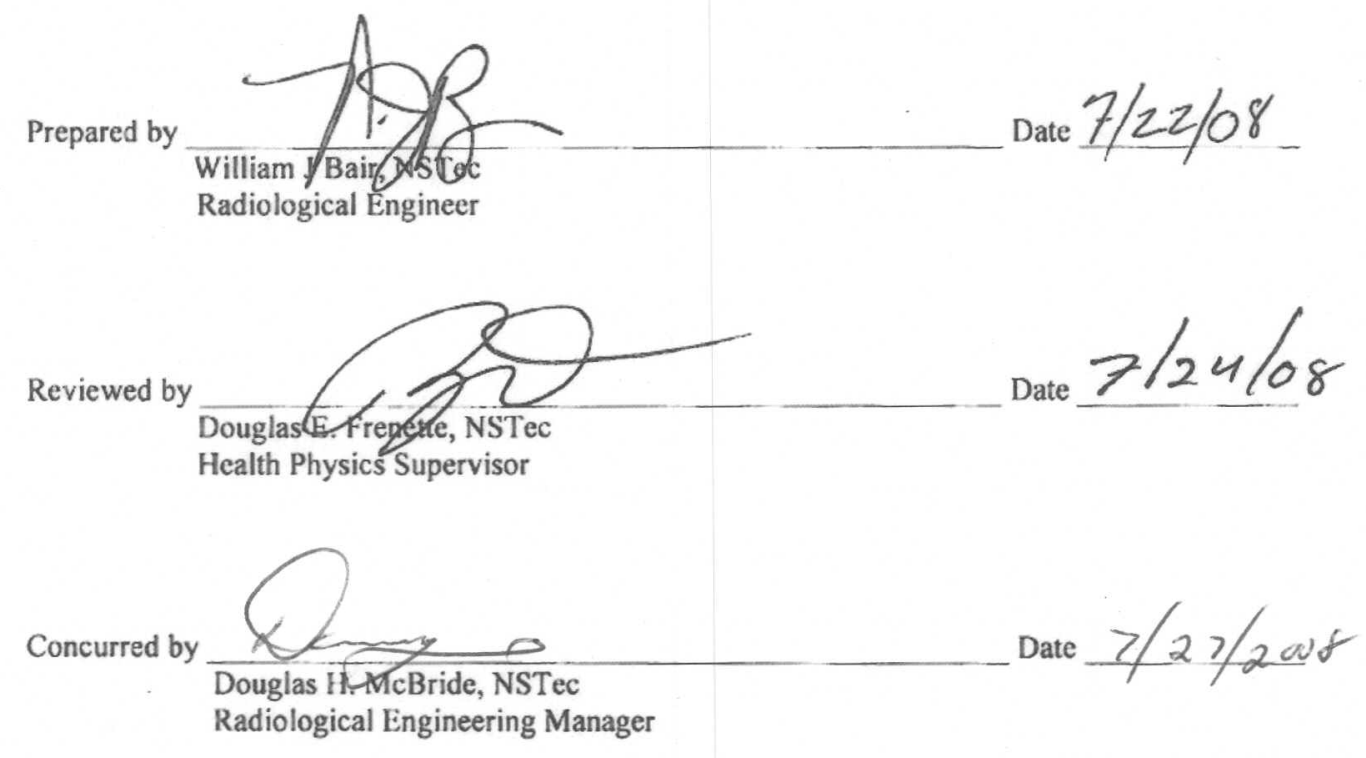

Concurred by

Approved by

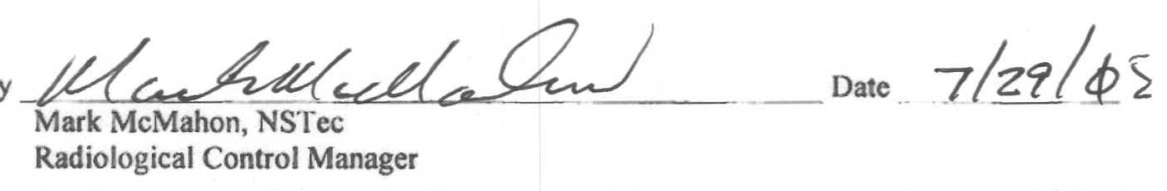




\section{Engine Maintenance, Assembly, and Disassembly (EMAD) \\ Locomotive Survey to Determine Release from Radiological Controls Criteria}

\section{Purpose}

This plan provides Radiological Control Technicians (RCTs) with the survey instructions required to determine whether the small rail locomotive, serial number 21547 may be provided a restricted release to the Nevada State Railroad Museum in Boulder City, Nevada under U.S. Department of Energy Order DOE O 5400.5, "Radiation Protection of the Public and the Environment."

\section{Scope}

This plan pertains to the small rail locomotive, serial number 21547, currently located in the EMAD yard, just north of the facility. Results of this survey will be used to determine appropriate release from radiological control levels. These levels will be evaluated with consideration for the proposed use restrictions and deemed ALARA prior to relinquishing site control.

The methodology utilized in the development of this plan was obtained from DOE 5400.5, "Radiation Protection of the Public and the Environment," DOE G 441.1-XX, "Implementation Guide for Control and Release of Property with Residual Radioactive Material,” DOE-XXXX, “Environmental Implementation Guide for Radiological Survey Procedures,” February 1997, and DOE/NV/11718-079-Rev 5, "NV/YMP Radiological Control Manual.”

\section{History}

The small locomotive was used to move railcars in support of the nuclear rocket/jet engine programs. It is listed at 25 tons and has not been enhanced with added shielding. It is a diesel/electric locomotive with an electric motor mounted in the undercarriage. The locomotive utilizes a drive chain to link the motor to the drive wheels. Testimony states that it was used to move flat and test cars into position and not for test articles or contaminated equipment. The low traction and poor braking made it undesirable for that type of work. However, there is photographic record of the locomotive being directly coupled to one of the test engines and some of the rails it operated on are known to have been contaminated. It also was used at all of the Area 25 test facilities in support of the rocket and jet engine programs. The engine was fully operational at the time that EMAD was shut down and was used to move railcars within the compound after facility closure. It is not known when it last operated. Recently, it was posted as a contamination area and as a radioactive material area. The reasons for these postings are not really known, although there is testimony that a small container of radioactive material, picked up from along the railroad tracks, was temporarily stored in the cab of the locomotive. The container is no longer in the cab and to the best knowledge available no longer on the EMAD Site. The contamination area signs were removed but it is not known by whom or by what process. RCTs performing a casual survey identified three locations on the locomotive that had direct reading contamination in excess of the release limits. Two of the locations were on the main deck at the front of the engine under access covers. The third location was on the front coupler. The highest direct reading was $\sim 20,000 \mathrm{dpm} / 100 \mathrm{~cm}^{2}$ beta under the access 


\section{Engine Maintenance, Assembly, and Disassembly (EMAD) Locomotive Survey to Determine Release from Radiological Controls Criteria}

covers. No further surveys were conducted at that time and the levels have not been verified. Previous surveys of the locomotive have not identified contamination above posting criteria of Table 2-2 of the Radiological Control Manual.

Contamination characterization for this equipment is based on IT 2000, "Cesium 137 Scaling Factors for Concrete, Insulation, Wood, and Concrete Surfaces for the Former ROVER Project and NERVA Facilities in Area 25 Nevada Test Site, Nevada.” This document shows Cs ${ }^{137}$ and enriched uranium (EU) to be the predominant contaminants of concern; $\mathrm{Sr}^{90}$ appears in equilibrium with $\mathrm{Cs}^{137}$.

\section{Instructions}

Radiological surveys shall be performed by qualified personnel in compliance with OI0441.211, "Direct and Indirect Surveys.” Personnel must use their experience, knowledge of the area in which they are working, and workplace indicators to assure adequate surveys are being performed, in addition to the information provided in this document.

The batteries and fluids will be removed from the locomotive prior to release from the site. The batteries will need to be removed prior to completion of the survey. Some locations, such as the wood floor in the cab, are not ideally suited for survey. However, since one of the dominant contaminants of concern is $\mathrm{Cs}^{137}$ the porosity of the wood should not greatly hinder detection of contamination if present above the release criteria. Dose rate surveys will also verify whether there is an issue with these surfaces regarding release of the locomotive from radiological control.

Minimum survey requirements are also found in Organizational Instruction OI-0441.212, "Controlled and Uncontrolled Release." This document is not intended to conflict with the aforementioned OI, but to provide additional guidance. This procedure also contains the specifications of the instruments' detection capabilities.

All access doors shall be opened to facilitate survey and moving parts shall be articulated or rotated to expose concealed elements. If background radiation levels are too high to allow direct measurements and dose rate surveys to be performed, then the locomotive shall be relocated to an area suitable for survey.

\section{Contamination Surveys}

The following survey methodology is consistent with the requirements of DOE-XXXX, "Environmental Implementation Guide for Radiological Survey Procedures,” February 1997. The survey points selected were based on the one per square meter recommendation of the referenced guidance document and then biased, with additional points added, to increase the probability of locating contaminated areas. Random surveys shall be conducted on the equipment for removable contamination. White dots on the attached pictures indicate the locations for swipe surveys to be taken. These are the minimum required and the RCT is encouraged to take more if an area looks suspicious or if direct readings indicate a potential exists. Surveys for removable contamination shall be performed on: 


\section{Engine Maintenance, Assembly, and Disassembly (EMAD) Locomotive Survey to Determine Release from Radiological Controls Criteria}

- All exposed greased fittings, couplings, joints, etc.

- Swipes at a minimum as indicated on the attached pictures, biased if direct readings indicate fixed contamination present in that area

- The engine and battery compartments using biased random technique; minimum of 40 swipes per compartment

- The cab interior, biased random, minimum as indicated in attached photographs, focus on floor and pedals

- Minimum of four swipes on the cab roof

- Large area wipes shall be taken on all exposed surfaces, especially the wheels and running gear

- Areas that are recessed or have small openings, such as chain drive pathways, shall be surveyed using a tool to push a Maslin cloth or equivalent into the space

- Static direct surveys shall be performed at all locations where swipes are taken

Surveys for total contamination shall be conducted over $100 \%$ of the accessible surface areas to the extent possible using an Electra survey meter. Swipes shall be counted in a Tennelec or other approved swipe counter. Locations with elevated direct readings shall be surveyed using a micro rem meter and a general dose rate of the locomotive shall be recorded. The rigor of this survey plan provides assurance that any inaccessible areas not surveyed are either identified or are below the release limits of Table 4-2.

\section{Disposition}

Survey results shall be recorded on a FRM-0108, "Radiological Survey Report," and approved by Project Health Physics Supervisor. Final approval for the release of this locomotive will be made by NNSA based on the projected dose consequence and use controls to be implemented when released for DOE control. 
Engine Maintenance, Assembly, and Disassembly (EMAD) Locomotive Survey to Determine Release from Radiological Controls Criteria
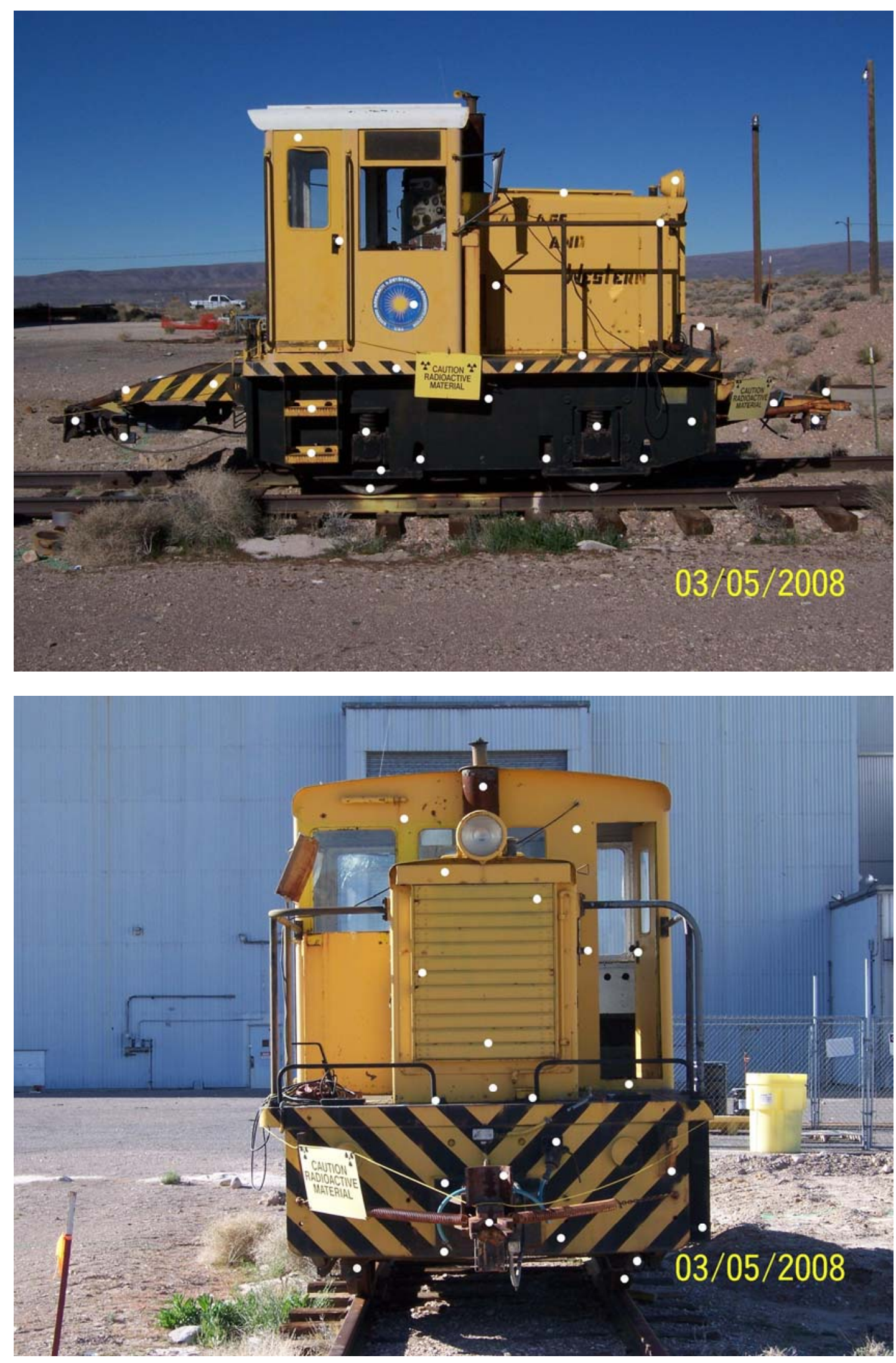
Engine Maintenance, Assembly, and Disassembly (EMAD) Locomotive Survey to Determine Release from Radiological Controls Criteria
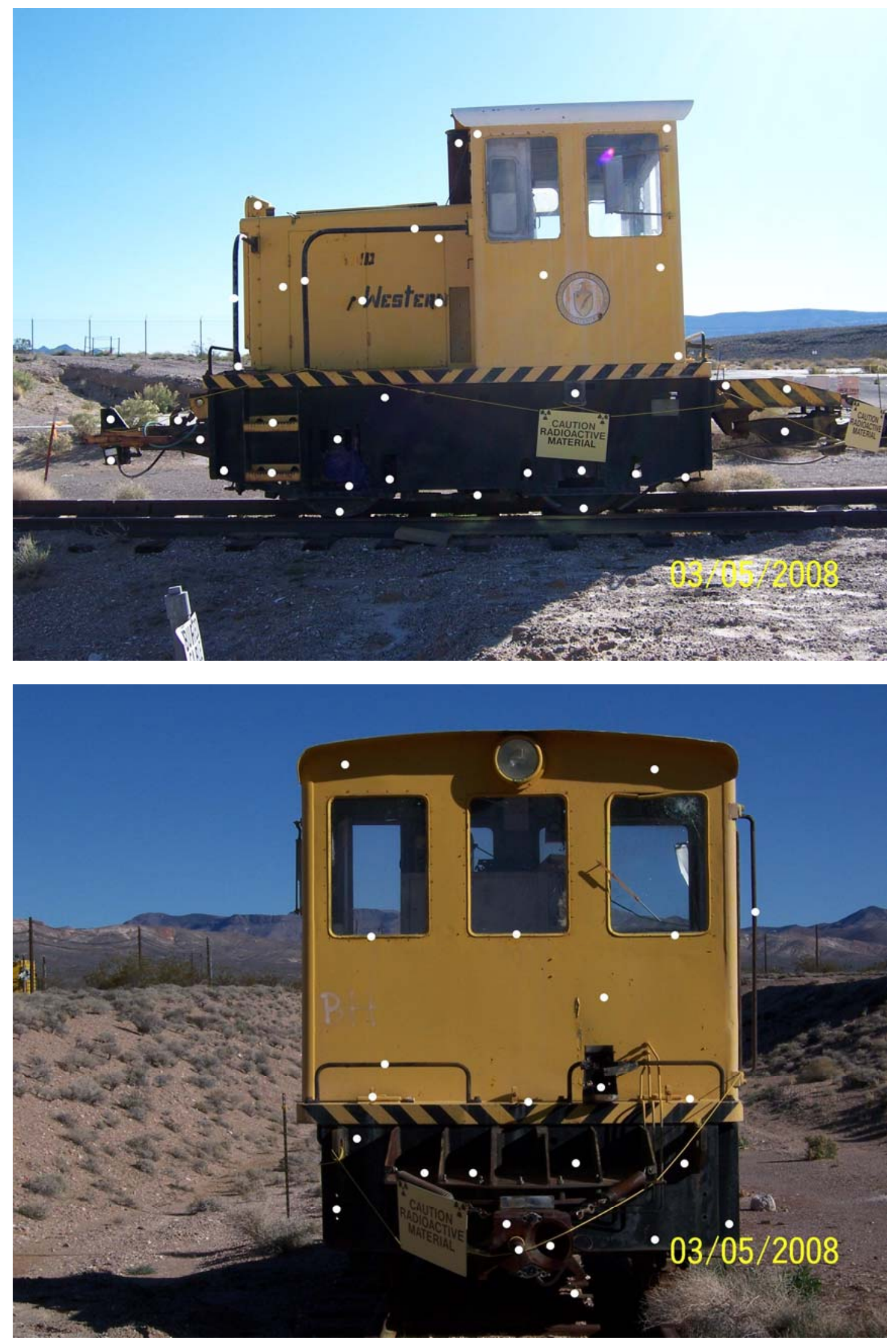
Engine Maintenance, Assembly, and Disassembly (EMAD) Locomotive Survey to Determine Release from Radiological Controls Criteria
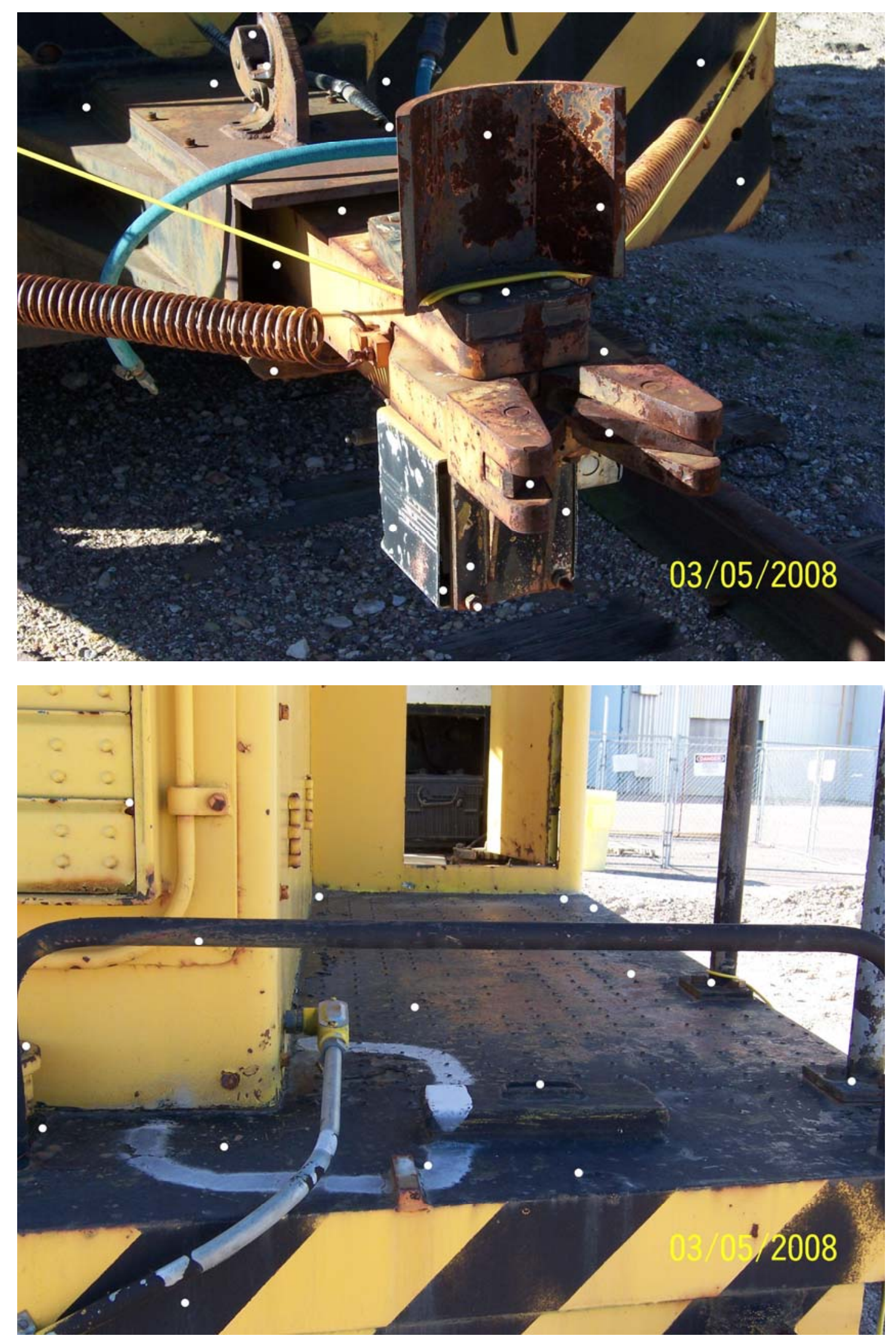
Engine Maintenance, Assembly, and Disassembly (EMAD) Locomotive Survey to Determine Release from Radiological Controls Criteria
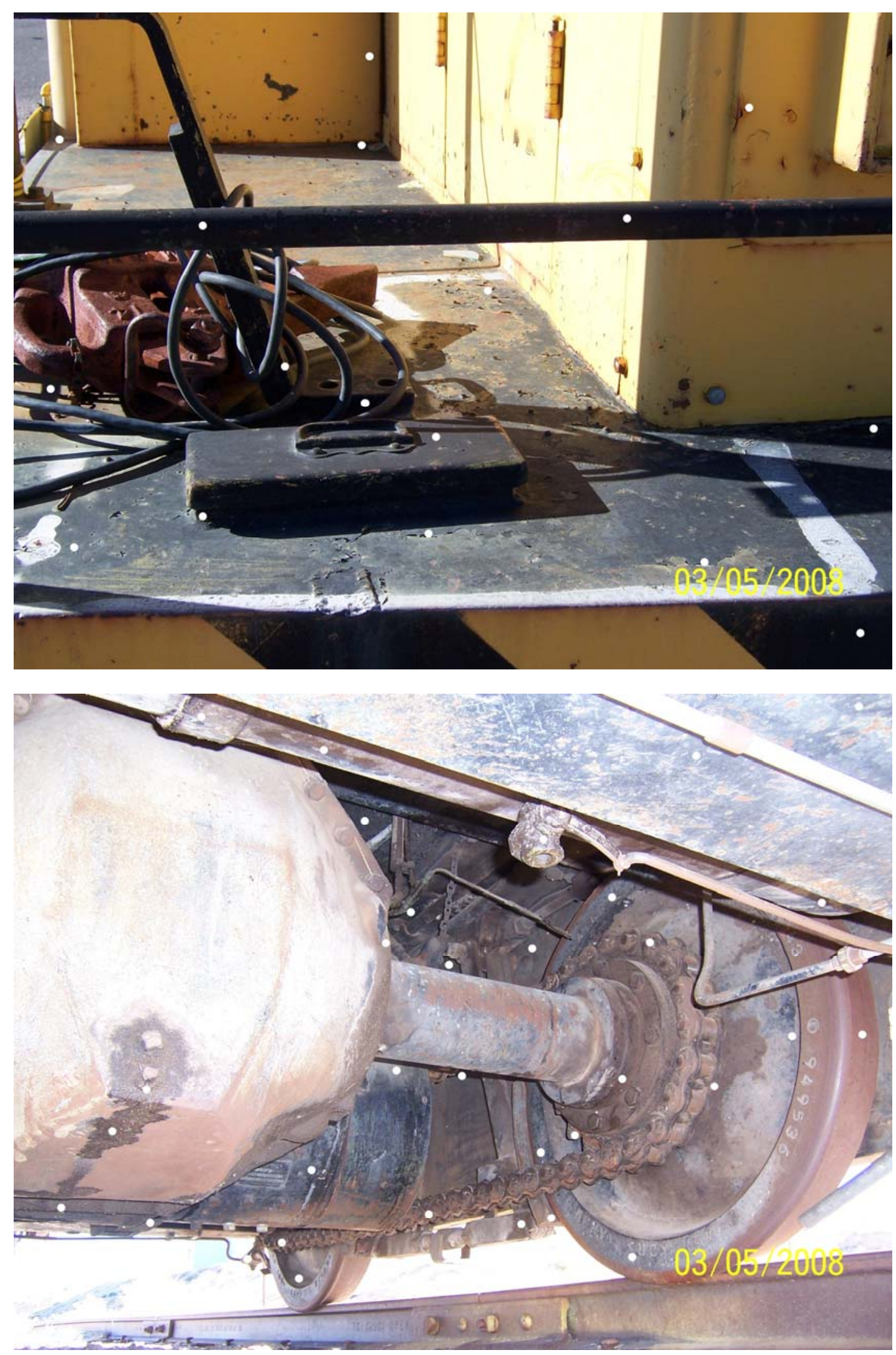
Engine Maintenance, Assembly, and Disassembly (EMAD) Locomotive Survey to Determine Release from Radiological Controls Criteria
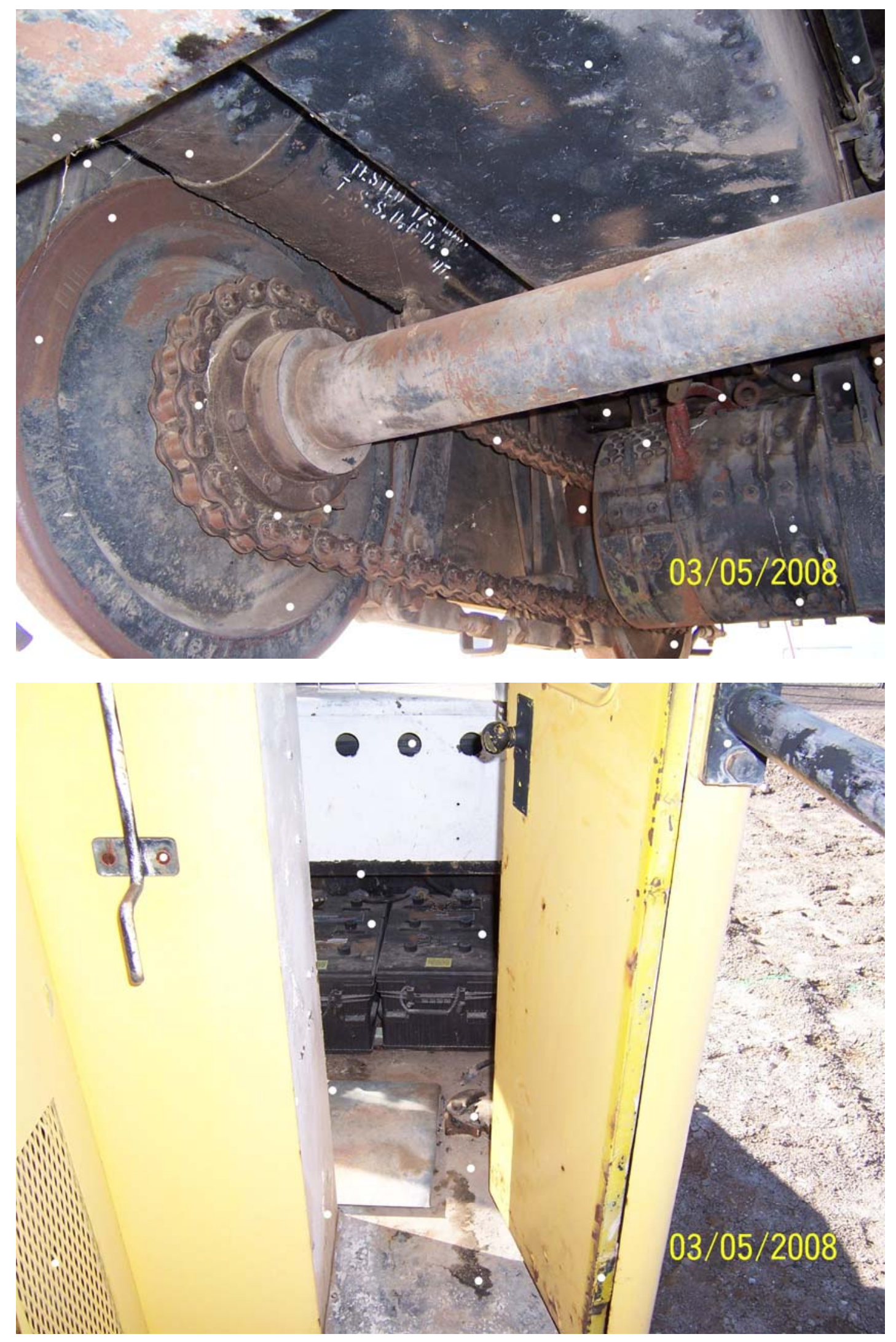
Engine Maintenance, Assembly, and Disassembly (EMAD) Locomotive Survey to Determine Release from Radiological Controls Criteria
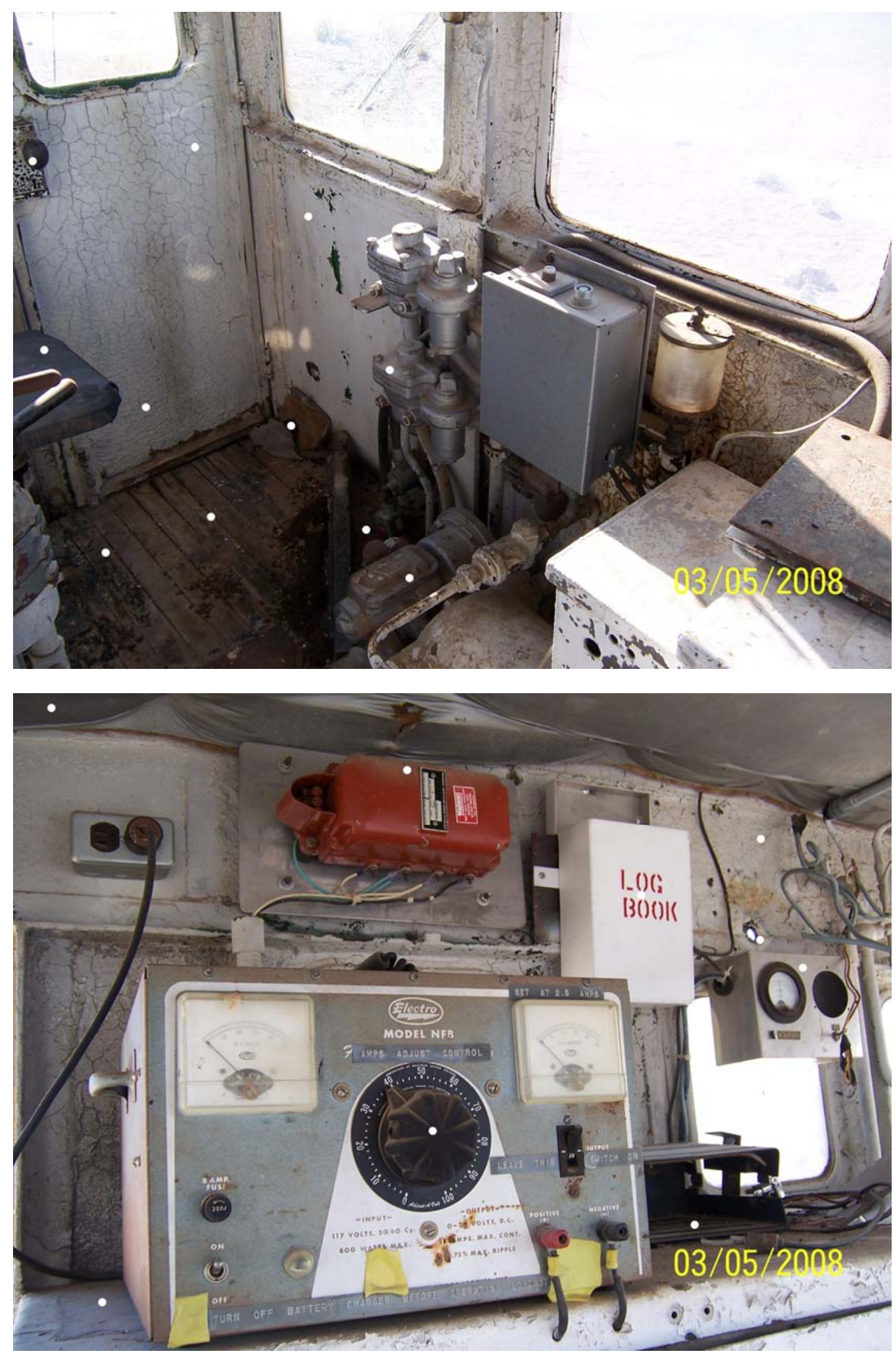
Engine Maintenance, Assembly, and Disassembly (EMAD) Locomotive Survey to Determine Release from Radiological Controls Criteria
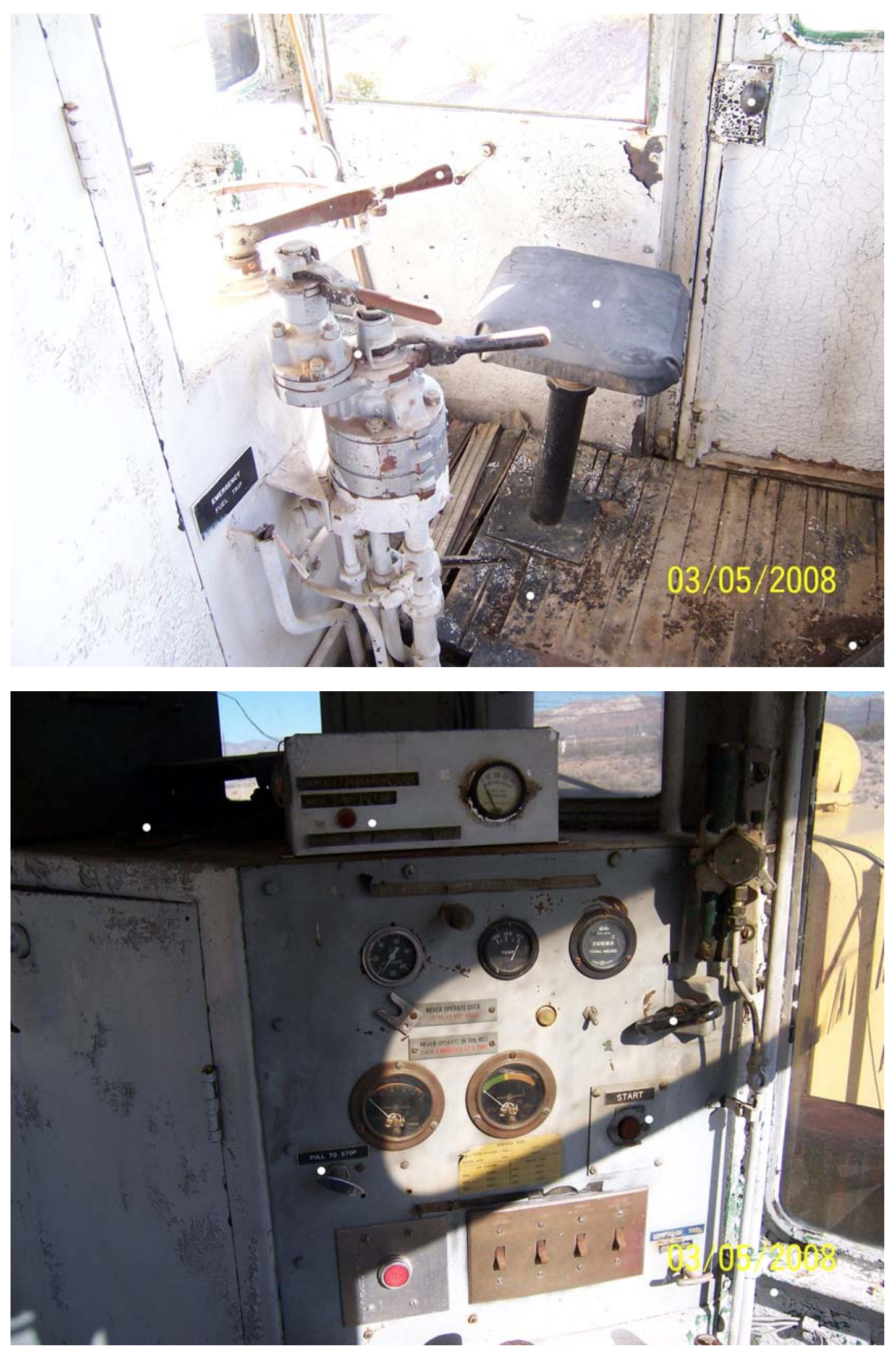
Engine Maintenance, Assembly, and Disassembly (EMAD) Locomotive Survey to Determine Release from Radiological Controls Criteria

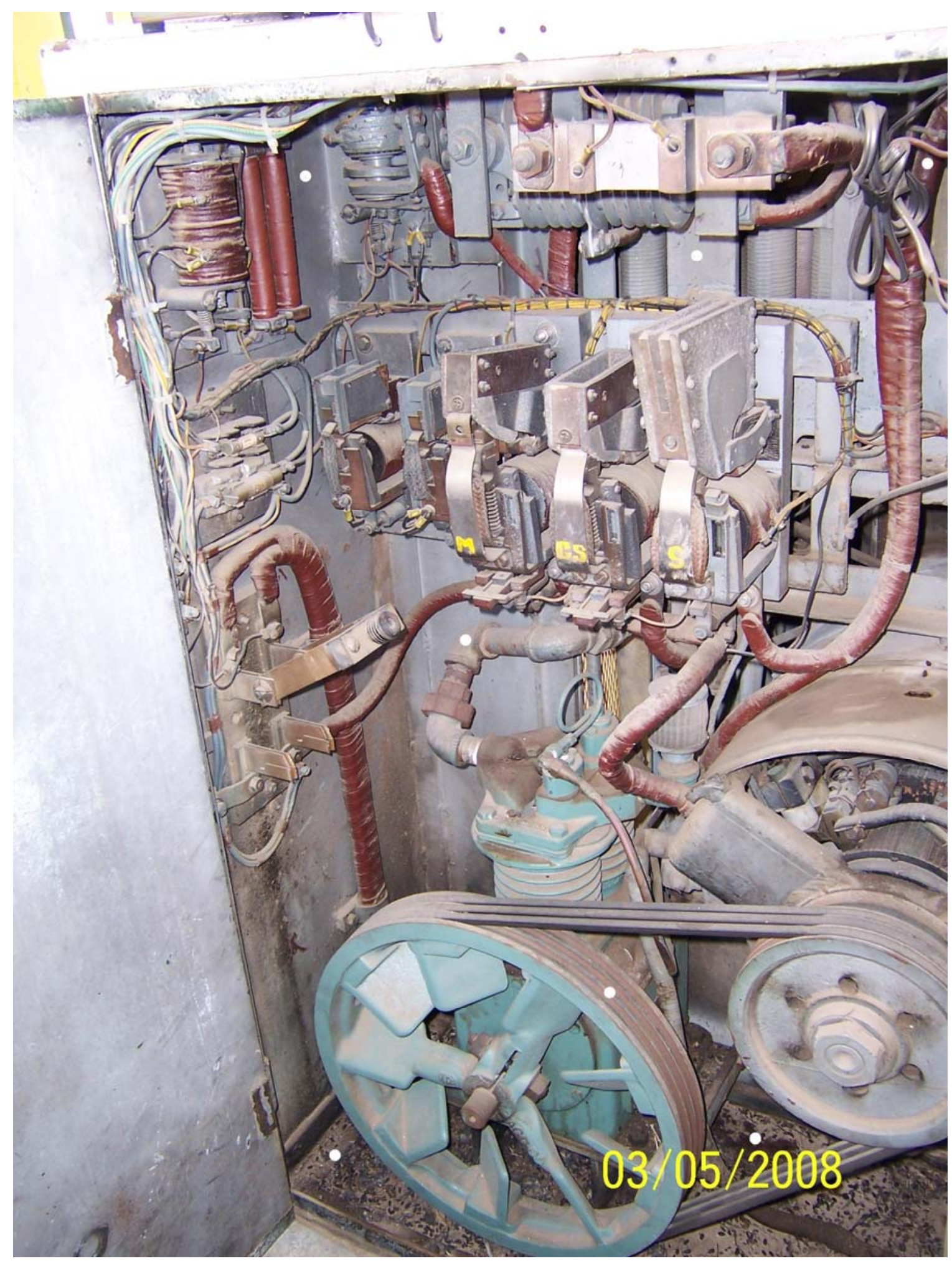


Authorized Limits for Release of the

EM AD 25-Ton Locomotive

May 2010

ATTACHMENT 3

Survey Results \#08-ER-A25-185 for Survey Plan \#08-028 
NSTec

RADIOLOGICAL SURVEY

REPORT - DATA

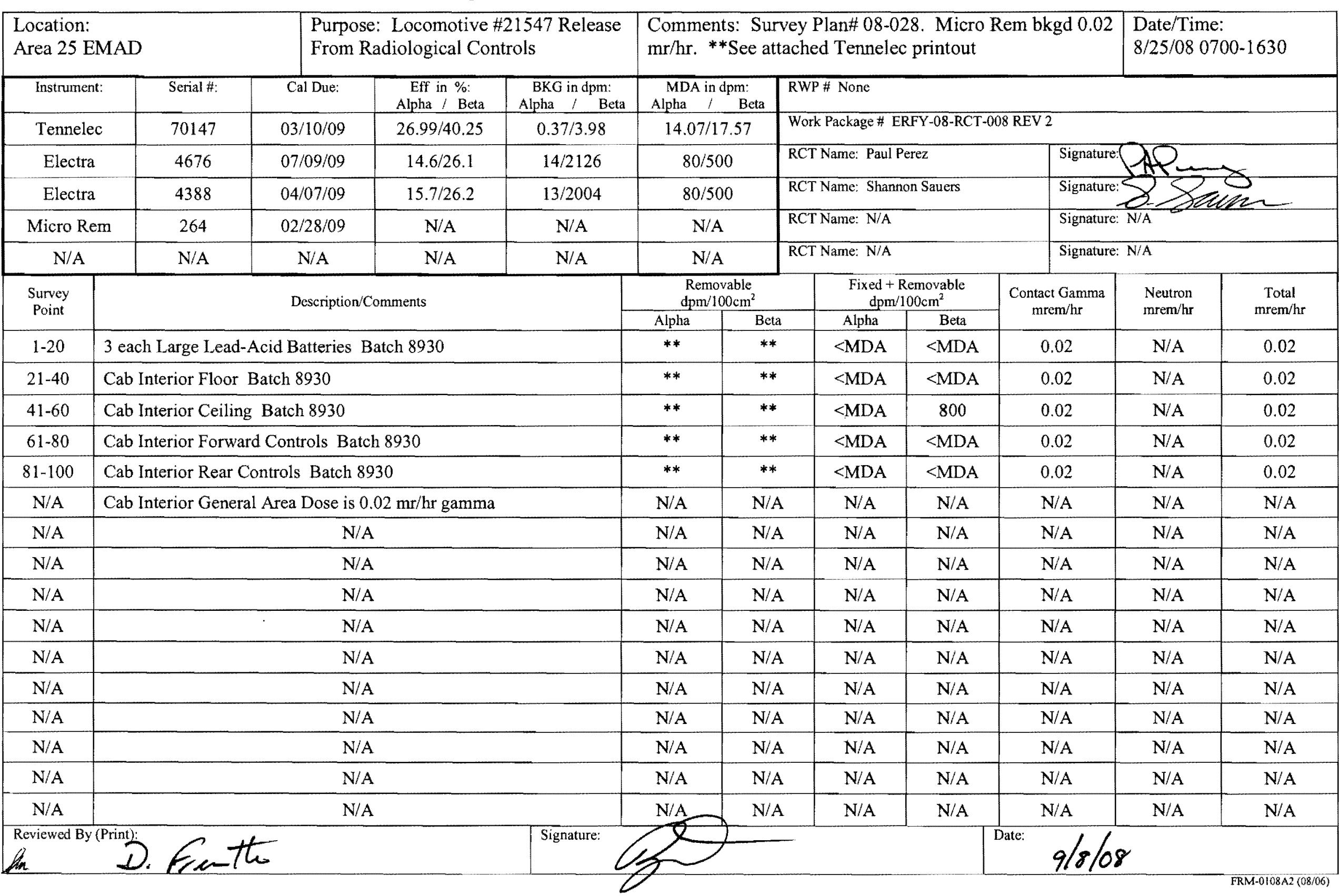


Device: Tennelec 70147

Batch ID: Swipes - 200808251627 Background (dpm) Apha Rate: $\quad 0.37$ Beta Rate:
Efficiency (\%)

Apha: 26.99

Beta: 40.25
MDA (DPM)

Alpha: 14.07

Beta: $\quad 17.57$
Page 2 of 23

Count Date: $\quad$ 8/25/2008 4:27:14PM

Sample Count Timo:

1.00 Minutes

\section{Results Report: Page 1 of 2 Swipes}

Batch Key: 8930

Device:Tennelec 70147

Survey \#OD-ER-A25-185

\section{Sample 10}

20080825162714-A1 20080825162854-A2 20080825163004-A3 20080825163124A4 20080825163244-A5 20080825163354-A6 20080825163514-A7 20080825163634-AB 20080825163744-A9 20080825163904-A10 20080825164024-A11 20080825164134-A12 20080825164254-A13 20080825164414-A14 20080825164524-A15 20080825164644-A16 20080825164804-A17 20080825164914-A18 20080825165034-A19 20080825165154-A20 20080825165314-A21 20080825165424-A22 20080825165544-A23 20080825165704-A24 20080825165814-A25 20080825165934 A26 20080825170054-A27 20080825170204-A28 20080825170324-A29 20080825170444-A30 20080825170554-A31 20080825170714-A32 20080825170834-A33 20080825170944-A34 20080825171104-A35 20080825171224-A36 20080825171334-A37 20080825171454-A38 20080825171614-A39 20080825171724-A40 20080825171844-A41 20080825172004-A42 20080825172124-A43 20080825172234-A44 20080825172354A45 20080825172514-A46 20080825172624-A47 20080825172744-A48 20080825172904-A49 20080825173014-A50

\section{Alpha (dpm)}

$-0.37$

$-0.37$

$-0.37$

$-0.37$

$-0.37$

$-0.37$

$-0.37$

3.33

$-0.37$

3.33

$-0.37$

$-0.37$

$-0.37$

$-0.37$

$-0.37$

$-0.37$

$-0.37$

$-0.37$

$-0.37$

$-0.37$

$-0.37$

3.33

$-0.37$

$-0.37$

3.33

$-0.37$

$-0.37$

$-0.37$

3.33

$-0.37$

$-0.37$

$-0.37$

$-0.37$

$-0.37$

$-0.37$

$-0.37$

$-0.37$

$-0.37$

$-0.37$

$-0.37$

3.33

3.33

$-0.37$

$-0.37$

$-0.37$

$-0.37$

$-0.37$

$-0.37$

$-0.37$

$-0.37$
Bota (dom)

$-1.49$

3.48

8.45

5.96

3.48

0.99

3.48

8.45

3.48

3.48

0.99

0.99

$-1.49$

3.48

$-3.98$

0.99

3.48

$-1.49$

0.99

0.99

$-1.49$

$-3.98$

0.99

$-3.98$

5.96

0.99

$-3.98$

8.45

0.99

$-1.49$

0.99

0.99

$-3.98$

0.99

$-3.98$

0.99

$-1.49$

3.48

$-1.49$

$-3.98$

3.48

3.48

3.48

0.99

$-3.98$

0.99

3.48

3.48

$-3.98$

$-1.49$

\section{Comments}

3 Each Large Lead acid batteries (1-20)
Cab Interior Floor (21-40)

Cab Interior Ceiling (41-60) 
Results Report: Page 2 of 2

Batch Key: 8930
Swipes

Device:Tennelec 70147
Page 3 of 23

\begin{tabular}{|c|c|c|c|}
\hline Sample ID & $\begin{array}{l}\text { Alpha } \\
\text { (dpm) }\end{array}$ & $\frac{\text { Bota }}{\text { (dpm) }}$ & Comments \\
\hline 20080825173134-A51 & -0.37 & -1.49 & \\
\hline 20080825173254-A52 & -0.37 & -1.49 & \\
\hline 20080825173404-A53 & -0.37 & 0.99 & \\
\hline 20080825173524-A54 & -0.37 & -1.49 & \\
\hline $20080825173644-A 55$ & -0.37 & -3.98 & \\
\hline $20080825173754-A 56$ & -0.37 & -3.98 & \\
\hline 20080825173914-A57 & -0.37 & 0.99 & \\
\hline $20080825174034-A 58$ & -0.37 & -1.49 & \\
\hline 20080825174144-A59 & -0.37 & -1.49 & \\
\hline $20080825174304-A 60$ & -0.37 & -1.49 & \\
\hline $20080825174424-A 61$ & -0.37 & -1.49 & Cab Interior FWD Controls $(61-80)$ \\
\hline $20080825174544-A 62$ & -0.37 & 8.45 & \\
\hline 20080825174654-A63 & -0.37 & -1.49 & \\
\hline $20080825174814-A 64$ & -0.37 & -1.49 & \\
\hline 20080825174934-A65 & -0.37 & -1.49 & \\
\hline 20080825175044-A66 & -0.37 & -1.49 & \\
\hline $20080825175204-A 67$ & -0.37 & 10.93 & \\
\hline $20080825175324-A 68$ & -0.37 & 3.48 & \\
\hline $20080825175434-A 69$ & -0.37 & -3.98 & \\
\hline 20080825175554-A70 & -0.37 & -3.98 & \\
\hline $20080825175714-A 71$ & -0.37 & 5.96 & \\
\hline 20080825175824-A72 & -0.37 & 0.99 & \\
\hline 20080825175944-A73 & 3.33 & 3.48 & \\
\hline $20080825180104-A 74$ & -0.37 & 5.96 & \\
\hline 20080825180214-A75 & -0.37 & 0.99 & \\
\hline 20080825180334-A76 & -0.37 & -3.98 & \\
\hline $20080825180454-A 77$ & -0.37 & 5.96 & \\
\hline 20080825180604-A78 & -0.37 & 3.48 & \\
\hline 20080B25180724-A79 & -0.37 & 3.48 & \\
\hline $20080825180844-A 80$ & -0.37 & -3.98 & \\
\hline $20080825181004-A 81$ & -0.37 & 0.99 & Cab Interior Rear Controls $(81-100)$ \\
\hline 20080825181114-A82 & -0.37 & 0.99 & \\
\hline 20080825181234-A83 & 3.33 & 0.99 & \\
\hline 20080825181354-A84 & -0.37 & 0.99 & \\
\hline 20080825181504-A85 & -0.37 & 8.45 & \\
\hline 20080825181624-A86 & -0.37 & -1.49 & \\
\hline 20080825181744-A87 & -0.37 & 0.99 & \\
\hline $20080825181854-A 88$ & -0.37 & 20.87 & \\
\hline $20080825182014-A 89$ & 7.04 & 3.48 & \\
\hline $20080825182134-A 90$ & -0.37 & 0.99 & \\
\hline 200808251822244-A91 & -0.37 & 0.99 & \\
\hline 20080825182404-A92 & -0.37 & -1.49 & \\
\hline 20080825182524-A93 & -0.37 & -3.98 & \\
\hline $20080825182634-A 94$ & -0.37 & 0.99 & \\
\hline 20080825182754-A95 & -0.37 & 0.99 & \\
\hline 20080825182914-A96 & -0.37 & 3.48 & \\
\hline 20080825183024-A97 & -0.37 & -1.49 & \\
\hline 20080825183144-A98 & -0.37 & 0.99 & \\
\hline 20080825183304-A99 & -0.37 & 0.99 & \\
\hline 20080825183424-A100 & -0.37 & 0.99 & \\
\hline
\end{tabular}




\section{RADIOLOGICAL SURVEY \\ REPORT - DATA}

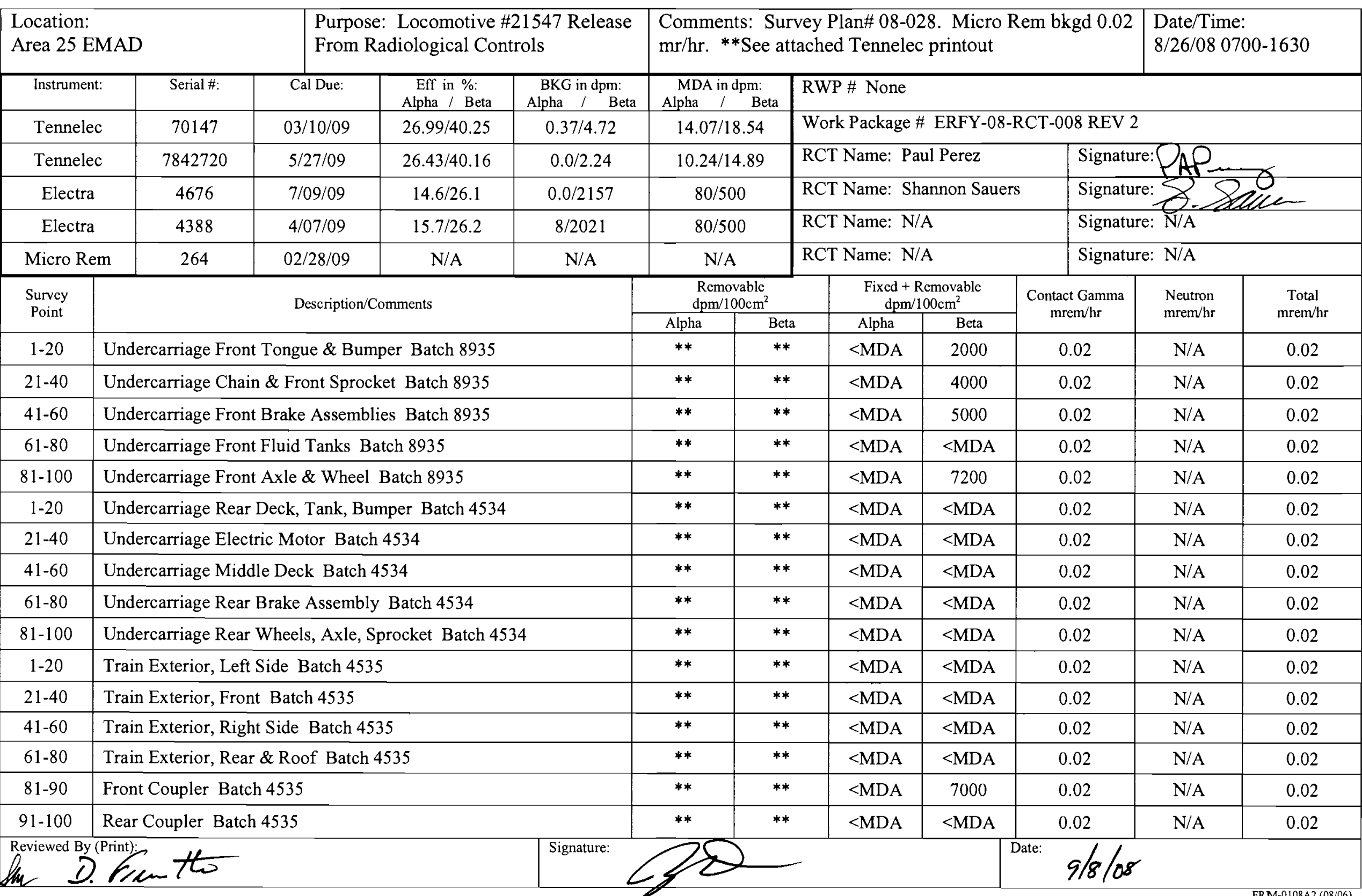

NSTec

SURVEY \# 08-ER-A25-185 Page 4 of 23

Purpose: Locomotive \#21547 Release

ments: Survey Plan\# 08-028. Micro Rem bkgd 0.02

Area 25 EMAD 
NSTec

RADIOLOGICAL SURVEY

REPORT - DATA (CONTINUATION)

SURVEY \# 08-ER-A25-185

\begin{tabular}{|c|c|}
\multicolumn{2}{|c}{$\begin{array}{r}\text { Removable } \\
\text { dpm/100 } \mathrm{cm}^{2}\end{array}$} \\
\hline Alipha & B \\
\hline$* *$ & \\
\hline$* *$ & \\
\hline$* *$ & \\
\hline$* *$ & $* *$ \\
\hline
\end{tabular}

\begin{tabular}{|l|l|l}
\hline $\mathrm{N} / \mathrm{A}$ & $\mathrm{N} / \mathrm{A}$ & $\mathrm{N}$ \\
\hline $\mathrm{N} / \mathrm{A}$ & $\mathrm{N} / \mathrm{A}$ & $\mathrm{N}$ \\
\hline $\mathrm{N} / \mathrm{A}$ & $\mathrm{N} / \mathrm{A}$ & $\mathrm{N}$ \\
\hline $\mathrm{N} / \mathrm{A}$ & $\mathrm{N} / \mathrm{A}$ & $\mathrm{N} / \mathrm{A}$ \\
\hline $\mathrm{N} / \mathrm{A}$ & $\mathrm{N} / \mathrm{A}$ & \\
\hline
\end{tabular}

\begin{tabular}{|c|c|}
\hline $\mathrm{N} / \mathrm{A}$ & $\mathrm{N} / \mathrm{A}$ \\
\hline $\mathrm{N} / \mathrm{A}$ & $\mathrm{N} / \mathrm{A}$ \\
\hline$N / A$ & $\mathrm{~N} / \mathrm{A}$ \\
\hline & $N / A$ \\
\hline
\end{tabular}

\begin{tabular}{|l|l}
\hline $\mathrm{N} / \mathrm{A}$ & $\mathrm{N} / \mathrm{A}$ \\
\hline $\mathrm{N} / \mathrm{A}$ & $\mathrm{N} / \mathrm{A}$ \\
\hline $\mathrm{N} / \mathrm{A}$ & $\mathrm{N} / \mathrm{A}$ \\
\hline
\end{tabular}

\begin{tabular}{|c|c|}
\hline $\mathrm{N} / \mathrm{A}$ & $\mathrm{N} / \mathrm{A}$ \\
\hline $\mathrm{N} / \mathrm{A}$ & $\mathrm{N} / \mathrm{A}$ \\
\hline $\mathrm{N} / \mathrm{A}$ & $\mathrm{N} / \mathrm{A}$ \\
\hline $\mathrm{N} / \mathrm{A}$ & $\mathrm{N} / \mathrm{A}$ \\
\hline $\mathrm{N} / \mathrm{A}$ & $\mathrm{N} / \mathrm{A}$ \\
\hline $\mathrm{N} / \mathrm{A}$ & $\mathrm{N} / \mathrm{A}$ \\
\hline $\mathrm{N} / \mathrm{A}$ & $\mathrm{N} / \mathrm{A}$ \\
\hline $\mathrm{N} / \mathrm{A}$ & $\mathrm{N} / \mathrm{A}$ \\
\hline $\mathrm{N} / \mathrm{A}$ & $\mathrm{N} / \mathrm{A}$ \\
\hline $\mathrm{N} / \mathrm{A}$ & $\mathrm{N} / \mathrm{A}$ \\
\hline
\end{tabular}

\begin{tabular}{|c|c|c|c|c|}
\hline \multicolumn{2}{|c|}{$\begin{array}{l}\text { Fixed + Removable } \\
\mathrm{dpm} / 100 \mathrm{~cm}^{2}\end{array}$} & \multirow{2}{*}{$\begin{array}{l}\text { Gamma } \\
\text { mrem/hr }\end{array}$} & \multirow{2}{*}{$\begin{array}{l}\text { Neutron } \\
\mathrm{mrem} / \mathrm{hr}\end{array}$} & \multirow{2}{*}{$\begin{array}{l}\text { Total } \\
\mathrm{mrem} / \mathrm{hr}\end{array}$} \\
\hline Alpha & Beta & & & \\
\hline$<\mathrm{MDA}$ & $<\mathrm{MDA}$ & 0.02 & $\mathrm{~N} / \mathrm{A}$ & 0.02 \\
\hline$\angle \mathrm{MDA}$ & $<\mathrm{MDA}$ & 0.02 & N/A & 0.02 \\
\hline$<\mathrm{MDA}$ & 10,800 & 0.02 & $\mathrm{~N} / \mathrm{A}$ & 0.02 \\
\hline$<\mathrm{MDA}$ & $<\mathrm{MDA}$ & 0.02 & N/A & 0.02 \\
\hline N/A & $\mathrm{N} / \mathrm{A}$ & $\mathrm{N} / \mathrm{A}$ & N/A & $\mathrm{N} / \mathrm{A}$ \\
\hline N/A & N/A & $\mathrm{N} / \mathrm{A}$ & N/A & $\mathrm{N} / \mathrm{A}$ \\
\hline $\mathrm{N} / \mathrm{A}$ & $\mathrm{N} / \mathrm{A}$ & $\mathrm{N} / \mathrm{A}$ & N/A & $\mathrm{N} / \mathrm{A}$ \\
\hline $\mathrm{N} / \mathrm{A}$ & $\mathrm{N} / \mathrm{A}$ & N/A & $\mathrm{N} / \mathrm{A}$ & $\mathrm{N} / \mathrm{A}$ \\
\hline N/A & $\mathrm{N} / \mathrm{A}$ & $\mathrm{N} / \mathrm{A}$ & $\mathrm{N} / \mathrm{A}$ & N/A \\
\hline $\mathrm{N} / \mathrm{A}$ & $\mathrm{N} / \mathrm{A}$ & N/A & N/A & $\mathrm{N} / \mathrm{A}$ \\
\hline $\mathrm{N} / \mathrm{A}$ & $\mathrm{N} / \mathrm{A}$ & N/A & N/A & N/A \\
\hline N/A & N/A & N/A & $\mathrm{N} / \mathrm{A}$ & $\mathrm{N} / \mathrm{A}$ \\
\hline N/A & $\mathrm{N} / \mathrm{A}$ & $\mathrm{N} / \mathrm{A}$ & N/A & $\mathrm{N} / \mathrm{A}$ \\
\hline N/A & N/A & $\mathrm{N} / \mathrm{A}$ & N/A & N/A \\
\hline $\mathrm{N} / \mathrm{A}$ & $\mathrm{N} / \mathrm{A}$ & N/A & N/A & $\mathrm{N} / \mathrm{A}$ \\
\hline N/A & N/A & N/A & N/A & N/A \\
\hline $\mathrm{N} / \mathrm{A}$ & N/A & $\mathrm{N} / \mathrm{A}$ & N/A & N/A \\
\hline $\mathrm{N} / \mathrm{A}$ & $\mathrm{N} / \mathrm{A}$ & $\mathrm{N} / \mathrm{A}$ & $\mathrm{N} / \mathrm{A}$ & $\mathrm{N} / \mathrm{A}$ \\
\hline N/A & $\mathrm{N} / \mathrm{A}$ & $\mathrm{N} / \mathrm{A}$ & $\mathrm{N} / \mathrm{A}$ & $\mathrm{N} / \mathrm{A}$ \\
\hline N/A & N/A & N/A & N/A & $\mathrm{N} / \mathrm{A}$ \\
\hline $\mathrm{N} / \mathrm{A}$ & $\mathrm{N} / \mathrm{A}$ & N/A & N/A & $\mathrm{N} / \mathrm{A}$ \\
\hline N/A & N/A & $\mathrm{N} / \mathrm{A}$ & $\mathrm{N} / \mathrm{A}$ & $\mathrm{N} / \mathrm{A}$ \\
\hline N/A & $\mathrm{N} / \mathrm{A}$ & $\mathrm{N} / \mathrm{A}$ & N/A & $\mathrm{N} / \mathrm{A}$ \\
\hline N/A & N/A & $\mathrm{N} / \mathrm{A}$ & N/A & $\mathrm{N} / \mathrm{A}$ \\
\hline
\end{tabular}


Device: Tennelec 70147

Batch ID: Swipes - 200808260644

Background (dpm)

Apha Rate: $\quad 0.37$

Beta Rate:

\begin{abstract}
4.72
\end{abstract}
Efficiency (\%)

Apha: 26.99

Beta: 40.25
MDA (DPM)

Apha: 14.07
Page 6 of 23

Count Date: $\quad 8 / 26 / 2008$ 6:44:09AM

Sample Count Time: 1.00 Minutes

Results Roport: Page 1 of 2 Swipes

Batch Koy: 8935

\section{Sample ID}

20080826064409-A1 20080826064550-A2 $20080826064700-A 3$ 20080826064820-A4 20080826064940-A5 20080826065050-A6 20080826065210-A7 20080826065330-A8 20080826065440-A9 $20080826065600-A 10$ 20080826065720-A11 20080826065830-A12 20080826065950-A13 20080826070110-A14 20080826070220-A15 20080826070340-A16 20080826070500-A17 20080826070610-A18 20080826070730-A19 20080826070850-A20 20080826071010-A21 20080826071120-A22 20080826071240-A23 20080826071400-A24 20080826071510-A25 20080826071630-A26 20080826071750-A27 20080826071900-A28 20080826072020-A29 20080826072140-A30 20080826072250-A31 20080826072410-A32 20080826072530-A33 20080826072640-A34 20080826072800-A35 20080826072920-A36 20080826073040-A37 20080826073150-A38 20080826073310-A39 20080826073430-A40 20080826073540-A41 20080826073700-A42 20080826073820-A43 20080826073930-A44 20080826074050-A45 20080826074210-A46 20080826074320-A47 20080826074440-A48 20080826074600-A49 20080826074720-A50
Device:Tennelec 70147

Survey \#_O8-ER-A25-185

\section{Beta \\ (dom) Comments}

$-0.37 \quad 5.22$

$\begin{array}{ll}-0.37 & 0.25\end{array}$

$3.33 \quad-4.72$

$\begin{array}{ll}-0.37 & 7.70\end{array}$

$\begin{array}{ll}-0.37 & 0.25\end{array}$

$-0.37 \quad-2.24$

$-0.37 \quad-2.24$

$-0.37 \quad-2.24$

$3.33 \quad 0.25$

$3.33 \quad 0.25$

$\begin{array}{ll}-0.37 & -2.24\end{array}$

$-0.37 \quad-4.72$

$\begin{array}{ll}-0.37 & 0.25\end{array}$

$-0.37 \quad-2.24$

$-0.37 \quad 2.73$

$\begin{array}{ll}-0.37 & 5.22\end{array}$

$\begin{array}{ll}-0.37 & 0.25\end{array}$

$3.33 \quad-2.24$

$\begin{array}{ll}-0.37 & -2.24\end{array}$

$3.33 \quad 0.25$

$\begin{array}{ll}-0.37 & -4.72 \\ -0.37 & -2.24\end{array}$

$-0.37 \quad-2.24$

$3.33 \quad 0.25$

$\begin{array}{ll}-0.37 & 7.70\end{array}$

$-0.37 \quad-2.24$

$3.33 \quad 0.25$

$\begin{array}{ll}-0.37 & 0.25\end{array}$

$-0.37 \quad-4.72$

$\begin{array}{ll}-0.37 & 0.25\end{array}$

$3.33 \quad 0.25$

$3.33 \quad 2.73$

$\begin{array}{ll}-0.37 & 5.22\end{array}$

$\begin{array}{ll}-0.37 & 0.25\end{array}$

$\begin{array}{ll}-0.37 & 0.25\end{array}$

$\begin{array}{ll}-0.37 & 5.22 \\ 3.33 & -2.24\end{array}$

$3.33-2.24$

$\begin{array}{ll}-0.37 & 2.73\end{array}$

$3.33 \quad 20.12$

$3.33 \quad 5.22$

$\begin{array}{ll}-0.37 & -4.72\end{array}$

$\begin{array}{ll}-0.37 & 12.67\end{array}$

$\begin{array}{ll}-0.37 & 15.16\end{array}$

$\begin{array}{ll}-0.37 & 0.25\end{array}$

$3.33 \quad 2.73$

$\begin{array}{ll}-0.37 & 0.25\end{array}$

$\begin{array}{ll}-0.37 & 5.22\end{array}$

$\begin{array}{ll}-0.37 & 0.25\end{array}$

$\begin{array}{ll}-0.37 & 7.70\end{array}$

$-0.37 \quad-2.24$

$\begin{array}{ll}-0.37 & 0.25\end{array}$
Undercarriage, Front Tongue and Bumper (1-20)

Undercarriage, Drive Chain and Sprockets $(21-40)$ 
Results Report: Page 2 of 2

Batch Key: 8935
Swipes

Device:Tennelec 70147
Page 7 of 23

\begin{tabular}{|c|c|c|c|}
\hline Sample ID & $\begin{array}{l}\text { Alpha } \\
\text { (dpm) }\end{array}$ & $\frac{\text { Beta }}{\text { (dom) }}$ & Comments \\
\hline 20080826074830-A51 & -0.37 & 0.25 & \\
\hline $20080826074950-A 52$ & 7.04 & 5.22 & \\
\hline $20080826075110-A 53$ & 7.04 & 0.25 & \\
\hline $20080826075220-A 54$ & -0.37 & 5.22 & \\
\hline 20080826075340-A55 & -0.37 & -2.24 & \\
\hline $20080826075500-A 56$ & -0.37 & 5.22 & \\
\hline $20080826075610-A 57$ & -0.37 & 0.25 & \\
\hline $20080826075730-A 58$ & 3.33 & 7.70 & \\
\hline $20080826075850-A 59$ & -0.37 & 0.25 & \\
\hline $20080826080000-A 60$ & -0.37 & -4.72 & \\
\hline $20080826080120-A 61$ & -0.37 & 0.25 & Undercarriage, Front Fluid Tanks $(61-80)$ \\
\hline 20080826080240-A62 & -0.37 & 2.73 & \\
\hline 20080826080400-A63 & -0.37 & 12.67 & \\
\hline $20080826080510-A 64$ & -0.37 & 7.70 & \\
\hline $20080826080630-A 65$ & -0.37 & 2.73 & \\
\hline $20080826080750-A 66$ & -0.37 & 0.25 & \\
\hline 20080826080900-A67 & 3.33 & 0.25 & \\
\hline $20080826081020-A 68$ & -0.37 & -2.24 & \\
\hline 20080826081140-A69 & 3.33 & 0.25 & \\
\hline $20080826081250-A 70$ & 3.33 & 2.73 & \\
\hline 20080826081410-A71 & -0.37 & 7.70 & \\
\hline 20080826081530-A72 & -0.37 & 0.25 & \\
\hline 20080826081640-A73 & -0.37 & 2.73 & \\
\hline 20080826081800-A74 & -0.37 & -2.24 & \\
\hline 20080826081920-A75 & -0.37 & 2.73 & \\
\hline $20080826082030-A 76$ & -0.37 & 2.73 & \\
\hline 20080826082150-A77 & -0.37 & 5.22 & \\
\hline $20080826082310-A 78$ & -0.37 & 2.73 & \\
\hline 20080826082430-A79 & -0.37 & 2.73 & \\
\hline $20080826082540-A 80$ & -0.37 & -4.72 & \\
\hline 20080826082700-A81 & -0.37 & 2.73 & Undercarriage, Front Wheel Assembly and Axle (81-100) \\
\hline $20080826082820-A B 2$ & -0.37 & 0.25 & \\
\hline $20080826082930-A 83$ & 3.33 & -2.24 & \\
\hline 20080826083050-A84 & 7.04 & 7.70 & \\
\hline $20080826083210-A 85$ & 3.33 & 0.25 & \\
\hline 20080826083320-A86 & -0.37 & 2.73 & \\
\hline 20080826083440-A87 & -0.37 & -2.24 & \\
\hline $20080826083600-A 88$ & -0.37 & 7.70 & \\
\hline 20080826083710-A89 & -0.37 & 0.25 & \\
\hline $20080826083830-A 90$ & -0.37 & -4.72 & \\
\hline 20080826083950-A91 & -0.37 & -4.72 & \\
\hline 20080826084100-A92 & -0.37 & 5.22 & \\
\hline 20080826084220-A93 & -0.37 & -2.24 & \\
\hline $20080826084340-A 94$ & -0.37 & 7.70 & \\
\hline 20080826084450-A95 & 3.33 & 10.19 & \\
\hline 20080826084610-A96 & -0.37 & 2.73 & \\
\hline 20080B26084730-A97 & -0.37 & 5.22 & \\
\hline $20080826084850-A 98$ & 3.33 & -2.24 & \\
\hline 20080826085000-A99 & -0.37 & 2.73 & \\
\hline $20080826085120-A 100$ & -0.37 & 0.25 & \\
\hline
\end{tabular}


Device: SMS ID: $\mathbf{7 8 4 2 7 2 0}$

Batch ID: Swipes - 200808261342
Page 8 of 23

Count Date: $\quad 8 / 26 / 2008$ 1:42:27PM
Background (dpm) Apha Rate: 0.00 2.24
Efficiency (\%)

Alpha: 26.43

Beta: 40.16
MDA (DPM)

Alpha: 10.24

Beta: $\quad 14.89$
Sample Count Time:

1.00 Minutes

\section{Results Report: Page 1 of $2 \quad$ Swipes}

Batch Key: 4534

Device:SMS ID: 7842720

Survey \# O8-ER-A25-185

Sample ID

20080826134227-A1 20080826134528-A2 20080826134638-A3 20080826134758-A4 20080826134908-A5 20080826135018-A6 20080826135128-A7 20080826135248-A8 20080826135358-A9 20080826135508-A10 20080826135628-A11 20080826135738-A12 20080826135848-A13 20080826140008-A14 20080826140118-A15 20080826140228-A16 20080826140338-A17 20080826140458-A18 20080826140608-A19 20080826140718-A20 20080826140838-A21 20080826140948-A22 20080826141058-A23 20080826141208-A24 20080826141328-A25 20080826141438-A26 20080826141548-A27 20080826141708-A28 20080826141818-A29 20080826141928-A30 20080826142048-A31 20080826142158-A32 20080826142308-A33 20080826142418-A34 20080826142538-A35 20080826142648-A36 20080826142758-A37 20080826142918-A38 20080826143028-A39 20080826143138-A40 20080826143248-A41 20080826143408-A42 20080826143518-A43 20080826143628-A44 20080826143748-A45 20080826143858-A46 20080826144008-A47 20080826144118-A48 20080826144238-A49 20080826144348-A50 20080826144458-A51 20080826144618-A52 20080826144728-A53

\section{Alpha}

(dpm)

0

3.78

3.78

3.78

0

7.57

0

0

0

3.78

0

o

0

3.78

7.57

0

0

0

0

0

0

o

0

0

0

0

3.78

0

3.78

0

0

0

0

3.78

0

0

3.78

0

0

0

3.78

0

0

0

0

o

0

0

3.78

0

0

0

o

\section{Beta} (dpm)

\subsection{3}

0.25

$-2.24$

10.21

0.25

0.25

10.21

0.25

$-2.24$

2.74

2.74

0.25

5.23

7.72

5.23

2.74

2.74

7.72

7.72

0.25

2.74

$-2.24$

5.23

2.74

0.25

2.74

10.21

0.25

2.74

0.25

2.74

2.74

0.25

7.72

2.74

$-2.24$

2.74

$-2.24$

2.74

10.21

5.23

0.25

0.25

0.25

2.74

2.74

2.74

2.74

0.25

0.25

2.74

0.25

2.74

\section{Comments}

Rear Deck, Fluid Tank, Bumper Undercarriage (1-20)
Electric Motor (21-40)

Middle of Train Deck Bottom, Undercarriage (41-60) 
O8-ER-A25-185

\section{Sample ID}

20080826144838-A54 20080826144948-A55 20080826145108-A56 20080826145218-A57 20080826145328-A58 20080826145448-A59 20080826145558-A60 20080826145708-A61 20080826145818-A62 20080826145938-A63 20080826150048-A64 20080826150158-A65 20080826150318-A66 20080826150428-A67 20080826150538-A68 20080826150658-A69 20080826150808-A70 20080826150918-A71 20080826151028-A72 20080826151148-A73 20080826151258-A74 20080826151408-A75 20080826151528-A76 20080826151638-A77 20080826151748-A78 20080826151858-A79 20080826152018-A80 20080826152128-A81 20080826152238-AB2 20080826152358-A83 20080826152508-A84 20080826152618-A85 20080826152738-A86 20080826152848-A87 20080826152958-A88 20080826153108-A89 20080826153229-A90 20080826153338-A91 20080826153448-A92 20080826153559-A93 20080826153718-A94 20080826153828-A95 20080826153938-A96 20080826154058-A97 20080826154208-A98 20080826154318-A99 20080826154438-A100

\section{Alpha}

(dom)

0

3.78

0

0

0

0

3.78

0

o

o

0

0

o

o

0

3.78

0

3.78

0

0

0

o

0

0

o

0

0

0

0

0

0

0

0

0

3.78

0

0

o

0

0

0

0

3.78

0

0

0

0
Beta

(dpm)

2.74

7.72

0.25

$-2.24$

2.74

$-2.24$

2.74

0.25

$-2.24$

5.23

0.25

2.74

$-2.24$

$-2.24$

5.23

0.25

2.74

0.25

0.25

5.23

0.25

2.74

2.74

5.23

$-2.24$

0.25

2.74

$-2.24$

2.74

0.25

0.25

0.25

0.25

$-2.24$

10.21

5.23

5.23

2.74

$-2.24$

0.25

$-2.24$

$-2.24$

2.74

0.25

7.72

2.74

0.25
Rear Brake Assemblies (61-80)

Rear Wheels, Axel, Drive Sprocket, Undercarriage (81-100) 
Device: SMS ID: 7842720

Batch 1D: Swipes - 200808261549
Page 10 of 23

Count Date: $\quad$ 8/26/2008 3:49:27PM
Background (dpm)

Apha Rate:

0.00

2.24

Beta Rate:
Efficiency (\%)

Alpha: 26.43

Beta: 40.16
MDA (DPM)

Alpha: $\quad 10.24$
Sample Count Time:

1.00 Minutes

Results Report: Page 1 of 2

Swipes

Batch Key: 4535

Device:SMS ID: 7842720

Survey \#O8-ER-A25-185

\section{Sample ID}

20080826154927-A1

20080826155058-A2

20080826155208-A3

20080826155328-A4

20080826155438-A5

20080826155548-A6

20080826155708-A7

2008082615581B-AB

20080826155928-A9

20080826160038-A10

20080826160158-A11

20080826160308-A12

20080826160418-A13

20080826160538-A14

20080826160648-A15

20080826160758-A16

20080826160908-A17

20080826161028-A18

20080826161138-A19

20080826161248-A20

20080826161408-A21

20080826161518-A22

20080826161628-A23

20080826161748-A24

20080826161858-A25

20080826162008-A26

20080826162118-A27

20080826162238-A28

20080826162348-A29

20080826162458-A30

20080826162618-A31

20080826162728-A32

20080826162838-A33

20080826162948-A34

20080826163108-A35

20080826163218-A36

20080826163328-A37

20080826163448-A38

20080826163558-A39

20080826163708-A40

20080826163828-A41

200808261639338-A42

20080826164048-A43

20080826164158-A44

20080826164318-A45

20080826164428-A46 20080826164538-A47

20080826164658-A48

20080826164808-A49

20080826164918-A50

20080826165028-A51

20080826165148-A52

20080826165258-A53

\section{Alpha}

(dpm)

3.78

0

0

0

0

0

3.78

0

0

0

0

3.78

3.78

3.78

0

3.78

0

0

0

0

0

0

0

0

0

0

0

0

0

0

0

0

0

0

0

0

3.78

0

3.78

0

0

0

0

0

0

0

3.78

3.78

0

0

0

0

\section{Beta}

(dpm)

2.74

$-2.24$

$-2.24$

$-2.24$

0.25

0.25

2.74

12.70

2.74

0.25

0.25

5.23

5.23

$-2.24$

2.74

7.72

0.25

$-2.24$

10.21

7.72

$-2.24$

2.74

7.72

0.25

5.23

$-2.24$

2.74

0.25

$-2.24$

0.25

2.74

$-2.24$

2.74

0.25

2.74

$-2.24$

0.25

$-2.24$

0.25

2.74

2.74

2.74

0.25

0.25

2.74

5.23

5.23

7.72

0.25

0.25

$-2.24$

0.25

2.74

\section{Comments}

Train Exterior, Left Side (1-20)

PAP s/ados

Train Exterior, Front Cotplef (21-40)
Train Exterior, Right Side (41-60) 


\section{Sample 1D}

20080826165408-A54 20080826165528-A55 20080826165638-A56 20080826165748-A57 20080826165908-A58 20080826170018-A59 20080826170128-A60 20080826170238-A61 20080826170358-A62 20080826170508-A63 20080826170618-A64 20080826170738-A65 20080826170848-A66 20080826170958-A67 20080826171108-A68 20080826171228-A69 20080826171338-A70 20080826171448-A71 20080826171608-A72 20080826171718-A73 20080826171828-A74 20080826171948-A75 20080826172058-A76 20080826172208-A77 20080826172318-A78 20080826172438-A79 20080826172548-A80 20080826172658-A81 20080826172818-AB2 20080826172928-AB3 20080826173038-A84 20080826173148-A85 20080826173308-A86 20080826173418-AB7 20080826173528-A88 20080826173648-A89 20080826173758-A90 20080826173908-A91 20080826174028-A92 20080826174138-A93 20080826174248-A94 20080826174358-A95 20080826174518-A96 20080826174628-A97 20080826174738-A98 20080826174858-A99 20080826175008-A100

\section{Alpha}

(dpm)

0

0

0.25

$0 \quad 0.25$

$0 \quad-2.24$

$0 \quad 5.23$

$3.78 \quad 10.21$

$\begin{array}{ll}3.78 & 7.72\end{array}$

$0 \quad 5.23$

$0 \quad 2.74$

$0 \quad 0.25$

$\begin{array}{ll}3.78 & 0.25\end{array}$

$\begin{array}{ll}3.78 & 7.72\end{array}$

$\begin{array}{ll}0 & 2.74\end{array}$

$11.35 \quad 2.74$

$3.78 \quad 17.68$

$0 \quad 5.23$

$0 \quad 0.25$

$\begin{array}{ll}3.78 & 0.25\end{array}$

$\begin{array}{ll}0 & 7.72\end{array}$

$\begin{array}{ll}7.57 & 0.25\end{array}$

$0 \quad-2.24$

$0 \quad 5.23$

$0 \quad 0.25$

$\begin{array}{ll}0 & 0.25\end{array}$

$0 \quad 2.74$

$\begin{array}{ll}0 & 2.74\end{array}$

$11.35 \quad 0.25$

$3.78-2.24$

$0 \quad 0.25$

$0 \quad 0.25$

$0 \quad 5.23$

0

0 2.74

$0 \quad-2.24$

$3.78 \quad 2.74$

$\begin{array}{ll}0 & 10.21\end{array}$

$\begin{array}{ll}3.78 & 0.25\end{array}$

$0 \quad 5.23$

$0 \quad 2.74$

$3.78 \quad 0.25$

$\begin{array}{ll}0 & 0.25\end{array}$

$3.78-2.24$

$3.78 \quad 0.25$

$0 \quad 10.21$

$0 \quad 0.25$

$0 \quad 2.74$

\section{Comments}

Train Exterior, Rear and Roof (61-80)

Front Coupler (81-90)

Rear Coupler (91-100) 
Device: Tennelec 70147

Batch ID: Swipes - 200808261327

Background (dpm)

Apha Rate: $\quad 0.37$

Beta Rate:

4.72

Efficiency $(\%)$

Apha: 26.99

Beta: 40.25
MDA (DPM)

Alpha: 14.07

Beta: 18.54
Page 12 of 23

Count Date: $\quad$ 8/26/2008 1:27:38PM

Sample Count Time: 1.00 Minutes

\section{Results Report: Page 1 of $3 \quad$ Swipes}

Batch Key: 8937

Device:Tennelec 70147

Survey \# O8-ER-A25-185

\section{Sample 1D}

20080826132738-A1 20080826133039-A2 20080826133159-A3 20080826133319-A4 20080826133439-A5 20080826133549-A6 20080826133709-A7 20080826133829-A8 20080826133939-A9 20080826134059-A10 20080826134219-A11 $20080826134329-A 12$ 20080826134449-A13 20080826134609-A14 20080826134719-A15 20080826134839-A16 20080826134959-A17 20080826135109-A18 20080826135229-A19 20080826135349-A20 20080826135459-A21 20080826135619-A22 20080826135739-A23 20080826135859-A24 20080826140009-A25 20080826140129-A26 20080826140249-A27 20080826140359-A28 20080826140519-A29 20080826140639-A30 20080826140749-A31 20080826140909-A32 20080826141029-A33 20080826141139-A34 20080826141259-A35 20080826141419-A36 20080826141529-A37 20080826141649-A38 $20080826141809-A 39$ 20080826141919-A40 20080826142039-A41 20080826142159-A42 20080826142319-A43 20080826142429-A44 20080826142549-A45 20080826142709-A46 20080826142819-A47 20080826142939-A48 20080826143059-A49 20080826143209-A50

Alpha

$-0.37$

$-0.37$

3.33

$-0.37$

$-0.37$

3.33

$-0.37$

$-0.37$

$-0.37$

$-0.37$

$-0.37$

$-0.37$

$-0.37$

$-0.37$

$-0.37$

$-0.37$

$-0.37$

10.74

$-0.37$

$-0.37$

$-0.37$

$-0.37$

$-0.37$

$-0.37$

$-0.37$

3.33

$-0.37$

$-0.37$

$-0.37$

$-0.37$

$-0.37$

$-0.37$

$-0.37$

$-0.37$

$-0.37$

3.33

$-0.37$

$-0.37$

$-0.37$

$-0.37$

3.33

3.33

$-0.37$

$-0.37$

$-0.37$

3.33

$-0.37$

3.33

$-0.37$

$-0.37$

\section{Beta}

(dpm)

2.73

5.22

0.25

$-2.24$

0.25

0.25

$-2.24$

$-4.72$

5.22

$-4.72$

0.25

0.25

$-2.24$

$-4.72$

$-2.24$

$-2.24$

5.22

2.73

0.25

0.25

$-2.24$

$-2.24$

$-4.72$

$-2.24$

$-2.24$

2.73

5.22

2.73

0.25

$-4.72$

0.25

2.73

0.25

0.25

0.25

0.25

0.25

0.25

2.73

$-4.72$

7.70

0.25

$-2.24$

0.25

$-2.24$

0.25

$-2.24$

0.25

2.73

2.73
Comments

Cab Interior walls $(1-60)$ 


\section{Sample ID}

20080826143329-A51 20080826143449-A52 20080826143559-A53 20080826143719-A54 20080826143839-A55 20080826143949-A56 20080826144109-A57 20080826144229-A58 20080826144339-A59 20080826144459-A60 20080826144619-A61 20080826144739-A62 20080826144849-A63 20080826145009-A64 20080826145129-A65 20080826145239-A66 20080826145359-A67 20080826145519-A68 20080826145629-A69 20080826145749-A70 20080826145909-A71 20080826150019-A72 20080826150139-A73 20080826150259-A74 20080826150409-A75 20080826150529-A76 20080826150649-A77 20080826150759-A78 20080826150919-A79 20080826151039-A80 20080826151159-A81 20080826151309-A82 20080826151429-A83 20080826151549-A84 20080826151659-A85 20080826151819-AB6 20080826151939-A87 20080826152049-A88 20080826152209-A89 20080826152329-A90 20080826152439-A91 20080826152559-A92 20080826152719-A93 20080826152839-A94 20080826152949-A95 20080826153109-A96 20080826153229-A97 20080826153339-A98 20080826153459-A99 20080826153619A100 20080826153729-A1 20080826153849-A2 20080826154009-A3 20080826154119-A4 20080826154239-A5 $20080826154359-A 6$ $20080826154509-A 7$ 20080826154629-A8

Alpha
(dpm)

(dpm)

$-0.37$

$-0.37$

$-0.37$

3.33

3.33

$-0.37$

$-0.37$

$-0.37$

$-0.37$

$-0.37$

$-0.37$

3.33

$-0.37$

$-0.37$

$-0.37$

$-0.37$

$-0.37$

$-0.37$

$-0.37$

$-0.37$

$-0.37$

$-0.37$

$-0.37$

$-0.37$

$-0.37$

$-0.37$

$-0.37$

$-0.37$

$-0.37$

$-0.37$

$-0.37$

$-0.37$

$-0.37$

$-0.37$

$-0.37$

$-0.37$

$-0.37$

$-0.37$

3.33

$-0.37$

$-0.37$

$-0.37$

$-0.37$

$-0.37$

$-0.37$

$-0.37$

$-0.37$

$-0.37$

3.33

$-0.37$

$-0.37$

$-0.37$

3.33

$-0.37$

3.33

$-0.37$

$-0.37$

$-0.37$

\section{Bota}

(dpm)

5.22

$-4.72$

5.22

$-2.24$

5.22

0.25

5.22

7.70

2.73

2.73

$-2.24$

5.22

2.73

$-2.24$

10.19

$-4.72$

5.22

$-2.24$

2.73

$-2.24$

$-2.24$

0.25

$-2.24$

0.25

0.25

0.25

5.22

2.73

$-2.24$

0.25

5.22

0.25

$-2.24$

2.73

2.73

0.25

$-4.72$

$-2.24$

0.25

$-4.72$

2.73

$-2.24$

$-2.24$

0.25

0.25

$-2.24$

5.22

$-2.24$

0.25

2.73

7.70

5.22

2.73

$-2.24$

$-4.72$

$-4.72$

$-4.72$

$-4.72$

\section{Comments}

Cab Interior Electrical Cabinet (61-80)

Right Front Platform and Inspection Ports (81-100)
Left Front Platform (101-109) 
Results Report: Page 3 of 3

Swipes

Page 14 of 23

Batch Koy: 8937

Device:Tennelec 70147

O8-ER-A25-185

Alpha

(dpm)

Bota

(dpm)

$-0.37$

5.22
Comments

20080826154749-A9 
NSTec

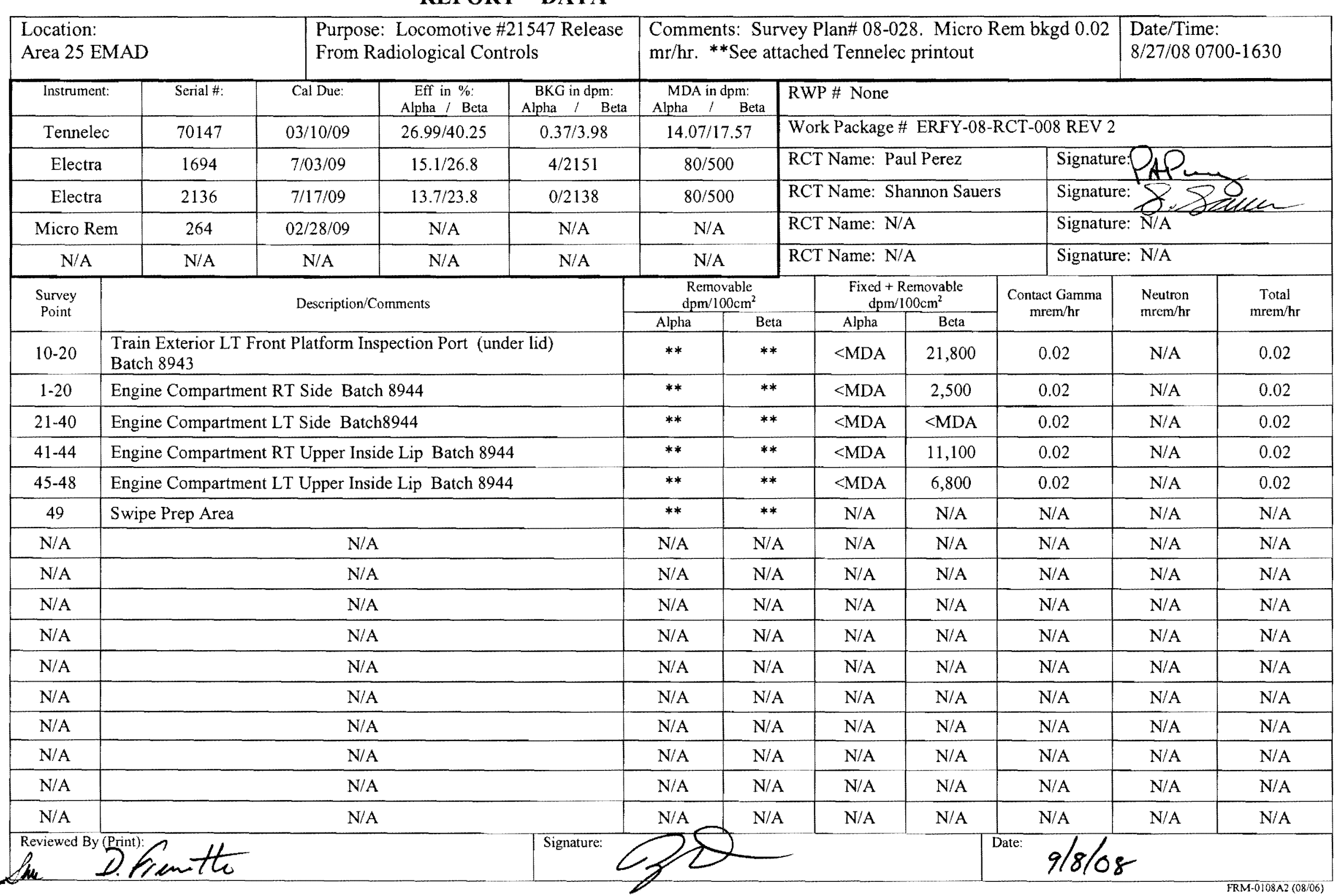

RADIOLOGICAL SURVEY

REPORT - DATA

SURVEY \# 08-ER-A25-185

Page 18 of 23

Date/Time:

\begin{tabular}{l|l}
$\mathrm{mr} / \mathrm{hr},{ }^{* *}$ See attached Tennelec printout & $8 / 27 / 080700-1630$
\end{tabular}

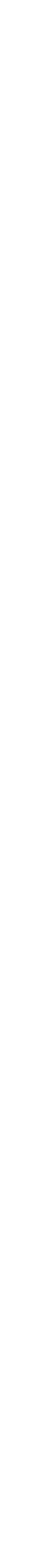


Device: Tennelec 70147

Batch 1D: Swipes - 200808270634

Background (dpm)

Alpha Rate: $\quad 0.37$

Beta Rate:
Efficiency $(\%)$

Alpha: 26.99

Bota: 40.25
MDA (DPM)

Alpha: 14.07

Beta: 17.57
Count Date: $\quad 8 / 27 / 2008$ 6:34:37AM

Sample Count Time:

1.00 Minutes

Results Report: Page 1 of $1 \quad$ Swipes

Batch Key: 8943

Device:Tennelec 70147

Survey \#O8-ER-A25-185

\section{Sample 1D}

20080827063437-A10 20080827063618-A11 20080827063728-A12 20080827063848-A13 20080827064008-A14 20080827064118-A15 20080827064238-A16 20080827064358-A17 20080827064508-A18 20080827064628-A19 20080827064748-A20

\section{Alpha} (dpm)

$-0.37$

$-0.37$

$-0.37$

3.33

$-0.37$

$-0.37$

$-0.37$

$-0.37$

$-0.37$

$-0.37$

$-0.37$
Beta

(dpm)

3.48

0.99

3.48

5.96

0.99

0.99

$-1.49$

0.99

$-1.49$

$-1.49$

$-1.49$
Comments

Exterior LT Platform Inspection Port (10-20) 
Device: Tennelec 70147

Batch ID: Swipes - 200808270726

Background (dpm)

Alpha Rate: $\quad 0.37$

Beta Rate: $\quad 3.98$

Efficiency $(\%)$

Alpha: 26.99

Beta: 40.25
MDA (DPM)

Alpha: $\quad 14.07$

Bota: $\quad 17.57$
Page 20 of 23

Count Date: $\quad$ 8/27/2008 7:26:22AM

Sample Count Time:

1.00 Minutes

Results Report: Page 1 of $1 \quad$ Swipes

Batch Koy: 8944

Device:Tennelec 70147

Survey \#08-ER-A25-185

Sample ID

20080827072622-A1 20080827072923-A2 20080827073043-A3 20080827073203-A4 20080827073323-A5 20080827073433-A6 20080827073553-A7 20080827073703-A8 20080827073823-A9 20080827073943-A10 20080827074103-A11 20080827074213-A12 20080827074333-A13 20080827074453-A14 20080827074603-A15 20080827074723-A16 20080827074843-A17 20080827075003-A18 200808270751 13-A19 20080827075233-A20 20080827075353-A21 20080827075503-A22 20080827075623-A23 20080827075743-A24 20080827075853-A25 20080827080013-A26 20080827080133-A27 20080827080243-A28 20080827080403-A29 20080827080523-A30 20080827080633-A31 20080827080753-A32 20080827080913-A33 20080827081023-A34 20080827081143-A35 20080827081303-A36 20080827081423-A37 20080827081533-A38 20080827081653-A39 20080827081813-A40 20080827081923-A41 20080827082043-A42 20080827082203-A43 20080827082313-A44 20080827082433-A45 20080827082553-A46 20080827082703-A47 20080827082823-A48 20080827082943-A49

\section{Alpha (dpm)}

$-0.37$

$-0.37$

$-0.37$

$-0.37$

$-0.37$

3.33

$-0.37$

$-0.37$

$-0.37$

$-0.37$

$-0.37$

$-0.37$

$-0.37$

$-0.37$

$-0.37$

3.33

$-0.37$

$-0.37$

$-0.37$

$-0.37$

3.33

3.33

$-0.37$

$-0.37$

$-0.37$

3.33

$-0.37$

$-0.37$

3.33

3.33

$-0.37$

$-0.37$

$-0.37$

3.33

$-0.37$

$-0.37$

$-0.37$

$-0.37$

3.33

3.33

$-0.37$

3.33

$-0.37$

$-0.37$

$-0.37$

7.04

$-0.37$

$-0.37$

3.33
Bota

(dpm)

5.96

$-1.49$

0.99

0.99

0.99

0.99

0.99

3.48

$-1.49$

0.99

0.99

$-1.49$

$-3.98$

3.48

3.48

$-1.49$

$-1.49$

5.96

0.99

8.45

$-1.49$

$-1.49$

0.99

5.96

0.99

$-1.49$

3.48

0.99

5.96

10.93

$-1.49$

5.96

3.48

$-1.49$

$-3.98$

0.99

$-1.49$

5.96

8.45

0.99

$-3.98$

$-1.49$

3.48

$-3.98$

0.99

0.99

$-1.49$

$-3.98$

10.93

\section{Comments}

Eng Compartment Rt Side (1-20)

Eng Compartment LT Side (21-40)
RT Upper Insde Lip (41-44)

LT Upper Inside Lip (45-58) 


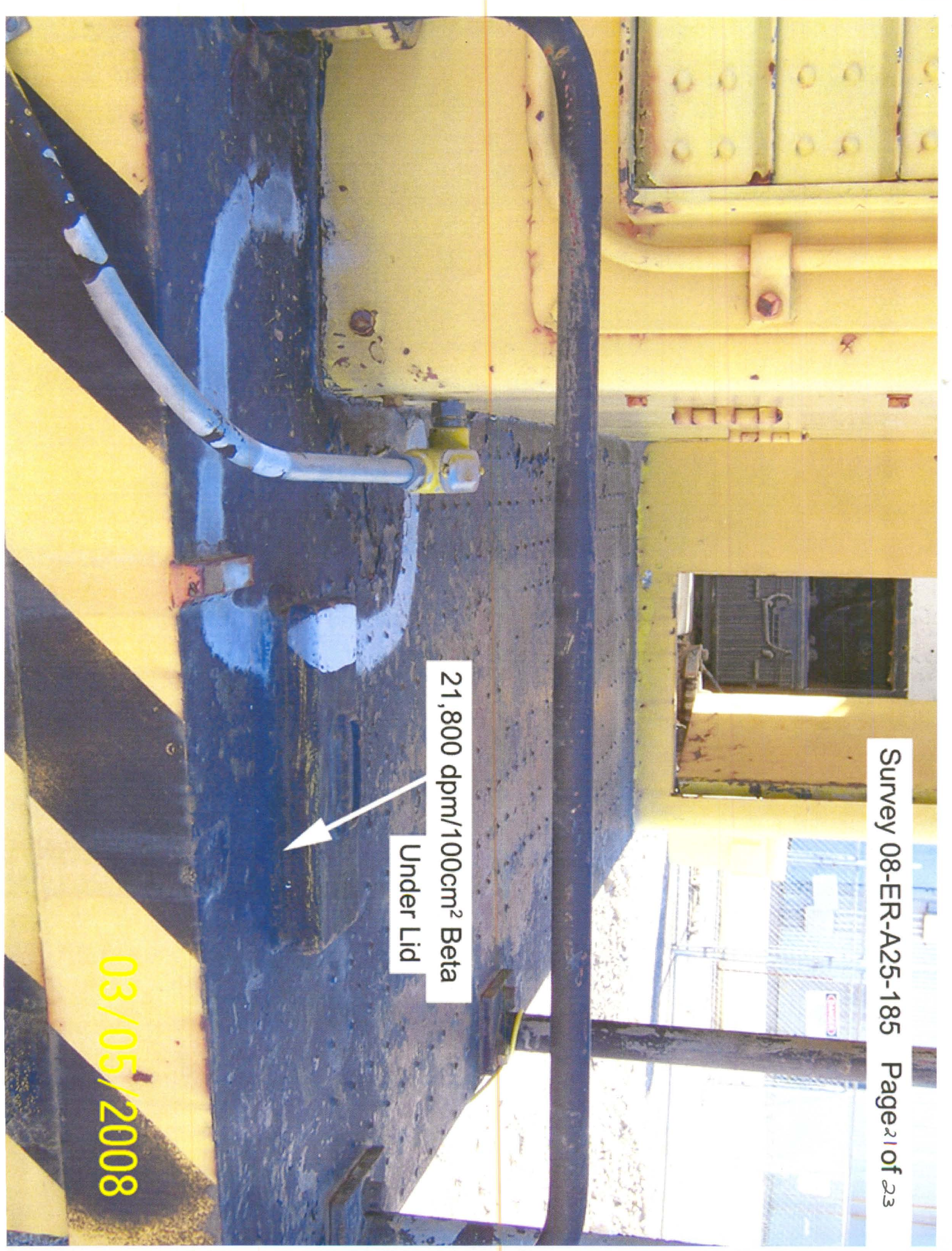




\section{Survey 08-ER-A25-185 Page 22 of 23}

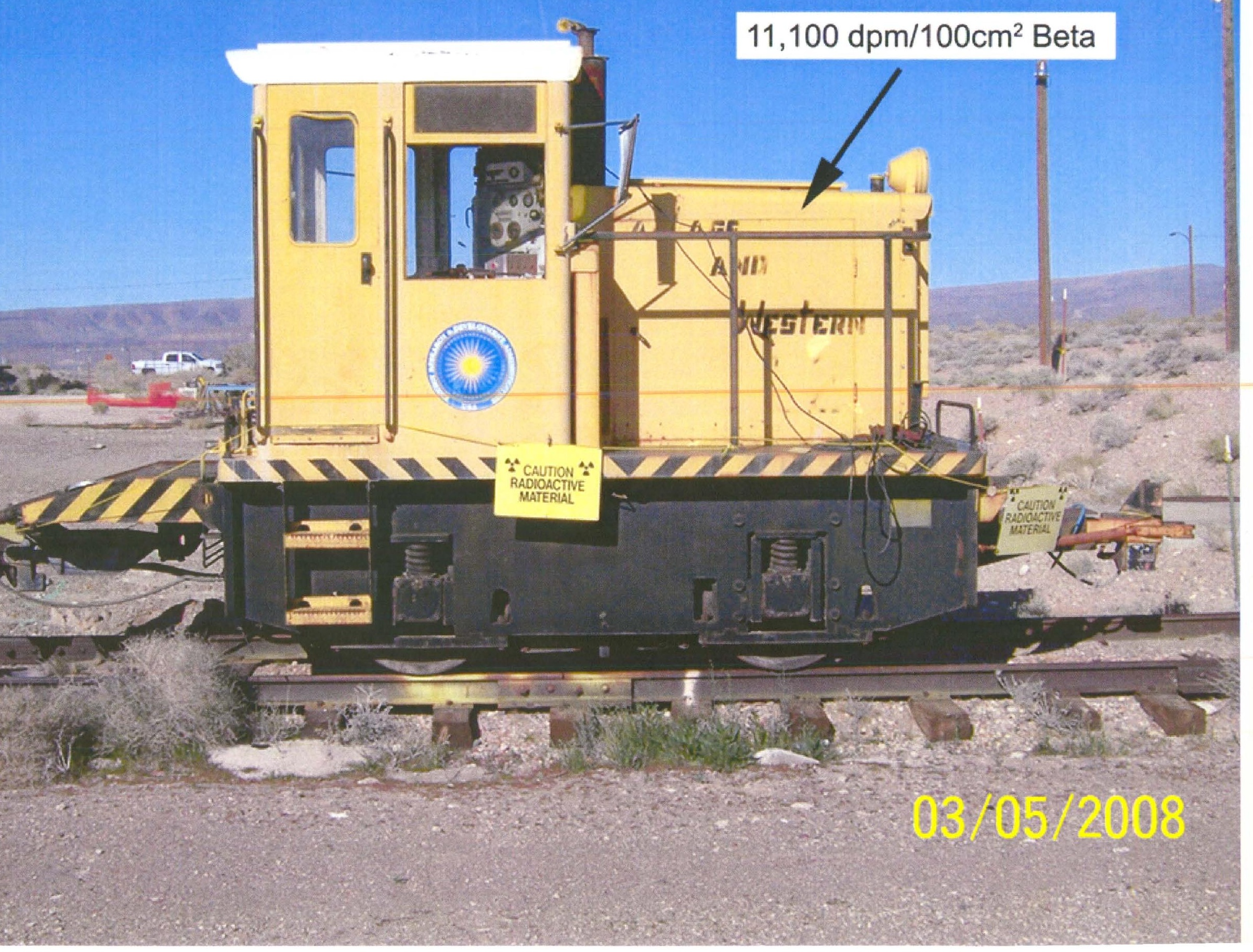




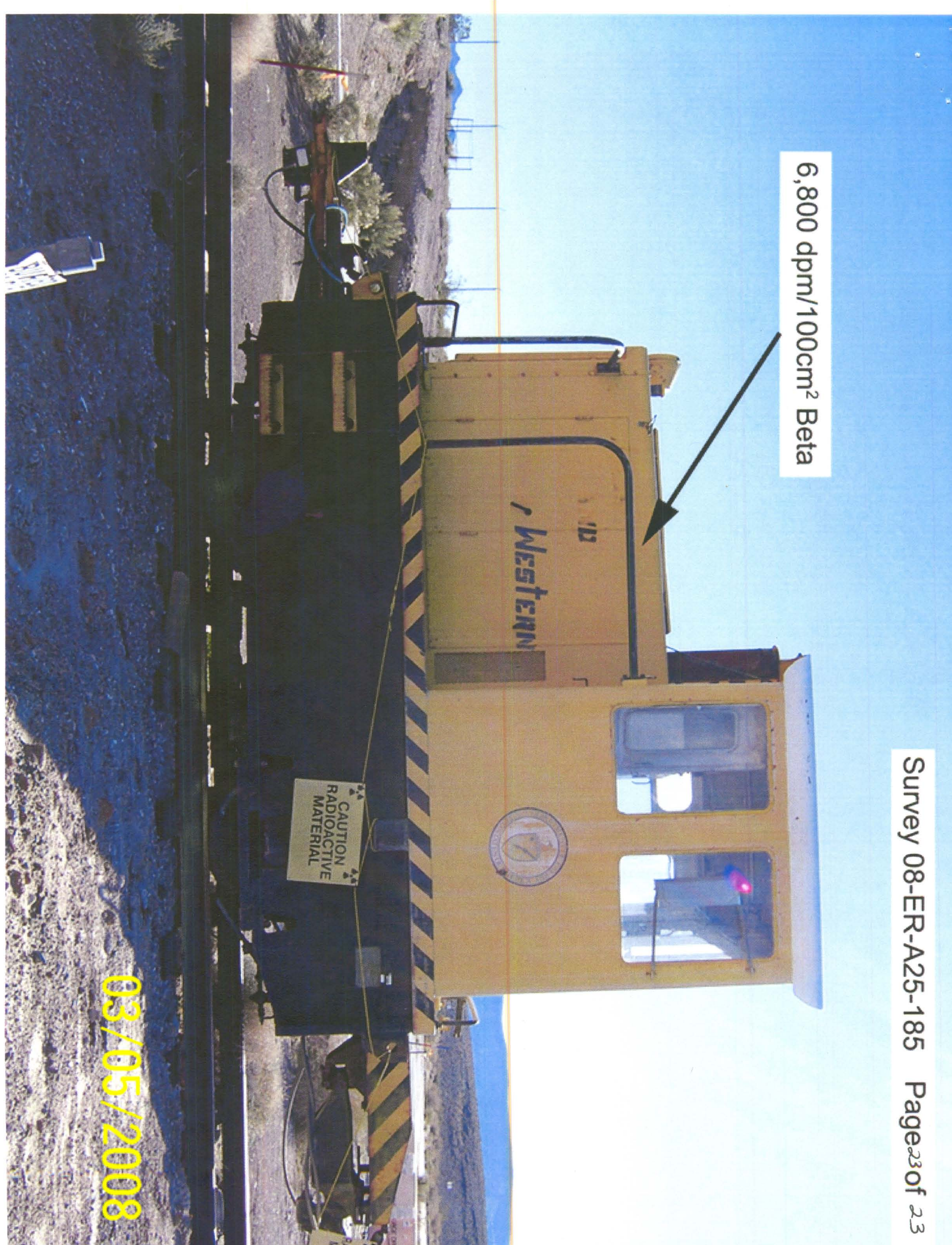


Authorized Limits for Release of the

EM AD 25-Ton Locomotive

May 2010

ATTACHMENT 4

Survey Plan \#09-037, “Post Remediation Survey requirements for the 25

Ton Locomotive at the Engine Maintenance, Assembly and Disassembly (EMAD) Rail Yard Serial Number 21547" 


\section{$\underset{\substack{\text { Nision } \\ \text { Nenvice. Partnership }}}{\text { Saty Technologies } L \text { Lc }}$}

Radiological Operations

Survey Plan

Post Remediation Survey requirements for the 25 Ton Locomotive at the Engine Maintenance, Assembly and Disassembly (EMAD) Rail Yard Serial Number 21547

09-037

January 14, 2010
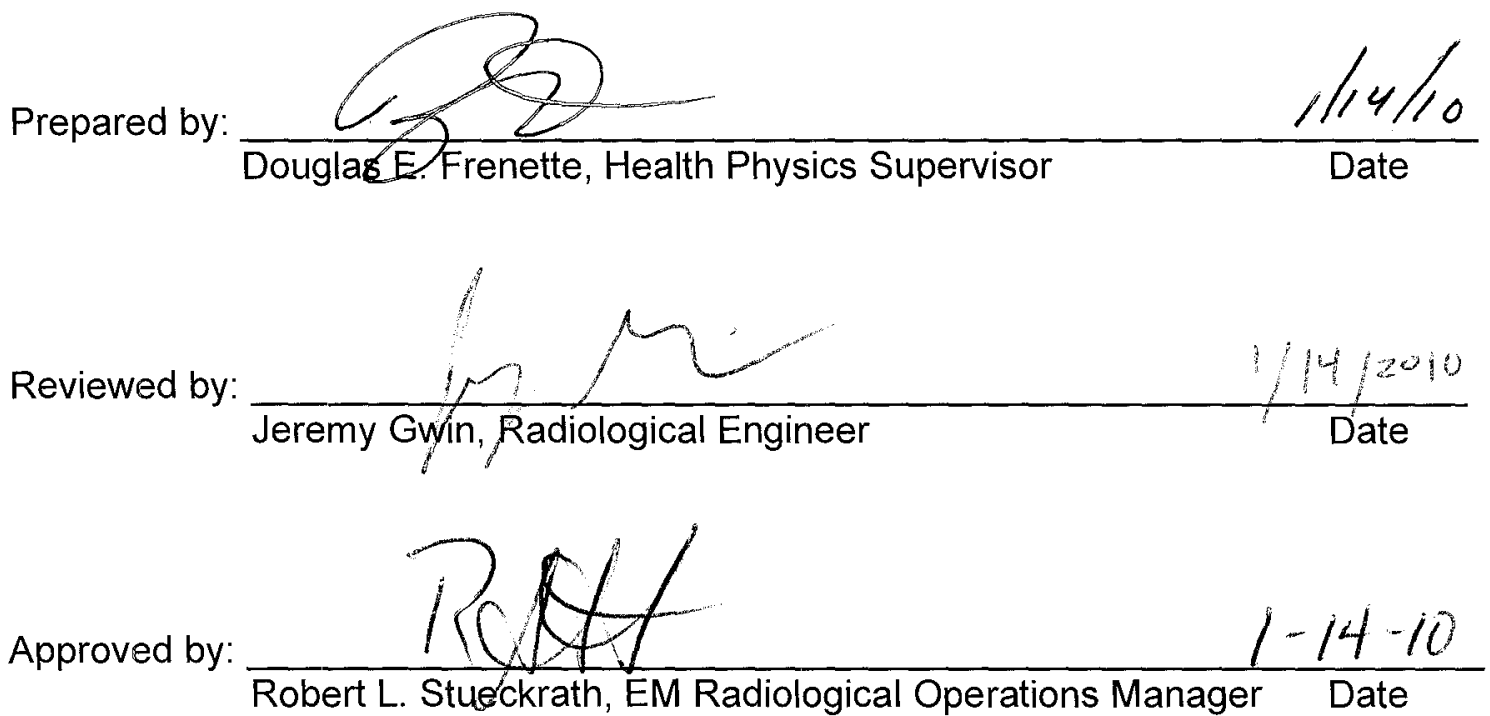


\section{Purpose}

This plan is to provide Radiological Control Technicians (RCTs) with the survey criteria required to provide an unrestricted release of the 25 Ton Locomotive, Serial Number 21547.

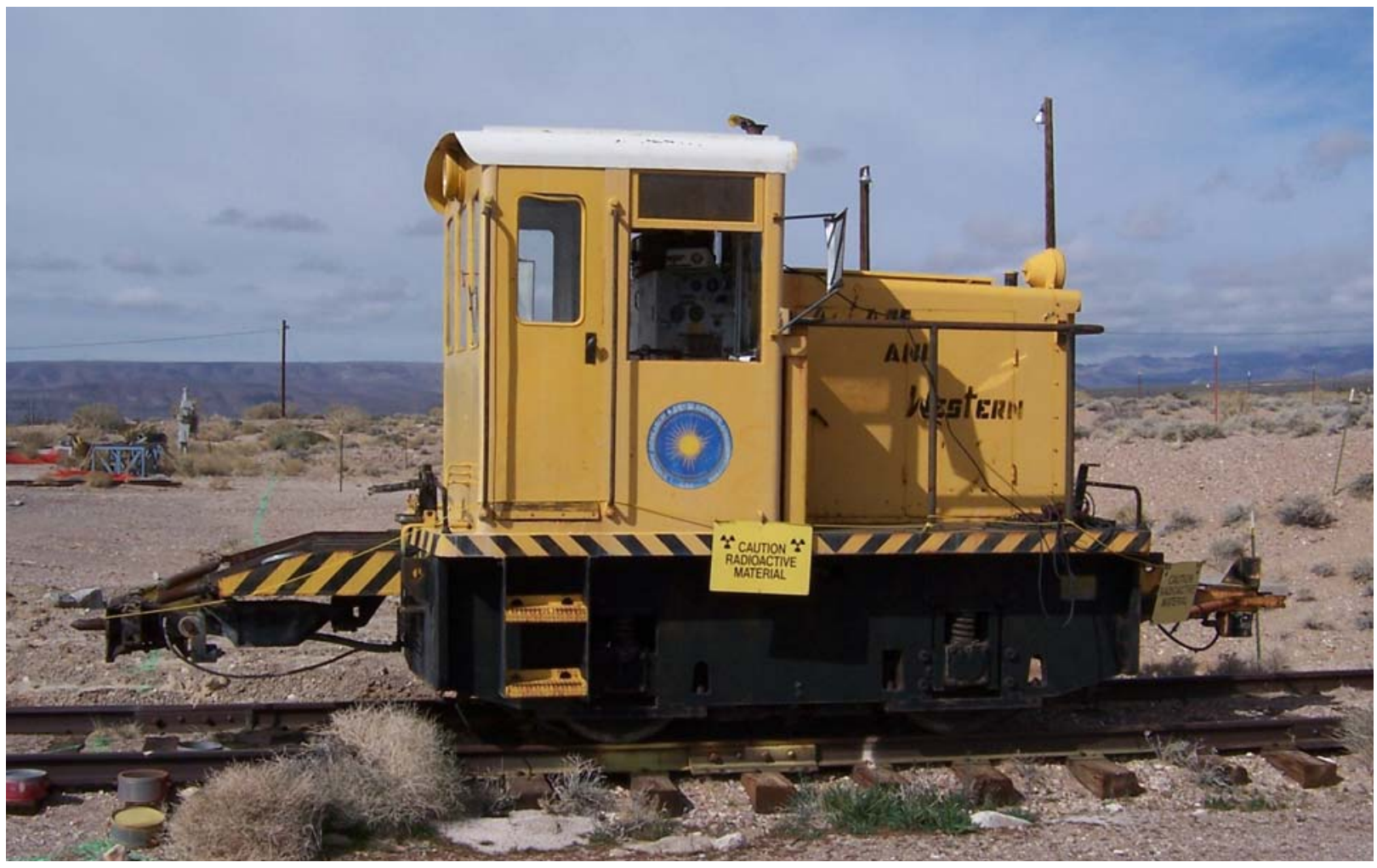

\section{Scope}

This plan pertains to the small 25 Ton Locomotive, Serial Number 21547, located in the Area 25 Engine Maintenance, Assembly and Disassembly (EMAD) rail yard.

\section{History}

The small locomotive was used to move railcars in support of the nuclear rocket/jet engine programs. It is listed at 25 tons and has not been enhanced with added shielding. It is a diesel/electric locomotive with an electric motor mounted in the undercarriage. The locomotive utilizes a drive chain to link the motor to the drive wheels.

Testimony states that it was used to move flat and test cars into position and not for test articles or contaminated equipment. The low traction and poor braking made it undesirable for that type of work. However, there is photographic record of the locomotive being directly coupled to one of the test engines and some of the rails it operated on are known to have been contaminated. 
It also was used at all of the Area 25 test facilities in support of the rocket and jet engine programs. The engine was fully operational at the time that EMAD was shut down and was used to move railcars within the compound after facility closure. It is not known when it was last operated.

Recently, it was posted as a Contamination Area and as a Radioactive Material Area. The reasons for these postings are not really known, although there is testimony that a small container of radioactive material, picked up from along the railroad tracks, was temporarily stored in the cab of the locomotive. The container is no longer in the cab and to the best knowledge available no longer on the EMAD Site. The contamination area signs were removed, but it is not known by whom or by what process.

In April 2008, RCTs performing a casual survey identified three locations on the locomotive that had direct reading contamination in excess of the release limits.

In August 2008, after the development of Survey Plan 08-028, "EMAD Locomotive Survey to Determine Release from Radiological Controls Criteria," RCTs again performed additional surveys of the locomotive and were documented on Survey Report 08-ER-A25-185.

Following the requirements of Survey Plan 08-028, this second survey was much more detailed and comprehensive. Based on the surveys conducted from Survey Plan 08-028, the following areas were found to be impacted:

- Undercarriage Front Brake Assembly - 5,000 dpm/100 $\mathrm{cm}^{2}$ beta

- Undercarriage Front Axle and Wheel - 7,200 dpm/100 $\mathrm{cm}^{2}$ beta

- Front Coupler - 7,000 dpm/100 $\mathrm{cm}^{2}$ beta

- Exterior Right Front Inspection Port - $10,800 \mathrm{dpm} / 100 \mathrm{~cm}^{2}$ beta

- Exterior Left Front Inspection Port - $21,800 \mathrm{dpm} / 100 \mathrm{~cm}^{2}$ beta

- Engine Compartment Right Upper Inside Lip - 11,100 dpm/100 $\mathrm{cm}^{2}$ beta

- Engine Compartment Left Upper Inside Lip - 6,800 dpm/100 $\mathrm{cm}^{2}$ beta

With the discovery of the multiple areas being impacted, the intended disposition of the train to an offsite museum was postponed until a path forward could be determined.

\section{Instructions}

All surveys shall be performed by qualified personnel. Personnel must use their experience, knowledge of the area in which they are working, and workplace indicators to ensure adequate surveys are being performed, in addition to the information provided in this document.

Background levels for direct surveys shall be less than $4,000 \mathrm{dpm} / 100 \mathrm{~cm}^{2}$ beta using the NE Electra.

Based on process knowledge and sufficient characterization data, surveys will consist of measurements for Beta contamination only. All surveys shall be performed and documented in accordance with Organization Instruction OI-0441.211, "Direct and Indirect Surveys." Survey results shall be recorded on a form FRM-0108, "Radiological Survey Report," and approved by a Health Physics Supervisor or designee. 
During this survey process, if contamination is found that is above the suspension limits of the Radiological Work Permit, work shall be paused, personnel will immediately stop all decontamination efforts and follow the RCT's instructions. RCTs will immediately notify the Environmental Restoration Health Physics Supervisor and survey any personnel who were in the affected room or area. Based on the levels encountered, RCTs will also adjust radiological postings to be commensurate with actual area conditions.

\section{Contamination Surveys}

NSTec Environmental Restoration has been tasked with decontaminating the 25 Ton Locomotive at the Area 25 EMAD rail yard. The decontamination performed will be to the extent and rigor required to reduce the level of contamination on the seven impacted areas to meet the requirements of DOE/NV/25946--801, "Nevada Test Site Radiological Control Manual," Table 4-2. Successful completion of this activity will be documented on the pre and post decontamination surveys of each of the seven impacted locations.

RCTs will survey and document a pre decontamination survey and post decontamination survey for each of the following locations:

1. Undercarriage Front Brake Assembly

2. Undercarriage Front Axle and Wheel

3. Front Coupler

4. Exterior Right Front Inspection Port

5. Exterior Left Front Inspection Port

6. Engine Compartment Right Upper Inside Lip

7. Engine Compartment Left Upper Inside Lip

Note: A copy of Survey Report 08-ER-A25-185 will provide exact locations of impacted areas.

When performing decontamination, extreme care should be given to prevent the spread of contamination to additional areas on the locomotive. Post decontamination surveys shall include surveying a small radius ( $\sim$ ") immediately adjacent to the remediated area. In some cases, this may not be feasible due to physical constraints of the area being remediated so the RCT should use their experience and knowledge to evaluate any surface area that could have potentially been cross contaminated during the decontamination process.

To the extent practical, RCTs shall collect the decontamination byproduct for isotopic validation using gamma spectroscopy. Gamma spectroscopy shall be performed by qualified personnel and the results reviewed and approved by Radiological Engineering.

\section{Disposition}

This survey plan is only to demonstrate the successful completion of decontamination of the known impacted areas of the locomotive. There is no intended disposition in regards to the criteria set forth in this survey plan. Disposition may be evaluated and criteria set forth in future survey plans and documentation. 
Authorized Limits for Release of the

EM AD 25-Ton Locomotive

May 2010

\section{ATTACHMENT 5 \\ Survey Results \#10-ER-A25-097 for Survey Plan \#09-037}


NSTec

\section{RADIOLOGICAL SURVEY REPORT - DATA}

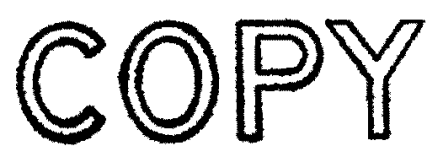

Location Purpose:

Post Decon of Locomotive \# 21547
SURVEY \# 10-ER-A25-097

Page 1 of 2

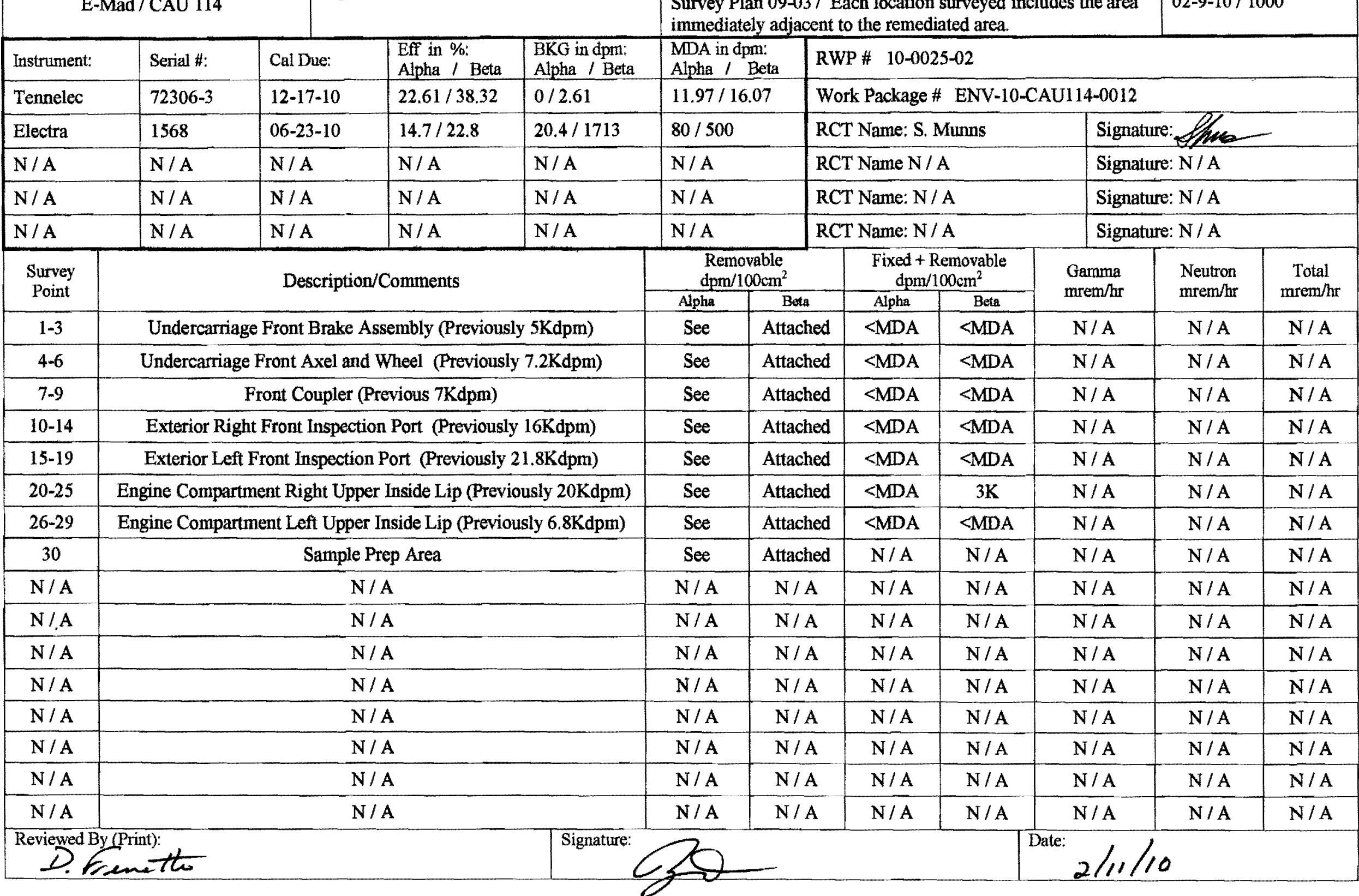


Device: $72306-3$

Page 2 of 2

Batch ID: Swipes - 201002091142

Count Date: $\quad$ 2/9/2010 11:42:58AM

Background (dpm) Efficiency (\%) MDA (DPM)

Alpha Rate: $\quad 0.00$

Alpha: 22.61

2.61

Beta: 38.32

Alpha: $\quad 11.97$

Beta: 16.07

Sample Count Time:

1.00 Minutes

Results Report: Page 1 of 1

Swipes

Batch Key: 1065

Device:72306-3

Survey \# 10-ER-A25-097

\section{Sample ID}

0100209114258-A1

0100209114429-A2

$0100209114539-A 3$

0100209114649-A4

0100209114809-A5

0100209114919-A6

0100209115029-A7

0100209115139-A8

0100209115259-A9

0100209115409-A10

0100209115519-A11

0100209115629-A12

0100209115749-A13

0100209115859-A14

0100209120009-A15

J100209120119-A16

J100209120229-A17

3100209120349-A18

J100209120459-A19

2100209120609-A20

3100209120719-A21

3100209120839-A22

$3100209120949-A 23$

3100209121059-A24

$3100209121209-A 25$

J100209121329-A26

3100209121439-A27

$1100209121549-A 28$

$1100209121659-A 29$

)100209121809-A30

\section{Alpha \\ (dpm)}

0

0

0

0

0

0

0

4.42

0

0

0

0

4.42

0

0

0

0

0

4.42

0

0

4.42

0

0

0

4.42

0

4.42

0

0
Beta

(dpm)

7.83

5.22

0.00

0.00

10.44

$-2.61$

2.61

2.61

2.61

2.61

0.00

$-2.61$

$-2.61$

2.61

5.22

7.83

5.22

$-2.61$

0.00

5.22

0.00

0.00

2.61

0.00

7.83

0.00

13.05

$-2.61$

5.22

0.00

\section{Comments}

Undercarriage Front Brake Assy<smiles>[Te]=[Te]</smiles>

Undercarriage From Axel and Wheel

Front Coupler

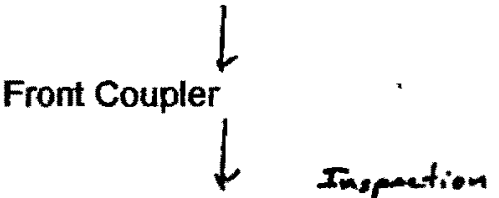

Exterior Right Front Inspeotein Port

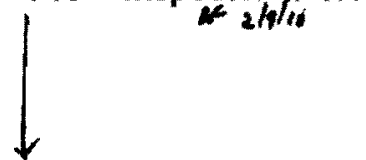

Exterior Left Front Inspection Port<smiles>CCCC</smiles>

Engine Compartment Right Upper Inside Lip

Engine Compartment Left Upper Inside Lip

Sample Prep Area 
Authorized Limits for Release of the

EM AD 25-Ton Locomotive

May 2010

ATTACHMENT 6

REC-2010-001, "Public Dose Estimate from the EMAD 25 Ton Locomotive" 


\title{
National Security Technologies LLc
}

\section{Radiological Engineering}

Calculation

\author{
Environmental Restoration
}

\section{PUBLIC DOSE ESTIMATE FROM THE EMAD 25 TON LOCOMOTIVE}

\section{REC-2010-001}

Revision 1
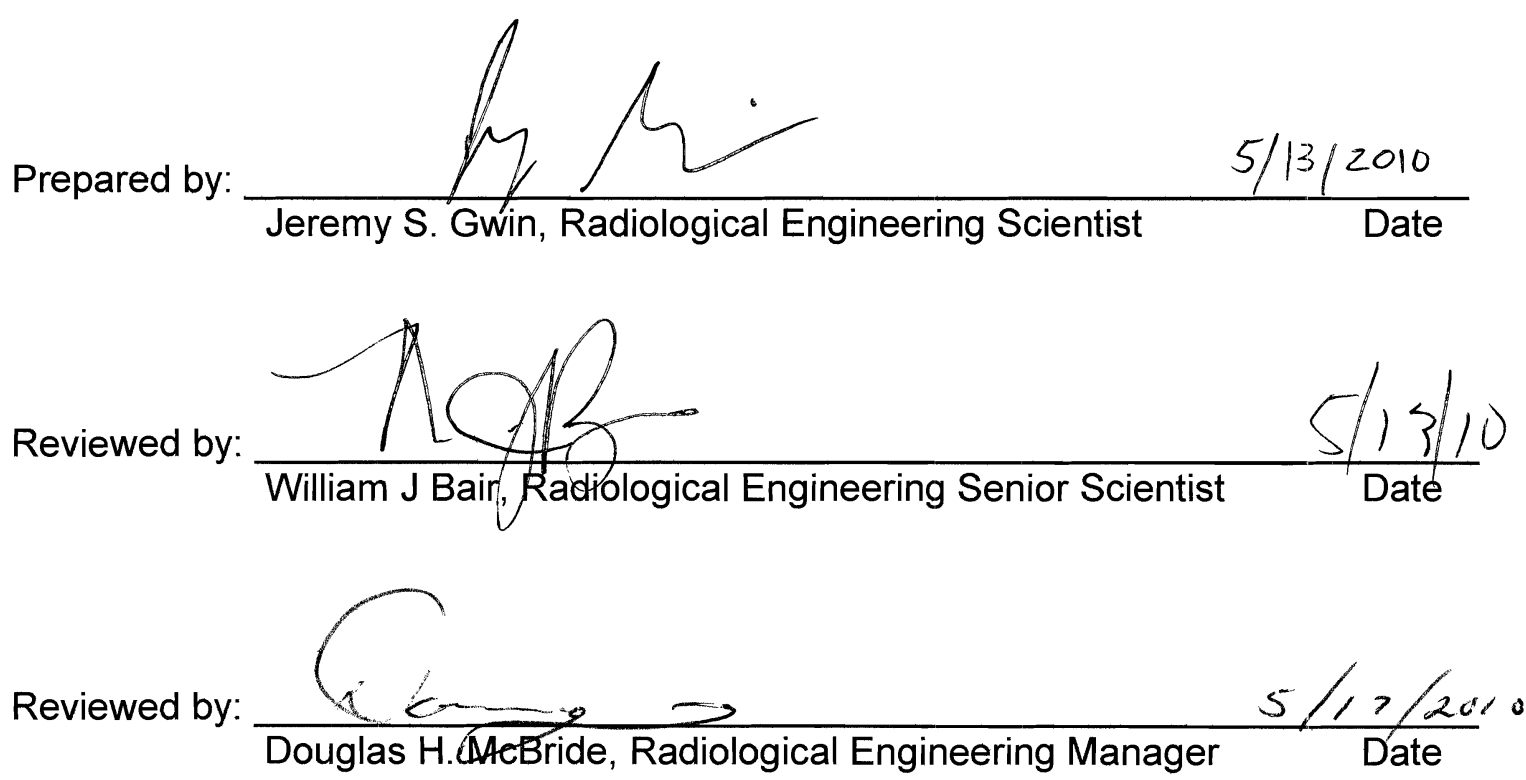


\section{INTRODUCTION}

\subsection{Purpose}

This National Security Technologies, LLC (NSTec) Radiological Engineering Calculation is to estimate the radiation dose originating from residual radioactivity within a small 25 ton locomotive, Serial Number 21547, currently located at the Engine Maintenance Assembly and Disassembly (EMAD) rail yard in Area 25 of the Nevada Test Site (NTS) pictured in Figure 1. This locomotive is planned for unrestricted release to the Nevada State Railroad Museum in Boulder City, NV where it will be used as a display piece. The dose will be calculated using the U.S. Department of Energy (DOE) approved modeling code RESRAD-BUILD v3.5.

Figure 1. EMAD 25 Ton Locomotive

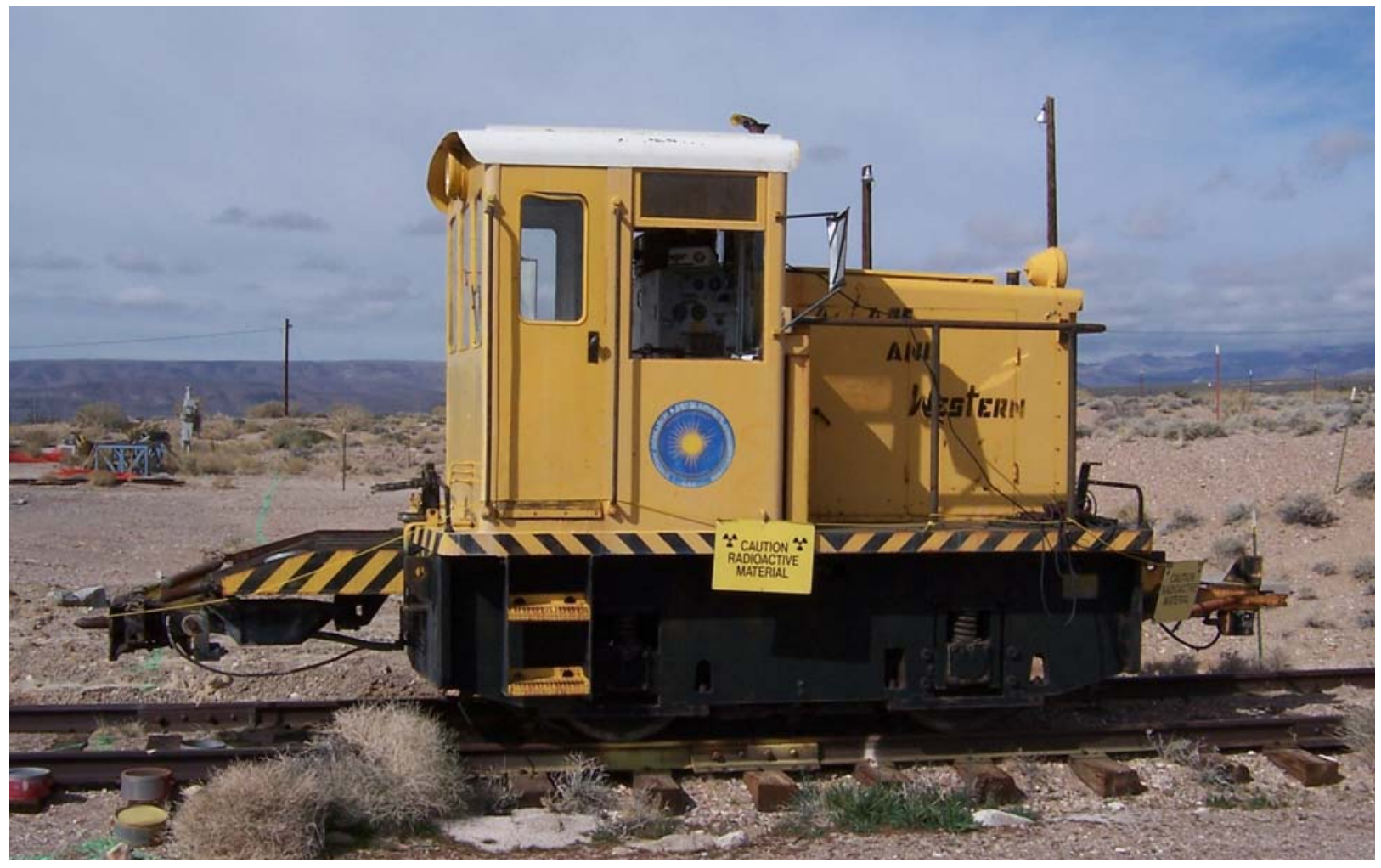




\subsection{History}

The small locomotive was used to move railcars in support of the nuclear rocket and jet engine programs. It is listed at 25 tons and has not been enhanced with added shielding. It is a diesel/electric locomotive with an electric motor mounted in the undercarriage. The locomotive utilizes a drive chain to link the motor to the drive wheels.

Testimony states that it was used to move flat and test cars into position and not for test articles or contaminated equipment. The low traction and poor braking made it undesirable for that type of work. However, there is a photographic record of the locomotive being directly coupled to one of the test engines. In addition, some of the rails it operated on are known to have been contaminated.

The locomotive was used at all of the Area 25 test facilities in support of the rocket and jet engine programs. The engine was fully operational at the time that EMAD was shut down and was used to move railcars within the compound after facility closure. It is not known when it last operated.

\subsection{Radiological Conditions}

In the past, the locomotive was posted as a contamination area. The reasons for these postings are not known, although there was testimony that a small container of radioactive material, picked up from along the railroad tracks, was temporarily stored in the cab of the locomotive. The container is no longer in the cab and to the best knowledge available no longer at the EMAD Site. The contamination area signs were removed, but it is not known by whom or by what process. Currently, the locomotive is posted as a radioactive material area.

In April 2008, Radiological Control Technicians (RCTs) performing a casual survey identified three locations on the locomotive that had contamination in excess of the release limits.

In August 2008, after the development of Survey Plan 08-028, "EMAD Locomotive Survey to Determine Release from Radiological Controls Criteria," RCTs performed an additional survey of the locomotive which was documented on Survey Report Number 08-ER-A25-185. Following the requirements of Survey Plan 08-028, this survey was much more detailed and comprehensive. Based on the survey conducted from Survey Plan 08-028, the following areas were found to be impacted:

- Undercarriage Front Brake Assembly - 5,000 dpm/100 $\mathrm{cm}^{2}$ total beta

- Undercarriage Front Axle and Wheel - 7,200 dpm/100 $\mathrm{cm}^{2}$ total beta

- Front Coupler - 7,000 dpm/100 $\mathrm{cm}^{2}$ total beta

- Exterior Right Front Inspection Port - $10,800 \mathrm{dpm} / 100 \mathrm{~cm}^{2}$ total beta

- Exterior Left Front Inspection Port - 21,800 dpm/100 $\mathrm{cm}^{2}$ total beta

- Engine Compartment Right Upper Inside Lip - 11,100 dpm/100 $\mathrm{cm}^{2}$ total beta

- Engine Compartment Left Upper Inside Lip - 6,800 dpm/100 $\mathrm{cm}^{2}$ total beta 
No removable contamination was found. The locations of the elevated fixed contamination are not immediately adjacent to any of the inaccessible areas of the locomotive. These inaccessible areas were inaccessible for the portable survey meter. Inaccessible areas were surveyed for removable contamination to some extent (swipes), except for the interior portions of the engine and other sealed components. This provided a reasonable assurance that contamination did not migrate to the inaccessible surfaces since the pathways were not contaminated and the locations with elevated contamination were remote to these pathways.

With the discovery of the multiple areas being impacted, the intended disposition of the locomotive to an offsite museum was postponed until a path forward for unrestricted release could be determined.

As part of the path forward for unrestricted release, the identified areas of known fixed contamination on the locomotive were decontaminated in January-February 2010 under Survey Plan 09-037, "Post Remediation Survey Requirements for the 25 Ton Locomotive at the Engine Maintenance, Assembly and Disassembly (EMAD) Rail Yard Serial Number 21547." This decontamination process effectively removed the areas of known contamination on the surfaces of the locomotive to less than detectable limits of the survey instrument, with the exception of one location. The right upper inside lip of the engine compartment was decontaminated to a beta activity of $3000 \mathrm{dpm} / 100 \mathrm{~cm}^{2}$. The area of the fixed contamination was less than one square meter.

A sample of decontamination effluent and trash was given to Radiological Engineering to perform an isotopic identification. Performing gamma spectroscopy, the isotopes of Cs-137 and U-235 were identified. It was assumed that the beta activity is comprised of a mixture of fission products (Cs-137 and Sr/Y-90). The U-235 was identified by gamma spectroscopy, but is present on the locomotive at or below the detectable limits for portable survey instruments.

\subsection{Requirements for DOE Authorized Release}

The unrestricted release of the locomotive will be based on alternate As Low As Reasonably Achievable (ALARA)/dose-based derived authorized limits in accordance with DOE Order 5400.5, "Radiation Protection of the Public and the Environment." Since it is impossible to prove that there is no residual contamination in inaccessible areas, it will be necessary to demonstrate that the estimated dose to the public meet the following requirements:

- Authorized limits for release of the item must ensure that doses to the public from all sources are less than the primary dose limit for all sources (100 mrem/year).

- Authorized limits for release of the item must be developed and approved by DOE consistent with the ALARA process. These limits will be based on a documented finding that they are as low as practicable as determined through the ALARA process, with a goal of maintaining individual doses low in comparison to background (e.g., a few mrem/year or less). In any case, the limits must be a fraction of the primary dose limit for the public (e.g., 25 mrem/year or less). 
The DOE dose constraint of $25 \mathrm{mrem} / \mathrm{year}$ is also consistent with the dose requirements in Title 10 Code of Federal Regulations (CFR) Part 20, "Standards for Protection Against Radiation," Subpart E, "Radiological Criteria for License Termination." The 25 mrem/year dose constraint is also consistent with the Nevada Administrative Code (NAC) 459.3178, "Property of decommissioned facility: Eligibility for release for unrestricted use." A basic dose assessment using the RESRAD-BUILD v3.5 code should be adequate to demonstrate compliance with the dose constraint of 25 mrem/year.

Per NTS Organization Instruction OI-0441.212 Revision 4, "Controlled and Unrestricted Release," items that are to be released offsite and have inaccessible surfaces and have known contamination or the potential for internal contamination require the approval of the Radiological Control Manager, the senior line manager of the project, and the DOE/NNSA line manager.

\section{DOSE ASSESSMENT INFORMATION}

\subsection{Computer Model: RESRAD-BUILD}

The RESRAD-BUILD computer code is a pathway analysis model developed to evaluate the potential radiological dose incurred by an individual who works or lives in a building contaminated with radioactive material. The radioactive material in the building structure can be released into the indoor air by mechanisms such as diffusion (radon gas and tritiated water), mechanical removal (decontamination activities), or erosion (removable surface contamination). The transport of radioactive material within the building from one compartment to another is calculated with an indoor air quality model. The air quality model evaluates the transport of radioactive dust particulates and radon progeny due to (1) air exchange between compartments and with outdoor air, (2) the deposition and resuspension of particulates, and (3) radioactive decay and ingrowth. RESRADBUILD can model up to three compartments in a building, thereby making it possible to evaluate situations ranging from a one-room warehouse to a three-story house, for example (Argonne National Laboratory, 2003).

As mentioned before, RESRAD-BUILD v3.5 was the code used to model dose from residual radioactivity. This code was chosen because it was the best fit for this type of dose calculation. Although the code is used for residual radioactivity in buildings, the locomotive can be visualized as a radioactive source within a building (a warehouse). As with all dose models, many assumptions were made. A discussion of scenarios and assumptions will now be addressed.

\subsection{Scenarios}

Per telephone conversation, the Museum Director of the Nevada State Railroad Museum in Boulder City, NV informed NSTec Radiological Engineering that the locomotive would be for public display only. This locomotive will not be operational; it will just be used as a static display for the public to view. Current rules at the rail yard prohibit the public from climbing on any of the displays. The rail yard has a fence around the train displays and is locked after business hours. The only foreseeable direct contact with the locomotive is from museum workers, if they have to move it or refurbish it. Although, the director commented that this locomotive looked good and did not need refurbishing, after decontamination, it may need some touch up work on the exterior. 
Four use scenarios were developed from this information and incorporated into the dose modeling of the EMAD locomotive.

- Likely Use Scenario 1: The dose to an individual under a likely use scenario was modeled as a member of the public, the "museum enthusiast," who attends the museum once a week for two hours. The whole two hours were spent 0.3 meters away from the side of the locomotive. For conservatism, the entire side of the locomotive was assumed to be uniformly contaminated with the maximum activity that was found on previous surveys.

- Likely Use Scenario 2: Another likely use scenario involved a "museum worker" who refurbishes or moves the locomotive for 10 hours a day for 30 days. The time was spent 0.3 meters away from the sides of the locomotive, partially surrounded by a contaminated engine compartment.

- Worst Case Scenario 1: A dose under worst plausible use scenarios, or "worst case" scenario, would represent a situation if all controls failed. A scenario was hypothesized and involved an individual spending an unreasonable amount of time in close proximity to a contaminated engine compartment. This person would spend eight hours a day, every day, at 0.3 meters away from the side of the "contaminated" compartment.

- Worst Case Scenario 2: Another worst case scenario involved a museum employee who mechanically removes (by grinding or sanding) the small area of fixed contamination which remains on the locomotive. This scenario assumed that the total amount of known contamination becomes airborne, that the employee is 0.3 meters away, and spends six hours performing work.

\subsection{Assumptions}

With RESRAD-BUILD, the locomotive was modeled as an area source, or multiple area sources. The source activity was assumed as the highest fixed contamination found on the exterior of the locomotive $\left(22,000 \mathrm{dpm} / 100 \mathrm{~cm}^{2}\right.$ total beta) and was assumed to consist of the isotopes $\mathrm{Sr} / \mathrm{Y}-90, \mathrm{Cs}-137$, and $80 \mathrm{dpm} / 100 \mathrm{~cm}^{2}$ of U-235. The entire surface of the locomotive was assumed to have uniform contamination. This was a conservative assumption because all but one of the known areas of fixed contamination on the exterior were decontaminated to values less than the Minimum Detectable Activity for the portable survey instruments used at the NTS $(<80 \mathrm{dpm}$ alpha and $<500$ $\mathrm{dpm}$ beta). The right upper inside lip of the engine compartment was decontaminated to a beta activity of $3000 \mathrm{dpm} / 100 \mathrm{~cm}^{2}$. The area of the fixed contamination spot was less than 1 square meter.

For each scenario, the source in the dose model varied. Some of the assumptions are discussed below.

- For the "Likely Use Scenario 1" model, a $3.66 \times 2.44$ meter $(12 \times 8 \mathrm{ft})$ area source was assumed to contain the maximum fixed contamination underneath $0.4 \mathrm{~cm}$ of steel shielding. The thickness of the shield is representative of the steel, exterior frame of the locomotive. The removable fraction of the source was set at $1 \%$. A $1 \times 1$ meter area source was used to mimic the small area of fixed contamination 
remaining on the train (no shielding used). The removable fraction of this source was set at $10 \%$. The receptor was then positioned 0.3 meters away from the area sources.

- For the "Likely Use Scenario 2" model, the source was actually three, $2 \times 2$ meter area sources positioned in front, on the left, and right side of the worker. This configuration was made to mimic two open engine compartment doors and another surface in close contact with the employee. This would also work for an employee in the cab of the locomotive, with the floor, ceiling, and wall of the engine compartment represented as the three sources. Two of the area sources were assumed to contain the maximum fixed contamination underneath $0.4 \mathrm{~cm}$ of steel shielding. The removable fraction of these sources was set at $1 \%$. The other source was used to mimic the small area of fixed contamination remaining on the train (no shielding used). The removable fraction of the source was set at $10 \%$. The receptor was then positioned 0.3 meters away from the area sources.

- The "Worst Case Scenario 1" model entailed six sources, essentially forming a box. This configuration was used to model the engine compartment. Five of the area sources were assumed to contain the maximum fixed contamination underneath 0.1 $\mathrm{cm}$ of steel shielding. The removable fraction of the sources was set at $10 \%$. The other source was used to mimic the small area of fixed contamination remaining on the train (no shielding used). The removable fraction of this source was set at $10 \%$. The receptor was then positioned 0.3 meters away from the area sources.

- For the "Worst Case Scenario 2" model, a $1 \times 1$ meter area source was used to mimic the small area of fixed contamination remaining on the train (no shielding used). The individual would mechanically remove the total activity without using protective measures. The removable fraction of the source was set at $100 \%$. The respirable fraction of the source was set at $50 \%$.

Though the locomotive will be in an outside environment, the RESRAD-BUILD v3.5 code was appropriate. The assumption that the locomotive resides inside a warehouse was a conservative assumption. The radionuclide resuspension rate and air flow in the building increased the potential for an internal dose compared to having the locomotive exposed to the outside atmosphere. The code only calculates external and internal dose to individuals from a source of radiation.

For a complete list of all input parameters for each scenario used in the RESRAD-BUILD v3.5 code to perform the dose calculation, see Appendix A.

\section{Work}

RESRAD-BUILD v3.5 was used to perform the dose calculations. No hand calculations were performed. 


\section{Results}

The RESRAD-BUILD detailed results for each scenario may be found in Appendix B Appendix $E$. A summary of the doses received for each scenario over time is presented in Table 1.

Table 1

\begin{tabular}{|c|c|c|c|c|}
\hline & \multicolumn{4}{|c|}{ Dose Received for the Exposure Duration (mrem) } \\
\hline Scenario & $\begin{array}{c}\text { End of the } 1^{\text {st }} \\
\text { year }\end{array}$ & $\begin{array}{c}\text { End of the } 2^{\text {nd }} \\
\text { year }\end{array}$ & $\begin{array}{c}\text { End of the 10 } \\
\text { year }\end{array}$ & $\begin{array}{c}\text { End of the } 30^{\text {th }} \\
\text { year }\end{array}$ \\
\hline Likely Use 1 & 0.109 & 0.105 & 0.085 & 0.0536 \\
\hline Likely Use 2 & 0.512 & 0.491 & 0.399 & 0.252 \\
\hline Worst Case 1 & 3.83 & 3.30 & 2.68 & 1.69 \\
\hline Worst Case 2 & 1.16 & 0 & 0 & 0 \\
\hline
\end{tabular}

The "Worst Case Scenario 2" model assumed that $100 \%$ of the source activity was removed in a day (complete mechanical removal of the source activity within six hours). With the source completely removed after six hours, the dose received from that time after was null.

\section{Conclusion}

The "Likely Use Scenario 1" represented members of the public visiting the museum and viewing the locomotive. The "museum enthusiast" could receive a maximum dose of 0.109 mrem in any given year. This scenario had the highest probability of occurring out of the four scenarios. This individual would spend two hours per week, every week for 30 years, standing $30 \mathrm{~cm}$ away from the exterior surface of the locomotive. The expected dose to an individual in this group would be a fraction of the calculated dose because the source term was exaggerated in the RESRAD-BUILD code. The calculated maximum dose under this likely use scenario is well under the $25 \mathrm{mrem} / \mathrm{year}$ dose constraint and meets the criteria of a "few millirem or less in a year" established in DOE O 5400.5 .

The "Likely Use Scenario 2" represented those employees of the museum who need to refurbish or move the locomotive. The "museum worker" could receive a maximum dose of 0.523 mrem in any given year. This scenario was less probable than "Likely Use Scenario 1." The museum employee would spend 10 hours a day, for 30 days a year, positioned partially enclosed between three contaminated surfaces at $30 \mathrm{~cm}$ away from each surface. The expected dose to an individual in this group would be a fraction of the calculated dose because the source term was exaggerated in the RESRAD-BUILD code. The calculated maximum dose under the likely use scenario is well under the 25 mrem/year dose constraint and meets the criteria of a "few millirem or less in a year" established in DOE O 5400.5. 
Under the "Worst Case Scenario 1" scenario, an individual could receive a maximum dose of 3.83 mrem in any given year. Again, the worst case scenario represented the dose to an individual if all controls failed. This individual would spend 8 hours per day, every day for 30 years, in close proximity to a surface contaminated engine compartment. The source activity for the "contaminated" engine compartment was exaggerated, thus increasing the dose to the individual. The probability of this scenario occurring is extremely low. The calculated maximum dose under the worst case scenario is well under the 25 mrem/year dose constraint established in DOE O 5400.5.

Under the "Worst Case Scenario 2," an individual could receive a maximum dose of 1.16 mrem in any given year. Essentially, the individual would receive a one-time $1.16 \mathrm{mrem}$ dose during a six hour exposure. This individual would spend a total of six hours mechanically removing a small surface contaminated area. The source activity for the contaminated area mimicked actual conditions. The airborne respirable fraction was set at $50 \%$, which equates to half of the total source activity becoming airborne and existing as respirable particulate. This was a conservative assumption because most of the source activity would not exist as respirable fractions during mechanical removal (i.e., mechanical removal fragments would be too large to become respirable). The probability of this scenario occurring is low. The calculated maximum dose under this scenario is well under the 25 mrem/year dose constraint established in DOE O 5400.5.

With all four scenarios below the $25 \mathrm{mrem} /$ year dose constraint and meeting the "few millirem in a year" criteria, the EMAD 25 ton locomotive meets radiological requirements to be released with residual radioactivity to the public. 


\section{Appendix A \\ Input Parameters in RESRAD-BUILD for Each Scenario}

\section{List of Assumptions and Parameters for Likely Use Scenario 1}

Any parameter that is not specifically mentioned uses the RESRAD-BUILD default value.

1. Case

- Case

o Dose Risk Library: ICRP 72 (Adult)

- Time Parameters

o Exposure Duration (days): 365

o Indoor fraction: 0.5

- Evaluation Times - Time Integration

o Maximum number of points for Dose/Risk: 17

2. Building Parameters

- Number of Rooms: 1

- Deposition Velocity: $0.01 \mathrm{~m} / \mathrm{s}$

- Resuspension Rate: 0.0000005 (1/s)

- Air Flow

o Building Exchange Rate: $0.8(1 / \mathrm{h})$

o Room 1

- Area $\left(\mathrm{m}^{2}\right): 36$

- Height (m): 2.5

3. Radiological Units

- Activity: dpm

- Dose: mrem

4. Receptor Parameters

- Receptor: 1

- Room: 1

- Time fraction: 0.025

- Breathing Rate: $18 \mathrm{~m}^{3} / \mathrm{d}$

- Ingestion Rate: $0.0001 \mathrm{~m}^{2} / \mathrm{h}$

- Location:

- $\mathrm{X}: 1.3, \mathrm{Y}: 1, \mathrm{Z}: 1$

5. Shielding Parameters

- Source 1 / Receptor 1

o Thickness: $0.4 \mathrm{~cm}$

o Density: $7.6 \mathrm{~g} / \mathrm{cc}$

o Material: Iron

- $\quad$ Source 2 / Receptor 1

o Thickness: $0 \mathrm{~cm}$

o Density: $7.6 \mathrm{~g} / \mathrm{cc}$

o Material: Iron 
6. Source Parameters

- Area sources

- Details (source 1)

o Location: X: 1, Y: 1, Z: 1

o Rectangular Geometry

o Length along (m) Y: 3.66, Z: 2.44

o Release

- Air fraction: 0.1

- Direct Ingestion: 0 1/h

- Removable fraction: 0.01

- Lifetime (d): 365

o Radionuclide: Sr-90 Activity: $1.1 \mathrm{E} 6 \mathrm{dpm} / \mathrm{m}^{2}$

o Radionuclide: Cs-137 Activity: $1.1 \mathrm{E} 6 \mathrm{dpm} / \mathrm{m}^{2}$

o Radionuclide: U-235 Activity: $8.0 \mathrm{E} 3 \mathrm{dpm} / \mathrm{m}^{2}$

- Details (source 2)

o Location: X: 1.6, Y: 1, Z: 1

o Rectangular Geometry

o Length along $(\mathrm{m}) \mathrm{Y}: 1, \mathrm{Z}: 1$

o Release

- Air fraction: 0.1

- Direct Ingestion: 0 1/h

- Removable fraction: 0.1

- Lifetime (d): 365

o Radionuclide: Sr-90 Activity: $1.5 \mathrm{E} 5 \mathrm{dpm} / \mathrm{m}^{2}$

o Radionuclide: Cs-137 Activity: $1.5 \mathrm{E} 5 \mathrm{dpm} / \mathrm{m}^{2}$

o Radionuclide: U-235 Activity: $8.0 \mathrm{E} 3 \mathrm{dpm} / \mathrm{m}^{2}$

\section{List of Assumptions and Parameters for Likely Use Scenario 2}

Any parameter that is not specifically mentioned uses the RESRAD-BUILD default value.

1. Case

- Case

o Dose Risk Library: ICRP 72 (Adult)

- Time Parameters

o Exposure Duration (days): 365

o Indoor fraction: 0.5

- Evaluation Times - Time Integration

o Maximum number of points for Dose/Risk: 17

2. Building Parameters

- Number of Rooms: 1

- Deposition Velocity: $0.01 \mathrm{~m} / \mathrm{s}$

- Resuspension Rate: 0.0000005 (1/s)

- Air Flow

o Building Exchange Rate: 0.8 (1/h)

o Room 1

- Area $\left(\mathrm{m}^{2}\right): 36$

- Height (m): 2.5 
3. Radiological Units

- Activity: dpm

- Dose: mrem

4. Receptor Parameters

- Receptor: 1

- Room: 1

- Time fraction: 0.069

- Breathing Rate: $18 \mathrm{~m}^{3} / \mathrm{d}$

- Ingestion Rate: $0.0001 \mathrm{~m}^{2} / \mathrm{h}$

- Location:

- $\mathrm{X}: 1, \mathrm{Y}: 1, \mathrm{Z}: 1$

5. Shielding Parameters

- Source 1 / Receptor 1

o Thickness: $0.4 \mathrm{~cm}$

o Density: $7.6 \mathrm{~g} / \mathrm{cc}$

o Material: Iron

- $\quad$ Source 2 / Receptor 1

o Thickness: $0.4 \mathrm{~cm}$

o Density: $7.6 \mathrm{~g} / \mathrm{cc}$

o Material: Iron

- $\quad$ Source 3 / Receptor 1

o Thickness: $0 \mathrm{~cm}$

o Density: $7.6 \mathrm{~g} / \mathrm{cc}$

o Material: Iron

6. Source Parameters

- Area sources

- Details (source 1 and 2)

o Location Source 1: X: 0.7, Y: 1, Z: 1

o Location Source 1: X: 1.3, Y: 1, Z: 1

- Rectangular Geometry

o Length along $(\mathrm{m}) \mathrm{Y}: 2, \mathrm{Z}: 2$

o Release

- Air fraction: 0.1

- Direct Ingestion: 0 1/h

- Removable fraction: 0.01

- Lifetime (d): 365

o Radionuclide: Sr-90 Activity: $1.1 \mathrm{E} 6 \mathrm{dpm} / \mathrm{m}^{2}$

o Radionuclide: Cs-137 Activity: $1.1 \mathrm{E} 6 \mathrm{dpm} / \mathrm{m}^{2}$

o Radionuclide: U-235 Activity: $8.0 \mathrm{E} 3 \mathrm{dpm} / \mathrm{m}^{2}$

- Details (source 3)

o Location Source 1: X: 1, Y: 1.3, Z: 1

o Rectangular Geometry

o Length along $(\mathrm{m}) \mathrm{X}: 2, \mathrm{Z}: 2$

o Release

- Air fraction: 0.1

- Direct Ingestion: 0 1/h

- Removable fraction: 0.1

- Lifetime (d): 365 
o Radionuclide: Sr-90 Activity: $1.5 \mathrm{E} 5 \mathrm{dpm} / \mathrm{m}^{2}$

o Radionuclide: Cs-137 Activity: $1.5 \mathrm{E} 5 \mathrm{dpm} / \mathrm{m}^{2}$

o Radionuclide: U-235 Activity: $8.0 \mathrm{E} 3 \mathrm{dpm} / \mathrm{m}^{2}$

\section{List of Assumptions and Parameters for Worst Case Scenario 1}

Any parameter that is not specifically mentioned uses the RESRAD-BUILD default value.

1. Case

- Case

o Dose Risk Library: ICRP 72 (Adult)

- Time Parameters

o Exposure Duration (days): 365

o Indoor fraction: 0.5

- Evaluation Times - Time Integration

o Maximum number of points for Dose/Risk: 17

2. Building Parameters

- Number of Rooms: 1

- Deposition Velocity: $0.01 \mathrm{~m} / \mathrm{s}$

- Resuspension Rate: 0.0000005 (1/s)

- Air Flow

o Building Exchange Rate: 0.8 (1/h)

o Room 1

- Area $\left(\mathrm{m}^{2}\right): 36$

- Height $(\mathrm{m}): 2.5$

3. Radiological Units

- Activity: dpm

- Dose: mrem

4. Receptor Parameters

- Receptor: 1

- Room: 1

- Time fraction: 0.67

- Breathing Rate: $18 \mathrm{~m}^{3} / \mathrm{d}$

- Ingestion Rate: $0.0001 \mathrm{~m}^{2} / \mathrm{h}$

- Location:

- X: .915, Y: 2.5, Z: .6

5. Shielding Parameters

- Sources 2-6 / Receptor 1

o Thickness: $0.1 \mathrm{~cm}$

o Density: $7.6 \mathrm{~g} / \mathrm{cc}$

o Material: Iron

- $\quad$ Source 1 / Receptor 1

o Thickness: $0 \mathrm{~cm}$

o Density: $7.6 \mathrm{~g} / \mathrm{cc}$

o Material: Concrete 
6. Source Parameters

- Area sources

- Details (source 1 )

- Rectangular Geometry

o Length along $(\mathrm{m}) \mathrm{Y}: 1.2, \mathrm{Z}: 1.2$

o Release

- Air fraction: 0.1

- Direct Ingestion: 0 1/h

- Removable fraction: 0.1

- Lifetime (d): 365

o Radionuclide: Sr-90 Activity: $1.5 \mathrm{E} 5 \mathrm{dpm} / \mathrm{m}^{2}$

o Radionuclide: Cs-137 Activity: $1.5 E 5 \mathrm{dpm} / \mathrm{m}^{2}$

o Radionuclide: U-235 Activity: $8.0 \mathrm{E} 3 \mathrm{dpm} / \mathrm{m}^{2}$

- Details (source 2)

o Rectangular Geometry

o Length along $(\mathrm{m}) \mathrm{Y}: 1.2, \mathrm{Z}: 1.2$

o Release

- Air fraction: 0.1

- Direct Ingestion: 0 1/h

- Removable fraction: 0.1

- Lifetime (d): 365

o Radionuclide: Sr-90 Activity: $1.1 \mathrm{E} 6 \mathrm{dpm} / \mathrm{m}^{2}$

o Radionuclide: Cs-137 Activity: $1.1 \mathrm{E} 6 \mathrm{dpm} / \mathrm{m}^{2}$

o Radionuclide: U-235 Activity: $8.0 \mathrm{E} 3 \mathrm{dpm} / \mathrm{m}^{2}$

- Details (source 3-6)

- Rectangular Geometry

o Length along $(\mathrm{m}) \mathrm{X}: 1.2, \mathrm{Y}$ or Z: 1.83

o Release

- Air fraction: 0.1

- Direct Ingestion: 0 1/h

- Removable fraction: 0.1

- Lifetime (d): 365

o Radionuclide: Sr-90 Activity: $1.1 \mathrm{E} 6 \mathrm{dpm} / \mathrm{m}^{2}$

o Radionuclide: Cs-137 Activity: $1.1 \mathrm{E} 6 \mathrm{dpm} / \mathrm{m}^{2}$

o Radionuclide: U-235 Activity: $8.0 \mathrm{E} 3 \mathrm{dpm} / \mathrm{m}^{2}$

- Source locations

\begin{tabular}{|c|c|c|c|}
\hline Source Number & $X$ & $Y$ & $Z$ \\
\hline 1 & 1.82 & 1.6 & .6 \\
\hline 2 & 0 & 1.6 & .6 \\
\hline 3 & .915 & 1 & .6 \\
\hline 4 & .915 & 2.2 & .6 \\
\hline 5 & .915 & 1.6 & 0 \\
\hline 6 & .915 & 1.6 & 1.2 \\
\hline
\end{tabular}




\section{List of Assumptions and Parameters for Worst Case Scenario 2}

Any parameter that is not specifically mentioned uses the RESRAD-BUILD default value.

1. Case

- Case

o Dose Risk Library: ICRP 72 (Adult)

- Time Parameters

o Exposure Duration (days): 1

o Indoor fraction: 0.5

- Evaluation Times - Time Integration

o Maximum number of points for Dose/Risk: 17

2. Building Parameters

- Number of Rooms: 1

- Deposition Velocity: $0.01 \mathrm{~m} / \mathrm{s}$

- Resuspension Rate: 0.0000005 (1/s)

- Air Flow

o Building Exchange Rate: $0.8(1 / \mathrm{h})$

o Room 1

- Area $\left(\mathrm{m}^{2}\right): 36$

- Height (m): 2.5

3. Radiological Units

- Activity: dpm

- Dose: mrem

4. Receptor Parameters

- Receptor: 1

- Room: 1

- Time fraction: 0.5

- Breathing Rate: $18 \mathrm{~m}^{3} / \mathrm{d}$

- Ingestion Rate: $0.0001 \mathrm{~m}^{2} / \mathrm{h}$

- Location:

- $\mathrm{X}: 1, \mathrm{Y}: 1, \mathrm{Z}: 1$

5. Shielding Parameters

- Source/Receptor

- Thickness: $0 \mathrm{~cm}$

- Density: $2.4 \mathrm{~g} / \mathrm{cc}$

- Material: Concrete

6. Source Parameters

- Area sources

- Details

o Rectangular Geometry

o Length along $(\mathrm{m}) \mathrm{Y}: 1, \mathrm{Z}: 1$

o Release

- Air fraction: 0.5

- Direct Ingestion: $0.11 / \mathrm{h}$

- Removable fraction: 1

- Lifetime (d): 0.25

o Radionuclide: Sr-90 Activity: $1.5 \mathrm{E} 5 \mathrm{dpm} / \mathrm{m}^{2}$

o Radionuclide: Cs-137 Activity: $1.5 \mathrm{E} 5 \mathrm{dpm} / \mathrm{m}^{2}$

o Radionuclide: U-235 Activity: $8.0 \mathrm{E} 3 \mathrm{dpm} / \mathrm{m}^{2}$ 
* RESRAD-BUILD Dose Program Output, Version 3.50 05/04/10 10:22:18 Page: 1 ** Title : Likely Use 1 - Enthusiast

Input File : sitel.bld

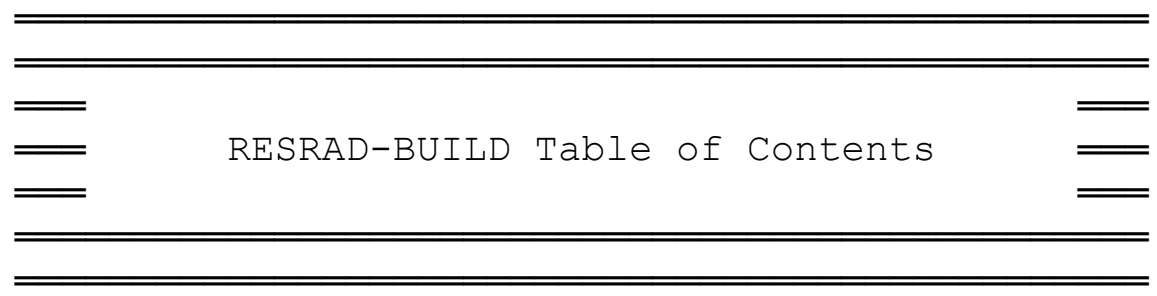

RESRAD-BUILD Input Parameters......... 2

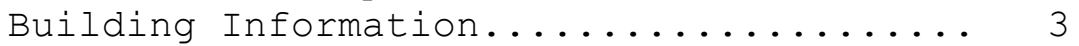

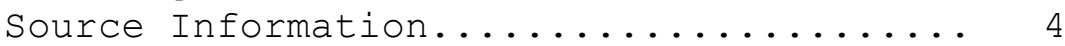

For time $=0.00 \mathrm{E}+00 \mathrm{yr}$

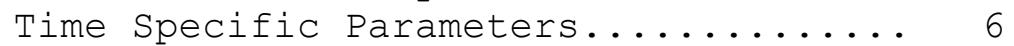

Receptor-Source Dose Summary........ 7

Dose by Pathway Detail............ 8

Dose by Nuclide Detail............ 9

For time $=1.00 \mathrm{E}+00 \mathrm{yr}$

Time Specific Parameters........... 10

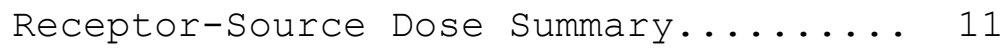

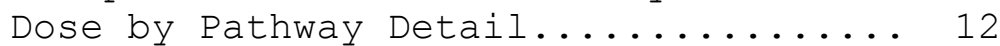

Dose by Nuclide Detail........... 13

For time $=1.00 \mathrm{E}+01 \mathrm{yr}$

Time Specific Parameters........... 14

Receptor-Source Dose Summary.......... 15

Dose by Pathway Detail............. 16

Dose by Nuclide Detail............ 17

For time $=3.00 \mathrm{E}+01 \mathrm{yr}$

Time Specific Parameters........... 18

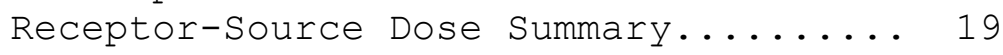

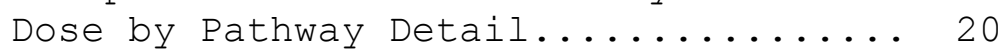

Dose by Nuclide Detail............ 21

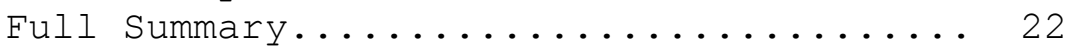


* RESRAD-BUILD Dose Program Output, Version 3.50 05/04/10 10:22:18 Page: 2 ** Title : Likely Use 1 - Enthusiast

Input File : sitel.bld

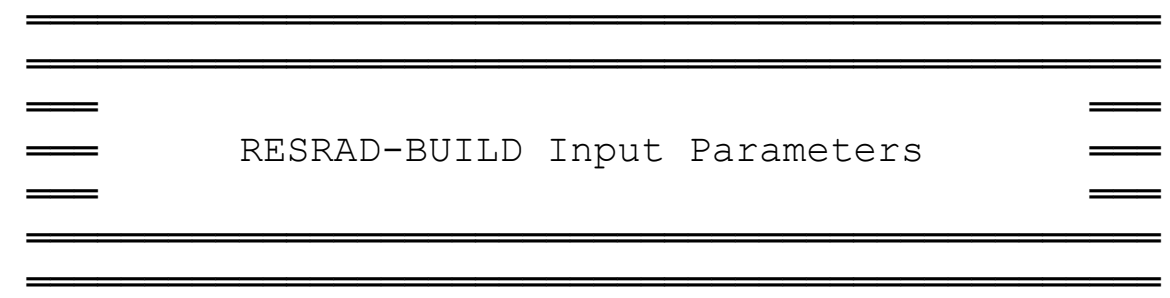

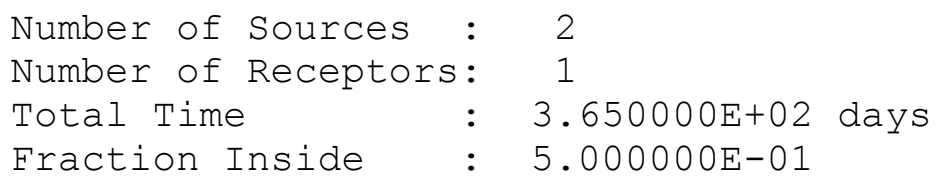

$\overline{\underline{ }}$ Receptor Information $\overline{=}$

Receptor

$\begin{array}{ccccccc}\text { Room } & \mathrm{x} & \mathrm{y} & \mathrm{z} & \text { Fractime } & \text { Inhalation } & \text { Ingestion(Dust) } \\ & {[\mathrm{m}]} & {[\mathrm{m}]} & {[\mathrm{m}]} & & {[\mathrm{m} / \mathrm{day}]} & {[\mathrm{m} 2 / \mathrm{hr}]} \\ 1 & 1.300 & 1.000 & 1.000 & 0.025 & 1.80 \mathrm{E}+01 & 1.00 \mathrm{E}-04\end{array}$

$=$ Receptor-Source Shielding Relationship $\overline{\overline{ }}$

Receptor Source Density Thickness Material

$[\mathrm{g} / \mathrm{cm} 3] \quad[\mathrm{cm}]$

\begin{tabular}{lllll}
\hline 1 & 1 & $7.60 \mathrm{E}+00$ & $4.00 \mathrm{E}-01$ & $\mathrm{Fe}$ \\
1 & 2 & $7.60 \mathrm{E}+00$ & $0.00 \mathrm{E}+00$ & $\mathrm{Fe}$
\end{tabular}


* RESRAD-BUILD Dose Program Output, Version 3.50 05/04/10 10:22:18 Page: 3 ** Title : Likely Use 1 - Enthusiast

Input File : sitel.bld

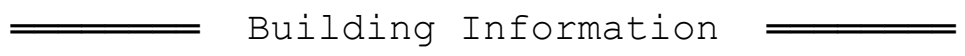

Building Air Exchange Rate: 8.00E-01 1/hr

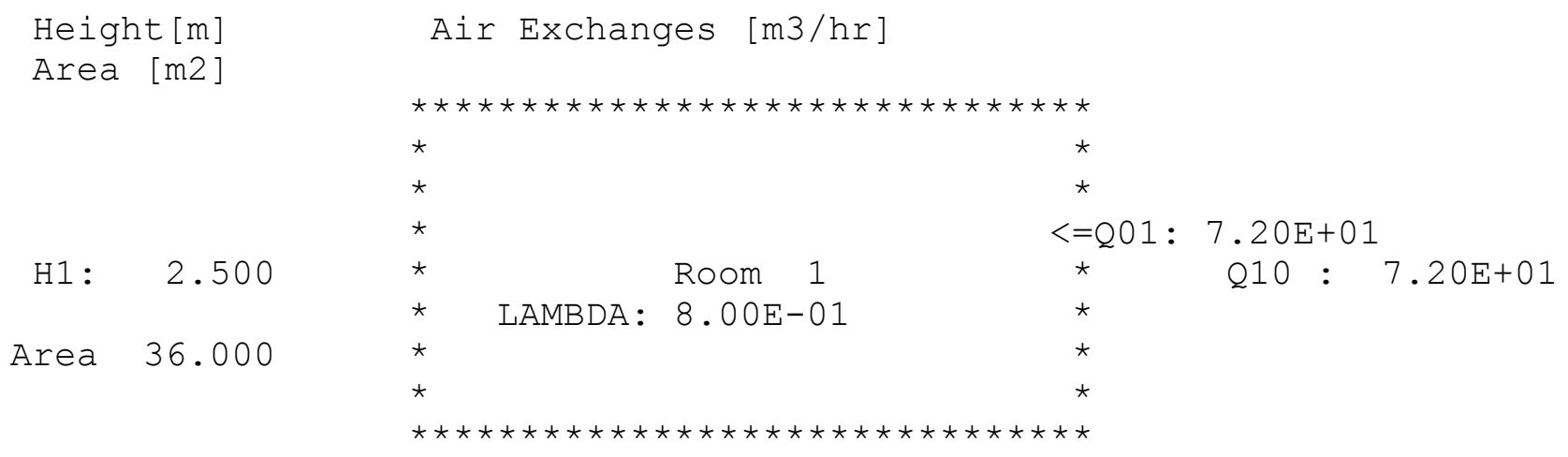

Deposition velocity: 1.00E-02 [m/s] Resuspension Rate: 5.00E-07 [1/s] 
RESRAD-BUILD Dose Program Output, Version 3.50 05/04/10 10:22:18 Page: 4 ** Title : Likely Use 1 - Enthusiast

Input File : sitel.bld

$=$ Source Information

Source: 1

Location: : Room : $1 \mathrm{x}: 1.00 \mathrm{y}: 1.00 \mathrm{z}: 1.00[\mathrm{~m}]$

Geometry:: Type: Area Length $[\mathrm{m}]: 3.66 \mathrm{E}+00$ Width $[\mathrm{m}]: 2.44 \mathrm{E}+00$ Direction: $x$

Pathway : :

Direct Ingestion Rate: $0.000 \mathrm{E}+00$ [1/hr]

Fraction released to air: 1.000E-01

Removable fraction: $\quad 1.000 \mathrm{E}-02$

Time to Remove: $\quad 3.650 \mathrm{E}+02$ [day]

Contamination: :

Nuclide Concentration Dose Conversion Factor (Library: ICRP 72 (Adult))

\begin{tabular}{|c|c|c|c|c|}
\hline & {$[\mathrm{dpm} / \mathrm{m} 2]$} & $\begin{array}{l}\text { Ingestion } \\
\text { [mrem/dpm }]\end{array}$ & $\begin{array}{l}\text { Inhalation } \\
\text { [mrem/dpm }\end{array}$ & $\begin{array}{l}\text { Submersion } \\
{[\mathrm{mrem} / \mathrm{yr} /} \\
(\mathrm{dpm} / \mathrm{m} 3)]\end{array}$ \\
\hline$U-235$ & $8.000 \mathrm{E}+03$ & $7.890 \mathrm{E}-05$ & $1.417 E-02$ & $4.063 E-04$ \\
\hline$P A-231$ & $0.000 E+00$ & $1.183 E-03$ & $2.333 E-01$ & $9.049 E-05$ \\
\hline$A C-227$ & $0.000 \mathrm{E}+00$ & $2.015 E-03$ & $9.476 E-01$ & $9.734 E-04$ \\
\hline$C S-137$ & $1.100 \mathrm{E}+06$ & $2.167 E-05$ & $6.500 E-05$ & $1.434 E-03$ \\
\hline$S R-90$ & $1.100 \mathrm{E}+06$ & $5.117 E-05$ & $2.692 E-04$ & $1.039 \mathrm{E}-05$ \\
\hline
\end{tabular}

Source: 2

Location: : Room : $1 \mathrm{x}: 1.60 \mathrm{y}: 1.00 \mathrm{z}: 1.00[\mathrm{~m}]$

Geometry:: Type: Area Length $[\mathrm{m}]: 1.00 \mathrm{E}+00$ Width $[\mathrm{m}]: 1.00 \mathrm{E}+00$ Direction: $\mathrm{x}$

Pathway : :

Direct Ingestion Rate: $0.000 \mathrm{E}+00$ [1/hr]

Fraction released to air: 1.000E-01

Removable fraction: 1.000E-01

Time to Remove: $\quad 3.650 \mathrm{E}+02$ [day]

Contamination: :

Nuclide Concentration Dose Conversion Factor (Library: ICRP 72 (Adult))

\begin{tabular}{|c|c|c|c|c|}
\hline & [dpm/m2 ] & $\begin{array}{l}\text { Ingestion } \\
\text { [mrem/dpm] }\end{array}$ & $\begin{array}{l}\text { Inhalation } \\
\text { [mrem/dpm ] }\end{array}$ & $\begin{array}{l}\text { Submersion } \\
{[\mathrm{mrem} / \mathrm{yr} /} \\
(\mathrm{dpm} / \mathrm{m} 3)]\end{array}$ \\
\hline$U-235$ & $8.000 E+03$ & $7.890 \mathrm{E}-05$ & $1.417 \mathrm{E}-02$ & $4.063 E-04$ \\
\hline
\end{tabular}


* RESRAD-BUILD Dose Program Output, Version 3.50 05/04/10 10:22:18 Page: 5 ** Title : Likely Use 1 - Enthusiast

Input File : sitel.bld

$\begin{array}{lllll}\mathrm{PA}-231 & 0.000 \mathrm{E}+00 & 1.183 \mathrm{E}-03 & 2.333 \mathrm{E}-01 & 9.049 \mathrm{E}-05 \\ \mathrm{AC}-227 & 0.000 \mathrm{E}+00 & 2.015 \mathrm{E}-03 & 9.476 \mathrm{E}-01 & 9.734 \mathrm{E}-04 \\ \mathrm{CS}-137 & 1.500 \mathrm{E}+05 & 2.167 \mathrm{E}-05 & 6.500 \mathrm{E}-05 & 1.434 \mathrm{E}-03 \\ \mathrm{SR}-90 & 1.500 \mathrm{E}+05 & 5.117 \mathrm{E}-05 & 2.692 \mathrm{E}-04 & 1.039 \mathrm{E}-05\end{array}$


* RESRAD-BUILD Dose Program Output, Version 3.50 05/04/10 10:22:18 Page: 6 ** Title : Likely Use 1 - Enthusiast

Input File : sitel.bld

Evaluation Time: $0.00000000 \mathrm{E}+00$ years

\begin{tabular}{rrrrr}
\hline \hline & & & & \\
\hline \hline & Assessment for Time: & 1 & $=$ \\
\hline \hline & Time & $=0.00 \mathrm{E}+00$ & yr & \\
\hline \hline
\end{tabular}

$=$ Source Information

Source: 1

Location: : Room : $1 \mathrm{x}: 1.00 \mathrm{y}: 1.00 \mathrm{z}: 1.00$ [m]

Geometry:: Type: Area Length $[\mathrm{m}]: 3.66 \mathrm{E}+00$ Width $[\mathrm{m}]: 2.44 \mathrm{E}+00$ Direction: $\mathrm{x}$

Pathway : :

Direct Ingestion Rate: $0.000 \mathrm{E}+00$ [1/hr]

Fraction released to air: 1.000E-01

Removable fraction: $\quad 1.000 \mathrm{E}-02$

Time to Remove: 3.650E+02 [day]

\begin{tabular}{|c|c|c|}
\hline \multirow[t]{6}{*}{ Contamination: : } & Nuclide & $\begin{array}{c}\text { Concentration } \\
{[\mathrm{dpm} / \mathrm{m} 2]}\end{array}$ \\
\hline & $U-235$ & $8.000 \mathrm{E}+03$ \\
\hline & $\mathrm{PA}-231$ & $0.000 \mathrm{E}+00$ \\
\hline & $A C-227$ & $0.000 \mathrm{E}+00$ \\
\hline & $C S-137$ & $1.100 \mathrm{E}+06$ \\
\hline & $S R-90$ & $1.100 \mathrm{E}+06$ \\
\hline
\end{tabular}

Source: 2

Location: : Room : $1 \mathrm{x}: 1.60 \mathrm{y}: 1.00 \mathrm{z}: 1.00[\mathrm{~m}]$

Geometry:: Type: Area Length $[\mathrm{m}]: 1.00 \mathrm{E}+00$ Width $[\mathrm{m}]: 1.00 \mathrm{E}+00$ Direction: $\mathrm{x}$

Pathway : :

Direct Ingestion Rate: $0.000 \mathrm{E}+00$ [1/hr]

Fraction released to air: 1.000E-01

Removable fraction: $\quad 1.000 \mathrm{E}-01$

Time to Remove: 3.650E+02 [day]

Contamination: : Nuclide Concentration

$\begin{array}{ll}\mathrm{U}-235 & 8.000 \mathrm{E}+03 \\ \mathrm{PA}-231 & 0.000 \mathrm{E}+00 \\ \mathrm{AC}-227 & 0.000 \mathrm{E}+00 \\ \mathrm{CS}-137 & 1.500 \mathrm{E}+05 \\ \mathrm{SR}-90 & 1.500 \mathrm{E}+05\end{array}$


* RESRAD-BUILD Dose Program Output, Version 3.50 05/04/10 10:22:18 Page: 7 ** Title : Likely Use 1 - Enthusiast

Input File : sitel.bld

Evaluation Time: $0.00000000 \mathrm{E}+00$ years

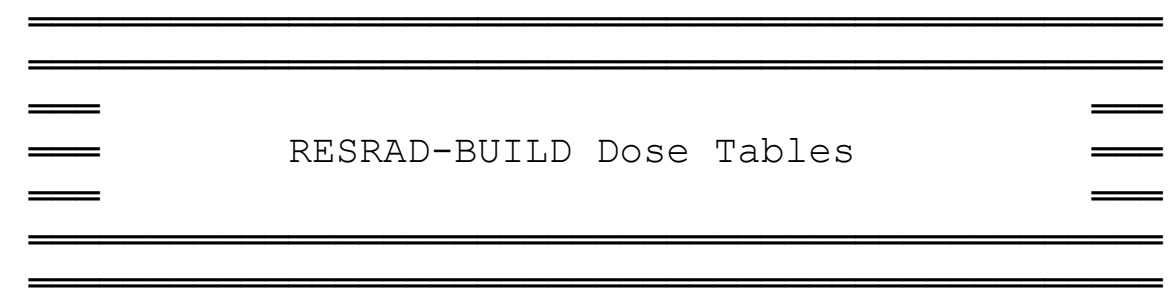

Source Contributions to Receptor Doses

=

[mrem]

Source Source Total

12

Receptor 1 1.01E-01 7.78E-03 1.09E-01

Total 1.01E-01 7.78E-03 1.09E-01 
* RESRAD-BUILD Dose Program Output, Version 3.50 05/04/10 10:22:18 Page: 8 ** Title : Likely Use 1 - Enthusiast

Input File : sitel.bld

Evaluation Time: $0.00000000 \mathrm{E}+00$ years

Pathway Detail of Doses

[mrem]

Source: 1

Receptor

1

Total

External

Deposition Immersion

Inhalation

Radon

2. 66 E-07

$5.32 \mathrm{E}-04$

$0.00 E+00$

Ingestion

1. $00 \mathrm{E}-01$

$5.42 E-05$

$2.66 \mathrm{E}-07$

$5.32 \mathrm{E}-04$

$0.00 \mathrm{E}+00$

2. 36E-04

$2.36 \mathrm{E}-04$

Source: 2

Receptor

1

Total
External

7. 53E-03

7. $53 \mathrm{E}-03$
Deposition
$8.40 \mathrm{E}-06$

8. $40 \mathrm{E}-06$
Immersion

4.12E-08

4. $12 \mathrm{E}-08$
Inhalation

2.06E-04

2. $06 \mathrm{E}-04$
Radon

$0.00 E+00$

$0.00 \mathrm{E}+00$
Ingestion

3.78E-05

3. $78 \mathrm{E}-05$ 
** RESRAD-BUILD Dose Program Output, Version 3.50 05/04/10 10:22:18 Page: 9 ** Title : Likely Use 1 - Enthusiast

Input File : sitel.bld

Evaluation Time: $0.00000000 \mathrm{E}+00$ years

Nuclide Detail of Doses

[mrem ]

Source: 1

$\begin{array}{lcc}\text { Nuclide } & \begin{array}{c}\text { Receptor } \\ 1\end{array} & \text { Total } \\ \text { U-235 } & 2.47 \mathrm{E}-04 & 2.47 \mathrm{E}-04 \\ \mathrm{PA}-231 & 2.27 \mathrm{E}-08 & 2.27 \mathrm{E}-08 \\ \mathrm{AC}-227 & 9.33 \mathrm{E}-10 & 9.33 \mathrm{E}-10 \\ \mathrm{CS}-137 & 9.97 \mathrm{E}-02 & 9.97 \mathrm{E}-02 \\ \mathrm{SR}-90 & 1.29 \mathrm{E}-03 & 1.29 \mathrm{E}-03\end{array}$

Source: 2

Nuclide Receptor Total

1

U-235 2.73E-04 2.73E-04

$\begin{array}{lll}\mathrm{PA}-231 & 2.53 \mathrm{E}-08 & 2.53 \mathrm{E}-08\end{array}$

$\begin{array}{lll}\mathrm{AC}-227 & 1.03 \mathrm{E}-09 & 1.03 \mathrm{E}-09\end{array}$

$\begin{array}{lll}C S-137 & 7.36 E-03 & 7.36 E-03\end{array}$

$\begin{array}{lll}\mathrm{SR}-90 & 1.49 \mathrm{E}-04 & 1.49 \mathrm{E}-04\end{array}$ 
* RESRAD-BUILD Dose Program Output, Version 3.50 05/04/10 10:22:18 Page: 10 ** Title : Likely Use 1 - Enthusiast

Input File : sitel.bld

Evaluation Time: 1.00000000 years

\begin{tabular}{rrrrr}
\hline \hline & & & & \\
\hline \hline & Assessment for Time: & 2 & $=$ \\
Time & $=1.00 \mathrm{E}+00$ & $\mathrm{yr}$ & $=$ \\
\hline \hline
\end{tabular}

$=$ Source Information

Source: 1

Location: : Room : $1 \mathrm{x}: 1.00 \mathrm{y}: 1.00 \mathrm{z}: 1.00$ [m]

Geometry:: Type: Area Length $[\mathrm{m}]: 3.66 \mathrm{E}+00$ Width $[\mathrm{m}]: 2.44 \mathrm{E}+00$ Direction: $\mathrm{x}$

Pathway : :

Direct Ingestion Rate: $0.000 \mathrm{E}+00$ [1/hr]

Fraction released to air: 1.000E-01

Removable fraction: $\quad 0.000 \mathrm{E}+00$

Time to Remove: 3.650E+02 [day]

\begin{tabular}{|c|c|c|}
\hline \multirow[t]{6}{*}{ Contamination: : } & Nuclide & $\begin{array}{c}\text { Concentration } \\
{[\mathrm{dpm} / \mathrm{m} 2]}\end{array}$ \\
\hline & $U-235$ & $7.920 \mathrm{E}+03$ \\
\hline & $\mathrm{PA}-231$ & $1.674 \mathrm{E}-01$ \\
\hline & $A C-227$ & $2.633 E-03$ \\
\hline & $C S-137$ & $1.064 \mathrm{E}+06$ \\
\hline & $S R-90$ & $1.063 E+06$ \\
\hline
\end{tabular}

Source: 2

Location: : Room : $1 \mathrm{x}: 1.60 \mathrm{y}: 1.00 \mathrm{z}: 1.00[\mathrm{~m}]$

Geometry:: Type: Area Length $[\mathrm{m}]: 1.00 \mathrm{E}+00$ Width $[\mathrm{m}]: 1.00 \mathrm{E}+00$ Direction: $\mathrm{x}$

Pathway : :

Direct Ingestion Rate: $0.000 \mathrm{E}+00$ [1/hr]

Fraction released to air: 1.000E-01

Removable fraction: $\quad 0.000 \mathrm{E}+00$

Time to Remove: $\quad 3.650 \mathrm{E}+02$ [day]

Contamination: : Nuclide Concentration

$\begin{array}{ll}\mathrm{U}-235 & 7.200 \mathrm{E}+03 \\ \mathrm{PA}-231 & 1.522 \mathrm{E}-01 \\ \mathrm{AC}-227 & 2.393 \mathrm{E}-03 \\ \mathrm{CS}-137 & 1.319 \mathrm{E}+05 \\ \mathrm{SR}-90 & 1.318 \mathrm{E}+05\end{array}$ 
** RESRAD-BUILD Dose Program Output, Version 3.50 05/04/10 10:22:18 Page: 11 ** Title : Likely Use 1 - Enthusiast

Input File : sitel.bld

Evaluation Time: 1.00000000 years

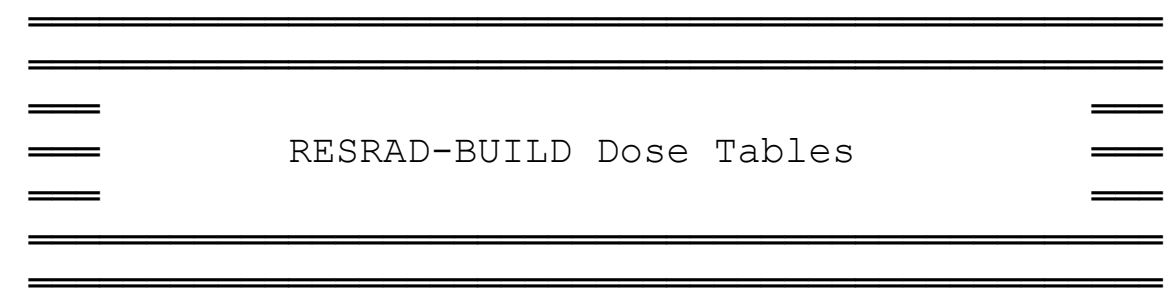

Source Contributions to Receptor Doses

=

[mrem]

Source Source Total

12

Receptor $19.76 \mathrm{E}-02 \quad 6.97 \mathrm{E}-03 \quad 1.05 \mathrm{E}-01$

Total 9.76E-02 6.97E-03 $1.05 \mathrm{E}-01$ 
* RESRAD-BUILD Dose Program Output, Version 3.50 05/04/10 10:22:18 Page: 12 ** Title : Likely Use 1 - Enthusiast

Input File : sitel.bld

Evaluation Time: 1.00000000 years

Pathway Detail of Doses

[mrem $]$

Source: 1

$\begin{array}{cllllll}\text { Receptor } & \text { External } & \text { Deposition } & \text { Immersion } & \text { Inhalation } & \text { Radon } & \text { Ingestion } \\ 1 & 9.76 \mathrm{E}-02 & 0.00 \mathrm{E}+00 & 0.00 \mathrm{E}+00 & 0.00 \mathrm{E}+00 & 0.00 \mathrm{E}+00 & 0.00 \mathrm{E}+00 \\ \text { Total } & 9.76 \mathrm{E}-02 & 0.00 \mathrm{E}+00 & 0.00 \mathrm{E}+00 & 0.00 \mathrm{E}+00 & 0.00 \mathrm{E}+00 & 0.00 \mathrm{E}+00\end{array}$

Source: 2

$\begin{array}{cllllll}\text { Receptor } & \text { External } & \text { Deposition } & \text { Immersion } & \text { Inhalation } & \text { Radon } & \text { Ingestion } \\ 1 & 6.97 \mathrm{E}-03 & 0.00 \mathrm{E}+00 & 0.00 \mathrm{E}+00 & 0.00 \mathrm{E}+00 & 0.00 \mathrm{E}+00 & 0.00 \mathrm{E}+00 \\ \text { Total } & 6.97 \mathrm{E}-03 & 0.00 \mathrm{E}+00 & 0.00 \mathrm{E}+00 & 0.00 \mathrm{E}+00 & 0.00 \mathrm{E}+00 & 0.00 \mathrm{E}+00\end{array}$


* RESRAD-BUILD Dose Program Output, Version 3.50 05/04/10 10:22:18 Page: 13 ** Title : Likely Use 1 - Enthusiast

Input File : sitel.bld

Evaluation Time: 1.00000000 years

Nuclide Detail of Doses

[mrem]

Source: 1

$\begin{array}{lcc}\text { Nuclide } & \begin{array}{c}\text { Receptor } \\ 1\end{array} & \text { Total } \\ \text { U-235 } & 1.16 \mathrm{E}-04 & 1.16 \mathrm{E}-04 \\ \mathrm{PA}-231 & 1.27 \mathrm{E}-09 & 1.27 \mathrm{E}-09 \\ \mathrm{AC}-227 & 2.94 \mathrm{E}-10 & 2.94 \mathrm{E}-10 \\ \mathrm{CS}-137 & 9.67 \mathrm{E}-02 & 9.67 \mathrm{E}-02 \\ \mathrm{SR}-90 & 7.82 \mathrm{E}-04 & 7.82 \mathrm{E}-04\end{array}$

Source: 2

Nuclide Receptor Total

1

U-235 $1.19 \mathrm{E}-04 \quad 1.19 \mathrm{E}-04$

$\begin{array}{lll}\mathrm{PA}-231 & 1.09 \mathrm{E}-09 & 1.09 \mathrm{E}-09\end{array}$

$\mathrm{AC}-227 \quad 2.03 \mathrm{E}-10 \quad 2.03 \mathrm{E}-10$

$\begin{array}{lll}\mathrm{C} S-137 & 6.78 \mathrm{E}-03 & 6.78 \mathrm{E}-03\end{array}$

$\begin{array}{lll}\mathrm{SR}-90 & 6.87 \mathrm{E}-05 & 6.87 \mathrm{E}-05\end{array}$ 
* RESRAD-BUILD Dose Program Output, Version 3.50 05/04/10 10:22:18 Page: 14 ** Title : Likely Use 1 - Enthusiast

Input File : sitel.bld

Evaluation Time: 10.0000000 years

\begin{tabular}{rrrrr}
\hline \hline & & & & \\
\hline \hline & Assessment for Time: & 3 & $=$ \\
Time & $=1.00 \mathrm{E}+01$ & yr & $=$ \\
\hline \hline
\end{tabular}

$=$ Source Information

Source: 1

Location: : Room : $1 \mathrm{x}: 1.00 \mathrm{y}: 1.00 \mathrm{z}: 1.00$ [m]

Geometry:: Type: Area Length $[\mathrm{m}]: 3.66 \mathrm{E}+00$ Width $[\mathrm{m}]: 2.44 \mathrm{E}+00$ Direction: $\mathrm{x}$

Pathway : :

Direct Ingestion Rate: $0.000 \mathrm{E}+00$ [1/hr]

Fraction released to air: 1.000E-01

Removable fraction: $\quad 0.000 \mathrm{E}+00$

Time to Remove: 3.650E+02 [day]

\begin{tabular}{|c|c|c|}
\hline \multirow[t]{6}{*}{ Contamination: : } & Nuclide & $\begin{array}{c}\text { Concentration } \\
{[\mathrm{dpm} / \mathrm{m} 2]}\end{array}$ \\
\hline & $U-235$ & $7.920 E+03$ \\
\hline & $\mathrm{PA}-231$ & $1.674 \mathrm{E}+00$ \\
\hline & $A C-227$ & $2.400 \mathrm{E}-01$ \\
\hline & $C S-137$ & $8.643 E+05$ \\
\hline & $S R-90$ & $8.582 E+05$ \\
\hline
\end{tabular}

Source: 2

Location: : Room : $1 \mathrm{x}: 1.60 \mathrm{y}: 1.00 \mathrm{z}: 1.00[\mathrm{~m}]$

Geometry:: Type: Area Length $[\mathrm{m}]: 1.00 \mathrm{E}+00$ Width $[\mathrm{m}]: 1.00 \mathrm{E}+00$ Direction: $\mathrm{x}$

Pathway : :

Direct Ingestion Rate: $0.000 \mathrm{E}+00$ [1/hr]

Fraction released to air: 1.000E-01

Removable fraction: $\quad 0.000 \mathrm{E}+00$

Time to Remove: $\quad 3.650 \mathrm{E}+02$ [day]

Contamination: : Nuclide Concentration

$\begin{array}{ll}\mathrm{U}-235 & 7.200 \mathrm{E}+03 \\ \mathrm{PA}-231 & 1.521 \mathrm{E}+00 \\ \mathrm{AC}-227 & 2.182 \mathrm{E}-01 \\ \mathrm{CS}-137 & 1.071 \mathrm{E}+05 \\ \mathrm{SR}-90 & 1.064 \mathrm{E}+05\end{array}$ 
* RESRAD-BUILD Dose Program Output, Version 3.50 05/04/10 10:22:18 Page: 15 ** Title : Likely Use 1 - Enthusiast

Input File : sitel.bld

Evaluation Time: 10.0000000 years

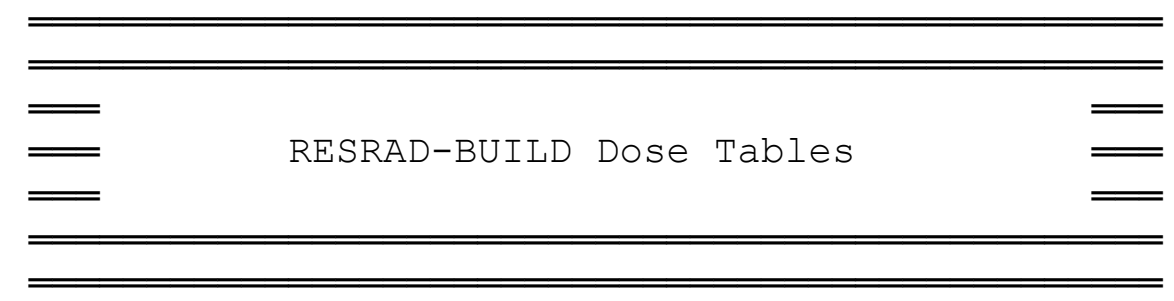

Source Contributions to Receptor Doses

=

[mrem]

Source Source Total

12

Receptor 1 7.93E-02 5.68E-03 8.50E-02

Total 7.93E-02 $5.68 \mathrm{E}-038.50 \mathrm{E}-02$ 
* RESRAD-BUILD Dose Program Output, Version 3.50 05/04/10 10:22:18 Page: 16 ** Title : Likely Use 1 - Enthusiast

Input File : sitel.bld

Evaluation Time: 10.0000000 years

Pathway Detail of Doses

[mrem $]$

Source: 1

$\begin{array}{cllllll}\text { Receptor } & \text { External } & \text { Deposition } & \text { Immersion } & \text { Inhalation } & \text { Radon } & \text { Ingestion } \\ 1 & 7.93 \mathrm{E}-02 & 0.00 \mathrm{E}+00 & 0.00 \mathrm{E}+00 & 0.00 \mathrm{E}+00 & 0.00 \mathrm{E}+00 & 0.00 \mathrm{E}+00 \\ \text { Total } & 7.93 \mathrm{E}-02 & 0.00 \mathrm{E}+00 & 0.00 \mathrm{E}+00 & 0.00 \mathrm{E}+00 & 0.00 \mathrm{E}+00 & 0.00 \mathrm{E}+00\end{array}$

Source: 2

$\begin{array}{cllllll}\text { Receptor } & \text { External } & \text { Deposition } & \text { Immersion } & \text { Inhalation } & \text { Radon } & \text { Ingestion } \\ 1 & 5.68 \mathrm{E}-03 & 0.00 \mathrm{E}+00 & 0.00 \mathrm{E}+00 & 0.00 \mathrm{E}+00 & 0.00 \mathrm{E}+00 & 0.00 \mathrm{E}+00 \\ \text { Total } & 5.68 \mathrm{E}-03 & 0.00 \mathrm{E}+00 & 0.00 \mathrm{E}+00 & 0.00 \mathrm{E}+00 & 0.00 \mathrm{E}+00 & 0.00 \mathrm{E}+00\end{array}$


* RESRAD-BUILD Dose Program Output, Version 3.50 05/04/10 10:22:18 Page: 17 ** Title : Likely Use 1 - Enthusiast

Input File : sitel.bld

Evaluation Time: 10.0000000 years

Nuclide Detail of Doses

[mrem]

Source: 1

$\begin{array}{lcc}\text { Nuclide } & \begin{array}{c}\text { Receptor } \\ 1\end{array} & \text { Total } \\ \text { U-235 } & 1.16 \mathrm{E}-04 & 1.16 \mathrm{E}-04 \\ \mathrm{PA}-231 & 8.88 \mathrm{E}-09 & 8.88 \mathrm{E}-09 \\ \mathrm{AC}-227 & 1.27 \mathrm{E}-08 & 1.27 \mathrm{E}-08 \\ \mathrm{CS}-137 & 7.86 \mathrm{E}-02 & 7.86 \mathrm{E}-02 \\ \mathrm{SR}-90 & 6.31 \mathrm{E}-04 & 6.31 \mathrm{E}-04\end{array}$

Source: 2

Nuclide Receptor Total

1

$\begin{array}{lll}\mathrm{U}-235 & 1.19 \mathrm{E}-04 & 1.19 \mathrm{E}-04\end{array}$

$\begin{array}{lll}\mathrm{PA}-231 & 7.63 \mathrm{E}-09 & 7.63 \mathrm{E}-09\end{array}$

$\begin{array}{lll}\mathrm{AC}-227 & 8.78 \mathrm{E}-09 \quad 8.78 \mathrm{E}-09\end{array}$

CS-137 5.51E-03 5.51E-03

SR-90 5.55E-05 5.55E-05 
* RESRAD-BUILD Dose Program Output, Version 3.50 05/04/10 10:22:18 Page: 18 ** Title : Likely Use 1 - Enthusiast

Input File : sitel.bld

Evaluation Time: 30.0000000 years

\begin{tabular}{rrrrr}
\hline \hline & & & & \\
\hline \hline & Assessment for Time: & 4 & $=$ \\
Time & $=3.00 \mathrm{E}+01$ & yr & $=$ \\
\hline \hline
\end{tabular}

$=$ Source Information

Source: 1

Location: : Room : $1 \mathrm{x}: 1.00 \mathrm{y}: 1.00 \mathrm{z}: 1.00$ [m]

Geometry:: Type: Area Length $[\mathrm{m}]: 3.66 \mathrm{E}+00$ Width $[\mathrm{m}]: 2.44 \mathrm{E}+00$ Direction: $\mathrm{x}$

Pathway : :

Direct Ingestion Rate: $0.000 \mathrm{E}+00$ [1/hr]

Fraction released to air: 1.000E-01

Removable fraction: $\quad 0.000 \mathrm{E}+00$

Time to Remove: 3.650E+02 [day]

\begin{tabular}{|c|c|c|}
\hline \multirow[t]{6}{*}{ Contamination: : } & Nuclide & $\begin{array}{c}\text { Concentration } \\
{[\mathrm{dpm} / \mathrm{m} 2]}\end{array}$ \\
\hline & $U-235$ & $7.920 E+03$ \\
\hline & $\mathrm{PA}-231$ & $5.019 \mathrm{E}+00$ \\
\hline & $A C-227$ & $1.785 \mathrm{E}+00$ \\
\hline & $C S-137$ & $5.445 E+05$ \\
\hline & $S R-90$ & $5.330 E+05$ \\
\hline
\end{tabular}

Source: 2

Location: : Room : $1 \mathrm{x}: 1.60 \mathrm{y}: 1.00 \mathrm{z}: 1.00[\mathrm{~m}]$

Geometry:: Type: Area Length $[\mathrm{m}]: 1.00 \mathrm{E}+00$ Width $[\mathrm{m}]: 1.00 \mathrm{E}+00$ Direction: $\mathrm{x}$

Pathway : :

Direct Ingestion Rate: $0.000 \mathrm{E}+00$ [1/hr]

Fraction released to air: 1.000E-01

Removable fraction: $\quad 0.000 \mathrm{E}+00$

Time to Remove: $\quad 3.650 \mathrm{E}+02$ [day]

Contamination: : Nuclide Concentration

$\begin{array}{ll}\mathrm{U}-235 & 7.200 \mathrm{E}+03 \\ \mathrm{PA}-231 & 4.563 \mathrm{E}+00 \\ \mathrm{AC}-227 & 1.622 \mathrm{E}+00 \\ \mathrm{CS}-137 & 6.750 \mathrm{E}+04 \\ \mathrm{SR}-90 & 6.607 \mathrm{E}+04\end{array}$ 
* RESRAD-BUILD Dose Program Output, Version 3.50 05/04/10 10:22:18 Page: 19 ** Title : Likely Use 1 - Enthusiast

Input File : sitel.bld

Evaluation Time: 30.0000000 years

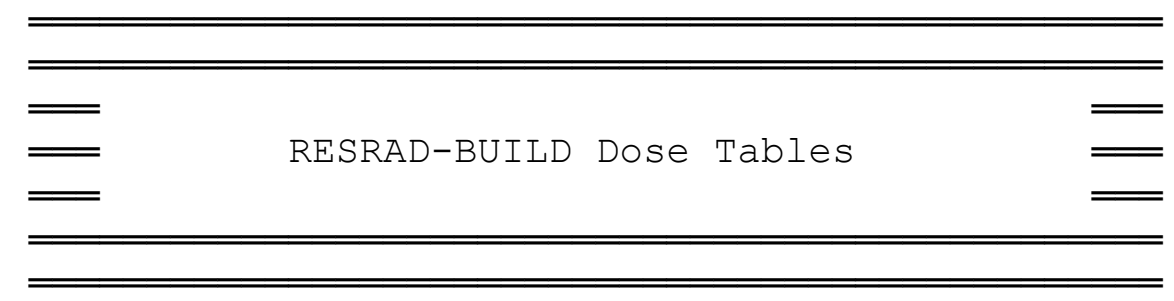

Source Contributions to Receptor Doses

=

[mrem]

Source Source Total

12

Receptor $15.00 \mathrm{E}-02 \quad 3.62 \mathrm{E}-03 \quad 5.36 \mathrm{E}-02$

Total 5.00E-02 3.62E-03 5.36E-02 
* RESRAD-BUILD Dose Program Output, Version 3.50 05/04/10 10:22:18 Page: 20 ** Title : Likely Use 1 - Enthusiast

Input File : sitel.bld

Evaluation Time: 30.0000000 years

Pathway Detail of Doses

[mrem $]$

Source: 1

$\begin{array}{cllllll}\text { Receptor } & \text { External } & \text { Deposition } & \text { Immersion } & \text { Inhalation } & \text { Radon } & \text { Ingestion } \\ 1 & 5.00 \mathrm{E}-02 & 0.00 \mathrm{E}+00 & 0.00 \mathrm{E}+00 & 0.00 \mathrm{E}+00 & 0.00 \mathrm{E}+00 & 0.00 \mathrm{E}+00 \\ \text { Total } & 5.00 \mathrm{E}-02 & 0.00 \mathrm{E}+00 & 0.00 \mathrm{E}+00 & 0.00 \mathrm{E}+00 & 0.00 \mathrm{E}+00 & 0.00 \mathrm{E}+00\end{array}$

Source: 2

$\begin{array}{cllllll}\text { Receptor } & \text { External } & \text { Deposition } & \text { Immersion } & \text { Inhalation } & \text { Radon } & \text { Ingestion } \\ 1 & 3.62 \mathrm{E}-03 & 0.00 \mathrm{E}+00 & 0.00 \mathrm{E}+00 & 0.00 \mathrm{E}+00 & 0.00 \mathrm{E}+00 & 0.00 \mathrm{E}+00 \\ \text { Total } & 3.62 \mathrm{E}-03 & 0.00 \mathrm{E}+00 & 0.00 \mathrm{E}+00 & 0.00 \mathrm{E}+00 & 0.00 \mathrm{E}+00 & 0.00 \mathrm{E}+00\end{array}$


* RESRAD-BUILD Dose Program Output, Version 3.50 05/04/10 10:22:18 Page: 21 ** Title : Likely Use 1 - Enthusiast

Input File : sitel.bld

Evaluation Time: 30.0000000 years

Nuclide Detail of Doses

[mrem]

Source: 1

$\begin{array}{lcc}\text { Nuclide } & \begin{array}{c}\text { Receptor } \\ 1\end{array} & \text { Total } \\ \text { U-235 } & 1.16 \mathrm{E}-04 & 1.16 \mathrm{E}-04 \\ \mathrm{PA}-231 & 2.58 \mathrm{E}-08 & 2.58 \mathrm{E}-08 \\ \mathrm{AC}-227 & 8.85 \mathrm{E}-08 & 8.85 \mathrm{E}-08 \\ \mathrm{CS}-137 & 4.95 \mathrm{E}-02 & 4.95 \mathrm{E}-02 \\ \mathrm{SR}-90 & 3.92 \mathrm{E}-04 & 3.92 \mathrm{E}-04\end{array}$

Source: 2

Nuclide Receptor Total

1

U-235 $1.19 \mathrm{E}-04 \quad 1.19 \mathrm{E}-04$

$\begin{array}{lll}\mathrm{PA}-231 & 2.22 \mathrm{E}-08 & 2.22 \mathrm{E}-08\end{array}$

$\begin{array}{lll}\mathrm{AC}-227 & 6.12 \mathrm{E}-08 & 6.12 \mathrm{E}-08\end{array}$

$\begin{array}{lll}C S-137 & 3.47 \mathrm{E}-03 & 3.47 \mathrm{E}-03\end{array}$

SR-90 3.45E-05 3.45E-05 
* RESRAD-BUILD Dose Program Output, Version 3.50 05/04/10 10:22:18 Page: 22 ** Title : Likely Use 1 - Enthusiast

Input File : sitel.bld

Full Summary

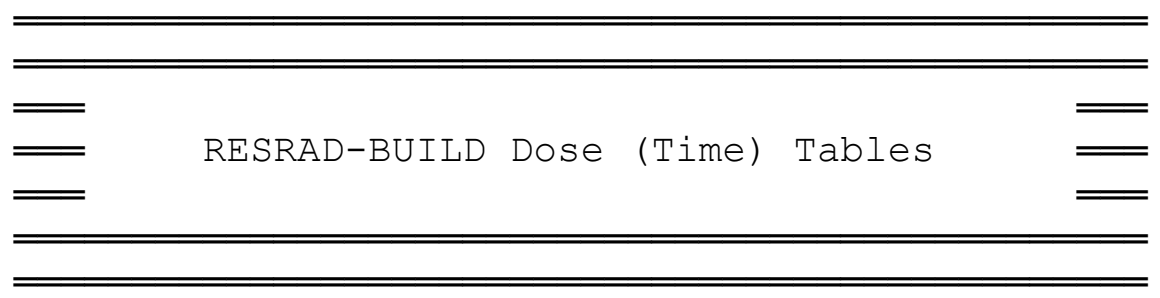

Receptor Dose Received for the Exposure Duration

=

(mrem)

Evaluation Time [yr]

$0.00 \mathrm{E}+00 \quad 1.00 \mathrm{E}+00 \quad 1.00 \mathrm{E}+01 \quad 3.00 \mathrm{E}+01$

$1 \quad 1.09 \mathrm{E}-01 \quad 1.05 \mathrm{E}-01 \quad 8.50 \mathrm{E}-02 \quad 5.36 \mathrm{E}-02$

Receptor Dose/Yr Averaged Over Exposure Duration

$(\mathrm{mrem} / \mathrm{yr})$

Evaluation Time [yr]

$0.00 \mathrm{E}+00 \quad 1.00 \mathrm{E}+00 \quad 1.00 \mathrm{E}+01 \quad 3.00 \mathrm{E}+01$

1 1.09E-01 1.05E-01 8.50E-02 5.37E-02 
* RESRAD-BUILD Dose Program Output, Version 3.50 05/04/10 10:52:40 Page: 1 ** Title : Likely Use 2 - Employee

Input File : sitel.bld

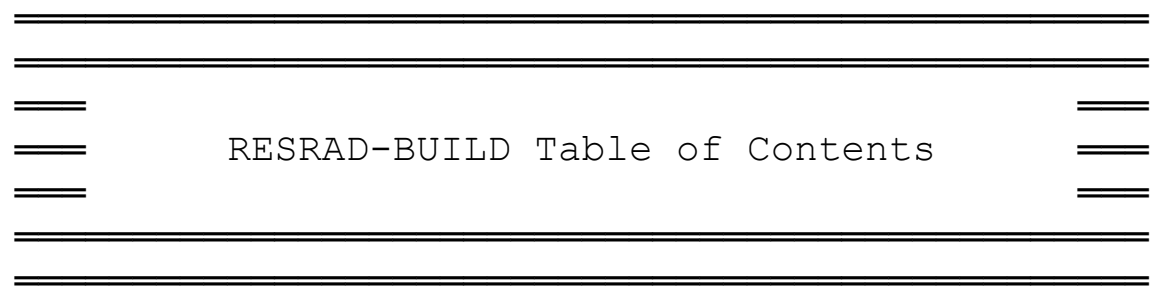

RESRAD-BUILD Input Parameters......... 2

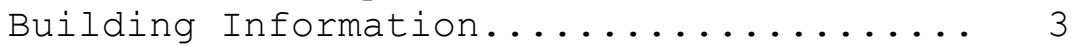

Source Information............... 4

For time $=0.00 \mathrm{E}+00 \mathrm{yr}$

Time Specific Parameters.............. 6

Receptor-Source Dose Summary........ 8

Dose by Pathway Detail............ 9

Dose by Nuclide Detail............ 10

For time $=1.00 \mathrm{E}+00 \mathrm{yr}$

Time Specific Parameters........... 11

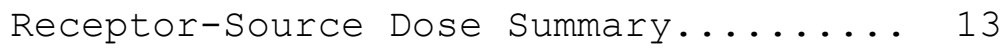

Dose by Pathway Detail............ 14

Dose by Nuclide Detail............ 15

For time $=1.00 \mathrm{E}+01 \mathrm{yr}$

Time Specific Parameters.......... 16

Receptor-Source Dose Summary......... 18

Dose by Pathway Detail............. 19

Dose by Nuclide Detail............ 20

For time $=3.00 \mathrm{E}+01 \mathrm{yr}$

Time Specific Parameters........... 21

Receptor-Source Dose Summary......... 23

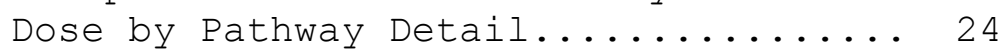

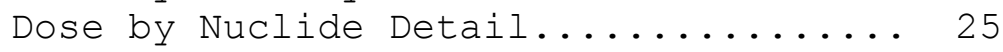

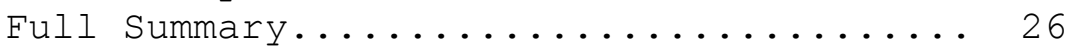


* RESRAD-BUILD Dose Program Output, Version 3.50 05/04/10 10:52:40 Page: 2 ** Title : Likely Use 2 - Employee

Input File : sitel.bld

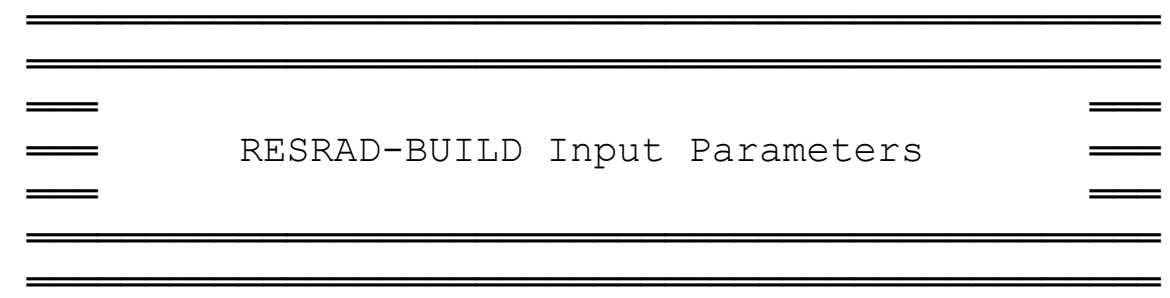

$\begin{array}{lcc}\text { Number of Sources }: & 3 \\ \text { Number of Receptors: } & 1 \\ \text { Total Time } & 3.650000 \mathrm{E}+02 \text { days } \\ \text { Fraction Inside }: & 5.000000 \mathrm{E}-01\end{array}$

$\overline{\underline{ }}$ Receptor Information $\overline{=}$

Receptor

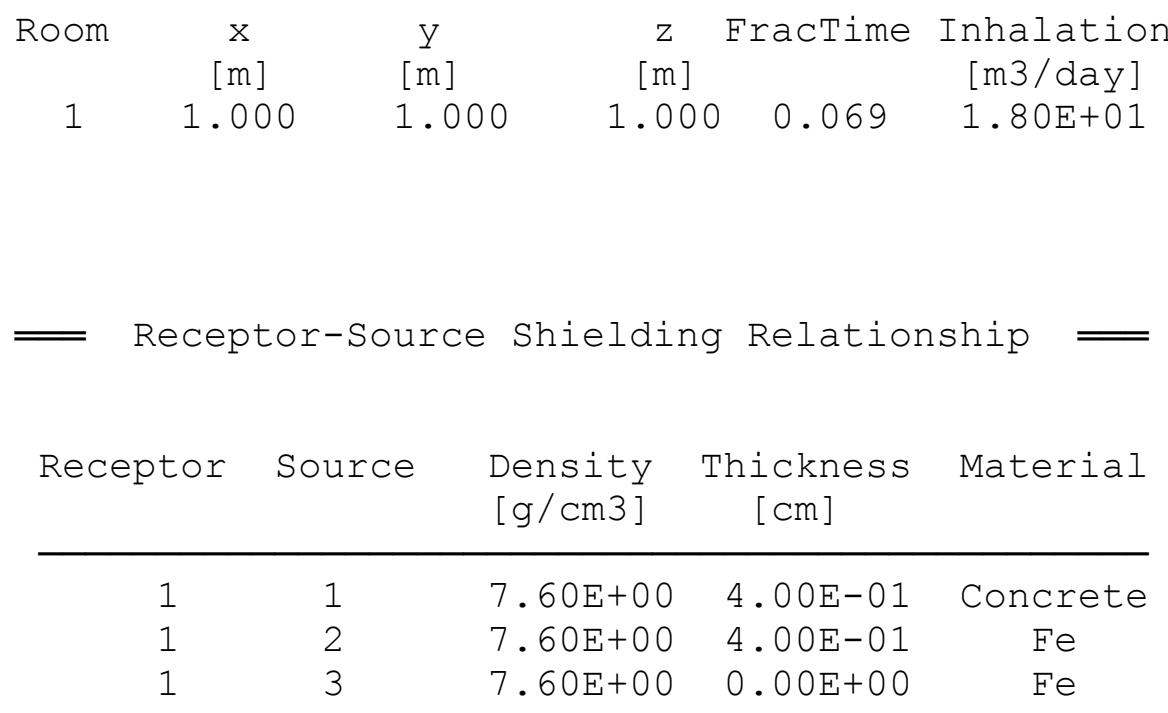


** RESRAD-BUILD Dose Program Output, Version 3.50 05/04/10 10:52:40 Page: 3 ** Title : Likely Use 2 - Employee

Input File : sitel.bld

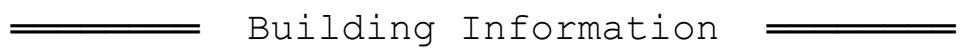

Building Air Exchange Rate: 8.00E-01 1/hr

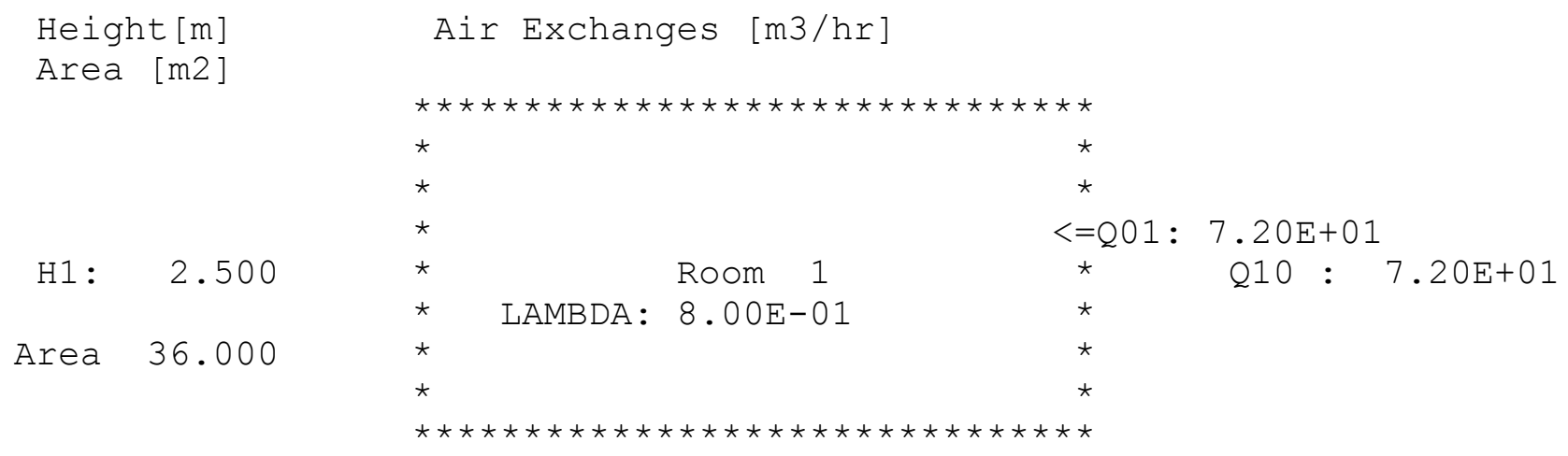

Deposition velocity: 1.00E-02 [m/s] Resuspension Rate: 5.00E-07 [1/s] 
RESRAD-BUILD Dose Program Output, Version 3.50 05/04/10 10:52:40 Page: 4 ** Title : Likely Use 2 - Employee

Input File : sitel.bld

$=$ Source Information

Source: 1

Location: : Room : $1 \mathrm{x}: 0.70 \mathrm{y}: 1.00 \mathrm{z}: 1.00[\mathrm{~m}]$

Geometry:: Type: Area Length $[\mathrm{m}]: 2.00 \mathrm{E}+00$ Width $[\mathrm{m}]: 2.00 \mathrm{E}+00$ Direction: $x$

Pathway : :

Direct Ingestion Rate: $0.000 \mathrm{E}+00$ [1/hr]

Fraction released to air: 1.000E-01

Removable fraction: $\quad 1.000 \mathrm{E}-02$

Time to Remove: $\quad 3.650 \mathrm{E}+02$ [day]

Contamination: :

Nuclide Concentration Dose Conversion Factor (Library: ICRP 72 (Adult))

\begin{tabular}{llllll}
\cline { 5 - 6 } \cline { 5 - 6 } & & & $\begin{array}{c}\text { Ingestion } \\
{[\text { mrem/dpm }]}\end{array}$ & $\begin{array}{c}\text { Inhalation } \\
{[\text { mrem/dpm }]}\end{array}$ & $\begin{array}{l}\text { Submersion } \\
{[\text { mrem/yr/ }} \\
(\text { dpm/m3) }]\end{array}$ \\
$\mathrm{U}-235$ & $8.000 \mathrm{E}+03$ & $7.890 \mathrm{E}-05$ & $1.417 \mathrm{E}-02$ & $4.063 \mathrm{E}-04$ \\
$\mathrm{PA}-231$ & $0.000 \mathrm{E}+00$ & $1.183 \mathrm{E}-03$ & $2.333 \mathrm{E}-01$ & $9.049 \mathrm{E}-05$ \\
$\mathrm{AC}-227$ & $0.000 \mathrm{E}+00$ & $2.015 \mathrm{E}-03$ & $9.476 \mathrm{E}-01$ & $9.734 \mathrm{E}-04$ \\
$\mathrm{CS}-137$ & $1.100 \mathrm{E}+06$ & $2.167 \mathrm{E}-05$ & $6.500 \mathrm{E}-05$ & $1.434 \mathrm{E}-03$ \\
$\mathrm{SR}-90$ & $1.100 \mathrm{E}+06$ & $5.117 \mathrm{E}-05$ & $2.692 \mathrm{E}-04$ & $1.039 \mathrm{E}-05$
\end{tabular}

Source: 2

Location: : Room : $1 \mathrm{x}: 1.30 \mathrm{y}: 1.00 \mathrm{z}: 1.00[\mathrm{~m}]$

Geometry:: Type: Area Length $[\mathrm{m}]: 2.00 \mathrm{E}+00$ Width $[\mathrm{m}]: 2.00 \mathrm{E}+00$ Direction: $x$

Pathway : :

Direct Ingestion Rate: $0.000 \mathrm{E}+00$ [1/hr]

Fraction released to air: 1.000E-01

Removable fraction: $\quad 1.000 \mathrm{E}-02$

Time to Remove: $\quad 3.650 \mathrm{E}+02$ [day]

Contamination: :

Nuclide Concentration Dose Conversion Factor (Library: ICRP 72 (Adult))

\begin{tabular}{|c|c|c|c|c|}
\hline & [dpm/m2 ] & $\begin{array}{l}\text { Ingestion } \\
\text { [mrem/dpm] }\end{array}$ & $\begin{array}{l}\text { Inhalation } \\
\text { [mrem/dpm ] }\end{array}$ & $\begin{array}{l}\text { Submersion } \\
{[\mathrm{mrem} / \mathrm{yr} /} \\
(\mathrm{dpm} / \mathrm{m} 3)]\end{array}$ \\
\hline$U-235$ & $8.000 E+03$ & $7.890 \mathrm{E}-05$ & $1.417 \mathrm{E}-02$ & $4.063 E-04$ \\
\hline
\end{tabular}


RESRAD-BUILD Dose Program Output, Version 3.50 05/04/10 10:52:40 Page: 5 ** Title : Likely Use 2 - Employee

Input File : sitel.bld

$\begin{array}{lllll}\mathrm{PA}-231 & 0.000 \mathrm{E}+00 & 1.183 \mathrm{E}-03 & 2.333 \mathrm{E}-01 & 9.049 \mathrm{E}-05 \\ \mathrm{AC}-227 & 0.000 \mathrm{E}+00 & 2.015 \mathrm{E}-03 & 9.476 \mathrm{E}-01 & 9.734 \mathrm{E}-04 \\ \mathrm{CS}-137 & 1.100 \mathrm{E}+06 & 2.167 \mathrm{E}-05 & 6.500 \mathrm{E}-05 & 1.434 \mathrm{E}-03 \\ \mathrm{SR}-90 & 1.100 \mathrm{E}+06 & 5.117 \mathrm{E}-05 & 2.692 \mathrm{E}-04 & 1.039 \mathrm{E}-05\end{array}$

Source: 3

Location: : Room : $1 \mathrm{x}: 1.00 \mathrm{y}: 1.30 \mathrm{z}: 1.00[\mathrm{~m}]$

Geometry:: Type: Area Length $[\mathrm{m}]: 2.00 \mathrm{E}+00$ Width $[\mathrm{m}]: 2.00 \mathrm{E}+00$ Direction: y

Pathway : :

Direct Ingestion Rate: $0.000 \mathrm{E}+00$ [1/hr]

Fraction released to air: 1.000E-01

Removable fraction: $\quad 1.000 \mathrm{E}-01$

Time to Remove: $\quad 3.650 \mathrm{E}+02$ [day]

Contamination: :

Nuclide Concentration Dose Conversion Factor (Library: ICRP 72 (Adult))

\begin{tabular}{|c|c|c|c|c|}
\hline & {$[\mathrm{dpm} / \mathrm{m} 2]$} & $\begin{array}{l}\text { Ingestion } \\
\text { [mrem/dpm] }\end{array}$ & $\begin{array}{l}\text { Inhalation } \\
\text { [mrem/dpm ] }\end{array}$ & $\begin{array}{l}\text { Submersion } \\
{[\mathrm{mrem} / \mathrm{yr} /} \\
(\mathrm{dpm} / \mathrm{m} 3)]\end{array}$ \\
\hline$U-235$ & $8.000 E+03$ & $7.890 E-05$ & $1.417 E-02$ & $4.063 E-04$ \\
\hline$P A-231$ & $0.000 E+00$ & $1.183 E-03$ & $2.333 E-01$ & $9.049 E-05$ \\
\hline$A C-227$ & $0.000 \mathrm{E}+00$ & $2.015 E-03$ & $9.476 E-01$ & $9.734 \mathrm{E}-04$ \\
\hline$C S-137$ & $1.500 \mathrm{E}+05$ & $2.167 \mathrm{E}-05$ & $6.500 E-05$ & $1.434 \mathrm{E}-03$ \\
\hline$S R-90$ & $1.500 \mathrm{E}+05$ & $5.117 \mathrm{E}-05$ & $2.692 \mathrm{E}-04$ & $1.039 \mathrm{E}-05$ \\
\hline
\end{tabular}


* RESRAD-BUILD Dose Program Output, Version 3.50 05/04/10 10:52:40 Page: 6 ** Title : Likely Use 2 - Employee

Input File : sitel.bld

Evaluation Time: $0.00000000 \mathrm{E}+00$ years

\begin{tabular}{rrrrr}
\hline \hline & & & & \\
\hline \hline & Assessment for Time: & 1 & $=$ \\
\hline \hline & Time & $=0.00 \mathrm{E}+00$ & yr & \\
\hline \hline
\end{tabular}

$=$ Source Information

Source: 1

Location: : Room : $1 \mathrm{x}: 0.70 \mathrm{y}: 1.00 \mathrm{z}: 1.00$ [m]

Geometry:: Type: Area Length $[\mathrm{m}]: 2.00 \mathrm{E}+00$ Width $[\mathrm{m}]: 2.00 \mathrm{E}+00$ Direction: $\mathrm{x}$

Pathway : :

Direct Ingestion Rate: $0.000 \mathrm{E}+00$ [1/hr]

Fraction released to air: 1.000E-01

Removable fraction: $\quad 1.000 \mathrm{E}-02$

Time to Remove: 3.650E+02 [day]

\begin{tabular}{|c|c|c|}
\hline \multirow[t]{6}{*}{ Contamination: : } & Nuclide & $\begin{array}{c}\text { Concentration } \\
{[\mathrm{dpm} / \mathrm{m} 2]}\end{array}$ \\
\hline & $U-235$ & $8.000 \mathrm{E}+03$ \\
\hline & $\mathrm{PA}-231$ & $0.000 \mathrm{E}+00$ \\
\hline & $A C-227$ & $0.000 \mathrm{E}+00$ \\
\hline & $C S-137$ & $1.100 \mathrm{E}+06$ \\
\hline & $S R-90$ & $1.100 \mathrm{E}+06$ \\
\hline
\end{tabular}

Source: 2

Location: : Room : $1 \mathrm{x}: 1.30 \mathrm{y}: 1.00 \mathrm{z}: 1.00[\mathrm{~m}]$

Geometry:: Type: Area Length $[\mathrm{m}]: 2.00 \mathrm{E}+00$ Width $[\mathrm{m}]: 2.00 \mathrm{E}+00$ Direction: $\mathrm{x}$

Pathway : :

Direct Ingestion Rate: $0.000 \mathrm{E}+00$ [1/hr]

Fraction released to air: 1.000E-01

Removable fraction: $\quad 1.000 \mathrm{E}-02$

Time to Remove: 3.650E+02 [day]

Contamination: : Nuclide Concentration

$\begin{array}{ll}\mathrm{U}-235 & 8.000 \mathrm{E}+03 \\ \mathrm{PA}-231 & 0.000 \mathrm{E}+00 \\ \mathrm{AC}-227 & 0.000 \mathrm{E}+00 \\ \mathrm{CS}-137 & 1.100 \mathrm{E}+06 \\ \mathrm{SR}-90 & 1.100 \mathrm{E}+06\end{array}$ 


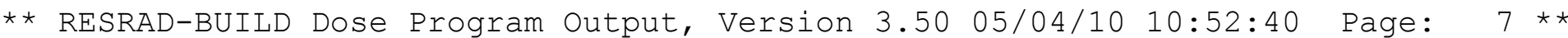
Title : Likely Use 2 - Employee

Input File : sitel.bld

Evaluation Time: $0.00000000 \mathrm{E}+00$ years

Source: 3

Location: : Room : $1 \mathrm{x}: 1.00 \mathrm{y}: 1.30 \mathrm{z}: 1.00$ [m]

Geometry:: Type: Area Length $[\mathrm{m}]: 2.00 \mathrm{E}+00$ Width $[\mathrm{m}]: 2.00 \mathrm{E}+00$ Direction: y

Pathway : :

Direct Ingestion Rate: $0.000 \mathrm{E}+00 \quad[1 / \mathrm{hr}]$

Fraction released to air: 1.000E-01

Removable fraction: $\quad 1.000 \mathrm{E}-01$

Time to Remove: $\quad 3.650 \mathrm{E}+02$ [day]

\begin{tabular}{|c|c|c|}
\hline \multirow[t]{6}{*}{ Contamination: : } & Nuclide & $\begin{array}{c}\text { Concentration } \\
{[\mathrm{dpm} / \mathrm{m} 2]}\end{array}$ \\
\hline & $U-235$ & $8.000 \mathrm{E}+03$ \\
\hline & $P A-231$ & $0.000 \mathrm{E}+00$ \\
\hline & $A C-227$ & $0.000 \mathrm{E}+00$ \\
\hline & $C S-137$ & $1.500 \mathrm{E}+05$ \\
\hline & $S R-90$ & $1.500 \mathrm{E}+05$ \\
\hline
\end{tabular}


** RESRAD-BUILD Dose Program Output, Version 3.50 05/04/10 10:52:40 Title : Likely Use 2 - Employee

Input File : sitel.bld

Evaluation Time: $0.00000000 \mathrm{E}+00$ years

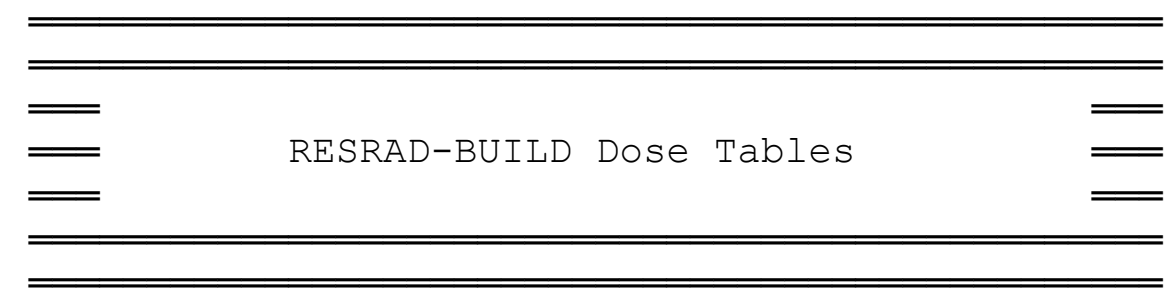

Source Contributions to Receptor Doses

=

[mrem]

Source Source Source Total

123

Receptor $1 \quad 2.41 E-01 \quad 2.31 E-01 \quad 4.02 E-02 \quad 5.12 E-01$

Total 2.41E-01 2.31E-01 4.02E-02 5.12E-01 
** RESRAD-BUILD Dose Program Output, Version 3.50 05/04/10 10:52:40 Title : Likely Use 2 - Employee

Input File : sitel.bld

Evaluation Time: $0.00000000 \mathrm{E}+00$ years

Pathway Detail of Doses

[mrem $]$

Source: 1

$\begin{array}{cllllll}\text { Receptor } & \text { External } & \text { Deposition } & \text { Immersion } & \text { Inhalation } & \text { Radon } & \text { Ingestion } \\ 1 & 2.40 \mathrm{E}-01 & 9.32 \mathrm{E}-05 & 3.29 \mathrm{E}-07 & 6.58 \mathrm{E}-04 & 0.00 \mathrm{E}+00 & 2.91 \mathrm{E}-04 \\ \text { Total } & 2.40 \mathrm{E}-01 & 9.32 \mathrm{E}-05 & 3.29 \mathrm{E}-07 & 6.58 \mathrm{E}-04 & 0.00 \mathrm{E}+00 & 2.91 \mathrm{E}-04\end{array}$

Source: 2

$\begin{array}{cllllll}\text { Receptor } & \text { External } & \text { Deposition } & \text { Immersion } & \text { Inhalation } & \text { Radon } & \text { Ingestion } \\ 1 & 2.29 \mathrm{E}-01 & 9.32 \mathrm{E}-05 & 3.29 \mathrm{E}-07 & 6.58 \mathrm{E}-04 & 0.00 \mathrm{E}+00 & 2.91 \mathrm{E}-04 \\ \text { Total } & 2.29 \mathrm{E}-01 & 9.32 \mathrm{E}-05 & 3.29 \mathrm{E}-07 & 6.58 \mathrm{E}-04 & 0.00 \mathrm{E}+00 & 2.91 \mathrm{E}-04\end{array}$

Source: 3

$\begin{array}{cllllll}\text { Receptor } & \text { External } & \text { Deposition } & \text { Immersion } & \text { Inhalation } & \text { Radon } & \text { Ingestion } \\ 1 & 3.74 \mathrm{E}-02 & 1.29 \mathrm{E}-04 & 4.55 \mathrm{E}-07 & 2.27 \mathrm{E}-03 & 0.00 \mathrm{E}+00 & 4.18 \mathrm{E}-04 \\ \text { Total } & 3.74 \mathrm{E}-02 & 1.29 \mathrm{E}-04 & 4.55 \mathrm{E}-07 & 2.27 \mathrm{E}-03 & 0.00 \mathrm{E}+00 & 4.18 \mathrm{E}-04\end{array}$


* RESRAD-BUILD Dose Program Output, Version 3.50 05/04/10 10:52:40 Page: 10 ** Title : Likely Use 2 - Employee

Input File : sitel.bld

Evaluation Time: $0.00000000 \mathrm{E}+00$ years

Nuclide Detail of Doses

[mrem ]

Source: 1

$\begin{array}{lcc}\text { Nuclide } & \begin{array}{c}\text { Receptor } \\ 1\end{array} & \text { Total } \\ \text { U-235 } & 7.08 \mathrm{E}-04 & 7.08 \mathrm{E}-04 \\ \mathrm{PA}-231 & 2.88 \mathrm{E}-08 & 2.88 \mathrm{E}-08 \\ \mathrm{AC}-227 & 1.24 \mathrm{E}-09 & 1.24 \mathrm{E}-09 \\ \mathrm{CS}-137 & 2.38 \mathrm{E}-01 & 2.38 \mathrm{E}-01 \\ \text { SR-90 } & 2.51 \mathrm{E}-03 & 2.51 \mathrm{E}-03\end{array}$

Source: 2

$\begin{array}{lcc}\text { Nuclide } & \text { Receptor } & \text { Total } \\ & 1 & \\ \text { U-235 } & 4.58 E-04 & 4.58 E-04 \\ \text { PA-231 } & 2.85 E-08 & 2.85 E-08 \\ \text { AC-227 } & 1.20 E-09 & 1.20 E-09 \\ \text { CS-137 } & 2.28 E-01 & 2.28 E-01 \\ \text { SR-90 } & 2.44 E-03 & 2.44 E-03\end{array}$

Source: 3

Nuclide Receptor Total

1

$\begin{array}{lll}\mathrm{U}-235 & 2.24 \mathrm{E}-03 \quad 2.24 \mathrm{E}-03\end{array}$

$\begin{array}{lll}\mathrm{PA}-231 & 2.77 \mathrm{E}-07 & 2.77 \mathrm{E}-07\end{array}$

$\mathrm{AC}-227 \quad 1.12 \mathrm{E}-08 \quad 1.12 \mathrm{E}-08$

$\begin{array}{lll}C S-137 & 3.68 \mathrm{E}-02 & 3.68 \mathrm{E}-02\end{array}$

SR-90 1.19E-03 1.19E-03 
* RESRAD-BUILD Dose Program Output, Version 3.50 05/04/10 10:52:40 Page: 11 ** Title : Likely Use 2 - Employee

Input File : sitel.bld

Evaluation Time: 1.00000000 years

\begin{tabular}{rrrrr}
\hline \hline & & & & \\
\hline \hline$=$ & Assessment for Time: & 2 & $=$ \\
\hline \hline & Time & $=1.00 \mathrm{E}+00$ & yr & \\
\hline \hline
\end{tabular}

$=$ Source Information

Source: 1

Location: : Room : $1 \mathrm{x}: 0.70 \mathrm{y}: 1.00 \mathrm{z}: 1.00$ [m]

Geometry:: Type: Area Length $[\mathrm{m}]: 2.00 \mathrm{E}+00$ Width $[\mathrm{m}]: 2.00 \mathrm{E}+00$ Direction: $\mathrm{x}$

Pathway : :

Direct Ingestion Rate: $0.000 \mathrm{E}+00$ [1/hr]

Fraction released to air: 1.000E-01

Removable fraction: $\quad 0.000 \mathrm{E}+00$

Time to Remove: 3.650E+02 [day]

\begin{tabular}{|c|c|c|}
\hline \multirow[t]{6}{*}{ Contamination: : } & Nuclide & $\begin{array}{c}\text { Concentration } \\
{[\mathrm{dpm} / \mathrm{m} 2]}\end{array}$ \\
\hline & $U-235$ & $7.920 \mathrm{E}+03$ \\
\hline & $\mathrm{PA}-231$ & $1.674 \mathrm{E}-01$ \\
\hline & $A C-227$ & $2.633 E-03$ \\
\hline & $C S-137$ & $1.064 \mathrm{E}+06$ \\
\hline & $S R-90$ & $1.063 E+06$ \\
\hline
\end{tabular}

Source: 2

Location: : Room : $1 \mathrm{x}: 1.30 \mathrm{y}: 1.00 \mathrm{z}: 1.00[\mathrm{~m}]$

Geometry:: Type: Area Length $[\mathrm{m}]: 2.00 \mathrm{E}+00$ Width $[\mathrm{m}]: 2.00 \mathrm{E}+00$ Direction: $\mathrm{x}$

Pathway : :

Direct Ingestion Rate: $0.000 \mathrm{E}+00 \quad[1 / \mathrm{hr}]$

Fraction released to air: 1.000E-01

Removable fraction: $\quad 0.000 \mathrm{E}+00$

Time to Remove: $\quad 3.650 \mathrm{E}+02$ [day]

Contamination: : Nuclide Concentration

$\begin{array}{ll}\mathrm{U}-235 & 7.920 \mathrm{E}+03 \\ \mathrm{PA}-231 & 1.674 \mathrm{E}-01 \\ \mathrm{AC}-227 & 2.633 \mathrm{E}-03 \\ \mathrm{CS}-137 & 1.064 \mathrm{E}+06 \\ \mathrm{SR}-90 & 1.063 \mathrm{E}+06\end{array}$ 
* RESRAD-BUILD Dose Program Output, Version 3.50 05/04/10 10:52:40 Page: 12 ** Title : Likely Use 2 - Employee

Input File : sitel.bld

Evaluation Time: 1.00000000 years

Source: 3

Location: : Room : $1 \mathrm{x}: 1.00 \mathrm{y}: 1.30 \mathrm{z}: 1.00$ [m]

Geometry:: Type: Area Length $[\mathrm{m}]: 2.00 \mathrm{E}+00$ Width $[\mathrm{m}]: 2.00 \mathrm{E}+00$ Direction: y

Pathway : :

$\begin{array}{lll}\text { Direct Ingestion Rate: } & 0.000 \mathrm{E}+00 & {[1 / \mathrm{hr}]} \\ \text { Fraction released to air: } & 1.000 \mathrm{E}-01 & \\ \text { Removable fraction: } & 0.000 \mathrm{E}+00 & \\ \text { Time to Remove: } & 3.650 \mathrm{E}+02 & \text { [day] }\end{array}$

Contamination: : Nuclide

Concentration

[dpm/m2 ]

$\mathrm{U}-235 \quad 7.200 \mathrm{E}+03$

$\mathrm{PA}-231 \quad 1.522 \mathrm{E}-01$

AC-227 2.393E-03

CS-137 1.319E+05

SR-90 1.318E+05 
** RESRAD-BUILD Dose Program Output, Version 3.50 05/04/10 10:52:40 Title : Likely Use 2 - Employee

Input File : sitel.bld

Evaluation Time: 1.00000000 years

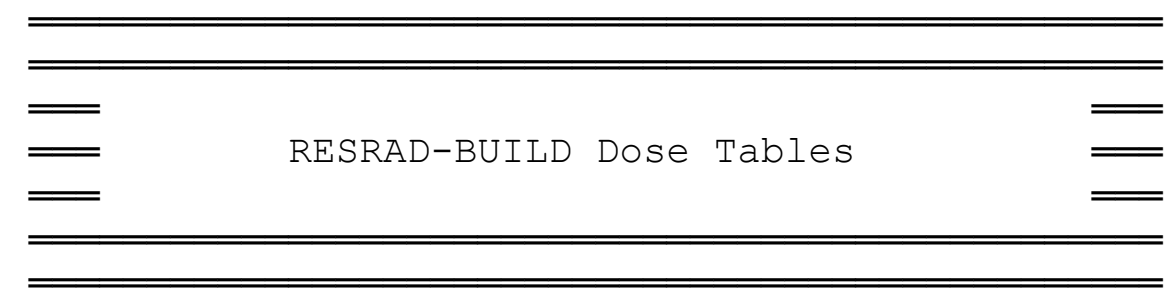

Source Contributions to Receptor Doses

ב

[mrem]

Source Source Source Total

$\begin{array}{llll}1 & 2 & 3\end{array}$

Receptor $1 \quad 2.33 E-01 \quad 2.23 E-01 \quad 3.46 E-02 \quad 4.91 E-01$

Total 2.33E-01 2.23E-01 3.46E-02 $4.91 \mathrm{E}-01$ 
* RESRAD-BUILD Dose Program Output, Version 3.50 05/04/10 10:52:40 Page: 14 ** Title : Likely Use 2 - Employee

Input File : sitel.bld

Evaluation Time: 1.00000000 years

Pathway Detail of Doses

[mrem $]$

Source: 1

Receptor

1

Total

External

Deposition

Immersion

Inhalation

Radon

Ingestion

2.33E-01

$0.00 \mathrm{E}+00$

$0.00 \mathrm{E}+00$

$0.00 \mathrm{E}+00$

$0.00 \mathrm{E}+00$

$0.00 E+00$

2. 33E-01

$0.00 \mathrm{E}+00$

$0.00 \mathrm{E}+00$

$0.00 \mathrm{E}+00$

$0.00 \mathrm{E}+00$

$0.00 \mathrm{E}+00$

Source: 2

Receptor 1

Total

External

Deposition

Immersion

Inhalation

Radon

2.23E-01

$0.00 \mathrm{E}+00$

$0.00 \mathrm{E}+00$

$0.00 \mathrm{E}+00$

$0.00 \mathrm{E}+00$

$0.00 \mathrm{E}+00$

$0.00 \mathrm{E}+00$

Ingestion

$0.00 \mathrm{E}+00$

$0.00 \mathrm{E}+00$

$0.00 \mathrm{E}+00$

$0.00 \mathrm{E}+00$

Source: 3

Receptor 1

External

Deposition

Immersion

Inhalation

Radon

3. 46E-02

Total

3. $46 \mathrm{E}-02$

$0.00 \mathrm{E}+00$

$0.00 \mathrm{E}+00$

$0.00 \mathrm{E}+00$

$0.00 \mathrm{E}+00$

$0.00 \mathrm{E}+00$

$0.00 \mathrm{E}+00$

Ingestion

$0.00 \mathrm{E}+00$

$0.00 \mathrm{E}+00$ 
* RESRAD-BUILD Dose Program Output, Version 3.50 05/04/10 10:52:40 Page: 15 ** Title : Likely Use 2 - Employee

Input File : sitel.bld

Evaluation Time: 1.00000000 years

Nuclide Detail of Doses

[mrem ]

Source: 1

$\begin{array}{lcc}\text { Nuclide } & \begin{array}{c}\text { Receptor } \\ 1\end{array} & \text { Total } \\ \text { U-235 } & 5.43 E-04 & 5.43 E-04 \\ \text { PA-231 } & 3.74 \mathrm{E}-09 & 3.74 \mathrm{E}-09 \\ \mathrm{AC}-227 & 9.71 \mathrm{E}-10 & 9.71 \mathrm{E}-10 \\ \mathrm{CS}-137 & 2.31 \mathrm{E}-01 & 2.31 \mathrm{E}-01 \\ \mathrm{SR}-90 & 1.85 \mathrm{E}-03 & 1.85 \mathrm{E}-03\end{array}$

Source: 2

Nuclide Receptor Total

1

$\begin{array}{lll}\mathrm{U}-235 & 2.94 \mathrm{E}-04 & 2.94 \mathrm{E}-04\end{array}$

$\mathrm{PA}-231 \quad 3.01 \mathrm{E}-09 \quad 3.01 \mathrm{E}-09$

$\mathrm{AC}-227 \quad 6.94 \mathrm{E}-10 \quad 6.94 \mathrm{E}-10$

$\begin{array}{lll}C S-137 & 2.21 \mathrm{E}-01 & 2.21 \mathrm{E}-01\end{array}$

SR-90 1.78E-03 1.78E-03

Source: 3

$\begin{array}{lcc}\text { Nuclide } & \begin{array}{c}\text { Receptor } \\ 1\end{array} & \text { Total } \\ \text { U-235 } & 5.90 E-04 & 5.90 E-04 \\ \text { PA-231 } & 5.34 E-09 & 5.34 E-09 \\ \text { AC-227 } & 1.01 E-09 & 1.01 E-09 \\ \text { CS-137 } & 3.37 E-02 & 3.37 E-02 \\ \text { SR-90 } & 3.41 E-04 & 3.41 E-04\end{array}$


* RESRAD-BUILD Dose Program Output, Version 3.50 05/04/10 10:52:40 Page: 16 ** Title : Likely Use 2 - Employee

Input File : sitel.bld

Evaluation Time: 10.0000000 years

\begin{tabular}{rrrrr}
\hline \hline & & & & \\
\hline \hline & Assessment for Time: & 3 & $=$ \\
\hline \hline & Time & $=1.00 \mathrm{E}+01$ & yr & \\
\hline \hline
\end{tabular}

$=$ Source Information

Source: 1

Location: : Room : $1 \mathrm{x}: 0.70 \mathrm{y}: 1.00 \mathrm{z}: 1.00$ [m]

Geometry:: Type: Area Length $[\mathrm{m}]: 2.00 \mathrm{E}+00$ Width $[\mathrm{m}]: 2.00 \mathrm{E}+00$ Direction: $\mathrm{x}$

Pathway : :

Direct Ingestion Rate: $0.000 \mathrm{E}+00$ [1/hr]

Fraction released to air: 1.000E-01

Removable fraction: $\quad 0.000 \mathrm{E}+00$

Time to Remove: 3.650E+02 [day]

\begin{tabular}{|c|c|c|}
\hline \multirow[t]{6}{*}{ Contamination: : } & Nuclide & $\begin{array}{c}\text { Concentration } \\
{[\mathrm{dpm} / \mathrm{m} 2]}\end{array}$ \\
\hline & $U-235$ & $7.920 E+03$ \\
\hline & $\mathrm{PA}-231$ & $1.674 \mathrm{E}+00$ \\
\hline & $A C-227$ & $2.400 \mathrm{E}-01$ \\
\hline & $C S-137$ & $8.643 E+05$ \\
\hline & $S R-90$ & $8.582 E+05$ \\
\hline
\end{tabular}

Source: 2

Location: : Room : $1 \mathrm{x}: 1.30 \mathrm{y}: 1.00 \mathrm{z}: 1.00[\mathrm{~m}]$

Geometry:: Type: Area Length $[\mathrm{m}]: 2.00 \mathrm{E}+00$ Width $[\mathrm{m}]: 2.00 \mathrm{E}+00$ Direction: $\mathrm{x}$

Pathway : :

Direct Ingestion Rate: $0.000 \mathrm{E}+00 \quad[1 / \mathrm{hr}]$

Fraction released to air: 1.000E-01

Removable fraction: $\quad 0.000 \mathrm{E}+00$

Time to Remove: $\quad 3.650 \mathrm{E}+02$ [day]

Contamination: : Nuclide Concentration

$\begin{array}{ll}\mathrm{U}-235 & 7.920 \mathrm{E}+03 \\ \mathrm{PA}-231 & 1.674 \mathrm{E}+00 \\ \mathrm{AC}-227 & 2.400 \mathrm{E}-01 \\ \mathrm{CS}-137 & 8.643 \mathrm{E}+05 \\ \mathrm{SR}-90 & 8.582 \mathrm{E}+05\end{array}$ 
* RESRAD-BUILD Dose Program Output, Version 3.50 05/04/10 10:52:40 Page: 17 ** Title : Likely Use 2 - Employee

Input File : sitel.bld

Evaluation Time: 10.0000000 years

Source: 3

Location: : Room : $1 \mathrm{x}: 1.00 \mathrm{y}: 1.30 \mathrm{z}: 1.00$ [m]

Geometry:: Type: Area Length $[\mathrm{m}]: 2.00 \mathrm{E}+00$ Width $[\mathrm{m}]: 2.00 \mathrm{E}+00$ Direction: y

Pathway : :

Direct Ingestion Rate: $0.000 \mathrm{E}+00$ [1/hr]

Fraction released to air: 1.000E-01

Removable fraction: $\quad 0.000 \mathrm{E}+00$

Time to Remove: $\quad 3.650 \mathrm{E}+02$ [day]

\begin{tabular}{|c|c|c|}
\hline \multirow[t]{6}{*}{ Contamination: : } & Nuclide & $\begin{array}{c}\text { Concentration } \\
{[\mathrm{dom} / \mathrm{m} 2]}\end{array}$ \\
\hline & $U-235$ & $7.200 E+03$ \\
\hline & $P A-231$ & $1.521 \mathrm{E}+00$ \\
\hline & $A C-227$ & $2.182 \mathrm{E}-01$ \\
\hline & CS-137 & $1.071 E+05$ \\
\hline & $S R-90$ & $1.064 E+05$ \\
\hline
\end{tabular}


** RESRAD-BUILD Dose Program Output, Version 3.50 05/04/10 10:52:40 Title : Likely Use 2 - Employee

Input File : sitel.bld

Evaluation Time: 10.0000000 years

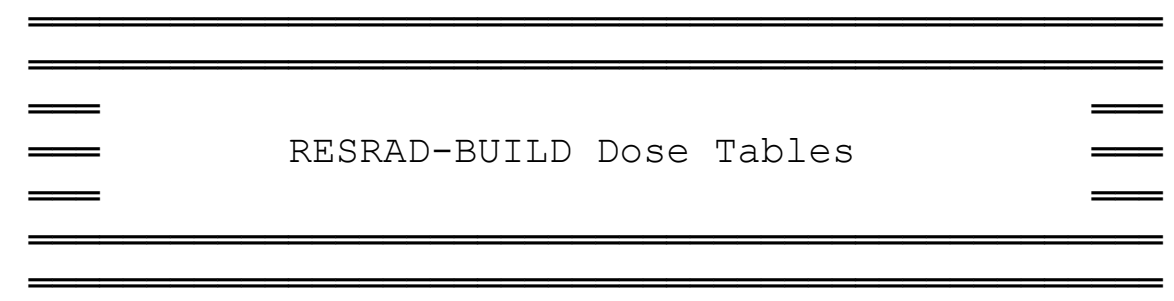

Source Contributions to Receptor Doses

ב

[mrem]

Source Source Source Total

$\begin{array}{llll}1 & 2 & 3\end{array}$

Receptor $1 \quad 1.90 \mathrm{E}-01 \quad 1.81 \mathrm{E}-01 \quad 2.82 \mathrm{E}-02 \quad 3.99 \mathrm{E}-01$

Total 1.90E-01 1.81E-01 2.82E-02 $3.99 \mathrm{E}-01$ 
** RESRAD-BUILD Dose Program Output, Version 3.50 05/04/10 10:52:40 Title : Likely Use 2 - Employee

Input File : sitel.bld

Evaluation Time: 10.0000000 years

Pathway Detail of Doses

[mrem $]$

Source: 1

$\begin{array}{cllllll}\text { Receptor } & \text { External } & \text { Deposition } & \text { Immersion } & \text { Inhalation } & \text { Radon } & \text { Ingestion } \\ 1 & 1.90 \mathrm{E}-01 & 0.00 \mathrm{E}+00 & 0.00 \mathrm{E}+00 & 0.00 \mathrm{E}+00 & 0.00 \mathrm{E}+00 & 0.00 \mathrm{E}+00 \\ \text { Total } & 1.90 \mathrm{E}-01 & 0.00 \mathrm{E}+00 & 0.00 \mathrm{E}+00 & 0.00 \mathrm{E}+00 & 0.00 \mathrm{E}+00 & 0.00 \mathrm{E}+00\end{array}$

Source: 2

$\begin{array}{cllllll}\text { Receptor } & \text { External } & \text { Deposition } & \text { Immersion } & \text { Inhalation } & \text { Radon } & \text { Ingestion } \\ 1 & 1.81 \mathrm{E}-01 & 0.00 \mathrm{E}+00 & 0.00 \mathrm{E}+00 & 0.00 \mathrm{E}+00 & 0.00 \mathrm{E}+00 & 0.00 \mathrm{E}+00 \\ \text { Total } & 1.81 \mathrm{E}-01 & 0.00 \mathrm{E}+00 & 0.00 \mathrm{E}+00 & 0.00 \mathrm{E}+00 & 0.00 \mathrm{E}+00 & 0.00 \mathrm{E}+00\end{array}$

Source: 3

$\begin{array}{cllllll}\text { Receptor } & \text { External } & \text { Deposition } & \text { Immersion } & \text { Inhalation } & \text { Radon } & \text { Ingestion } \\ 1 & 2.82 \mathrm{E}-02 & 0.00 \mathrm{E}+00 & 0.00 \mathrm{E}+00 & 0.00 \mathrm{E}+00 & 0.00 \mathrm{E}+00 & 0.00 \mathrm{E}+00 \\ \text { Total } & 2.82 \mathrm{E}-02 & 0.00 \mathrm{E}+00 & 0.00 \mathrm{E}+00 & 0.00 \mathrm{E}+00 & 0.00 \mathrm{E}+00 & 0.00 \mathrm{E}+00\end{array}$


* RESRAD-BUILD Dose Program Output, Version 3.50 05/04/10 10:52:40 Page: 20 ** Title : Likely Use 2 - Employee

Input File : sitel.bld

Evaluation Time: 10.0000000 years

Nuclide Detail of Doses

[mrem ]

Source: 1

$\begin{array}{lcc}\text { Nuclide } & \begin{array}{c}\text { Receptor } \\ 1\end{array} & \text { Total } \\ \text { U-235 } & 5.43 \mathrm{E}-04 & 5.43 \mathrm{E}-04 \\ \mathrm{PA}-231 & 2.62 \mathrm{E}-08 & 2.62 \mathrm{E}-08 \\ \mathrm{AC}-227 & 4.19 \mathrm{E}-08 & 4.19 \mathrm{E}-08 \\ \mathrm{CS}-137 & 1.88 \mathrm{E}-01 & 1.88 \mathrm{E}-01 \\ \mathrm{SR}-90 & 1.49 \mathrm{E}-03 & 1.49 \mathrm{E}-03\end{array}$

Source: 2

Nuclide Receptor Total

1

U-235 2.94E-04 2.94E-04

$\mathrm{PA}-231 \quad 2.11 \mathrm{E}-08 \quad 2.11 \mathrm{E}-08$

$\begin{array}{lll}\mathrm{AC}-227 & 3.00 \mathrm{E}-08 & 3.00 \mathrm{E}-08\end{array}$

$\begin{array}{lll}\mathrm{CS}-137 & 1.80 \mathrm{E}-01 & 1.80 \mathrm{E}-01\end{array}$

SR-90 1.44E-03 1.44E-03

Source: 3

$\begin{array}{lcc}\text { Nuclide } & \begin{array}{c}\text { Receptor } \\ 1\end{array} & \text { Total } \\ \text { U-235 } & 5.90 \mathrm{E}-04 & 5.90 \mathrm{E}-04 \\ \mathrm{PA}-231 & 3.74 \mathrm{E}-08 & 3.74 \mathrm{E}-08 \\ \mathrm{AC}-227 & 4.36 \mathrm{E}-08 & 4.36 \mathrm{E}-08 \\ \mathrm{CS}-137 & 2.74 \mathrm{E}-02 & 2.74 \mathrm{E}-02 \\ \text { SR-90 } & 2.76 \mathrm{E}-04 & 2.76 \mathrm{E}-04\end{array}$


* RESRAD-BUILD Dose Program Output, Version 3.50 05/04/10 10:52:40 Page: 21 ** Title : Likely Use 2 - Employee

Input File : sitel.bld

Evaluation Time: 30.0000000 years

\begin{tabular}{rrrrr}
\hline \hline & & & & \\
\hline \hline$=$ & Assessment for Time: & 4 & $=$ \\
\hline \hline & Time & $=3.00 \mathrm{E}+01$ & yr & $=$ \\
\hline \hline
\end{tabular}

$=$ Source Information

Source: 1

Location: : Room : $1 \mathrm{x}: 0.70 \mathrm{y}: 1.00 \mathrm{z}: 1.00$ [m]

Geometry:: Type: Area Length $[\mathrm{m}]: 2.00 \mathrm{E}+00$ Width $[\mathrm{m}]: 2.00 \mathrm{E}+00$ Direction: $\mathrm{x}$

Pathway : :

Direct Ingestion Rate: $0.000 \mathrm{E}+00$ [1/hr]

Fraction released to air: 1.000E-01

Removable fraction: $\quad 0.000 \mathrm{E}+00$

Time to Remove: 3.650E+02 [day]

\begin{tabular}{|c|c|c|}
\hline \multirow[t]{6}{*}{ Contamination: : } & Nuclide & $\begin{array}{c}\text { Concentration } \\
{[\mathrm{dpm} / \mathrm{m} 2]}\end{array}$ \\
\hline & $U-235$ & $7.920 E+03$ \\
\hline & $\mathrm{PA}-231$ & $5.019 \mathrm{E}+00$ \\
\hline & $A C-227$ & $1.785 \mathrm{E}+00$ \\
\hline & $C S-137$ & $5.445 E+05$ \\
\hline & $S R-90$ & $5.330 E+05$ \\
\hline
\end{tabular}

Source: 2

Location: : Room : $1 \mathrm{x}: 1.30 \mathrm{y}: 1.00 \mathrm{z}: 1.00[\mathrm{~m}]$

Geometry:: Type: Area Length $[\mathrm{m}]: 2.00 \mathrm{E}+00$ Width $[\mathrm{m}]: 2.00 \mathrm{E}+00$ Direction: $\mathrm{x}$

Pathway : :

Direct Ingestion Rate: $0.000 \mathrm{E}+00 \quad[1 / \mathrm{hr}]$

Fraction released to air: 1.000E-01

Removable fraction: $\quad 0.000 \mathrm{E}+00$

Time to Remove: $\quad 3.650 \mathrm{E}+02$ [day]

Contamination: : Nuclide Concentration

$\begin{array}{ll}\mathrm{U}-235 & 7.920 \mathrm{E}+03 \\ \mathrm{PA}-231 & 5.019 \mathrm{E}+00 \\ \mathrm{AC}-227 & 1.785 \mathrm{E}+00 \\ \mathrm{CS}-137 & 5.445 \mathrm{E}+05 \\ \mathrm{SR}-90 & 5.330 \mathrm{E}+05\end{array}$ 
* RESRAD-BUILD Dose Program Output, Version 3.50 05/04/10 10:52:40 Page: 22 ** Title : Likely Use 2 - Employee

Input File : sitel.bld

Evaluation Time: 30.0000000 years

Source: 3

Location: : Room : $1 \mathrm{x}: 1.00 \mathrm{y}: 1.30 \mathrm{z}: 1.00$ [m]

Geometry:: Type: Area Length $[\mathrm{m}]: 2.00 \mathrm{E}+00$ Width $[\mathrm{m}]: 2.00 \mathrm{E}+00$ Direction: y

Pathway : :

$\begin{array}{lll}\text { Direct Ingestion Rate: } & 0.000 \mathrm{E}+00 & {[1 / \mathrm{hr}]} \\ \text { Fraction released to air: } & 1.000 \mathrm{E}-01 & \\ \text { Removable fraction: } & 0.000 \mathrm{E}+00 \\ \text { Time to Remove: } & 3.650 \mathrm{E}+02 \text { [day] }\end{array}$

Contamination: : Nuclide

Concentration

[dpm/m2 ]

$\mathrm{U}-235 \quad 7.200 \mathrm{E}+03$

$\mathrm{PA}-231 \quad 4.563 \mathrm{E}+00$

$\mathrm{AC}-227 \quad 1.622 \mathrm{E}+00$

CS-137 6.750E+04

SR-90 6.607E+04 
** RESRAD-BUILD Dose Program Output, Version 3.50 05/04/10 10:52:40 Title : Likely Use 2 - Employee

Input File : sitel.bld

Evaluation Time: 30.0000000 years

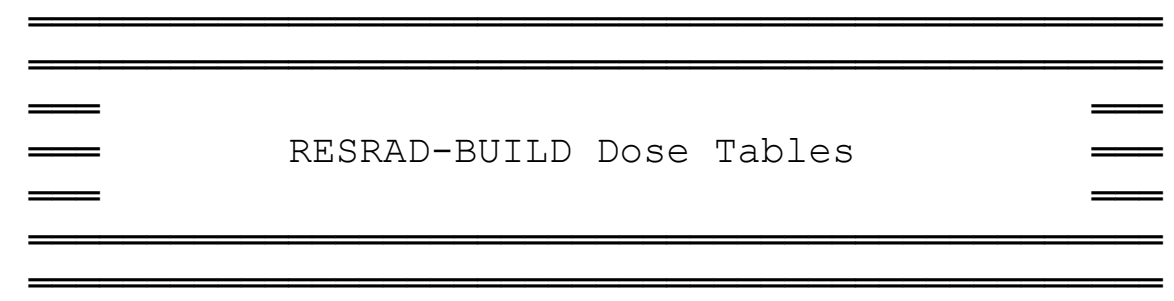

Source Contributions to Receptor Doses

ב

[mrem]

Source Source Source Total

123

Receptor $1 \quad 1.20 \mathrm{E}-01$ 1.14E-01 $1.80 \mathrm{E}-02 \quad 2.52 \mathrm{E}-01$

Total 1.20E-01 1.14E-01 1.80E-02 2.52E-01 
** RESRAD-BUILD Dose Program Output, Version 3.50 05/04/10 10:52:40 Title : Likely Use 2 - Employee

Input File : sitel.bld

Evaluation Time: 30.0000000 years

Pathway Detail of Doses

[mrem $]$

Source: 1

$\begin{array}{cllllll}\text { Receptor } & \text { External } & \text { Deposition } & \text { Immersion } & \text { Inhalation } & \text { Radon } & \text { Ingestion } \\ 1 & 1.20 \mathrm{E}-01 & 0.00 \mathrm{E}+00 & 0.00 \mathrm{E}+00 & 0.00 \mathrm{E}+00 & 0.00 \mathrm{E}+00 & 0.00 \mathrm{E}+00 \\ \text { Total } & 1.20 \mathrm{E}-01 & 0.00 \mathrm{E}+00 & 0.00 \mathrm{E}+00 & 0.00 \mathrm{E}+00 & 0.00 \mathrm{E}+00 & 0.00 \mathrm{E}+00\end{array}$

Source: 2

$\begin{array}{cllllll}\text { Receptor } & \text { External } & \text { Deposition } & \text { Immersion } & \text { Inhalation } & \text { Radon } & \text { Ingestion } \\ 1 & 1.14 \mathrm{E}-01 & 0.00 \mathrm{E}+00 & 0.00 \mathrm{E}+00 & 0.00 \mathrm{E}+00 & 0.00 \mathrm{E}+00 & 0.00 \mathrm{E}+00 \\ \text { Total } & 1.14 \mathrm{E}-01 & 0.00 \mathrm{E}+00 & 0.00 \mathrm{E}+00 & 0.00 \mathrm{E}+00 & 0.00 \mathrm{E}+00 & 0.00 \mathrm{E}+00\end{array}$

Source: 3

$\begin{array}{cllllll}\text { Receptor } & \text { External } & \text { Deposition } & \text { Immersion } & \text { Inhalation } & \text { Radon } & \text { Ingestion } \\ 1 & 1.80 \mathrm{E}-02 & 0.00 \mathrm{E}+00 & 0.00 \mathrm{E}+00 & 0.00 \mathrm{E}+00 & 0.00 \mathrm{E}+00 & 0.00 \mathrm{E}+00 \\ \text { Total } & 1.80 \mathrm{E}-02 & 0.00 \mathrm{E}+00 & 0.00 \mathrm{E}+00 & 0.00 \mathrm{E}+00 & 0.00 \mathrm{E}+00 & 0.00 \mathrm{E}+00\end{array}$


* RESRAD-BUILD Dose Program Output, Version 3.50 05/04/10 10:52:40 Page: 25 ** Title : Likely Use 2 - Employee

Input File : sitel.bld

Evaluation Time: 30.0000000 years

Nuclide Detail of Doses

[mrem ]

Source: 1

$\begin{array}{lcc}\text { Nuclide } & \begin{array}{c}\text { Receptor } \\ 1\end{array} & \text { Total } \\ \text { U-235 } & 5.43 \mathrm{E}-04 & 5.43 \mathrm{E}-04 \\ \mathrm{PA}-231 & 7.61 \mathrm{E}-08 & 7.61 \mathrm{E}-08 \\ \mathrm{AC}-227 & 2.92 \mathrm{E}-07 & 2.92 \mathrm{E}-07 \\ \mathrm{CS}-137 & 1.18 \mathrm{E}-01 & 1.18 \mathrm{E}-01 \\ \mathrm{SR}-90 & 9.27 \mathrm{E}-04 & 9.27 \mathrm{E}-04\end{array}$

Source: 2

Nuclide Receptor Total

1

$\begin{array}{lll}\mathrm{U}-235 & 2.94 \mathrm{E}-04 & 2.94 \mathrm{E}-04 \\ \mathrm{PA}-231 & 6.12 \mathrm{E}-08 & 6.12 \mathrm{E}-08 \\ \mathrm{AC}-227 & 2.09 \mathrm{E}-07 & 2.09 \mathrm{E}-07 \\ \mathrm{CS}-137 & 1.13 \mathrm{E}-01 & 1.13 \mathrm{E}-01 \\ \mathrm{SR}-90 & 8.92 \mathrm{E}-04 & 8.92 \mathrm{E}-04\end{array}$

Source: 3

Nuclide Receptor Total

1

$\begin{array}{lll}\mathrm{U}-235 & 5.90 \mathrm{E}-04 & 5.90 \mathrm{E}-04\end{array}$

$\begin{array}{lll}\mathrm{PA}-231 & 1.09 \mathrm{E}-07 & 1.09 \mathrm{E}-07\end{array}$

$\begin{array}{lll}\mathrm{AC}-227 & 3.04 \mathrm{E}-07 \quad 3.04 \mathrm{E}-07\end{array}$

$\begin{array}{lll}\mathrm{CS}-137 & 1.72 \mathrm{E}-02 & 1.72 \mathrm{E}-02\end{array}$

SR-90 1.71E-04 1.71E-04 
* RESRAD-BUILD Dose Program Output, Version 3.50 05/04/10 10:52:40 Page: 26 ** Title : Likely Use 2 - Employee

Input File : sitel.bld

Full Summary

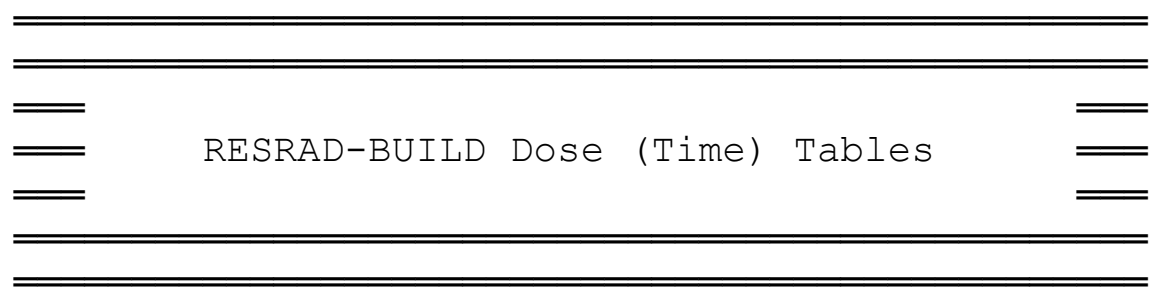

Receptor Dose Received for the Exposure Duration

=

(mrem)

Evaluation Time [yr]

$0.00 \mathrm{E}+00 \quad 1.00 \mathrm{E}+00 \quad 1.00 \mathrm{E}+01 \quad 3.00 \mathrm{E}+01$

$1 \quad 5.12 \mathrm{E}-01 \quad 4.91 \mathrm{E}-01 \quad 3.99 \mathrm{E}-01 \quad 2.52 \mathrm{E}-01$

Receptor Dose/Yr Averaged Over Exposure Duration

$(\mathrm{mrem} / \mathrm{yr})$

Evaluation Time [yr]

$0.00 \mathrm{E}+00 \quad 1.00 \mathrm{E}+00 \quad 1.00 \mathrm{E}+01 \quad 3.00 \mathrm{E}+01$

$15.12 \mathrm{E}-01 \quad 4.91 \mathrm{E}-01 \quad 3.99 \mathrm{E}-01 \quad 2.52 \mathrm{E}-01$ 
* RESRAD-BUILD Dose Program Output, Version 3.50 05/03/10 15:05:25 Page: 1 ** Title : Worst Case 1

Input File : sitel.bld

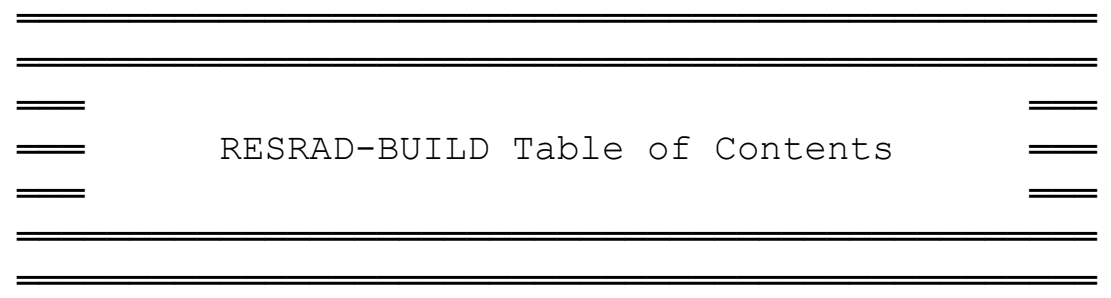

RESRAD-BUILD Input Parameters.......... 2

Building Information................ 3

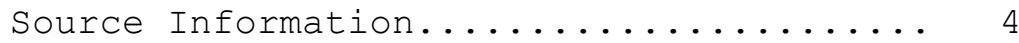

For time $=0.00 \mathrm{E}+00 \mathrm{yr}$

Time Specific Parameters........... 8

Receptor-Source Dose Summary............ 11

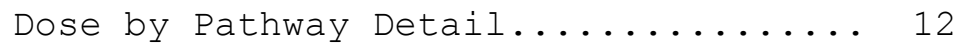

Dose by Nuclide Detail............ 13

For time $=1.00 \mathrm{E}+00 \mathrm{yr}$

Time Specific Parameters........... 15

Receptor-Source Dose Summary......... 18

Dose by Pathway Detail............. 19

Dose by Nuclide Detail........... 20

For time $=1.00 \mathrm{E}+01 \mathrm{yr}$

Time Specific Parameters.......... 22

Receptor-Source Dose Summary........ 25

Dose by Pathway Detail............ 26

Dose by Nuclide Detail........... 27

For time $=3.00 \mathrm{E}+01 \mathrm{yr}$

Time Specific Parameters.......... 29

Receptor-Source Dose Summary........ 32

Dose by Pathway Detail........... 33

Dose by Nuclide Detail........... 34

Full summary................ 36 
** RESRAD-BUILD Dose Program Output, Version 3.50 05/03/10 15:05:25 Page: 2 ** Title : Worst Case 1

Input File : sitel.bld

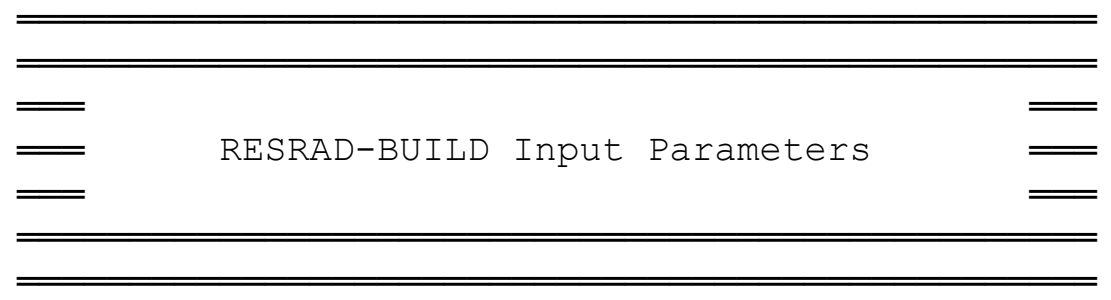

$\begin{array}{lrc}\text { Number of Sources }: & 6 \\ \text { Number of Receptors: } & 1 \\ \text { Total Time } & 3.650000 \mathrm{E}+02 \text { days } \\ \text { Fraction Inside }: & 5.000000 \mathrm{E}-01\end{array}$

$=$ Receptor Information $=$

Receptor

1

$\begin{array}{ccc}\text { Room } & \mathrm{x} & \mathrm{y} \\ & {[\mathrm{m}]} & {[\mathrm{m}]} \\ 1 & 0.915 & 2.500\end{array}$

$1 \quad 0.915$

2.500 z Fractime

$[\mathrm{m}]$

0.600

0.670

$[\mathrm{m} 3 / \mathrm{day}]$
$1.80 \mathrm{E}+01$

Ingestion (Dust)

[m2/hr]

$1.00 \mathrm{E}-04$

\begin{tabular}{ccccc}
\hline Receptor-Source Shielding Relationship & $=$ \\
Receptor & Source & $\begin{array}{c}\text { Density } \\
{[\mathrm{g} / \mathrm{cm} 3]}\end{array}$ & $\begin{array}{c}\text { Thickness } \\
{[\mathrm{cm}]}\end{array}$ & Material \\
\hline 1 & 1 & $7.60 \mathrm{E}+00$ & $0.00 \mathrm{E}+00$ & Concrete \\
1 & 2 & $7.60 \mathrm{E}+00$ & $1.00 \mathrm{E}-01$ & $\mathrm{Fe}$ \\
1 & 3 & $7.60 \mathrm{E}+00$ & $1.00 \mathrm{E}-01$ & $\mathrm{Fe}$ \\
1 & 4 & $7.60 \mathrm{E}+00$ & $1.00 \mathrm{E}-01$ & $\mathrm{Fe}$ \\
1 & 5 & $7.60 \mathrm{E}+00$ & $1.00 \mathrm{E}-01$ & $\mathrm{Fe}$ \\
1 & 6 & $7.60 \mathrm{E}+00$ & $1.00 \mathrm{E}-01$ & $\mathrm{Fe}$
\end{tabular}


** RESRAD-BUILD Dose Program Output, Version 3.50 05/03/10 15:05:25 Page: 3 ** Title : Worst Case 1

Input File : sitel.bld

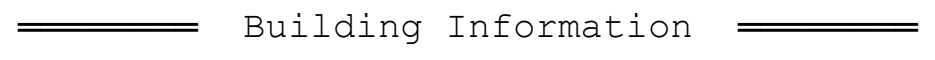

Building Air Exchange Rate: 8.00E-01 1/hr

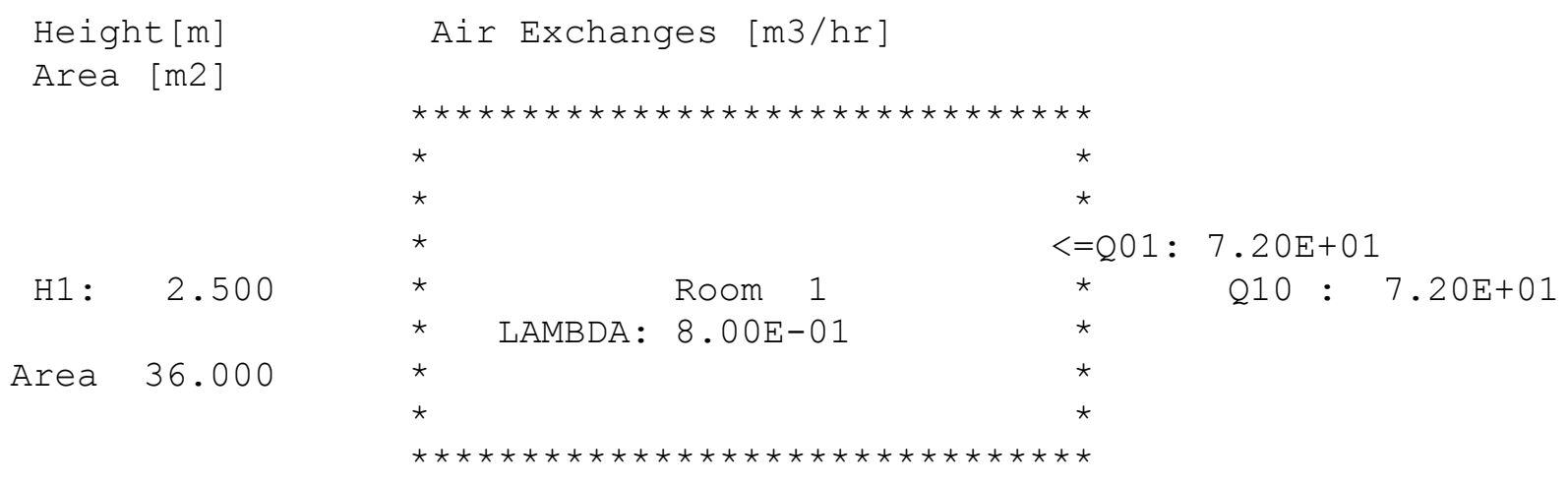

Deposition velocity: 1.00E-02 [m/s] Resuspension Rate: 5.00E-07 [1/s] 
* RESRAD-BUILD Dose Program Output, Version 3.50 05/03/10 15:05:25 Page: 4 ** Title : Worst Case 1

Input File : sitel.bld

$=$ Source Information $=$

Source: 1

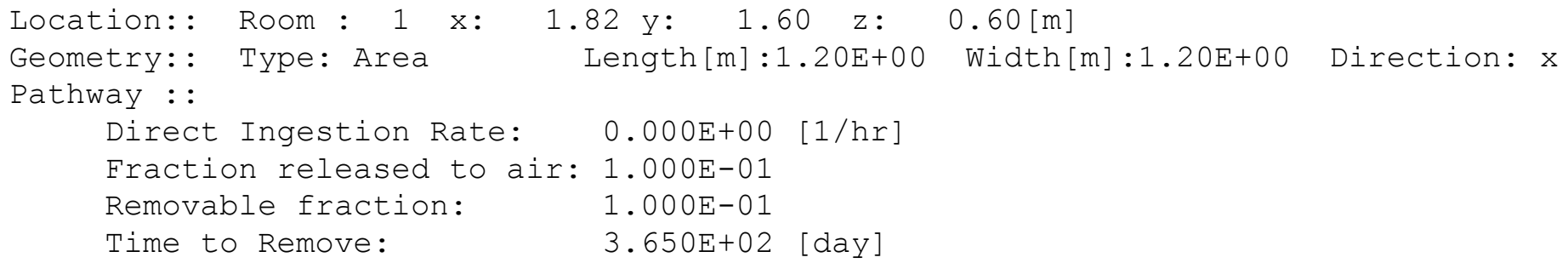

Contamination: :

Nuclide Concentration Dose Conversion Factor (Library: ICRP 72 (Adult))

\begin{tabular}{|c|c|c|c|c|}
\hline & {$[\mathrm{dpm} / \mathrm{m} 2]$} & $\begin{array}{l}\text { Ingestion } \\
\text { [mrem/dpm] }\end{array}$ & $\begin{array}{l}\text { Inhalation } \\
\text { [mrem/dpm] }\end{array}$ & $\begin{array}{l}\text { Submersion } \\
{[\mathrm{mrem} / \mathrm{yr} /} \\
(\mathrm{dpm} / \mathrm{m} 3)]\end{array}$ \\
\hline $\mathrm{U}-235$ & $8.000 \mathrm{E}+03$ & 7.85 & 1.41 & $4.063 \mathrm{E}-04$ \\
\hline$P A-231$ & $0.000 \mathrm{E}+00$ & $1.183 E-03$ & $2.333 E-01$ & $9.049 E-05$ \\
\hline$A C-227$ & $0.000 \mathrm{E}+00$ & $2.015 E-03$ & $9.476 \mathrm{E}-01$ & $9.734 \mathrm{E}-04$ \\
\hline$C S-137$ & $1.500 \mathrm{E}+05$ & $2.167 E-05$ & $6.500 E-05$ & $1.434 E-03$ \\
\hline$S R-90$ & $1.500 \mathrm{E}+05$ & $5.117 E-05$ & $2.692 E-04$ & $1.039 E-05$ \\
\hline
\end{tabular}

Source: 2

Location: : Room : $1 \mathrm{x}: 0.00 \mathrm{y}: 1.60 \mathrm{z}: 0.60[\mathrm{~m}]$ Geometry:: Type: Area Length $[\mathrm{m}]: 1.20 \mathrm{E}+00$ Width $[\mathrm{m}]: 1.20 \mathrm{E}+00$ Direction: $\mathrm{x}$ Pathway : :
Direct Ingestion Rate:
$0.000 \mathrm{E}+00 \quad[1 / \mathrm{hr}]$
Fraction released to air: 1.000E-01
Removable fraction: $\quad 1.000 \mathrm{E}-01$
Time to Remove: $\quad 3.650 \mathrm{E}+02$ [day]

Contamination: :

Nuclide Concentration Dose Conversion Factor (Library: ICRP 72 (Adult))

\begin{tabular}{|c|c|c|c|c|}
\hline & {$[\mathrm{dpm} / \mathrm{m} 2]$} & $\begin{array}{l}\text { Ingestion } \\
\text { [mrem/dpm ] }\end{array}$ & $\begin{array}{l}\text { Inhalation } \\
\text { [mrem/dpm }\end{array}$ & $\begin{array}{l}\text { Submersion } \\
{[\mathrm{mrem} / \mathrm{yr} /} \\
(\mathrm{dpm} / \mathrm{m} 3)]\end{array}$ \\
\hline $\mathrm{U}-235$ & $8.000 E+03$ & $7.890 \mathrm{E}-05$ & $1.417 \mathrm{E}-02$ & $4.063 E-04$ \\
\hline
\end{tabular}


* RESRAD-BUILD Dose Program Output, Version 3.50 05/03/10 15:05:25 Page: 5 ** Title : Worst Case 1

Input File : sitel.bld

$\begin{array}{lllll}\mathrm{PA}-231 & 0.000 \mathrm{E}+00 & 1.183 \mathrm{E}-03 & 2.333 \mathrm{E}-01 & 9.049 \mathrm{E}-05 \\ \mathrm{AC}-227 & 0.000 \mathrm{E}+00 & 2.015 \mathrm{E}-03 & 9.476 \mathrm{E}-01 & 9.734 \mathrm{E}-04 \\ \mathrm{CS}-137 & 1.100 \mathrm{E}+06 & 2.167 \mathrm{E}-05 & 6.500 \mathrm{E}-05 & 1.434 \mathrm{E}-03 \\ \mathrm{SR}-90 & 1.100 \mathrm{E}+06 & 5.117 \mathrm{E}-05 & 2.692 \mathrm{E}-04 & 1.039 \mathrm{E}-05\end{array}$

Source: 3

Location: : Room: 1 x: $0.92 \mathrm{y}: 1.00 \mathrm{z}: 0.60[\mathrm{~m}]$

Geometry:: Type: Area Length $[\mathrm{m}]: 1.83 \mathrm{E}+00$ Width $[\mathrm{m}]: 1.20 \mathrm{E}+00$ Direction: y

Pathway : :

Direct Ingestion Rate: $0.000 \mathrm{E}+00$ [1/hr]

Fraction released to air: 1.000E-01

Removable fraction: $\quad 1.000 \mathrm{E}-01$

Time to Remove: $\quad 3.650 \mathrm{E}+02$ [day]

Contamination: :

Nuclide Concentration Dose Conversion Factor (Library: ICRP 72 (Adult))

\begin{tabular}{|c|c|c|c|c|}
\hline & {$[\mathrm{dpm} / \mathrm{m} 2]$} & $\begin{array}{l}\text { Ingestion } \\
\text { [mrem/dpm ] }\end{array}$ & $\begin{array}{l}\text { Inhalation } \\
\text { [mrem/dpm] }\end{array}$ & $\begin{array}{l}\text { Submersion } \\
{[\mathrm{mrem} / \mathrm{yr} /} \\
(\mathrm{dpm} / \mathrm{m} 3)]\end{array}$ \\
\hline $\mathrm{U}-235$ & $8.000 \mathrm{E}+03$ & $7.890 \mathrm{E}-05$ & $1.417 \mathrm{E}-02$ & $4.063 E-04$ \\
\hline$P A-231$ & $0.000 \mathrm{E}+00$ & $1.183 E-03$ & $2.333 E-01$ & $9.049 E-05$ \\
\hline$A C-227$ & $0.000 \mathrm{E}+00$ & $2.015 E-03$ & $9.476 E-01$ & $9.734 E-04$ \\
\hline$C S-137$ & $1.100 \mathrm{E}+06$ & $2.167 \mathrm{E}-05$ & $6.500 \mathrm{E}-05$ & $1.434 E-03$ \\
\hline$S R-90$ & $1.100 \mathrm{E}+06$ & $5.117 \mathrm{E}-05$ & $2.692 \mathrm{E}-04$ & $1.039 E-05$ \\
\hline
\end{tabular}

Source: 4

Location: Room : $1 \mathrm{x}: 0.92 \mathrm{y}: 2.20 \mathrm{z}: 0.60[\mathrm{~m}]$ Geometry:: Type: Area Length $[\mathrm{m}]: 1.83 \mathrm{E}+00$ Width $[\mathrm{m}]: 1.20 \mathrm{E}+00$ Direction: y Pathway : :
Direct Ingestion Rate:
$0.000 \mathrm{E}+00 \quad[1 / \mathrm{hr}]$
Fraction released to air: 1.000E-01
Removable fraction: $\quad 1.000 \mathrm{E}-01$
Time to Remove: $\quad 3.650 \mathrm{E}+02$ [day]

Contamination: :

Nuclide Concentration Dose Conversion Factor (Library: ICRP 72 (Adult))

\begin{tabular}{|c|c|c|c|c|}
\hline & [dpm/m2 ] & $\begin{array}{l}\text { Ingestion } \\
\text { [mrem/dpm] }\end{array}$ & $\begin{array}{l}\text { Inhalation } \\
\text { [mrem/dpm] }\end{array}$ & $\begin{array}{l}\text { Submersion } \\
{[\mathrm{mrem} / \mathrm{yr} /} \\
(\mathrm{dpm} / \mathrm{m} 3)]\end{array}$ \\
\hline
\end{tabular}


** RESRAD-BUILD Dose Program Output, Version 3.50 05/03/10 15:05:25 Page: $6 * *$ Title : Worst Case 1

Input File : sitel.bld

$\begin{array}{lllll}\mathrm{U}-235 & 8.000 \mathrm{E}+03 & 7.890 \mathrm{E}-05 & 1.417 \mathrm{E}-02 & 4.063 \mathrm{E}-04 \\ \mathrm{PA}-231 & 0.000 \mathrm{E}+00 & 1.183 \mathrm{E}-03 & 2.333 \mathrm{E}-01 & 9.049 \mathrm{E}-05 \\ \mathrm{AC}-227 & 0.000 \mathrm{E}+00 & 2.015 \mathrm{E}-03 & 9.476 \mathrm{E}-01 & 9.734 \mathrm{E}-04 \\ \mathrm{CS}-137 & 1.100 \mathrm{E}+06 & 2.167 \mathrm{E}-05 & 6.500 \mathrm{E}-05 & 1.434 \mathrm{E}-03 \\ \mathrm{SR}-90 & 1.100 \mathrm{E}+06 & 5.117 \mathrm{E}-05 & 2.692 \mathrm{E}-04 & 1.039 \mathrm{E}-05\end{array}$

Source: 5

Location: : Room: $1 \mathrm{x}: 0.92 \mathrm{y}: 1.60 \mathrm{z}: 0.00[\mathrm{~m}]$

Geometry:: Type: Area Length $[\mathrm{m}]: 1.83 \mathrm{E}+00$ Width $[\mathrm{m}]: 1.20 \mathrm{E}+00$ Direction: z

Pathway : :

Direct Ingestion Rate: $0.000 \mathrm{E}+00$ [1/hr]

Fraction released to air: 1.000E-01

Removable fraction: $\quad 1.000 \mathrm{E}-01$

Time to Remove: 3.650E+02 [day]

Contamination: :

Nuclide Concentration Dose Conversion Factor (Library: ICRP 72 (Adult))

\begin{tabular}{|c|c|c|c|c|}
\hline & {$[\mathrm{dpm} / \mathrm{m} 2]$} & $\begin{array}{l}\text { Ingestion } \\
\text { [mrem/dpm] }\end{array}$ & $\begin{array}{l}\text { Inhalation } \\
\text { [mrem/dpm }\end{array}$ & $\begin{array}{l}\text { Submersion } \\
{[\mathrm{mrem} / \mathrm{yr} /} \\
(\mathrm{dpm} / \mathrm{m} 3)]\end{array}$ \\
\hline $\mathrm{U}-23$ & 8.00 & 7.8 & 1.417 & $4.063 E-04$ \\
\hline$P A-231$ & 0.0 & 1.1 & $2.333 E-01$ & $9 E-05$ \\
\hline$A C-227$ & $0.000 \mathrm{E}+00$ & $2.015 E-03$ & $9.476 \mathrm{E}-01$ & $9.734 \mathrm{E}-04$ \\
\hline$C S-137$ & $1.100 \mathrm{E}+06$ & $2.167 E-05$ & $6.500 E-05$ & $1.434 \mathrm{E}-03$ \\
\hline SR-90 & $1.100 \mathrm{E}+06$ & $5.117 \mathrm{E}-05$ & $2.692 \mathrm{E}-04$ & $1.039 E-05$ \\
\hline
\end{tabular}


* RESRAD-BUILD Dose Program Output, Version 3.50 05/03/10 15:05:25 Page: 7 ** Title : Worst Case 1

Input File : sitel.bld

Source: 6

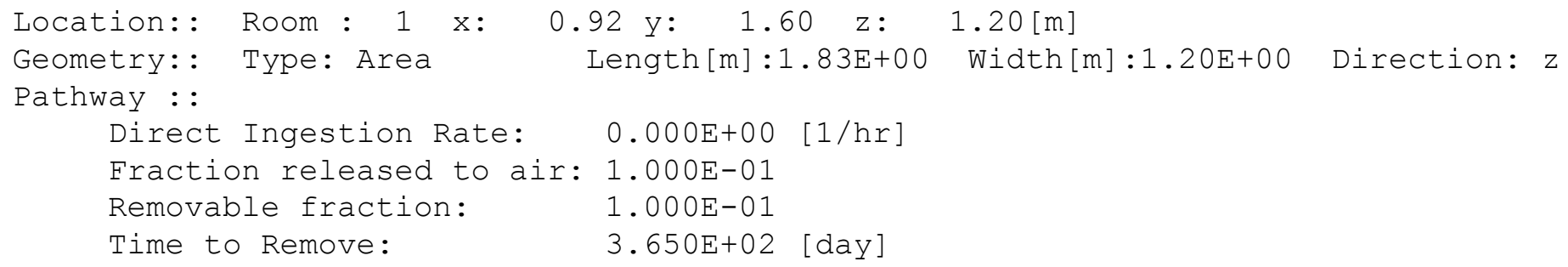

Contamination: :

Nuclide Concentration Dose Conversion Factor (Library: ICRP 72 (Adult))

\begin{tabular}{llllll}
\cline { 5 - 6 } \cline { 5 - 6 } & & & $\begin{array}{c}\text { Ingestion } \\
{[\mathrm{dpm} / \mathrm{m} 2]}\end{array}$ & $\begin{array}{c}\text { Inhalation } \\
{[\mathrm{dpm}]}\end{array}$ & $\begin{array}{c}\text { Submersion } \\
{[\mathrm{mrem} / \mathrm{y} r /}\end{array}$ \\
$\mathrm{U}-235$ & $8.000 \mathrm{E}+03$ & $7.890 \mathrm{E}-05$ & $1.417 \mathrm{E}-02$ & $4.063 \mathrm{E}-04$ \\
$\mathrm{PA}-231$ & $0.000 \mathrm{E}+00$ & $1.183 \mathrm{E}-03$ & $2.333 \mathrm{E}-01$ & $9.049 \mathrm{E}-05$ \\
$\mathrm{AC}-227$ & $0.000 \mathrm{E}+00$ & $2.015 \mathrm{E}-03$ & $9.476 \mathrm{E}-01$ & $9.734 \mathrm{E}-04$ \\
$\mathrm{CS}-137$ & $1.100 \mathrm{E}+06$ & $2.167 \mathrm{E}-05$ & $6.500 \mathrm{E}-05$ & $1.434 \mathrm{E}-03$ \\
$\mathrm{SR}-90$ & $1.100 \mathrm{E}+06$ & $5.117 \mathrm{E}-05$ & $2.692 \mathrm{E}-04$ & $1.039 \mathrm{E}-05$
\end{tabular}


** RESRAD-BUILD Dose Program Output, Version 3.50 05/03/10 15:05:25 Page: 8 ** Title : Worst Case 1

Input File : sitel.bld

Evaluation Time: $0.00000000 \mathrm{E}+00$ years

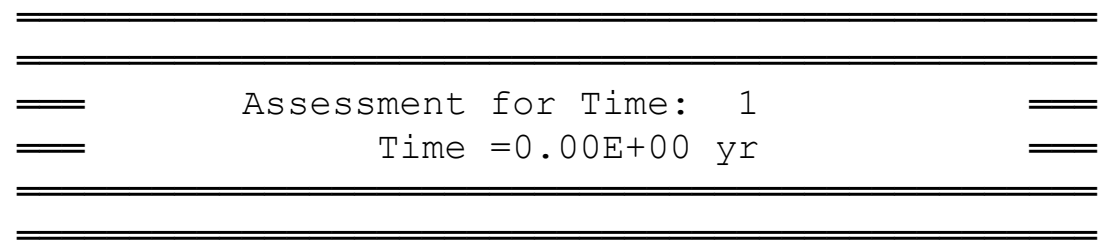

$\overline{=}$ Source Information $=$

Source: 1

Location: : Room : $1 \mathrm{x}: 1.82 \mathrm{y}: 1.60 \mathrm{z}: 0.60$ [m]

Geometry:: Type: Area Length $[\mathrm{m}]: 1.20 \mathrm{E}+00$ Width $[\mathrm{m}]: 1.20 \mathrm{E}+00$ Direction: $\mathrm{x}$

Pathway : :

Direct Ingestion Rate: $0.000 \mathrm{E}+00$ [1/hr]

Fraction released to air: 1.000E-01

Removable fraction: $\quad 1.000 \mathrm{E}-01$

Time to Remove: 3.650E+02 [day]

\begin{tabular}{|c|c|c|}
\hline \multirow[t]{6}{*}{ Contamination: : } & Nuclide & $\begin{array}{c}\text { Concentration } \\
{[\mathrm{dpm} / \mathrm{m} 2]}\end{array}$ \\
\hline & $\mathrm{U}-235$ & $8.000 \mathrm{E}+03$ \\
\hline & $\mathrm{PA}-231$ & $0.000 \mathrm{E}+00$ \\
\hline & $A C-227$ & $0.000 \mathrm{E}+00$ \\
\hline & $C S-137$ & $1.500 E+05$ \\
\hline & $S R-90$ & $1500 F+05$ \\
\hline
\end{tabular}

Source: 2

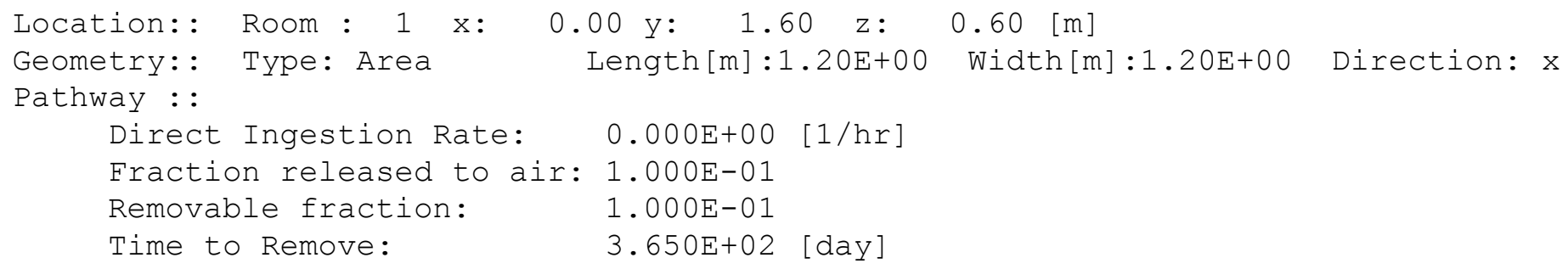

\begin{tabular}{|c|c|c|}
\hline \multirow[t]{6}{*}{ Contamination: : } & Nuclide & $\begin{array}{c}\text { Concentration } \\
{[\mathrm{dom} / \mathrm{m} 2]}\end{array}$ \\
\hline & $\mathrm{U}-235$ & $8.000 E+03$ \\
\hline & $P A-231$ & $0.000 \mathrm{E}+00$ \\
\hline & $A C-227$ & $0.000 \mathrm{E}+00$ \\
\hline & $\mathrm{CS}-137$ & $1.100 \mathrm{E}+06$ \\
\hline & $S R-90$ & $1.100 \mathrm{E}+06$ \\
\hline
\end{tabular}


** RESRAD-BUILD Dose Program Output, Version 3.50 05/03/10 15:05:25 Page: $9 * *$ Title : Worst Case 1

Input File : sitel.bld

Evaluation Time: $0.00000000 \mathrm{E}+00$ years

\section{Source: 3}

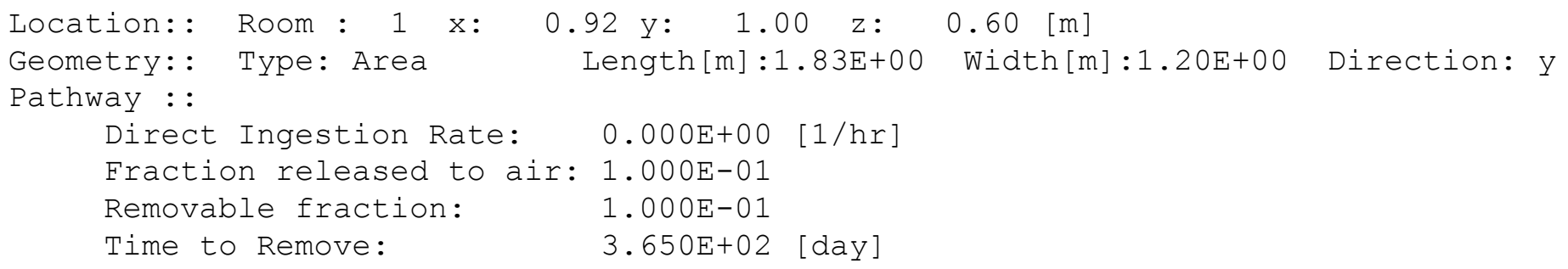

\begin{tabular}{|c|c|c|}
\hline \multirow[t]{6}{*}{ Contamination: : } & Nuclide & $\begin{array}{c}\text { Concentration } \\
{[\mathrm{dpm} / \mathrm{m} 2]}\end{array}$ \\
\hline & $\mathrm{U}-235$ & $8.000 E+03$ \\
\hline & $P A-231$ & $0.000 \mathrm{E}+00$ \\
\hline & $A C-227$ & $0.000 \mathrm{E}+00$ \\
\hline & $\mathrm{CS}-137$ & $1.100 \mathrm{E}+06$ \\
\hline & $S R-90$ & $1.100 E+06$ \\
\hline
\end{tabular}

Source: 4

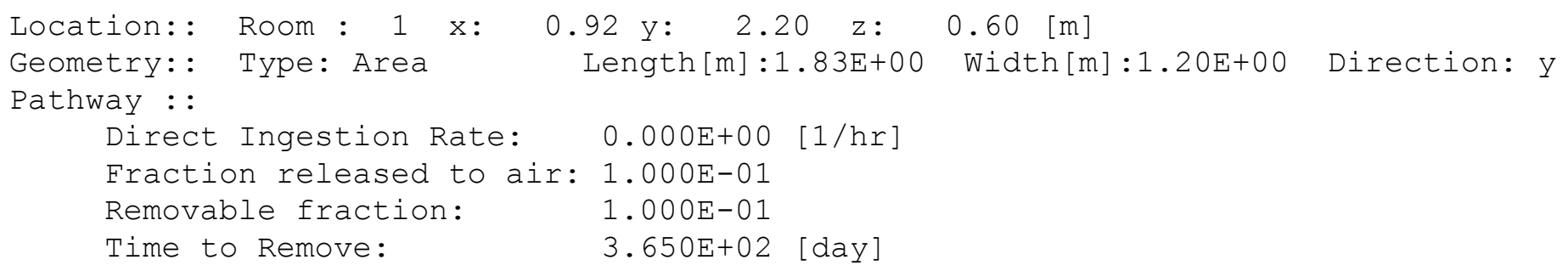


** RESRAD-BUILD Dose Program Output, Version 3.50 05/03/10 15:05:25 Page: 10 ** Title : Worst Case 1

Input File : sitel.bld

Evaluation Time: $0.00000000 \mathrm{E}+00$ years

Source: 5

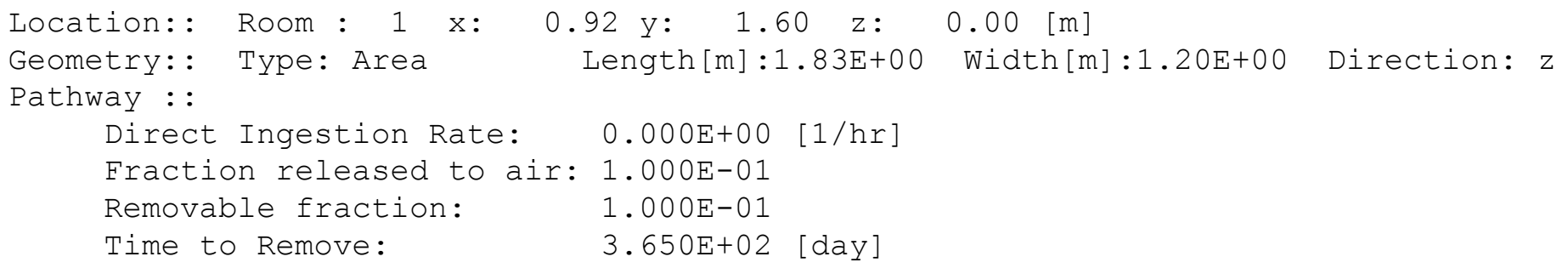

Source: 6

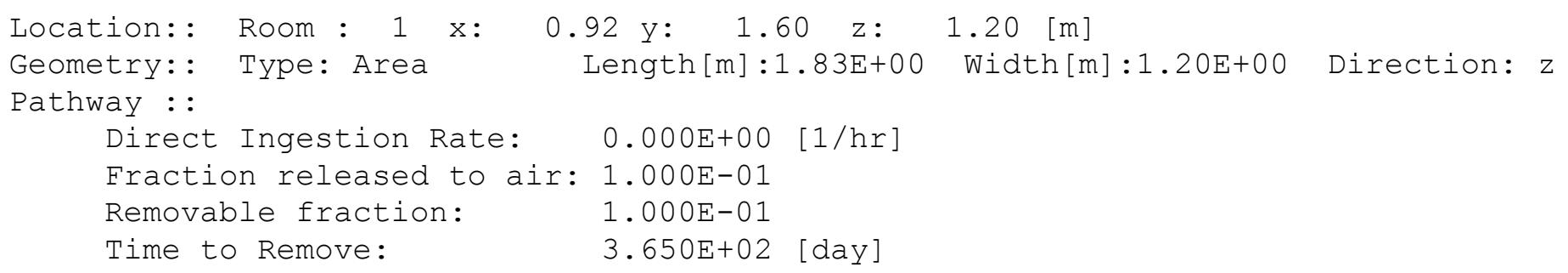


** RESRAD-BUILD Dose Program Output, Version 3.50 05/03/10 15:05:25 Page: 11 ** Title : Worst Case 1

Input File : sitel.bld

Evaluation Time: $0.00000000 \mathrm{E}+00$ years

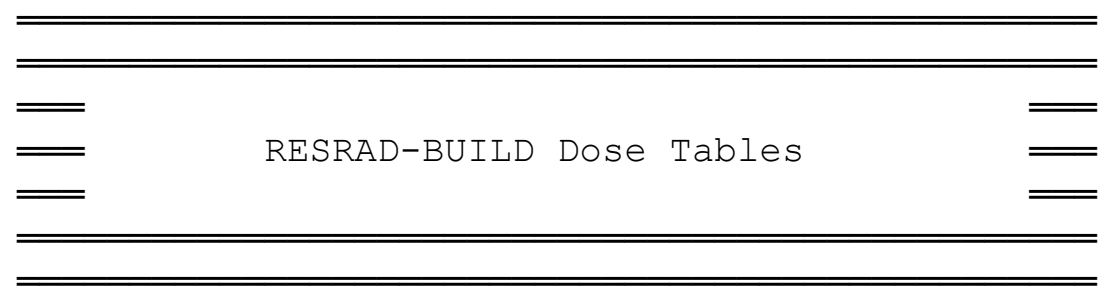

Source Contributions to Receptor Doses

=[mrem]

$[\mathrm{mrem}]$

Source Source source source source source Total

$\begin{array}{lllllll}1 & 2 & 3 & 4 & 5 & 6\end{array}$

Receptor 1 $\begin{array}{llllllll}4.69 \mathrm{E}-02 & 2.94 \mathrm{E}-01 & 3.07 \mathrm{E}-01 & 2.04 \mathrm{E}+00 & 5.74 \mathrm{E}-01 & 5.74 \mathrm{E}-01 & 3.83 \mathrm{E}+00\end{array}$

Total

$\begin{array}{llllllllll}4.69 \mathrm{E}-02 & 2.94 \mathrm{E}-01 & 3.07 \mathrm{E}-01 & 2.04 \mathrm{E}+00 & 5.74 \mathrm{E}-01 & 5.74 \mathrm{E}-01 & 3.83 \mathrm{E}+00\end{array}$ 
** RESRAD-BUILD Dose Program Output, Version 3.50 05/03/10 15:05:25 Page: 12 ** Title : Worst Case 1

Input File : sitel.bld

Evaluation Time: $0.00000000 \mathrm{E}+00$ years

Pathway Detail of Doses

[mrem]

Source: 1

Receptor 1

Total
3. $71 \mathrm{E}-02$

3. $71 \mathrm{E}-02$
Deposition Immersion 3. $79 \mathrm{E}-04$

$3.79 \mathrm{E}-04$
1. 59E-06

1. $59 \mathrm{E}-06$
Inhalation

7. $95 \mathrm{E}-03$

7. $95 \mathrm{E}-03$
Radon $0.00 \mathrm{E}+00$

$0.00 \mathrm{E}+00$
Ingestion

$1.46 \mathrm{E}-03$

1. $46 \mathrm{E}-03$
Source: 2

Receptor 1

Total
External

$2.58 \mathrm{E}-01$

$2.58 \mathrm{E}-01$
Deposition Immersion

2.74E-03

$2.74 \mathrm{E}-03$

Inhalation

Radon 2.30E-02

$2.30 E-02$

$0.00 \mathrm{E}+00$

Ingestion

1. $02 \mathrm{E}-02$

$1.02 \mathrm{E}-02$

Source: 3

Receptor 1

Total

Source: 4

Receptor 1

Total

Source: 5

Receptor 1

Total

Source: 6

Receptor 1

Total
External

2. $52 \mathrm{E}-01$

$2.52 \mathrm{E}-01$
Deposition Immersion

4.17E-03

1. $75 \mathrm{E}-05$

$4.17 \mathrm{E}-03$

1. $75 \mathrm{E}-05$
Inhalation Radon 3. $51 \mathrm{E}-02$

$3.51 \mathrm{E}-02$

$0.00 \mathrm{E}+00$

$0.00 \mathrm{E}+00$
Ingestion

1. 55E-02

1. 55E-02
External

1. $98 \mathrm{E}+00$

1. $98 \mathrm{E}+00$ $\begin{array}{ll}\text { Deposition } & \text { Immersion } \\ 4.17 \mathrm{E}-03 & 1.75 \mathrm{E}-05 \\ 4.17 \mathrm{E}-03 & 1.75 \mathrm{E}-05\end{array}$
Inhalation

3. 51E-02

$3.51 \mathrm{E}-02$
Radon

$0.00 \mathrm{E}+00$

$0.00 \mathrm{E}+00$
Ingestion

1. 55E-02

1. 55E-02
External

5.20E-01

5. $20 \mathrm{E}-01$ $\begin{array}{ll}\text { Deposition } & \text { Immersion } \\ 4.17 \mathrm{E}-03 & 1.75 \mathrm{E}-05 \\ 4.17 \mathrm{E}-03 & 1.75 \mathrm{E}-05\end{array}$
Inhalation

3. 51E-02

$3.51 \mathrm{E}-02$
Radon

$0.00 \mathrm{E}+00$

$0.00 \mathrm{E}+00$
Ingestion

1. 55E-02

1. $55 \mathrm{E}-02$
External

5.20E-01

5. 20E-01 $\begin{array}{ll}\text { Deposition } & \text { Immersion } \\ 4.17 \mathrm{E}-03 & 1.75 \mathrm{E}-05 \\ 4.17 \mathrm{E}-03 & 1.75 \mathrm{E}-05\end{array}$

Inhalation Radon

3.51E-02 0.00E+00

3. $51 \mathrm{E}-02 \quad 0.00 \mathrm{E}+00$
Ingestion

1. 55E-02

1. $55 \mathrm{E}-02$ 
** RESRAD-BUILD Dose Program Output, Version 3.50 05/03/10 15:05:25 Page: 13 ** Title : Worst Case 1

Input File : sitel.bld

Evaluation Time: $0.00000000 \mathrm{E}+00$ years

Nuclide Detail of Doses

[mrem ]

Source: 1

$\begin{array}{lcc}\text { Nuclide } & \begin{array}{c}\text { Receptor } \\ 1\end{array} & \text { Total } \\ & & \\ \mathrm{U}-235 & 6.29 \mathrm{E}-03 & 6.29 \mathrm{E}-03 \\ \mathrm{PA}-231 & 9.64 \mathrm{E}-07 & 9.64 \mathrm{E}-07 \\ \mathrm{AC}-227 & 3.86 \mathrm{E}-08 & 3.86 \mathrm{E}-08 \\ \mathrm{CS}-137 & 3.73 \mathrm{E}-02 & 3.73 \mathrm{E}-02 \\ \text { SR-90 } & 3.25 \mathrm{E}-03 & 3.25 \mathrm{E}-03\end{array}$

Source: 2

\begin{tabular}{|c|c|c|}
\hline Nuclide & $\begin{array}{c}\text { Receptor } \\
1\end{array}$ & Total \\
\hline $\mathrm{U}-235$ & $6.15 \mathrm{E}-03$ & $6.15 \mathrm{E}-03$ \\
\hline$P A-231$ & $9.63 \mathrm{E}-07$ & $9.63 \mathrm{E}-07$ \\
\hline$A C-2$ & $3.86 \mathrm{E}-08$ & $3.86 \mathrm{E}-08$ \\
\hline CS -137 & $2.64 \mathrm{E}-01$ & $2.64 \mathrm{E}-01$ \\
\hline$S R-90$ & $2.34 \mathrm{E}-02$ & $2.34 \mathrm{E}-02$ \\
\hline
\end{tabular}

Source: 3

Nuclide Receptor Total

1

$\begin{array}{lll}\mathrm{U}-235 & 9.12 \mathrm{E}-03 & 9.12 \mathrm{E}-03\end{array}$

$\mathrm{PA}-231 \quad 1.47 \mathrm{E}-06 \quad 1.47 \mathrm{E}-06$

$\begin{array}{lll}\mathrm{AC}-227 & 5.88 \mathrm{E}-08 \quad 5.88 \mathrm{E}-08\end{array}$

$\begin{array}{lll}C S-137 & 2.63 \mathrm{E}-01 & 2.63 \mathrm{E}-01\end{array}$

SR-90 3.44E-02 3.44E-02 
** RESRAD-BUILD Dose Program Output, Version 3.50 05/03/10 15:05:25 Page: 14 ** Title : Worst Case 1

Input File : sitel.bld

Evaluation Time: $0.00000000 \mathrm{E}+00$ years

\section{Source: 4}

$\begin{array}{lcc}\text { Nuclide } & \begin{array}{c}\text { Receptor } \\ 1\end{array} & \text { Total } \\ & \begin{array}{l}1.23 \mathrm{E}-02 \\ \mathrm{U}-235\end{array} & 1.23 \mathrm{E}-02 \\ \mathrm{PA}-231 & 1.48 \mathrm{E}-06 & 1.48 \mathrm{E}-06 \\ \mathrm{AC}-227 & 5.97 \mathrm{E}-08 & 5.97 \mathrm{E}-08 \\ \mathrm{CS}-137 & 1.97 \mathrm{E}+00 & 1.97 \mathrm{E}+00 \\ \mathrm{SR}-90 & 4.94 \mathrm{E}-02 & 4.94 \mathrm{E}-02\end{array}$

Source: 5

\begin{tabular}{|c|c|c|}
\hline uclide & $\begin{array}{c}\text { Receptor } \\
1\end{array}$ & Total \\
\hline$U-2$ & $9.60 E-03$ & 3 \\
\hline & 1.4 & 1 \\
\hline 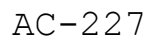 & $5.89 \mathrm{E}-08$ & $\mathrm{~g}-08$ \\
\hline$C S-13$ & $5.28 \mathrm{E}-01$ & $5.28 \mathrm{E}-01$ \\
\hline$S R-90$ & $3.67 \mathrm{E}-02$ & $3.67 \mathrm{E}-02$ \\
\hline
\end{tabular}

Source: 6

Nuclide Receptor Total

1

$\mathrm{U}-2359.60 \mathrm{E}-03 \quad 9.60 \mathrm{E}-03$

PA-231 $1.47 \mathrm{E}-06 \quad 1.47 \mathrm{E}-06$

$\mathrm{AC}-227 \quad 5.89 \mathrm{E}-08 \quad 5.89 \mathrm{E}-08$

$\begin{array}{lll}\mathrm{CS}-137 & 5.28 \mathrm{E}-01 & 5.28 \mathrm{E}-01\end{array}$

SR-90 $3.67 \mathrm{E}-02 \quad 3.67 \mathrm{E}-02$ 
** RESRAD-BUILD Dose Program Output, Version 3.50 05/03/10 15:05:25 Page: 15 ** Title : Worst Case 1

Input File : sitel.bld

Evaluation Time: 1.00000000 years

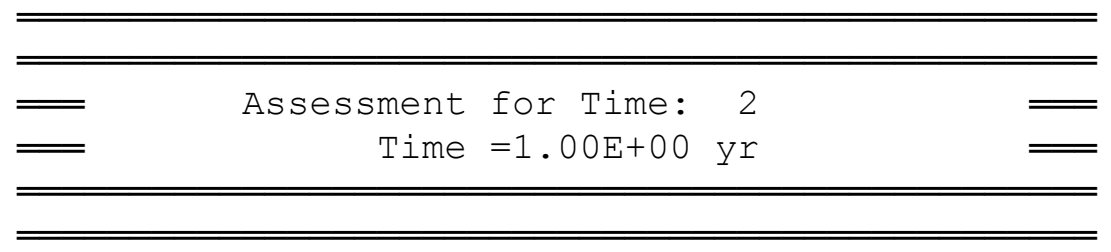

$=$ Source Information $=$

Source: 1

Location: : Room : $1 \mathrm{x}: 1.82 \mathrm{y}: 1.60 \mathrm{z}: 0.60$ [m]

Geometry:: Type: Area Length $[\mathrm{m}]: 1.20 \mathrm{E}+00$ Width $[\mathrm{m}]: 1.20 \mathrm{E}+00$ Direction: $\mathrm{x}$

Pathway : :

Direct Ingestion Rate: $0.000 \mathrm{E}+00$ [1/hr]

Fraction released to air: 1.000E-01

Removable fraction: $\quad 0.000 \mathrm{E}+00$

Time to Remove: $\quad 3.650 \mathrm{E}+02$ [day]

\begin{tabular}{|c|c|c|}
\hline \multirow[t]{6}{*}{ Contamination: : } & Nuclide & $\begin{array}{c}\text { Concentration } \\
{[\mathrm{dpm} / \mathrm{m} 2]}\end{array}$ \\
\hline & $\mathrm{U}-235$ & $7.200 E+03$ \\
\hline & $\mathrm{PA}-231$ & $1.522 \mathrm{E}-01$ \\
\hline & $A C-227$ & $2.393 E-03$ \\
\hline & $C S-137$ & $1.319 E+05$ \\
\hline & $S R-90$ & $1.318 E+05$ \\
\hline
\end{tabular}

Source: 2

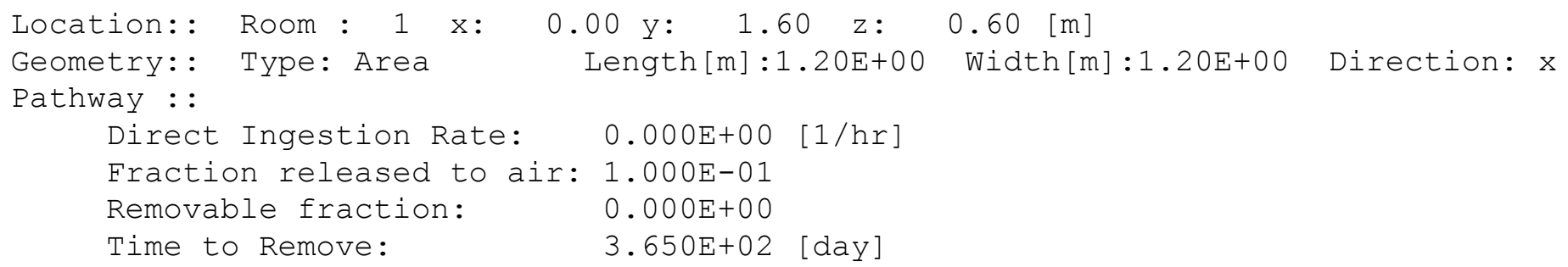


* RESRAD-BUILD Dose Program Output, Version 3.50 05/03/10 15:05:25 Page: 16 ** Title : Worst Case 1

Input File : sitel.bld

Evaluation Time: 1.00000000 years

\section{Source: 3}

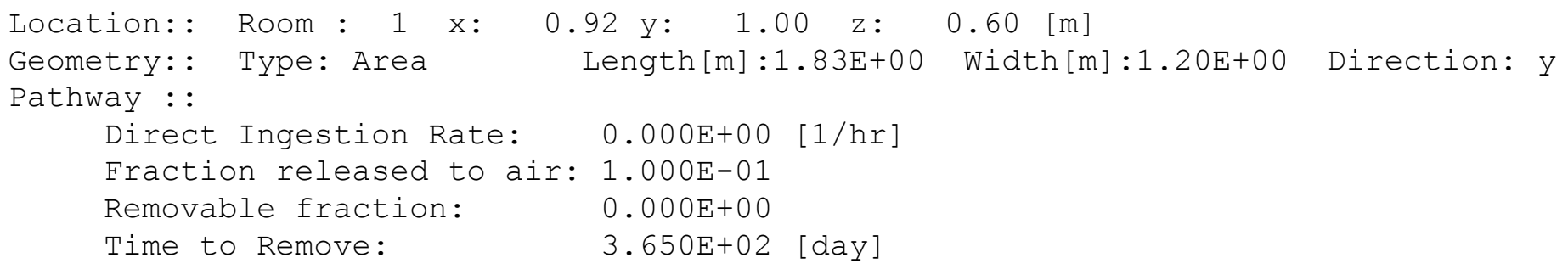

\begin{tabular}{|c|c|c|}
\hline \multirow[t]{6}{*}{ Contamination: : } & Nuclide & $\begin{array}{c}\text { Concentration } \\
{[\mathrm{dpm} / \mathrm{m} 2]}\end{array}$ \\
\hline & $\mathrm{U}-235$ & $7.200 E+03$ \\
\hline & $\mathrm{PA}-231$ & $1.522 \mathrm{E}-01$ \\
\hline & $A C-227$ & $2.393 E-03$ \\
\hline & $\mathrm{CS}-137$ & $9.674 \mathrm{E}+05$ \\
\hline & $S R-90$ & $9.667 E+05$ \\
\hline
\end{tabular}

Source: 4

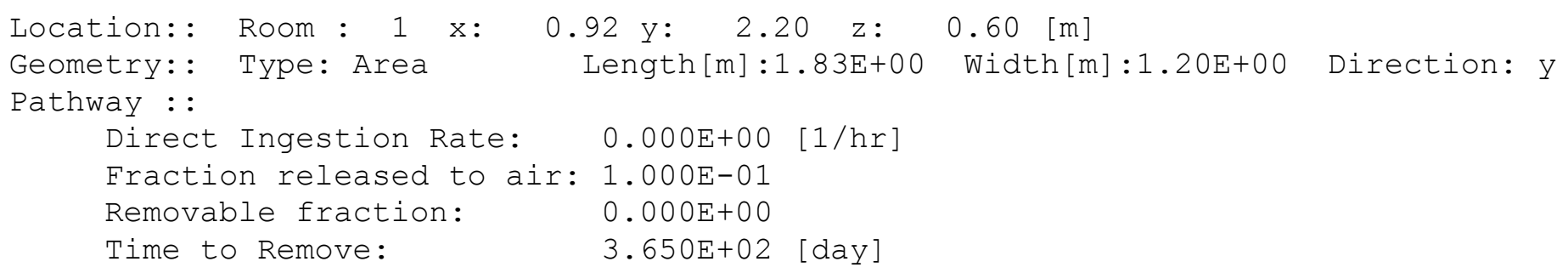


** RESRAD-BUILD Dose Program Output, Version 3.50 05/03/10 15:05:25 Page: 17 ** Title : Worst Case 1

Input File : sitel.bld

Evaluation Time: 1.00000000 years

Source: 5

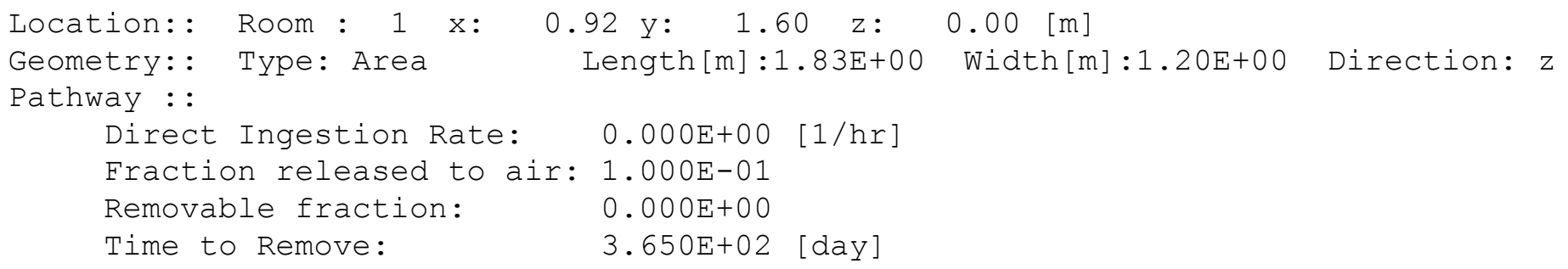

\begin{tabular}{|c|c|c|}
\hline \multirow[t]{6}{*}{ Contamination: : } & Nuclide & $\begin{array}{c}\text { Concentration } \\
{[\mathrm{dpm} / \mathrm{m} 2]}\end{array}$ \\
\hline & $\mathrm{U}-235$ & $7.200 \mathrm{E}+03$ \\
\hline & $\mathrm{PA}-231$ & $1.522 \mathrm{E}-01$ \\
\hline & $A C-227$ & $2.393 E-03$ \\
\hline & $\mathrm{CS}-137$ & $9.674 \mathrm{E}+05$ \\
\hline & $S R-90$ & $9.667 E+05$ \\
\hline
\end{tabular}

Source: 6

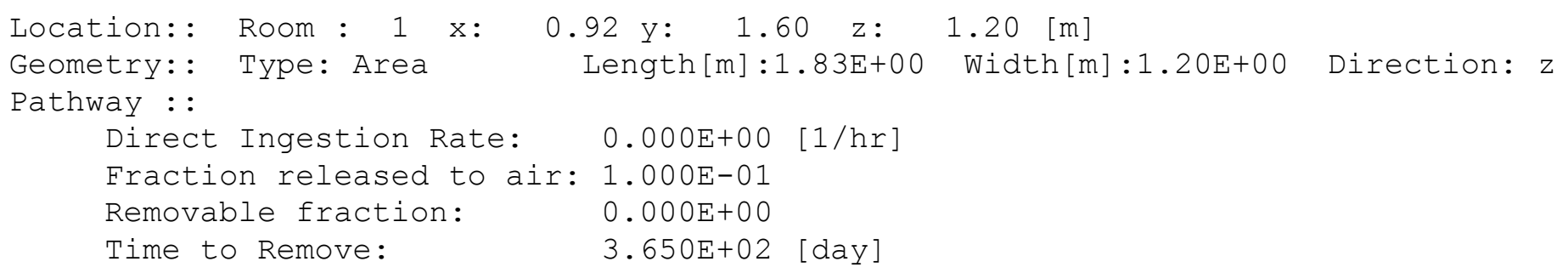


** RESRAD-BUILD Dose Program Output, Version 3.50 05/03/10 15:05:25 Page: 18 ** Title : Worst Case 1

Input File : sitel.bld

Evaluation Time: 1.00000000 years

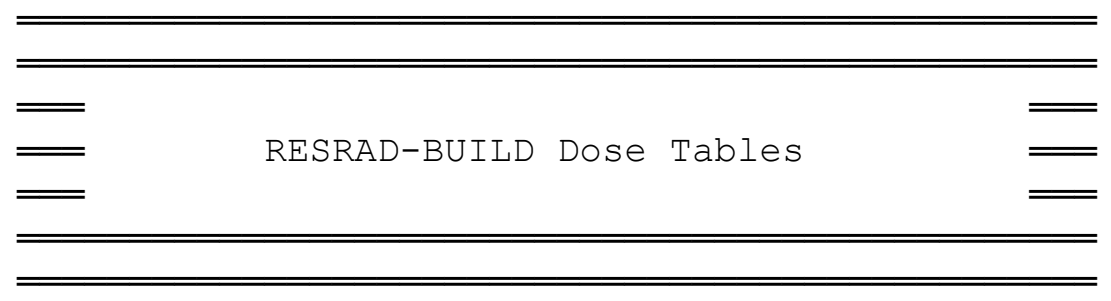

Source Contributions to Receptor Doses

[mrem]

Source Source Source source Source source Total

$\begin{array}{lllllll}1 & 2 & 3 & 4 & 5\end{array}$

Receptor 13 3.43E-02 2.38E-01 2.33E-01 $1.83 \mathrm{E}+00 \quad 4.81 \mathrm{E}-01 \quad 4.81 \mathrm{E}-01 \quad 3.30 \mathrm{E}+00$

$\begin{array}{lllllll}3.43 \mathrm{E}-02 & 2.38 \mathrm{E}-01 & 2.33 \mathrm{E}-01 & 1.83 \mathrm{E}+00 & 4.81 \mathrm{E}-01 & 4.81 \mathrm{E}-01 & 3.30 \mathrm{E}+00\end{array}$ 
** RESRAD-BUILD Dose Program Output, Version 3.50 05/03/10 15:05:25 Page: 19 ** Title : Worst Case 1

Input File : sitel.bld

Evaluation Time: 1.00000000 years

Pathway Detail of Doses

[mrem]

Source: 1

Receptor External

1

Total

3. $43 \mathrm{E}-02$

3. $43 E-02$

External

$2.38 \mathrm{E}-01$

$2.38 \mathrm{E}-01$

Total
Deposition Immersion

$0.00 \mathrm{E}+00$

$0.00 \mathrm{E}+00$

$0.00 \mathrm{E}+00$

$0.00 \mathrm{E}+00$

Inhalation

$0.00 \mathrm{E}+00$

$0.00 \mathrm{E}+00$

Radon

$0.00 \mathrm{E}+00$

$0.00 \mathrm{E}+00$

Ingestion

$0.00 \mathrm{E}+00$

$0.00 \mathrm{E}+00$
Source: 3

Receptor 1

Total

Source: 4

Receptor 1

Total

Source: 5

Receptor 1

Total

Source: 6

Receptor 1

Total
External

2.33E-01

2. $33 \mathrm{E}-01$

External

$1.83 \mathrm{E}+00$

$1.83 \mathrm{E}+00$
Deposition Immersion

$0.00 \mathrm{E}+00$

$0.00 \mathrm{E}+00$

$0.00 \mathrm{E}+00$

$0.00 \mathrm{E}+00$

Inhalation

$0.00 \mathrm{E}+00$

$0.00 \mathrm{E}+00$

Radon

$0.00 \mathrm{E}+00$

$0.00 \mathrm{E}+00$

Ingestion

$0.00 \mathrm{E}+00$

$0.00 \mathrm{E}+00$
Deposition Immersion
Inhalation Radon
Ingestion
$0.00 \mathrm{E}+00$
$0.00 \mathrm{E}+00$
$0.00 \mathrm{E}+00$
$0.00 \mathrm{E}+00$
$0.00 \mathrm{E}+00$
$0.00 \mathrm{E}+00$
$0.00 \mathrm{E}+00$
$0.00 \mathrm{E}+00$
$0.00 \mathrm{E}+00$
$0.00 \mathrm{E}+00$

External

4.81E-01

4. 81E-01 $\begin{array}{ll}\text { Deposition } & \text { Immersion } \\ 0.00 \mathrm{E}+00 & 0.00 \mathrm{E}+00 \\ 0.00 \mathrm{E}+00 & 0.00 \mathrm{E}+00\end{array}$

Inhalation

$0.00 \mathrm{E}+00$

$0.00 \mathrm{E}+00$

Radon

$0.00 \mathrm{E}+00$

$0.00 \mathrm{E}+00$
Ingestion

$0.00 \mathrm{E}+00$

$0.00 \mathrm{E}+00$

External

Deposition Immersion

Inhalation Radon

$0.00 \mathrm{E}+00$

$0.00 \mathrm{E}+00$

$0.00 \mathrm{E}+00$

$0.00 \mathrm{E}+00$

Ingestion

4. $81 \mathrm{E}-01$

$0.00 \mathrm{E}+00$

$0.00 \mathrm{E}+00$

$0.00 \mathrm{E}+00$

$0.00 \mathrm{E}+00$

$00 \mathrm{E}+00$

$0.00 \mathrm{E}+00$ 
** RESRAD-BUILD Dose Program Output, Version 3.50 05/03/10 15:05:25 Page: 20 ** Title : Worst Case 1

Input File : sitel.bld

Evaluation Time: 1.00000000 years

Nuclide Detail of Doses

[mrem ]

Source: 1

\begin{tabular}{|c|c|c|}
\hline Nuclide & $\begin{array}{c}\text { Receptor } \\
1\end{array}$ & Total \\
\hline-235 & $5.87 \mathrm{E}-04$ & $E-04$ \\
\hline $\mathrm{PA}-231$ & $5.28 E-09$ & $5.28 E-09$ \\
\hline$A C-227$ & $1.00 \mathrm{E}-09$ & $1.00 E-09$ \\
\hline CS -137 & $3.34 \mathrm{E}-02$ & $3.34 \mathrm{E}-02$ \\
\hline$S R-90$ & $3.39 \mathrm{E}-04$ & $3.39 \mathrm{E}-04$ \\
\hline
\end{tabular}

Source: 2

$\begin{array}{lcc}\text { Nuclide } & \begin{array}{c}\text { Receptor } \\ 1\end{array} & \text { Total } \\ \text { U-235 } & 4.59 \mathrm{E}-04 & 4.59 \mathrm{E}-04 \\ \mathrm{PA}-231 & 3.43 \mathrm{E}-09 & 3.43 \mathrm{E}-09 \\ \text { AC-227 } & 8.88 \mathrm{E}-10 & 8.88 \mathrm{E}-10 \\ \text { CS-137 } & 2.36 \mathrm{E}-01 & 2.36 \mathrm{E}-01 \\ \text { SR-90 } & 2.07 \mathrm{E}-03 & 2.07 \mathrm{E}-03\end{array}$

Source: 3

\begin{tabular}{|c|c|c|}
\hline Nuclide & Receptor $_{1}$ & Total \\
\hline-2 & $4.54 \mathrm{E}-04$ & $4.54 \mathrm{E}-04$ \\
\hline 1 & $3.37 \mathrm{E}-09$ & $3.37 \mathrm{E}-09$ \\
\hline$C-227$ & $8.79 \mathrm{E}-10$ & $8.79 \mathrm{E}-10$ \\
\hline-137 & $2.31 \mathrm{E}-01$ & $2.31 \mathrm{E}-01$ \\
\hline-90 & $2.02 \mathrm{E}-03$ & $2.02 \mathrm{E}-03$ \\
\hline
\end{tabular}


** RESRAD-BUILD Dose Program Output, Version 3.50 05/03/10 15:05:25 Page: 21 ** Title : Worst Case 1

Input File : sitel.bld

Evaluation Time: 1.00000000 years

\section{Source: 4}

\begin{tabular}{|c|c|c|}
\hline Nuclide & $\begin{array}{c}\text { Receptor } \\
1\end{array}$ & Total \\
\hline $\mathrm{U}-2$ & $3.47 E-03$ & \\
\hline & $2.63 E-08$ & 2 . \\
\hline 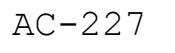 & -09 & $E-09$ \\
\hline CS-137 & $1.81 \mathrm{E}+00$ & $1.81 \mathrm{E}+00$ \\
\hline$R-90$ & $1.59 \mathrm{E}-02$ & $1.59 \mathrm{E}-02$ \\
\hline
\end{tabular}

Source: 5

$\begin{array}{lcc}\text { Nuclide } & \text { Receptor } & \text { Total } \\ & 1 & \\ \mathrm{U}-235 & 9.09 \mathrm{E}-04 & 9.09 \mathrm{E}-04 \\ \mathrm{PA}-231 & 6.90 \mathrm{E}-09 & 6.90 \mathrm{E}-09 \\ \mathrm{AC}-227 & 1.76 \mathrm{E}-09 & 1.76 \mathrm{E}-09 \\ \mathrm{CS}-137 & 4.76 \mathrm{E}-01 & 4.76 \mathrm{E}-01 \\ \text { SR-90 } & 4.16 \mathrm{E}-03 & 4.16 \mathrm{E}-03\end{array}$

Source: 6

Nuclide Receptor Total

1

$\mathrm{U}-2359.09 \mathrm{E}-04 \quad 9.09 \mathrm{E}-04$

$\begin{array}{lll}\mathrm{PA}-231 & 6.90 \mathrm{E}-09 & 6.90 \mathrm{E}-09\end{array}$

$\mathrm{AC}-227 \quad 1.76 \mathrm{E}-09 \quad 1.76 \mathrm{E}-09$

CS-137 4.76E-01 4.76E-01

SR-90 4.16E-03 4.16E-03 
* RESRAD-BUILD Dose Program Output, Version 3.50 05/03/10 15:05:25 Page: 22 ** Title : Worst Case 1

Input File : sitel.bld

Evaluation Time: 10.0000000 years

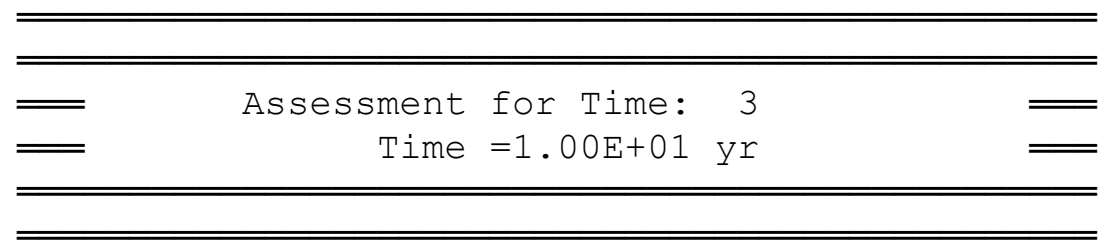

$\overline{=}$ Source Information $=$

Source: 1

Location: : Room : $1 \mathrm{x}: 1.82 \mathrm{y}: 1.60 \mathrm{z}: 0.60$ [m]

Geometry:: Type: Area Length [m]:1.20E+00 Width[m]:1.20E+00 Direction: $x$

Pathway : :

Direct Ingestion Rate: $0.000 \mathrm{E}+00$ [1/hr]

Fraction released to air: 1.000E-01

Removable fraction: $\quad 0.000 \mathrm{E}+00$

Time to Remove: $\quad 3.650 \mathrm{E}+02$ [day]

\begin{tabular}{|c|c|c|}
\hline \multirow[t]{6}{*}{ Contamination: : } & Nuclide & $\begin{array}{c}\text { Concentration } \\
{[\mathrm{dpm} / \mathrm{m} 2]}\end{array}$ \\
\hline & $\mathrm{U}-235$ & $7.200 \mathrm{E}+03$ \\
\hline & $\mathrm{PA}-231$ & $1.521 \mathrm{E}+00$ \\
\hline & $A C-227$ & $2.182 \mathrm{E}-01$ \\
\hline & $\mathrm{CS}-137$ & $1.071 E+05$ \\
\hline & $S R-90$ & $1.064 E+05$ \\
\hline
\end{tabular}

Source: 2

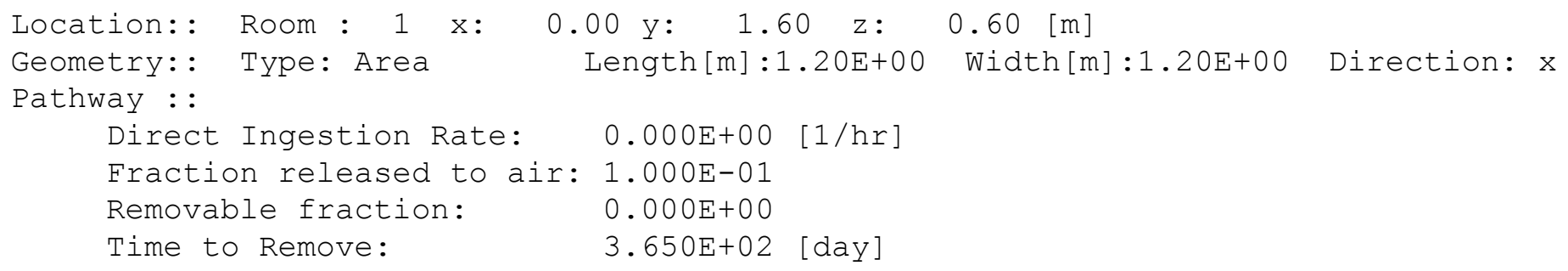


** RESRAD-BUILD Dose Program Output, Version 3.50 05/03/10 15:05:25 Page: 23 ** Title : Worst Case 1

Input File : sitel.bld

Evaluation Time: 10.0000000 years

\section{Source: 3}

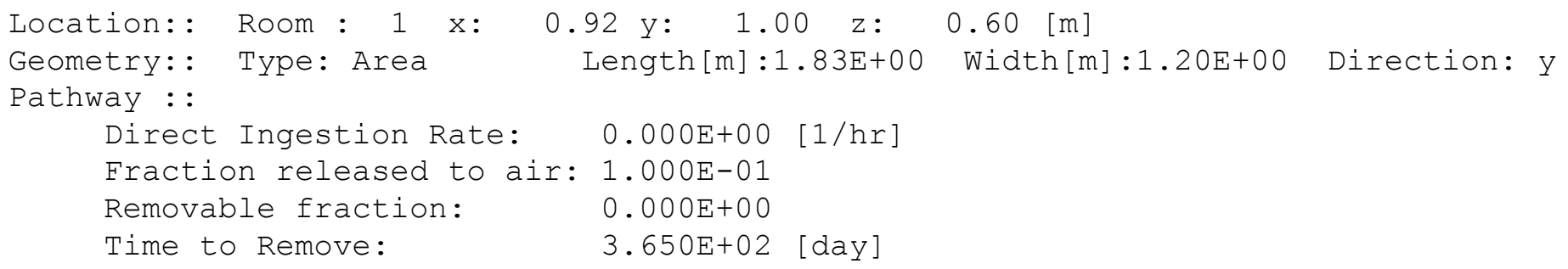

\begin{tabular}{|c|c|c|}
\hline \multirow[t]{6}{*}{ Contamination: : } & Nuclide & $\begin{array}{c}\text { Concentration } \\
{[\mathrm{dpm} / \mathrm{m} 2]}\end{array}$ \\
\hline & $\mathrm{U}-235$ & $7.200 E+03$ \\
\hline & $\mathrm{PA}-231$ & $1.521 \mathrm{E}+00$ \\
\hline & $A C-227$ & $2.182 \mathrm{E}-01$ \\
\hline & $C S-137$ & $7.858 E+05$ \\
\hline & $S R-90$ & $7.802 E+05$ \\
\hline
\end{tabular}

Source: 4

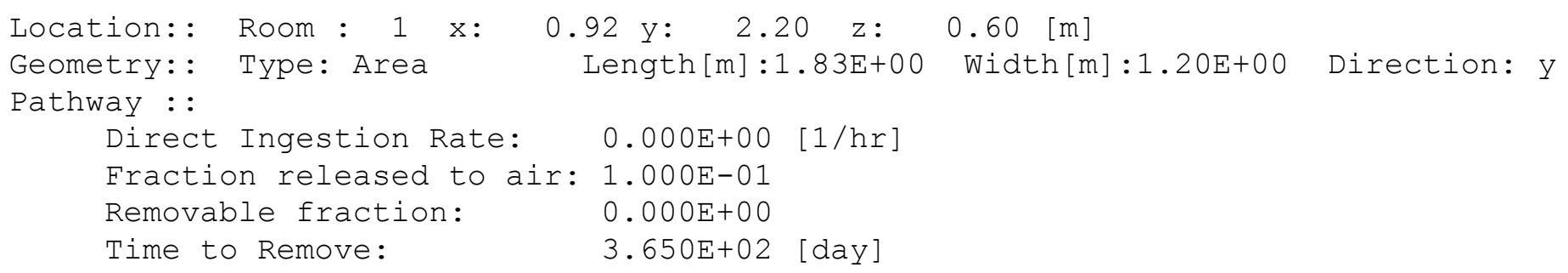


** RESRAD-BUILD Dose Program Output, Version 3.50 05/03/10 15:05:25 Page: 24 ** Title : Worst Case 1

Input File : sitel.bld

Evaluation Time: 10.0000000 years

Source: 5

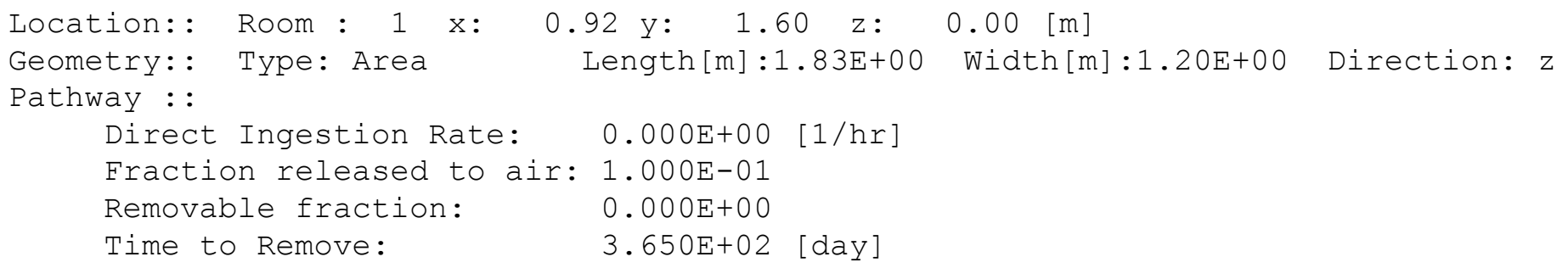

\begin{tabular}{|c|c|c|}
\hline \multirow[t]{6}{*}{ Contamination: : } & Nuclide & $\begin{array}{c}\text { Concentration } \\
{[\mathrm{dpm} / \mathrm{m} 2]}\end{array}$ \\
\hline & $\mathrm{U}-235$ & $7.200 E+03$ \\
\hline & $P A-231$ & $1.521 E+00$ \\
\hline & $A C-227$ & $2.182 \mathrm{E}-01$ \\
\hline & $C S-137$ & $7.858 E+05$ \\
\hline & $S R-90$ & $7.802 E+05$ \\
\hline
\end{tabular}

Source: 6

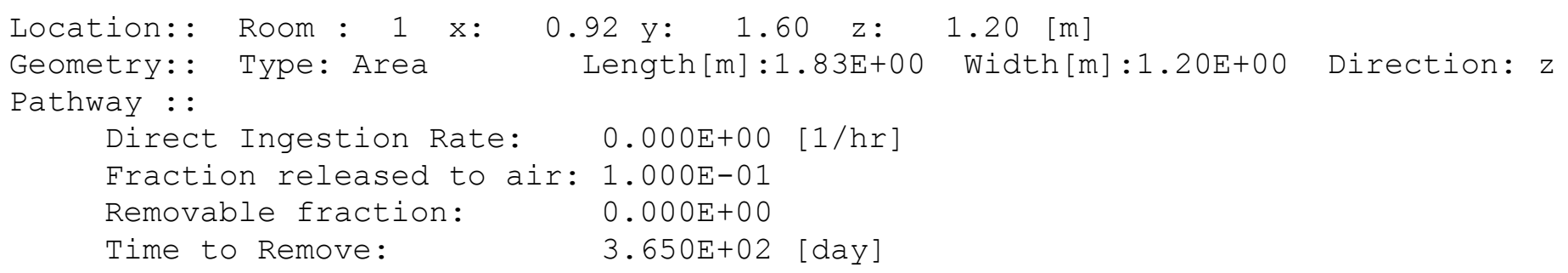


** RESRAD-BUILD Dose Program Output, Version 3.50 05/03/10 15:05:25 Page: 25 ** Title : Worst Case 1

Input File : sitel.bld

Evaluation Time: 10.0000000 years

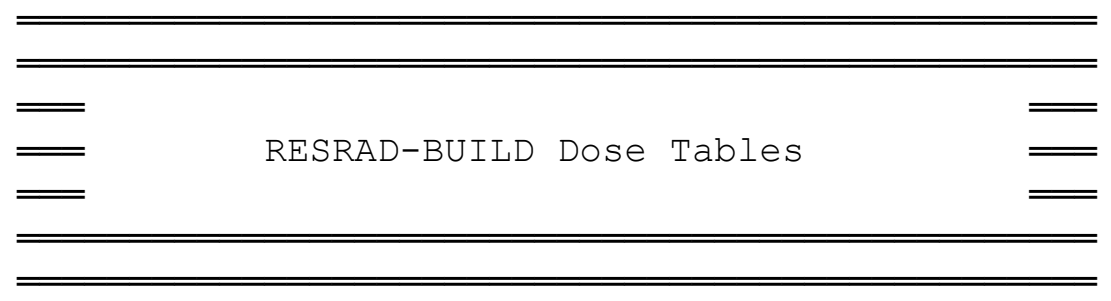

Source Contributions to Receptor Doses

=

$[\mathrm{mrem}]$

Source Source Source Source source source Total

$\begin{array}{lllllll}1 & 2 & 3 & 4 & 5\end{array}$

$\begin{array}{llllllllllllll}\text { Receptor } 1 & 2.80 \mathrm{E}-02 & 1.94 \mathrm{E}-01 & 1.90 \mathrm{E}-01 & 1.49 \mathrm{E}+00 & 3.91 \mathrm{E}-01 & 3.91 \mathrm{E}-01 & 2.68 \mathrm{E}+00\end{array}$

Total

$\begin{array}{lllllllll}2.80 \mathrm{E}-02 & 1.94 \mathrm{E}-01 & 1.90 \mathrm{E}-01 & 1.49 \mathrm{E}+00 & 3.91 \mathrm{E}-01 & 3.91 \mathrm{E}-01 & 2.68 \mathrm{E}+00\end{array}$ 
** RESRAD-BUILD Dose Program Output, Version 3.50 05/03/10 15:05:25 Page: $26 * \star$ Title : Worst Case 1

Input File : sitel.bld

Evaluation Time: 10.0000000 years

Pathway Detail of Doses

[mrem ]

Source: 1

Receptor External

1

Total

$2.80 \mathrm{E}-02$

2. $80 \mathrm{E}-02$

External

1.94E-01

1. $94 \mathrm{E}-01$

Total ptor

1
Source: 2

$\begin{array}{cl}\text { Receptor } & \text { External } \\ 1 & 1.94 \mathrm{E}-01 \\ \text { Total } & 1.94 \mathrm{E}-01\end{array}$

Deposition

$0.00 \mathrm{E}+00$

Immersion

Inhalation

Radon

$0.00 \mathrm{E}+00$

$0.00 \mathrm{E}+00$

$0.00 \mathrm{E}+00$

$0.00 \mathrm{E}+00$

$0.00 \mathrm{E}+00$

$0.00 \mathrm{E}+00$

Ingestion

$0.00 \mathrm{E}+00$

$0.00 \mathrm{E}+00$

Source: 3

Receptor 1

Total

Source: 4

Receptor 1

Total

Source: 5

Receptor

1

Total

Source: 6

Receptor

1

Total
External

1. $90 \mathrm{E}-01$

1. $90 \mathrm{E}-01$
Deposition Immersion

$0.00 \mathrm{E}+00$

$0.00 \mathrm{E}+00$

$0.00 \mathrm{E}+00$

$0.00 \mathrm{E}+00$

Inhalation

$0.00 \mathrm{E}+00$

$0.00 \mathrm{E}+00$

Radon

$0.00 \mathrm{E}+00$

$0.00 \mathrm{E}+00$

Ingestion

$0.00 \mathrm{E}+00$

$0.00 \mathrm{E}+00$
Deposition Immersion

$0.00 \mathrm{E}+00$

$0.00 \mathrm{E}+00$

$0.00 \mathrm{E}+00$

$0.00 \mathrm{E}+00$

Inhalation

$0.00 \mathrm{E}+00$

$0.00 \mathrm{E}+00$

Radon

$0.00 \mathrm{E}+00$

$0.00 \mathrm{E}+00$

Ingestion

$0.00 \mathrm{E}+00$

$0.00 \mathrm{E}+00$
External

1. $49 \mathrm{E}+00$

1. $49 \mathrm{E}+00$
Deposition
$0.00 \mathrm{E}+00$
$0.00 \mathrm{E}+00$

Immersion
$0.00 \mathrm{E}+00$
$0.00 \mathrm{E}+00$

Inhalation

$0.00 \mathrm{E}+00$

$0.00 \mathrm{E}+00$

Radon
$0.00 \mathrm{E}+00$
$0.00 \mathrm{E}+00$
Ingestion

$0.00 \mathrm{E}+00$

$0.00 \mathrm{E}+00$

\section{External}

3. $91 \mathrm{E}-01$

3. $91 E-01$

\begin{abstract}
Deposition
$0.00 \mathrm{E}+00$
$0.00 \mathrm{E}+00$
\end{abstract}

Immersion
$0.00 \mathrm{E}+00$
$0.00 \mathrm{E}+00$

Inhalation

$0.00 \mathrm{E}+00$

$0.00 \mathrm{E}+00$

Radon

$0.00 \mathrm{E}+00$

$0.00 \mathrm{E}+00$
Ingestion

$0.00 \mathrm{E}+00$

$0.00 \mathrm{E}+00$ 
** RESRAD-BUILD Dose Program Output, Version 3.50 05/03/10 15:05:25 Page: 27 ** Title : Worst Case 1

Input File : sitel.bld

Evaluation Time: 10.0000000 years

Nuclide Detail of Doses

[mrem ]

Source: 1

$\begin{array}{lcc}\text { Nuclide } & \begin{array}{c}\text { Receptor } \\ 1\end{array} & \text { Total } \\ \text { U-235 } & 5.87 \mathrm{E}-04 & 5.87 \mathrm{E}-04 \\ \mathrm{PA}-231 & 3.70 \mathrm{E}-08 & 3.70 \mathrm{E}-08 \\ \mathrm{AC}-227 & 4.33 \mathrm{E}-08 & 4.33 \mathrm{E}-08 \\ \mathrm{CS}-137 & 2.71 \mathrm{E}-02 & 2.71 \mathrm{E}-02 \\ \text { SR-90 } & 2.73 \mathrm{E}-04 & 2.73 \mathrm{E}-04\end{array}$

Source: 2

\begin{tabular}{|c|c|c|}
\hline Nuclide & $\begin{array}{c}\text { Receptor } \\
1\end{array}$ & Total \\
\hline$U-235$ & $4.59 \mathrm{E}-04$ & $4.59 \mathrm{E}-04$ \\
\hline $\mathrm{PA}-231$ & $2.40 \mathrm{E}-08$ & $2.40 \mathrm{E}-08$ \\
\hline$A C-227$ & $3.83 E-08$ & $3.83 E-08$ \\
\hline $\mathrm{CS}-137$ & $1.92 \mathrm{E}-01$ & $1.92 \mathrm{E}-01$ \\
\hline$S R-90$ & $1.67 \mathrm{E}-03$ & $1.67 \mathrm{E}-03$ \\
\hline
\end{tabular}

Source: 3

\begin{tabular}{|c|c|c|}
\hline Nuclide & $\begin{array}{c}\text { Receptor } \\
1\end{array}$ & Total \\
\hline $\mathrm{U}-23$ & $4.54 \mathrm{E}-04$ & $4.54 \mathrm{E}-04$ \\
\hline $\mathrm{PA}$ & $2.36 \mathrm{E}-08$ & $2.36 \mathrm{E}-08$ \\
\hline$C-227$ & $3.80 E-08$ & $3.80 \mathrm{E}-08$ \\
\hline$S-137$ & $1.88 \mathrm{E}-01$ & $1.88 \mathrm{E}-01$ \\
\hline$R-90$ & $1.63 \mathrm{E}-03$ & $1.63 \mathrm{E}-03$ \\
\hline
\end{tabular}


** RESRAD-BUILD Dose Program Output, Version 3.50 05/03/10 15:05:25 Page: 28 ** Title : Worst Case 1

Input File : sitel.bld

Evaluation Time: 10.0000000 years

\section{Source: 4}

$\begin{array}{lcc}\text { Nuclide } & \begin{array}{c}\text { Receptor } \\ 1\end{array} & \text { Total } \\ & & \\ \mathrm{U}-235 & 3.47 \mathrm{E}-03 & 3.47 \mathrm{E}-03 \\ \mathrm{PA}-231 & 1.84 \mathrm{E}-07 & 1.84 \mathrm{E}-07 \\ \mathrm{AC}-227 & 2.91 \mathrm{E}-07 & 2.91 \mathrm{E}-07 \\ \mathrm{CS}-137 & 1.47 \mathrm{E}+00 & 1.47 \mathrm{E}+00 \\ \mathrm{SR}-90 & 1.28 \mathrm{E}-02 & 1.28 \mathrm{E}-02\end{array}$

Source: 5

$\begin{array}{lcc}\text { Nuclide } & \begin{array}{c}\text { Receptor } \\ 1\end{array} & \text { Total } \\ & & \\ \mathrm{U}-235 & 9.09 \mathrm{E}-04 & 9.09 \mathrm{E}-04 \\ \mathrm{PA}-231 & 4.83 \mathrm{E}-08 & 4.83 \mathrm{E}-08 \\ \mathrm{AC}-227 & 7.61 \mathrm{E}-08 & 7.61 \mathrm{E}-08 \\ \mathrm{CS}-137 & 3.86 \mathrm{E}-01 & 3.86 \mathrm{E}-01 \\ \text { SR-90 } & 3.36 \mathrm{E}-03 & 3.36 \mathrm{E}-03\end{array}$

Source: 6

Nuclide Receptor Total

1

U-235 9.09E-04 9.09E-04

$\mathrm{PA}-231 \quad 4.83 \mathrm{E}-08 \quad 4.83 \mathrm{E}-08$

$\mathrm{AC}-227 \quad 7.61 \mathrm{E}-08 \quad 7.61 \mathrm{E}-08$

$\begin{array}{lll}\mathrm{CS}-137 & 3.86 \mathrm{E}-01 & 3.86 \mathrm{E}-01\end{array}$

SR-90 3.36E-03 3.36E-03 
** RESRAD-BUILD Dose Program Output, Version 3.50 05/03/10 15:05:25 Page: 29 ** Title : Worst Case 1

Input File : sitel.bld

Evaluation Time: 30.0000000 years

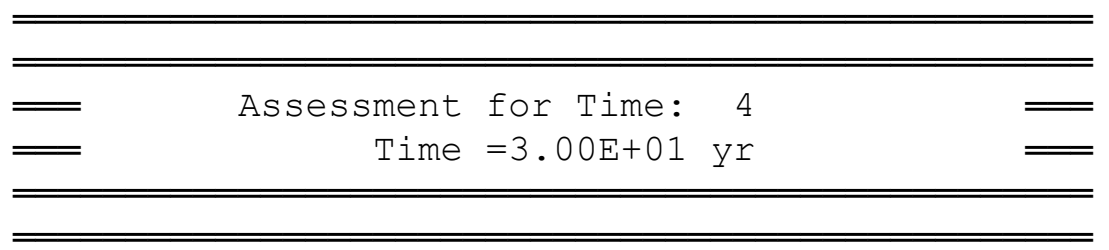

$=$ Source Information $=$

Source: 1

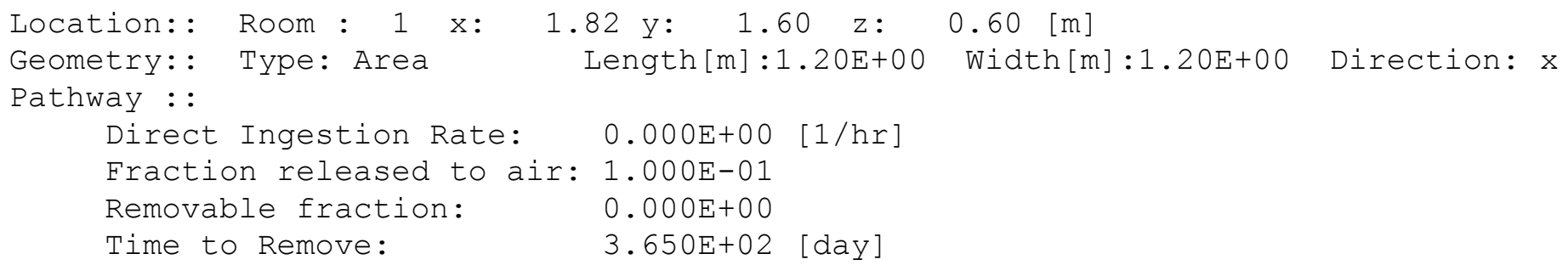

Contamination: : Nuclide Concentration

$[\mathrm{dpm} / \mathrm{m} 2]$

$\mathrm{U}-235 \quad 7.200 \mathrm{E}+03$

$\mathrm{PA}-231 \quad 4.563 \mathrm{E}+00$

$\mathrm{AC}-227 \quad 1.622 \mathrm{E}+00$

$\mathrm{CS}-137 \quad 6.750 \mathrm{E}+04$

SR-90 $\quad 6.607 \mathrm{E}+04$

Source: 2

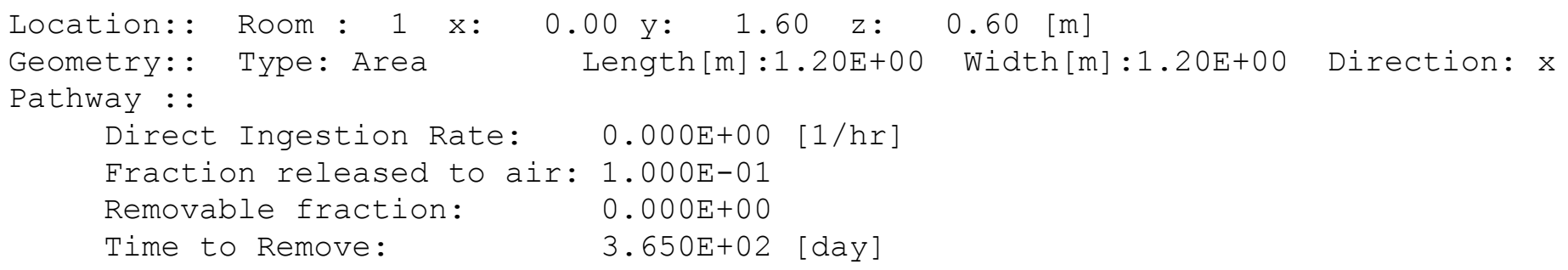


* RESRAD-BUILD Dose Program Output, Version 3.50 05/03/10 15:05:25 Page: 30 ** Title : Worst Case 1

Input File : sitel.bld

Evaluation Time: 30.0000000 years

\section{Source: 3}

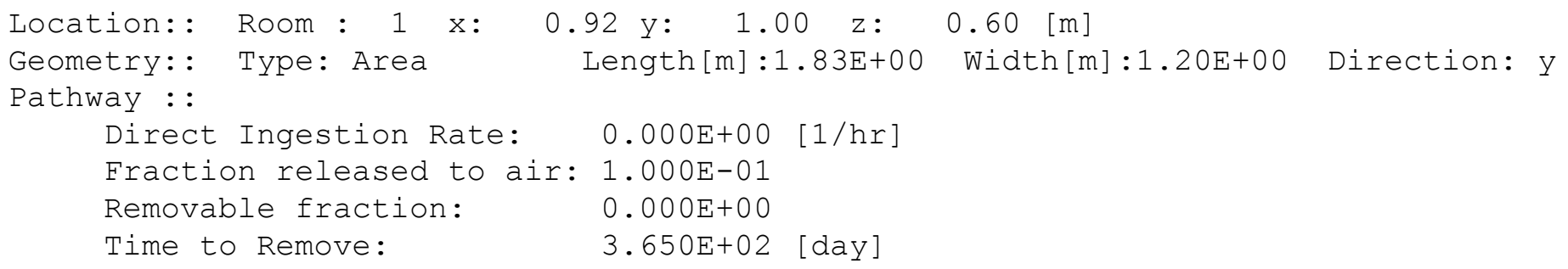

\begin{tabular}{|c|c|c|}
\hline \multirow[t]{6}{*}{ Contamination: : } & Nuclide & $\begin{array}{c}\text { Concentration } \\
{[\mathrm{dpm} / \mathrm{m} 2]}\end{array}$ \\
\hline & $\mathrm{U}-235$ & $7.200 E+03$ \\
\hline & $\mathrm{PA}-231$ & $4.563 E+00$ \\
\hline & $A C-227$ & $1.622 \mathrm{E}+00$ \\
\hline & CS-137 & $4.950 E+05$ \\
\hline & $S R-90$ & $4.845 E+05$ \\
\hline
\end{tabular}

Source: 4

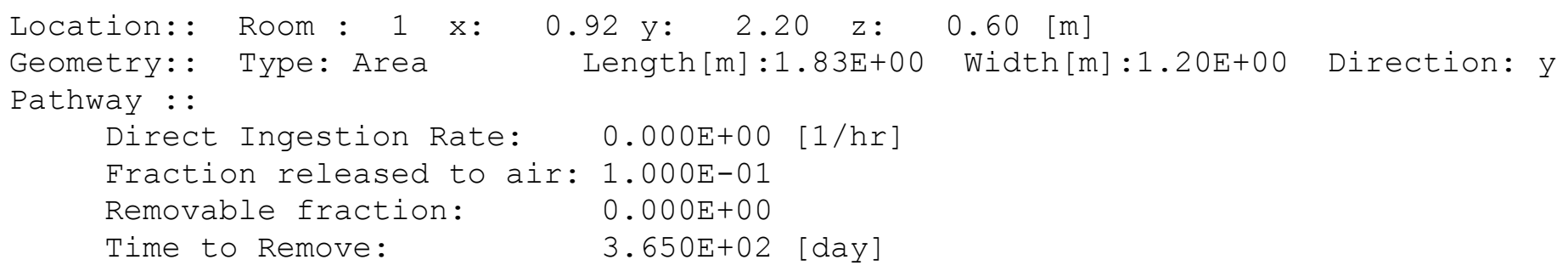


** RESRAD-BUILD Dose Program Output, Version 3.50 05/03/10 15:05:25 Page: 31 ** Title : Worst Case 1

Input File : sitel.bld

Evaluation Time: 30.0000000 years

Source: 5

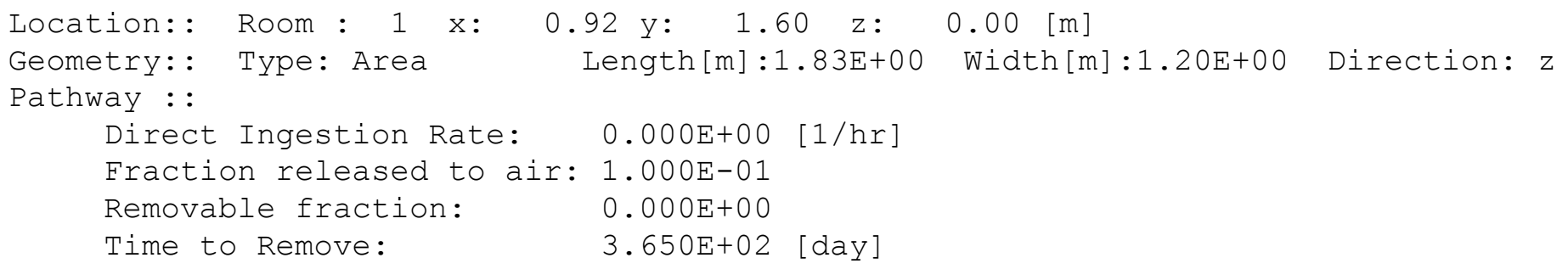

\begin{tabular}{|c|c|c|}
\hline \multirow[t]{6}{*}{ Contamination: : } & Nuclide & $\begin{array}{c}\text { Concentration } \\
{[\mathrm{dpm} / \mathrm{m} 2]}\end{array}$ \\
\hline & $U-235$ & $7.200 \mathrm{E}+03$ \\
\hline & $P A-231$ & $4.563 E+00$ \\
\hline & $A C-227$ & $1.622 \mathrm{E}+00$ \\
\hline & CS -137 & $4.950 \mathrm{E}+05$ \\
\hline & $S R-90$ & $4.845 E+05$ \\
\hline
\end{tabular}

Source: 6

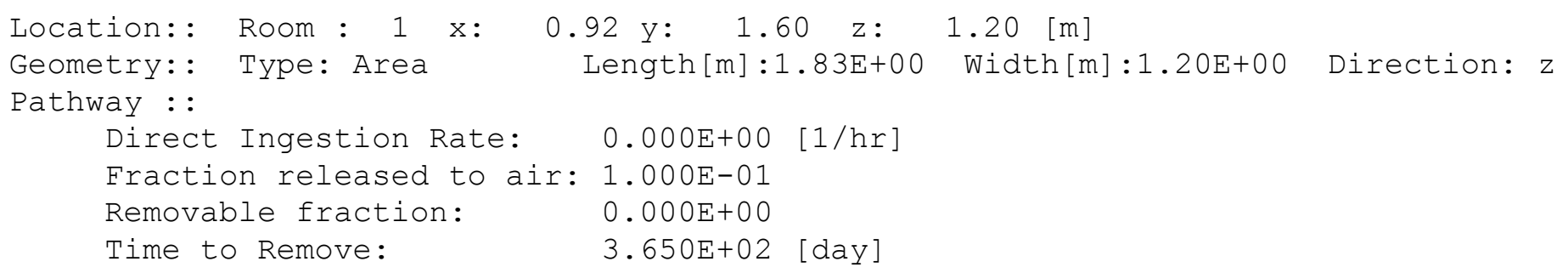


** RESRAD-BUILD Dose Program Output, Version 3.50 05/03/10 15:05:25 Page: 32 ** Title : Worst Case 1

Input File : sitel.bld

Evaluation Time: 30.0000000 years

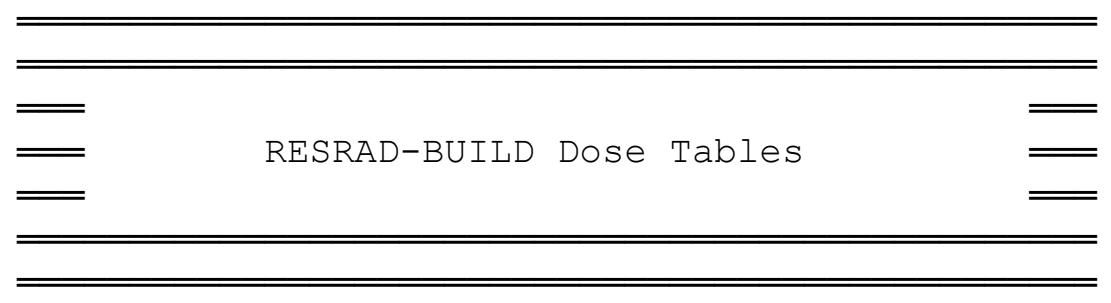

Source Contributions to Receptor Doses

=

[mrem]

Source Source Source Source source source Total

$\begin{array}{lllllll}1 & 2 & 3 & 4 & 5\end{array}$

Receptor $1 \quad 1.79 \mathrm{E}-02 \quad 1.22 \mathrm{E}-01 \quad 1.20 \mathrm{E}-01 \quad 9.39 \mathrm{E}-01 \quad 2.46 \mathrm{E}-01 \quad 2.46 \mathrm{E}-01 \quad 1.69 \mathrm{E}+00$

$\begin{array}{lllllllll}1.79 \mathrm{E}-02 & 1.22 \mathrm{E}-01 & 1.20 \mathrm{E}-01 & 9.39 \mathrm{E}-01 & 2.46 \mathrm{E}-01 & 2.46 \mathrm{E}-01 & 1.69 \mathrm{E}+00\end{array}$ 
** RESRAD-BUILD Dose Program Output, Version 3.50 05/03/10 15:05:25 Page: 33 ** Title : Worst Case 1

Input File : sitel.bld

Evaluation Time: 30.0000000 years

Pathway Detail of Doses

Source: 1

Receptor External

$1 \quad 1.79 \mathrm{E}-02$

Total

$1.79 \mathrm{E}-02$

\section{[mrem $]$}

Deposition Immersion

Inhalation

Radon

$0.00 \mathrm{E}+00$

$0.00 \mathrm{E}+00$

Ingestion

$0.00 \mathrm{E}+00$

$0.00 \mathrm{E}+00$

$0.00 \mathrm{E}+00$

$0.00 \mathrm{E}+00$

$0.00 \mathrm{E}+00$

$0.00 \mathrm{E}+00$
Source: 2

Receptor 1

Total

Source: 3

Receptor 1

Total

Source: 4

Receptor 1

Total

Source: 5

Receptor

1

Total

Source: 6

Receptor 1

Total
External

1.22E-01

$1.22 \mathrm{E}-01$
Deposition Immersion

$0.00 \mathrm{E}+00$

$0.00 \mathrm{E}+00$

$0.00 \mathrm{E}+00$

$0.00 \mathrm{E}+00$

Inhalation

$0.00 \mathrm{E}+00$

$0.00 \mathrm{E}+00$

Radon

$0.00 \mathrm{E}+00$

$0.00 \mathrm{E}+00$

Ingestion

$0.00 \mathrm{E}+00$

$0.00 \mathrm{E}+00$
External

$1.20 \mathrm{E}-01$

$1.20 \mathrm{E}-01$
Deposition Immersion

$0.00 \mathrm{E}+00$

$0.00 \mathrm{E}+00$

$0.00 \mathrm{E}+00$

$0.00 \mathrm{E}+00$

Inhalation

$0.00 \mathrm{E}+00$

$0.00 \mathrm{E}+00$

Radon

$0.00 \mathrm{E}+00$

$0.00 \mathrm{E}+00$

Ingestion

$0.00 \mathrm{E}+00$

$0.00 \mathrm{E}+00$
External

9.39E-01

$9.39 \mathrm{E}-01$
Deposition
$0.00 \mathrm{E}+00$
$0.00 \mathrm{E}+00$
Inhalation Radon

$0.00 \mathrm{E}+00$

$0.00 \mathrm{E}+00$

$0.00 \mathrm{E}+00$

$0.00 \mathrm{E}+00$
Ingestion

$0.00 \mathrm{E}+00$

$0.00 \mathrm{E}+00$
External

2. $46 \mathrm{E}-01$

$2.46 \mathrm{E}-01$
Deposition
$0.00 \mathrm{E}+00$
$0.00 \mathrm{E}+00$

Immersion

Inhalation

$0.00 \mathrm{E}+00$

$0.00 \mathrm{E}+00$
Radon

$0.00 \mathrm{E}+00$

$0.00 \mathrm{E}+00$
Ingestion

$0.00 \mathrm{E}+00$

$0.00 \mathrm{E}+00$
External

2. $46 \mathrm{E}-01$

2. $46 \mathrm{E}-01$ $\begin{array}{ll}\text { Deposition } & \text { Immersion } \\ 0.00 \mathrm{E}+00 & 0.00 \mathrm{E}+00 \\ 0.00 \mathrm{E}+00 & 0.00 \mathrm{E}+00\end{array}$

Inhalation Radon

$0.00 \mathrm{E}+00 \quad 0.00 \mathrm{E}+00$

$0.00 \mathrm{E}+00 \quad 0.00 \mathrm{E}+00$
Ingestion

$0.00 \mathrm{E}+00$

$0.00 \mathrm{E}+00$ 
** RESRAD-BUILD Dose Program Output, Version 3.50 05/03/10 15:05:25 Page: 34 ** Title : Worst Case 1

Input File : sitel.bld

Evaluation Time: 30.0000000 years

Nuclide Detail of Doses

[mrem ]

Source: 1

$\begin{array}{lcc}\text { Nuclide } & \begin{array}{c}\text { Receptor } \\ 1\end{array} & \text { Total } \\ & & \\ \mathrm{U}-235 & 5.87 \mathrm{E}-04 & 5.87 \mathrm{E}-04 \\ \mathrm{PA}-231 & 1.07 \mathrm{E}-07 & 1.07 \mathrm{E}-07 \\ \mathrm{AC}-227 & 3.02 \mathrm{E}-07 & 3.02 \mathrm{E}-07 \\ \mathrm{CS}-137 & 1.71 \mathrm{E}-02 & 1.71 \mathrm{E}-02 \\ \text { SR-90 } & 1.70 \mathrm{E}-04 & 1.70 \mathrm{E}-04\end{array}$

Source: 2

$\begin{array}{lcc}\text { Nuclide } & \begin{array}{c}\text { Receptor } \\ 1\end{array} & \text { Total } \\ & & \\ \mathrm{U}-235 & 4.59 \mathrm{E}-04 & 4.59 \mathrm{E}-04 \\ \mathrm{PA}-231 & 6.98 \mathrm{E}-08 & 6.98 \mathrm{E}-08 \\ \mathrm{AC}-227 & 2.67 \mathrm{E}-07 & 2.67 \mathrm{E}-07 \\ \mathrm{CS}-137 & 1.21 \mathrm{E}-01 & 1.21 \mathrm{E}-01 \\ \text { SR-90 } & 1.04 \mathrm{E}-03 & 1.04 \mathrm{E}-03\end{array}$

Source: 3

$\begin{array}{lcc}\text { Nuclide } & \begin{array}{c}\text { Receptor } \\ 1\end{array} & \text { Total } \\ & & \\ \mathrm{U}-235 & 4.54 \mathrm{E}-04 & 4.54 \mathrm{E}-04 \\ \mathrm{PA}-231 & 6.84 \mathrm{E}-08 & 6.84 \mathrm{E}-08 \\ \mathrm{AC}-227 & 2.65 \mathrm{E}-07 & 2.65 \mathrm{E}-07 \\ \mathrm{CS}-137 & 1.18 \mathrm{E}-01 & 1.18 \mathrm{E}-01 \\ \text { SR-90 } & 1.01 \mathrm{E}-03 & 1.01 \mathrm{E}-03\end{array}$


** RESRAD-BUILD Dose Program Output, Version 3.50 05/03/10 15:05:25 Page: 35 ** Title : Worst Case 1

Input File : sitel.bld

Evaluation Time: 30.0000000 years

\section{Source: 4}

$\begin{array}{lcc}\text { Nuclide } & \begin{array}{c}\text { Receptor } \\ 1\end{array} & \text { Total } \\ \text { U-235 } & 3.47 \mathrm{E}-03 & 3.47 \mathrm{E}-03 \\ \mathrm{PA}-231 & 5.35 \mathrm{E}-07 & 5.35 \mathrm{E}-07 \\ \mathrm{AC}-227 & 2.03 \mathrm{E}-06 & 2.03 \mathrm{E}-06 \\ \mathrm{CS}-137 & 9.28 \mathrm{E}-01 & 9.28 \mathrm{E}-01 \\ \text { SR-90 } & 7.96 \mathrm{E}-03 & 7.96 \mathrm{E}-03\end{array}$

Source: 5

$\begin{array}{lcc}\text { Nuclide } & \begin{array}{c}\text { Receptor } \\ 1\end{array} & \text { Total } \\ & \begin{array}{l}\text { T-09E-04 } \\ \text { U-235 }\end{array} & 9.09 \mathrm{E}-04 \\ \mathrm{PA}-231 & 1.40 \mathrm{E}-07 & 1.40 \mathrm{E}-07 \\ \mathrm{AC}-227 & 5.30 \mathrm{E}-07 & 5.30 \mathrm{E}-07 \\ \mathrm{CS}-137 & 2.43 \mathrm{E}-01 & 2.43 \mathrm{E}-01 \\ \mathrm{SR}-90 & 2.09 \mathrm{E}-03 & 2.09 \mathrm{E}-03\end{array}$

Source: 6

Nuclide Receptor Total

1

$\mathrm{U}-2359.09 \mathrm{E}-04 \quad 9.09 \mathrm{E}-04$

PA-231 1.40E-07 1.40E-07

$\begin{array}{lll}\mathrm{AC}-227 & 5.30 \mathrm{E}-07 & 5.30 \mathrm{E}-07\end{array}$

$\begin{array}{lll}C S-137 & 2.43 \mathrm{E}-01 & 2.43 \mathrm{E}-01\end{array}$

SR-90 2.09E-03 2.09E-03 
** RESRAD-BUILD Dose Program Output, Version 3.50 05/03/10 15:05:25 Page: 36 ** Title : Worst Case 1

Input File : sitel.bld

Full Summary

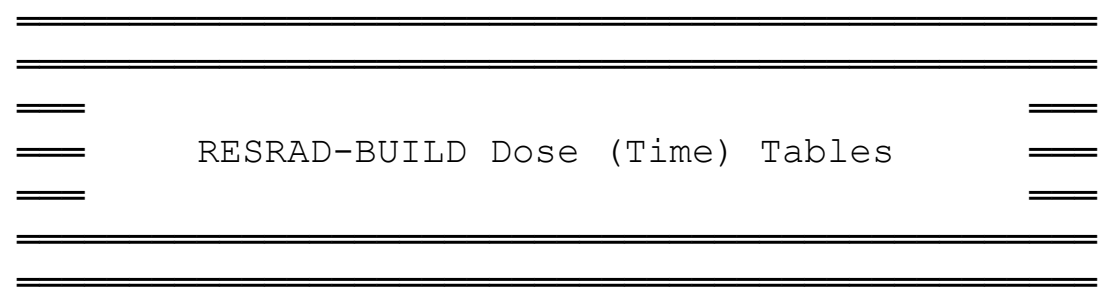

Receptor Dose Received for the Exposure Duration

(mrem)

Evaluation Time [yr]

$0.00 \mathrm{E}+00 \quad 1.00 \mathrm{E}+00 \quad 1.00 \mathrm{E}+01 \quad 3.00 \mathrm{E}+01$

$\begin{array}{lllll}1 & 3.83 \mathrm{E}+00 & 3.30 \mathrm{E}+00 & 2.68 \mathrm{E}+00 & 1.69 \mathrm{E}+00\end{array}$

Receptor Dose/Yr Averaged Over Exposure Duration

ב

(mrem/yr)

Evaluation Time [yr]

$0.00 \mathrm{E}+00 \quad 1.00 \mathrm{E}+00 \quad 1.00 \mathrm{E}+01 \quad 3.00 \mathrm{E}+01$

$\begin{array}{lllll}13.83 \mathrm{E}+00 & 3.30 \mathrm{E}+00 & 2.68 \mathrm{E}+00 & 1.69 \mathrm{E}+00\end{array}$ 
Appendix E

Results from RESRAD-BUILD

Worst Case Scenario 2 
* RESRAD-BUILD Dose Program Output, Version 3.50 05/04/10 09:36:49 Page: 1 ** Title : Worst Case 2 - Grind

Input File : sitel.bld

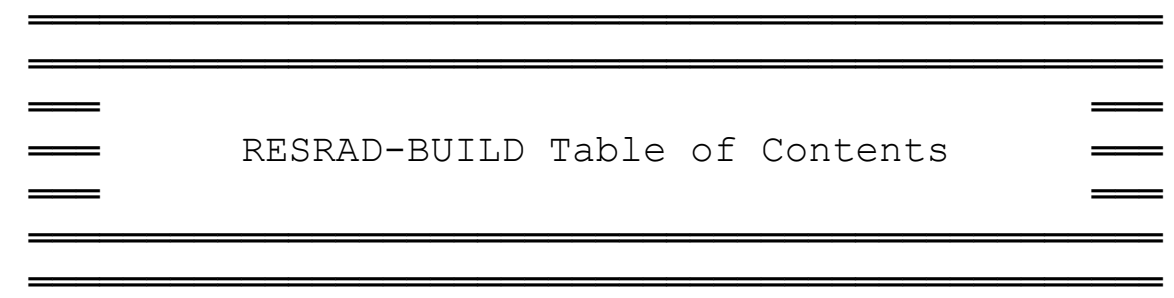

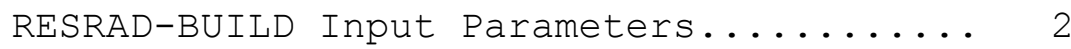

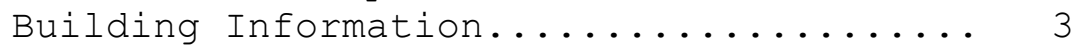

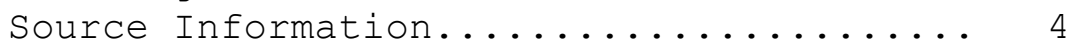

For time $=0.00 \mathrm{E}+00 \mathrm{yr}$

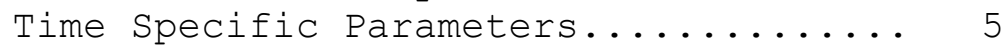

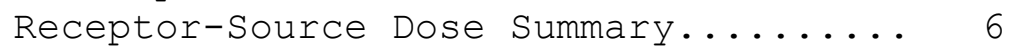

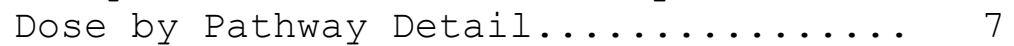

Dose by Nuclide Detail............ 8

For time $=1.00 \mathrm{E}+00 \mathrm{yr}$

Time Specific Parameters............ 9

For time $=1.00 \mathrm{E}+01 \mathrm{yr}$

Time Specific Parameters........... 10

For time $=3.00 \mathrm{E}+01 \mathrm{yr}$

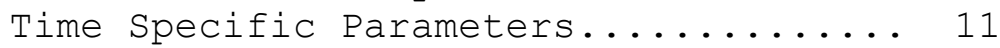

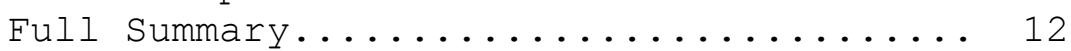


* RESRAD-BUILD Dose Program Output, Version 3.50 05/04/10 09:36:49 Page: 2 ** Title : Worst Case 2 - Grind

Input File : sitel.bld

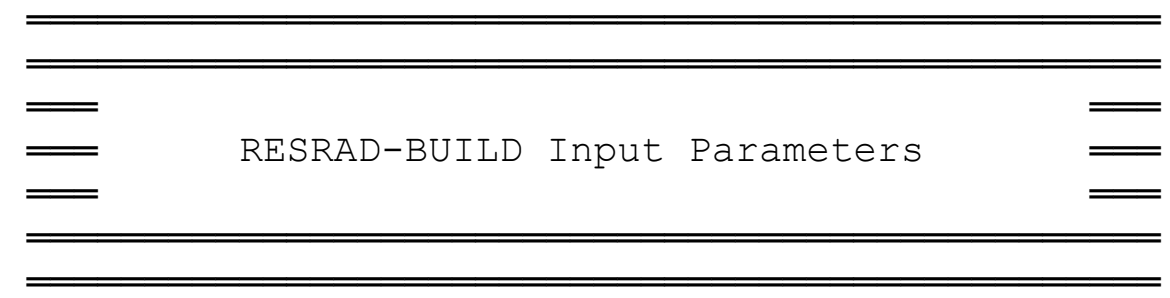

Number of Sources : 1

Number of Receptors: 1

Total Time : $1.000000 \mathrm{E}+00$ days

Fraction Inside : $5.000000 \mathrm{E}-01$

$\overline{=}$ Receptor Information $=$

Receptor

Room

x $\quad y$

$[\mathrm{m}] \quad[\mathrm{m}]$

11.000

$y$
$[\mathrm{~m}]$
1.000

z Fractime

Inhalation

[m]

$1.000 \quad 0.500$

[m3/day]

1. $80 \mathrm{E}+01$

Ingestion (Dust)

[m2/hr]

1. $00 \mathrm{E}-04$

\begin{tabular}{|c|c|c|c|c|}
\hline Receptor & Source & $\begin{array}{l}\text { Density } \\
{[\mathrm{g} / \mathrm{cm} 3]}\end{array}$ & $\begin{array}{l}\text { Thickness } \\
{[\mathrm{cm}]}\end{array}$ & Material \\
\hline 1 & 1 & $2.40 \mathrm{E}+00$ & $0.00 \mathrm{E}+00$ & co \\
\hline
\end{tabular}


* RESRAD-BUILD Dose Program Output, Version 3.50 05/04/10 09:36:49 Page: 3 ** Title : Worst Case 2 - Grind

Input File : sitel.bld

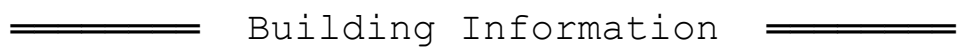

Building Air Exchange Rate: 8.00E-01 1/hr

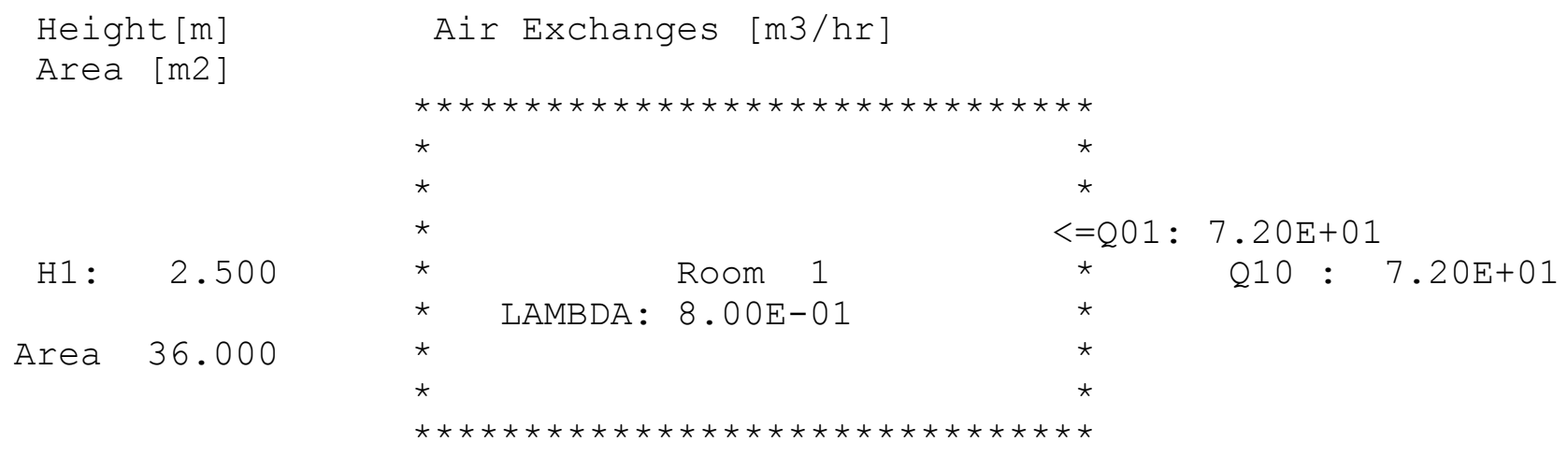

Deposition velocity: 1.00E-02 [m/s] Resuspension Rate: 5.00E-07 [1/s] 
** RESRAD-BUILD Dose Program Output, Version 3.50 05/04/10 09:36:49 Page: 4 ** Title : Worst Case 2 - Grind

Input File : sitel.bld

$\overline{=}$ Source Information $=$

Source: 1

Location: : Room : $1 \mathrm{x}: 1.30 \mathrm{y}: 1.00 \mathrm{z}: 1.00[\mathrm{~m}]$

Geometry:: Type: Area Length $[\mathrm{m}]: 1.00 \mathrm{E}+00$ Width $[\mathrm{m}]: 1.00 \mathrm{E}+00$ Direction: $x$

Pathway : :

Direct Ingestion Rate: 1.000E-01 [1/hr]

Fraction released to air: 5.000E-01

Removable fraction: $\quad 1.000 \mathrm{E}+00$

Time to Remove: 2.500E-01 [day]

Contamination: :

Nuclide Concentration

Dose Conversion Factor (Library: ICRP 72 (Adult))

\begin{tabular}{llllll}
\cline { 5 - 6 } \cline { 5 - 6 } & {$[\mathrm{dpm} / \mathrm{m} 2]$} & & $\begin{array}{c}\text { Ingestion } \\
{[\mathrm{mrem} / \mathrm{dpm}]}\end{array}$ & $\begin{array}{c}\text { Inhalation } \\
{[\mathrm{mrem} / \mathrm{dpm}]}\end{array}$ & $\begin{array}{c}\text { Submersion } \\
{[\mathrm{mrem} / \mathrm{yr} /} \\
(\mathrm{dpm} / \mathrm{m} 3)]\end{array}$ \\
$\mathrm{U}-235$ & $8.000 \mathrm{E}+03$ & $7.890 \mathrm{E}-05$ & $1.417 \mathrm{E}-02$ & $4.063 \mathrm{E}-04$ \\
$\mathrm{PA}-231$ & $0.000 \mathrm{E}+00$ & $1.183 \mathrm{E}-03$ & $2.333 \mathrm{E}-01$ & $9.049 \mathrm{E}-05$ \\
$\mathrm{AC}-227$ & $0.000 \mathrm{E}+00$ & $2.015 \mathrm{E}-03$ & $9.476 \mathrm{E}-01$ & $9.734 \mathrm{E}-04$ \\
$\mathrm{CS}-137$ & $1.500 \mathrm{E}+05$ & $2.167 \mathrm{E}-05$ & $6.500 \mathrm{E}-05$ & $1.434 \mathrm{E}-03$ \\
$\mathrm{SR}-90$ & $1.500 \mathrm{E}+05$ & $5.117 \mathrm{E}-05$ & $2.692 \mathrm{E}-04$ & $1.039 \mathrm{E}-05$
\end{tabular}


** RESRAD-BUILD Dose Program Output, Version 3.50 05/04/10 09:36:49 Page: 5 ** Title : Worst Case 2 - Grind

Input File : sitel.bld

Evaluation Time: $0.00000000 \mathrm{E}+00$ years

\begin{tabular}{rrrrr}
\hline \hline & & & & \\
\hline \hline & Assessment for Time: & 1 & $=$ \\
\hline \hline & Time & $=0.00 \mathrm{E}+00$ & yr & \\
\hline \hline
\end{tabular}

$=$ Source Information

Source: 1

Location: : Room : $1 \mathrm{x}: 1.30 \mathrm{y}: 1.00 \mathrm{z}: 1.00$ [m]

Geometry:: Type: Area Length $[\mathrm{m}]: 1.00 \mathrm{E}+00$ Width $[\mathrm{m}]: 1.00 \mathrm{E}+00$ Direction: $\mathrm{x}$

Pathway : :

Direct Ingestion Rate: $1.000 \mathrm{E}-01$ [1/hr]

Fraction released to air: 5.000E-01

Removable fraction: $\quad 1.000 \mathrm{E}+00$

Time to Remove: 2.500E-01 [day]

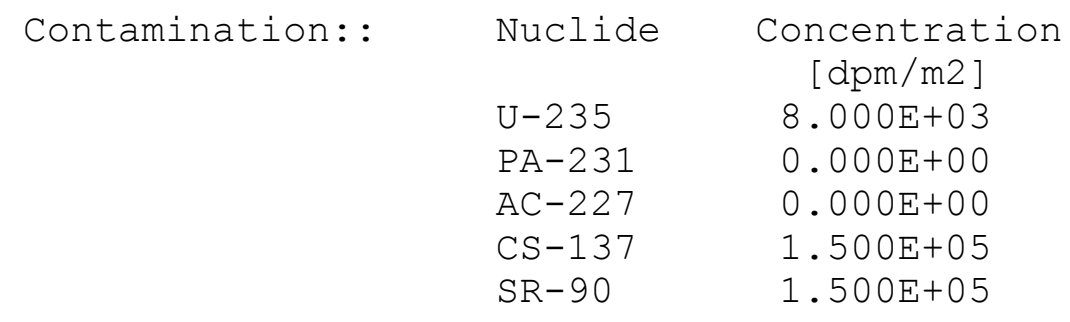


* RESRAD-BUILD Dose Program Output, Version 3.50 05/04/10 09:36:49 Page: 6 ** Title : Worst Case 2 - Grind

Input File : sitel.bld

Evaluation Time: $0.00000000 \mathrm{E}+00$ years

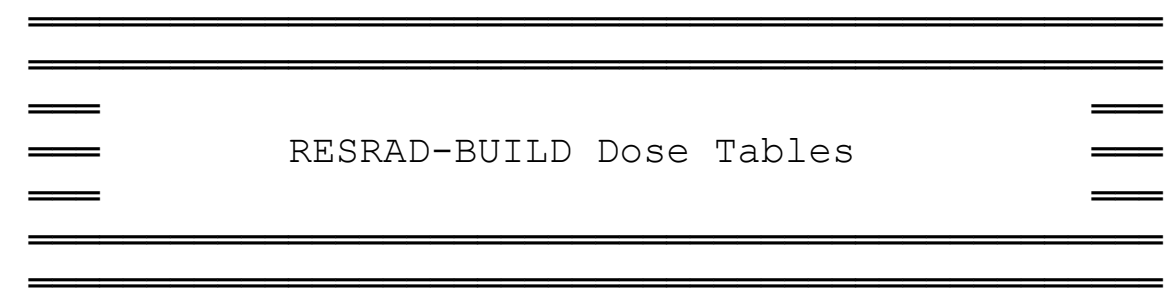

Source Contributions to Receptor Doses

=

[mrem]

\section{Source Total}

1

Receptor $1 \quad 1.16 \mathrm{E}+001.16 \mathrm{E}+00$

Total $1.16 \mathrm{E}+001.16 \mathrm{E}+00$ 
* RESRAD-BUILD Dose Program Output, Version 3.50 05/04/10 09:36:49 Page: 7 ** Title : Worst Case 2 - Grind

Input File : sitel.bld

Evaluation Time: $0.00000000 \mathrm{E}+00$ years

Pathway Detail of Doses

[mrem ]
Source: 1

Receptor 1

Total
External 5. 49E-05

5. 49E-05
Deposition 1.77E-02

1. $77 \mathrm{E}-02$
Immersion

4. 61E-05

4. $61 \mathrm{E}-05$
Inhalation

2. 29E-01

2. 29E-01
Radon $0.00 \mathrm{E}+00$

$0.00 \mathrm{E}+00$
Ingestion

9.09E-01

9.09E-01 
* RESRAD-BUILD Dose Program Output, Version 3.50 05/04/10 09:36:49 Page: 8 ** Title : Worst Case 2 - Grind

Input File : sitel.bld

Evaluation Time: $0.00000000 \mathrm{E}+00$ years

Nuclide Detail of Doses

[mrem ]

Source: 1

$\begin{array}{lcc}\text { Nuclide } & \begin{array}{c}\text { Receptor } \\ 1\end{array} & \text { Total } \\ \text { U-235 } & 2.10 \mathrm{E}-01 & 2.10 \mathrm{E}-01 \\ \mathrm{PA}-231 & 3.23 \mathrm{E}-08 & 3.23 \mathrm{E}-08 \\ \mathrm{AC}-227 & 1.17 \mathrm{E}-12 & 1.17 \mathrm{E}-12 \\ \mathrm{CS}-137 & 2.86 \mathrm{E}-01 & 2.86 \mathrm{E}-01 \\ \mathrm{SR}-90 & 6.59 \mathrm{E}-01 & 6.59 \mathrm{E}-01\end{array}$


** RESRAD-BUILD Dose Program Output, Version 3.50 05/04/10 09:36:49 Page: 9 ** Title : Worst Case 2 - Grind

Input File : sitel.bld

Evaluation Time: 1.00000000 years

\begin{tabular}{rrrrr}
\hline \hline & & & & \\
\hline \hline & Assessment for Time: & 2 & $=$ \\
\hline \hline & Time & $=1.00 \mathrm{E}+00$ & yr & \\
\hline \hline
\end{tabular}

$\overline{=}$ Source Information $=$

Source: 1

Location: : Room : $1 \mathrm{x}: 1.30 \mathrm{y}: 1.00 \mathrm{z}: 1.00$ [m]

Geometry:: Type: Area Length $[\mathrm{m}]: 1.00 \mathrm{E}+00$ Width $[\mathrm{m}]: 1.00 \mathrm{E}+00$ Direction: $\mathrm{x}$

Pathway : :

Direct Ingestion Rate: $1.000 \mathrm{E}-01$ [1/hr]

Fraction released to air: 5.000E-01

Removable fraction: $\quad 0.000 \mathrm{E}+00$

Time to Remove: 2.500E-01 [day]

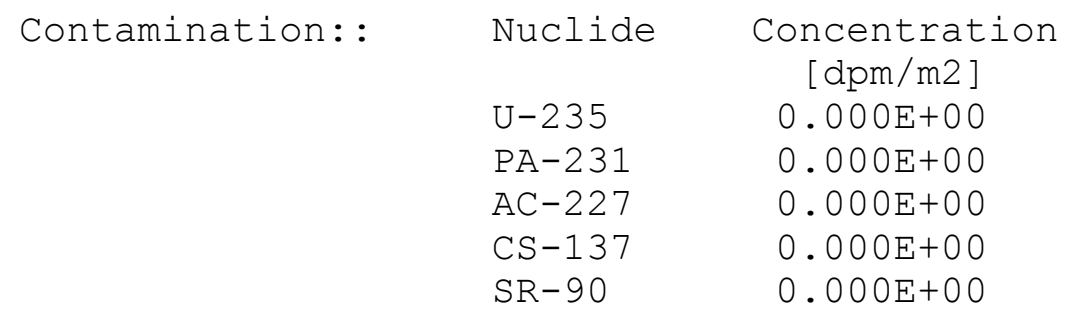


* RESRAD-BUILD Dose Program Output, Version 3.50 05/04/10 09:36:49 Page: 10 ** Title : Worst Case 2 - Grind

Input File : sitel.bld

Evaluation Time: 10.0000000 years

\begin{tabular}{rrrrr}
\hline \hline & & & & \\
\hline \hline & Assessment for Time: & 3 & $=$ \\
\hline \hline & Time & $=1.00 \mathrm{E}+01$ & yr & \\
\hline \hline
\end{tabular}

$=$ Source Information

Source: 1

Location: : Room : $1 \mathrm{x}: 1.30 \mathrm{y}: 1.00 \mathrm{z}: 1.00$ [m]

Geometry:: Type: Area Length $[\mathrm{m}]: 1.00 \mathrm{E}+00$ Width $[\mathrm{m}]: 1.00 \mathrm{E}+00$ Direction: $\mathrm{x}$

Pathway : :

Direct Ingestion Rate: $1.000 \mathrm{E}-01$ [1/hr]

Fraction released to air: 5.000E-01

Removable fraction: $\quad 0.000 \mathrm{E}+00$

Time to Remove: 2.500E-01 [day]

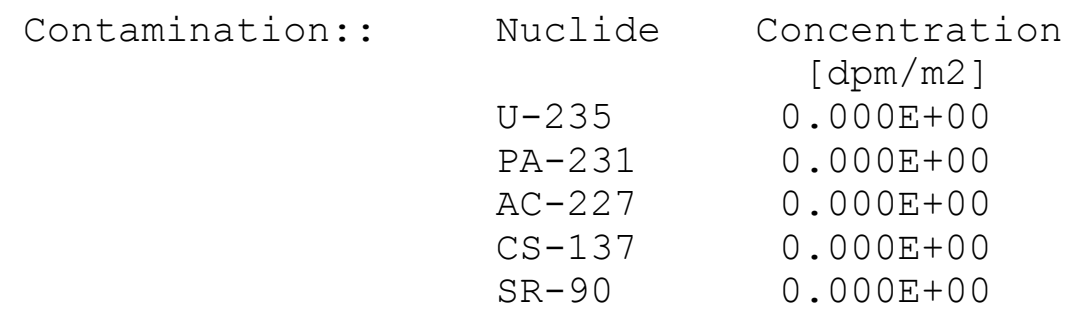


** RESRAD-BUILD Dose Program Output, Version 3.50 05/04/10 09:36:49 Page: 11 ** Title : Worst Case 2 - Grind

Input File : sitel.bld

Evaluation Time: 30.0000000 years

\begin{tabular}{rrrrr}
\hline \hline & & & & \\
\hline \hline & Assessment for Time: & 4 & $=$ \\
\hline \hline & Time & $=3.00 \mathrm{E}+01$ & yr & \\
\hline \hline
\end{tabular}

$=$ Source Information

Source: 1

Location: : Room : $1 \mathrm{x}: 1.30 \mathrm{y}: 1.00 \mathrm{z}: 1.00$ [m]

Geometry:: Type: Area Length $[\mathrm{m}]: 1.00 \mathrm{E}+00$ Width $[\mathrm{m}]: 1.00 \mathrm{E}+00$ Direction: $\mathrm{x}$

Pathway : :

Direct Ingestion Rate: $1.000 \mathrm{E}-01$ [1/hr]

Fraction released to air: 5.000E-01

Removable fraction: $\quad 0.000 \mathrm{E}+00$

Time to Remove: 2.500E-01 [day]

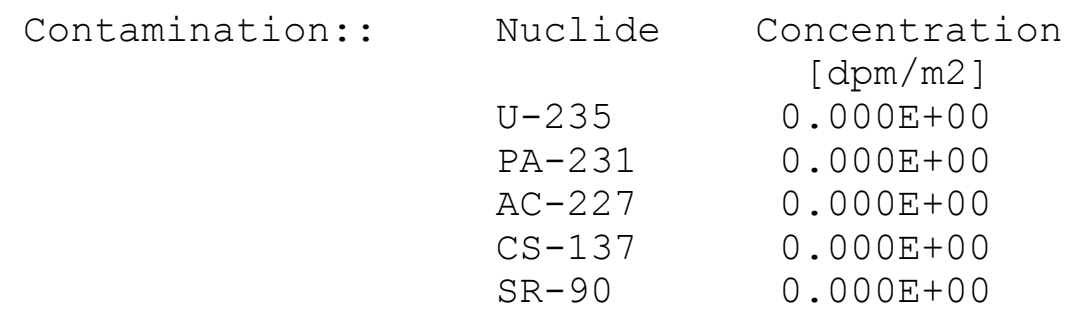


* RESRAD-BUILD Dose Program Output, Version 3.50 05/04/10 09:36:49 Page: 12 ** Title : Worst Case 2 - Grind

Input File : sitel.bld

Full Summary

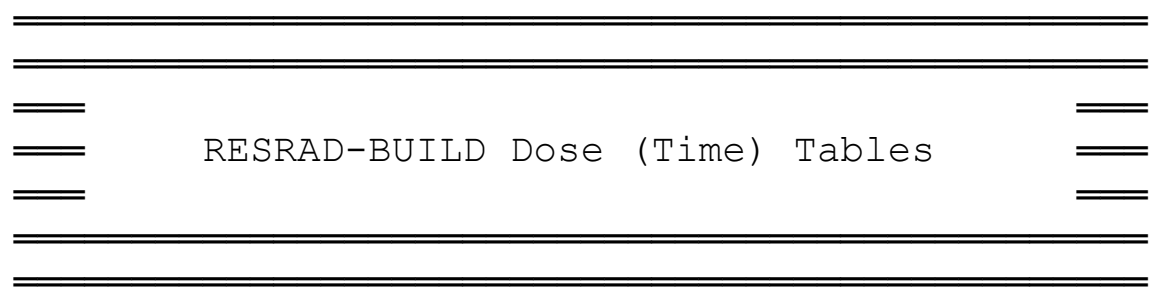

Receptor Dose Received for the Exposure Duration

=

(mrem)

Evaluation Time [yr]

$0.00 \mathrm{E}+00 \quad 1.00 \mathrm{E}+00 \quad 1.00 \mathrm{E}+01 \quad 3.00 \mathrm{E}+01$

$1 \quad 1.16 \mathrm{E}+00 \quad 0.00 \mathrm{E}+00 \quad 0.00 \mathrm{E}+00 \quad 0.00 \mathrm{E}+00$

Receptor Dose/Yr Averaged Over Exposure Duration

(mrem/yr)

Evaluation Time [yr]

$0.00 \mathrm{E}+00 \quad 1.00 \mathrm{E}+00 \quad 1.00 \mathrm{E}+01 \quad 3.00 \mathrm{E}+01$

$14.22 \mathrm{E}+02 \quad 0.00 \mathrm{E}+00 \quad 0.00 \mathrm{E}+00 \quad 0.00 \mathrm{E}+00$ 